\title{
Lifting the School Mat: An Investigation of Pedagogy and Children's Social Worlds at Mat Time
}

\author{
Anita Dellahay Mortlock \\ A thesis submitted to the Victoria University of Wellington in fulfillment of the \\ requirements for the degree of Doctor of Philosophy \\ Victoria University of Wellington \\ New Zealand
}

2016 
Dedicated to my four Grandparents: Charles and Anne Mortlock, Alan and Della Boshier. 


\begin{abstract}
Primary school teachers' use of whole-class activity is a well-documented phenomenon. Typically, it is assumed that children's active participation in group tasks is important for their academic learning and for developing self-concept. However, previous studies have found that children's participation varies widely. The present research set out to investigate why individual children's participation differs within the peer group during whole-class activity. Teachers' pedagogy and children's social worlds intersect during classroom interactions; it is in this intersecting space that this research is situated.
\end{abstract}

One specific whole-class activity was chosen as a focus, namely mat time. This is a practice whereby the teacher calls the entire class to the mat typically for the purposes of instruction, discussion, or other similar activity. To understand mat time from the perspectives of the people who experience it, two studies were undertaken using a mixed strategy approach for data gathering. The first study investigated teachers' perspectives $(\mathrm{N}=296)$ using a questionnaire. Participants were asked about a variety of themes relating to mat time including pedagogical uses, strategies, and outcomes. Principal components analyses confirmed the approximate uni-dimensionality of the data relating to each theme, which were then calibrated to a measurement variable using Samejima's (1969, as cited in DeMars, 2010) graded response model. Various correlations and comparisons were conducted pertaining to the pedagogical factors influencing children's participation, behaviour, and enjoyment. The second study used qualitative semistructured interviews with children $(n=49)$ from three year two classrooms situated in different schools. The data were analysed and discussed in relation to peer culture and peer-relations theories, which posit that children's social groups consist of norms and interests that differ to those of adults', and that such groups consist of internal social hierarchies. 
Taken together, the findings from the two studies indicated that teachers and children differ in their perception of the social climate at mat time. For instance, whereas teachers tended to report that mat time achieved prosocial objectives, children were more likely to describe socially divisive aspects. Such aspects included certain children's desire to affiliate with specific peers while excluding others, or promoting their own participation over that of classmates. Seating position and opportunities to take active roles were sources of competition. Children's differing participation was influenced by their individual strategic understandings of how to secure active roles, social support, and academic confidence. Furthermore, teachers generally reported that children were inattentive during mat time, suggesting that it may be an ineffective context for learning. Nevertheless, when teachers were cognisant of children's interests, they tended to report better participation across the class. The implications for teaching practice include an onus for teachers to actively protect vulnerable children during mat time, socially and academically, and to ensure that opportunities to take part in activities are equitably distributed. 


\section{ACKNOWLEDGMENTS}

May we rediscover the power of circles, of sitting in council to listen and learn; the power of trusting the wisdom that emerges from the voices that are quietest, least valued, or that we least expect to learn from. May we recall the power of listening patiently for the intelligence of the whole to emerge, without rushing toward conclusions.

-Nina Simons

I am immeasurably grateful for the opportunity to do this $\mathrm{PhD}$, and for the growth that I have experienced as a result of it. For this, I thank every one of you who has walked even just a part of this journey with me. To the trio of spectacular human beings who provided my supervision: Prof. Vanessa Green, Dr. Mary Jane Shuker, and Dr. Michael Johnston, I could not have wished for more engaged guidance. Your interest in this project (and encouragement to cross the finishing line) has been overwhelming. I cannot tell you how much I have come to value your friendship, wisdom, and mentoring. Thank you.

My sincere gratitude goes to the teachers and children who participated in this work. Your openness in sharing your thoughts and your willingness in allowing me into your space is something I will remember for this lifetime. It would be impossible to measure how much I have learnt from each of you. Thank you. Thank you. Thank you.

With much love, I acknowledge my family (in alphabetical order, not order of importance): Justin, Maya, Quinn, Roger, Sarah, Scott, and Sue. You never once doubted that I could do this. Mum and Dad, I especially thank you for the value that you have always placed on new learning and the importance of staring a difficult task straight in the eye. You prepared me well! And Justin, Quinn and Maya, thank you for your continual encouragement, patience, and understanding. 
I would like to thank Victoria University for two Advanced Degree Awards for writing the PhD proposal and for gathering data. Thank you also to my education sector colleagues and friends for providing opportunities to speak about my work, and for the feedback and encouragement that you have given me along the way. A special thank you goes to Sola Freeman and Tegan Lynch. Dr. Dalice Sim, thank you for checking the final iteration of the survey and for your feedback. Christine Lock, thank you so much for generously sharing your incredible knowledge. Susan Kaiser, I thank you so much for your uplifting emails in the final weeks, and for your amazing editing and referencing skills.

To my friends outside of the education sector (you know who you are), thank you for patiently listening to me talk about my studies- and mostly only my studiesfor the past few years. I know it must have seemed boring at times; however, you did a beautiful job of looking interested and making intelligent comments at just the right moments.

It is important to acknowledge the three examiners. Your comments were thought provoking, insightful, and well-received. The viva was one of the highlights of this whole PhD process for me, and I am very grateful to each of you, and the chair, for making the conclusion to this journey such an exciting and enjoyable one.

Finally, thank you to Dr. Dale Dodd, for your gentle wisdom and wry humour. I could not have done this without you. Truly. You will be (are) greatly missed. 


\section{SELECTED PAPERS AND PRESENTATIONS FROM THE THESIS}

Mortlock, A. (2013), October. Being a part or being apart: The politics of being chosen at mat time. Keynote address to the Central Kids Conference, Heritage Hotel, Rotorua.

Mortlock, A. (2013), May. Friendship and favouritism. Key note address to the Kidicorp Regional Conference, Vector Arena, Auckland.

Mortlock, A. (2014). Children's narratives at show-and-tell: What do story books tell us about 'being known', 'being better,' and 'being judged?' He Kupu, 3(5), 3845.

http://www.hekupu.ac.nz/Journal\%20files/Issue5\%20November\%202014/6\%2 0Anita\%20Mortlock.pdf

Mortlock, A. (2014), May. Friendship at mat time: The shadowy underground of children's popularity. Keynote address to the Autumn Research Seminar, Victoria University of Wellington Faculty of Education, Karori.

Mortlock, A. (2014), July. Researching your practice: Learning from an exploration into mat time. Wellington Regional Kindergarten Association Conference, Michael Fowler Centre, Wellington.

Mortlock, A., Green, V., Shuker, M.J., \& Johnston, M. (2014). Working together at mat time: Politics of participation. New Zealand Journal of Teachers' Work, 11(2), 187-200. https://teachworkojs.aut.ac.nz/autojs/index.php/nzjtw/article/view/4 


\section{TABLE OF CONTENTS}

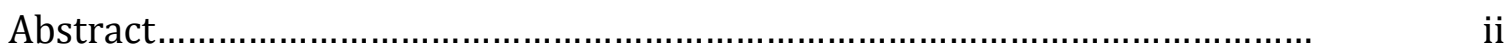

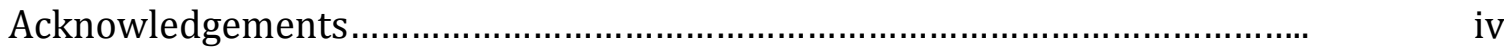

Selected Presentations and Papers from the Thesis........................................ vi

List of Tables.................................................................................................. xii

List of Figures............................................................................................

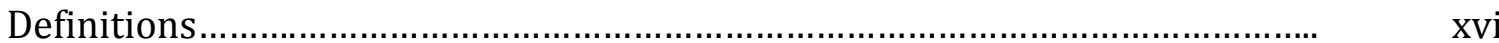

Overview of the thesis..................................................................................

$\begin{array}{lll}\text { Chapter One } & \text { Pedagogy } & 1\end{array}$

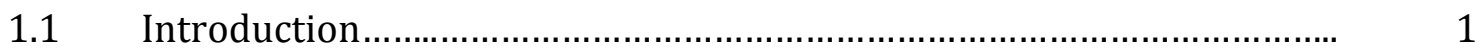

1.2 The pedagogical continuum ...........................................................

1.3 The complexities of effective pedagogy …………...............................

1.4 Teaching students as a whole group ..................................................... 10

1.5 Whole group pedagogy using the mat................................................. 12

1.6 Mat time in New Zealand........................................................... 22

$1.7 \quad$ Chapter summary and conclusions..................................................... 23

$\begin{array}{lll}\text { Chapter Two } & \text { Children's Social Worlds } & \mathbf{2 8}\end{array}$

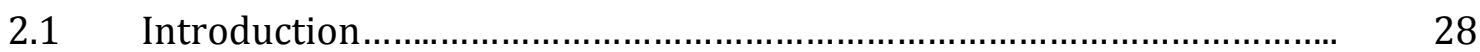

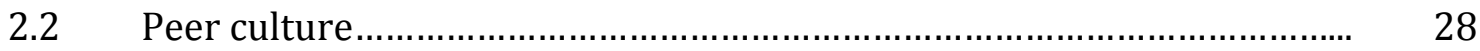

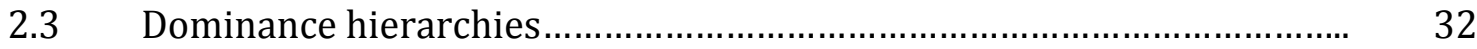

$2.4 \quad$ Friendship.............................................................................. 37

$2.5 \quad$ Children's social worlds on the mat..................................................... 40

2.6 Chapter Summary and conclusions.................................................... 42 
Chapter Three

\section{Contextualising the Research} Programme

Introduction ..........................................................................

3.2 Teachers' and children's perspectives of the mat.................................... 45

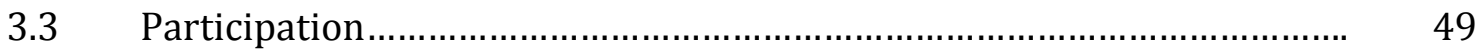

3.4 The interactional milieu of the mat....................................................... 51

3.5 New Zealand primary classrooms ..................................................... 55

3.6 Statement of the problem and purpose of the study ............................... 56

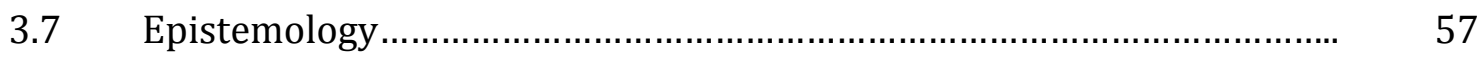

3.8 The research question and methods.................................................... 57

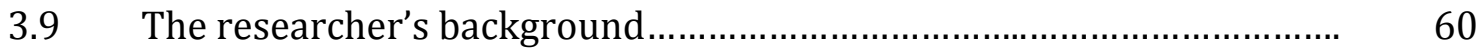

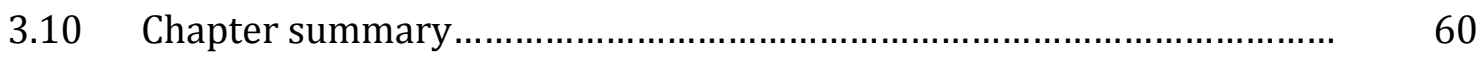

$\begin{array}{lll}\text { Chapter Four } & \text { Study One }\end{array}$

$4.1 \quad$ Introduction.............................................................................. 62

4.2 Study aims and research questions...................................................... 64

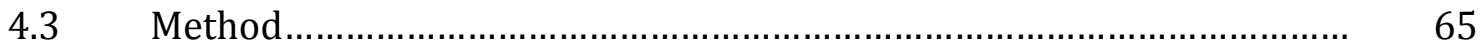

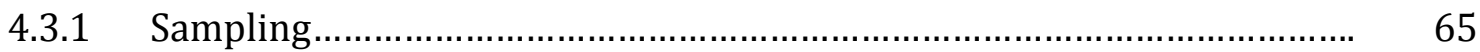

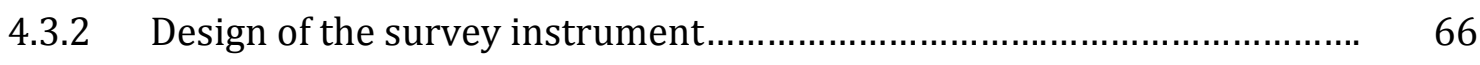

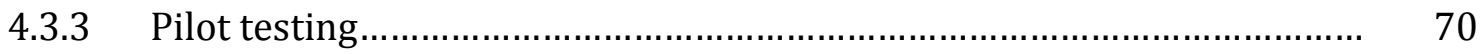

4.3.4 Administration of the survey ..............................................................

4.3.5 Data analysis strategies...............................................................

$4.4 \quad$ Results...................................................................................... 72

4.4.1 Response rate and demographic characteristics of respondents........... 72

4.4.2 Analysis of comments section ............................................................

4.4.3 Time spent on the mat...................................................................

4.4.4 Activities at mat time.......................................................................... 
4.4.5 Pedagogical goals at mat time......................................................... 80

4.4.6 Children's behaviours and feelings..................................................... 82

4.4.7 Strategies for engaging children...................................................... 84

4.4.8 Degree of challenge ...................................................................... 87

4.4.9 Pedagogical approaches at mat time................................................. 89

4.4.10 Likelihood of all children participating ................................................ 91

4.4.11 Use of children's interests and comparison of ratings of children's

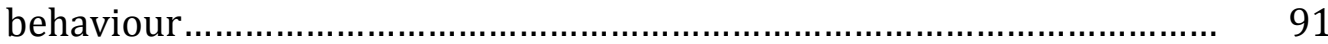

4.4.12 Use of children's interests and comparison of the degree of challenge presented by potential tensions............................................................ 94

4.4.13 Use of children's interests and comparison of teaching approach.......... 95

4.4.14 Use of interests and comparison of ratings of likelihood that all children participate

4.4.15 Comparison of teachers' ratings of the likelihood that all children participate with (1) the pedagogical approach used, and (2) children's behaviours at mat time

$4.5 \quad$ Summary of findings....................................................................... 101

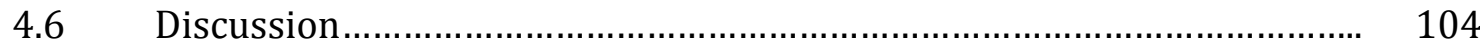

4.6.1 Amount of time spent on the mat..................................................... 104

4.6.2 Contributions of mat time to children's learning................................. 104

4.6.3 Pedagogies associated with the mat.................................................... 106

4.6.4 Teachers' perspectives of children's engagement and participation..... 107

4.6.5 The mat as an official space.......................................................... 113

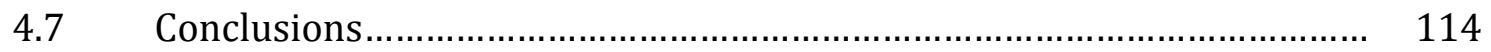

$\begin{array}{lll}\text { Chapter Five } & \text { Study Two } & 117\end{array}$

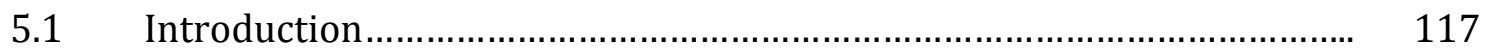

$5.2 \quad$ Study aims and research questions ..................................................... 118

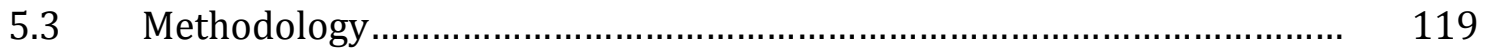

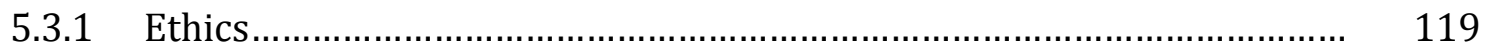

$5.3 .2 \quad$ Selection of classrooms ............................................................... 122 
5.3.3 Selection of participants............................................................. 124

5.3.4 Development of the semi-structured interview schedule and

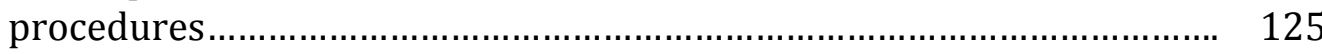

5.3.5 Piloting the interview ..................................................................... 128

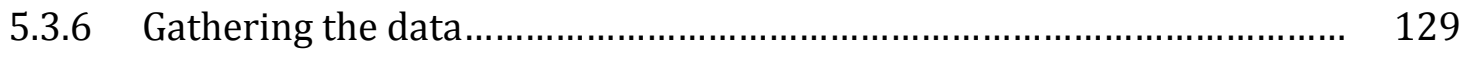

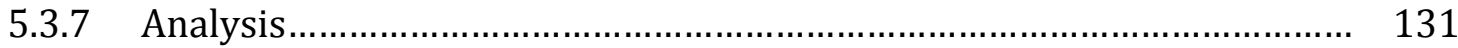

5.3.8 Trustworthiness and ecological validity .............................................. 133

$5.4 \quad$ Results................................................................................. 134

5.4.1 Seating position......................................................................... 135

5.4.2 What usually happens at mat time? ................................................. 137

5.4.3 Children's feelings about mat time..................................................... 142

5.4.4 Importance of participation ............................................................ 153

5.4.5 A closer view of children who rated their participation as unimportant 164

5.4.6 A closer view of children who referred to competition with enjoyment 166

$5.5 \quad$ Summary of findings.......................................................................... 169

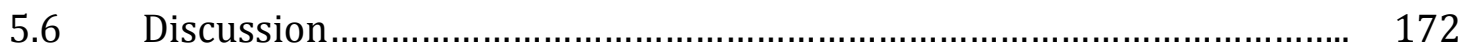

5.6.1 Children's impressions of mat time.................................................. 172

5.6.2 Children's views on participation and engagement at mat time............. 173

5.6.3 Children's social worlds at mat time.................................................. 175

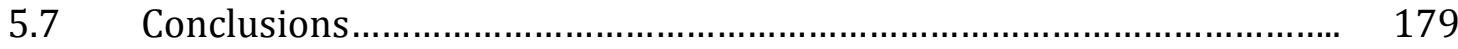

$\begin{array}{lll}\text { Chapter Six } & \text { Discussion } & 181\end{array}$

$6.1 \quad$ Introduction .......................................................................... 181

6.2 Summary of study one findings........................................................ 181

6.3 Summary of study two findings........................................................... 185

6.4 The interactional milieu at mat time................................................... 189

6.4.1 Limited resources.......................................................................... 190

6.4.2 Group cohesion and self-esteem........................................................ 196

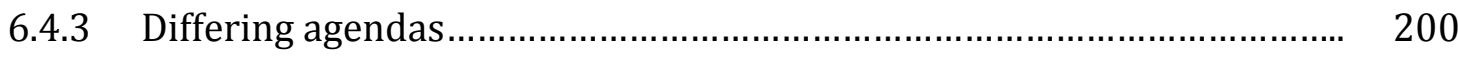


6.4.5 Why did children participate or not?........................................................... 208

6.5 A case for distributing participation.............................................. 209

6.5.1 Using children's interests to encourage participation ............................ 209

6.5.2 Distributed participation to increase engagement............................... 211

6.5.3 Distributing participation through small groups................................. 213

6.5.4 Teachers' selection of small groups................................................ 214

6.5.5 Collaborative small group interactions................................................. 216

6.5.6 Recommendations to enhance children's participation at mat time..... 217

6.6 Limitations and future directions.............................................. 219

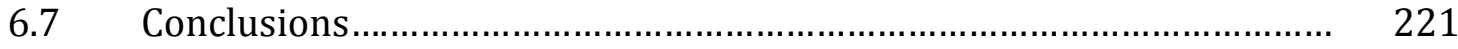

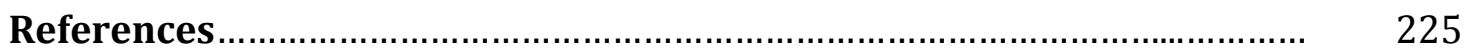

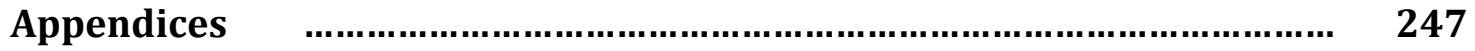

Appendix A Survey................................................................... 247

Appendix B $\quad$ Ethics approval letter..................................................... 253

Appendix C $\quad$ Information Letters.................................................... 254

Appendix D Confidentiality agreement for transcription.......................... 263

Appendix E Coding instructions to establish inter-rater reliability.......... 264 


\section{LIST OF TABLES}

Table

$1.1 \quad$ Classroom Pedagogy Represented as a Continuum.............................. 2

4.1 Distribution of the Demographic Characteristics of the Sample and the Characteristics of Respondents' Schools.

4.2 Numbers and Average Lengths of Typical Mat Times as Percentages of All Respondents

4.3 The Three Principal Components, Depicting Loadings $>.3$, for the Mat Time Activities Item Comprising of Respondents' Reports on the Frequency with which they Facilitate Social and Academic Activities at Mat Time.....

4.4 The Single Principal Component Principal, Depicting Loadings >.3, for the Pedagogical Goals Item Comprising Respondents' Agreement that Mat Time Contributes to the Achievement of Specific Pedagogical Goals

4.5 The Two Principal Components Depicting Loadings $>.3$, for the Item on Children's Behaviours and Feelings at Mat Time Comprising Teachers' Agreement to Observable Behaviours and Positive Feelings

4.6 The Two Principal Components Depicting Loadings $>.3$, For the Item on Teachers' Reports on Their Use of Behaviourist Strategies and Appealing to Children's Attentiveness in Order to Engage them at Mat Time.

4.7 The Single Principle Component, Depicting Loadings $>.3$, for the Item on Teachers' Reports of Degree of Challenged Experienced in Resolving Pedagogical Tensions at Mat Time.....

4.8 The Single Principal Component, Depicting Loadings $>.3$, for the Respondents' Reports on the Pedagogical Approaches Used at Mat Time

5.1 Coding of Children's Reasons for Seating Position Preferences.

5.2 Coding of Children's Reports about What Happens at Mat Time..........

5.3 Coding of Children's Reports on Aspects of Mat Time that are Enjoyable. 
5.4 Coding of Children's Reports on Aspects of Mat Time that were not Enjoyable

5.5 Coding of Children's Reasons for Participation.

5.6 Coding of Children's Reasons for Participation being Not Important......

5.7 Overview of Responses for children who rated their Participation as Not Important.

5.8 Overview of Responses of Children who indicated that they are Confident at Mat Time.

\section{LIST OF FIGURES}

Figure

1.1 Skinner et al.'s, (1990) Motivational model of relations among context, self, action, and outcomes.................................................

3.1 The interactional milieu as an intersection between the official space and children's social worlds.

4.1 Respondents' use of activities presented as percentages with error margins, and with activities shown in the order to which they comprise the first principle component for mat time activities: the social climate

4.2 Respondents' use of activities presented as percentages with error margins, and with activities shown in the order to which they comprise the second principle component for mat time activities: teaching the curriculum.

4.3 Respondents' use of activities presented as percentages with error margins, with activities shown in the order to which they comprise the third principal component for mat time activities: social niceties

4.4 Respondents' agreement that mat time achieves pedagogical goals, presented as percentages with error margins, with pedagogical goals shown in the order to which they comprise the single principal component. 
4.5 Respondents' agreement on children's behaviours and feelings at mat time depicted as percentages with error margins, with respondents' agreement on shown in the order to which they comprise the first principal component: observable behaviours.

4.6 Respondents' agreement on children's behaviours and feelings at mat time depicted as percentages with error margins, and with respondents' agreement on shown in the order to which they comprise the second principal component: positive feelings......

4.7 The respondents' use of strategies to engage children at mat time depicted as percentages of responses with error margins, and shown in the order to which they comprise the first principal component: behavioural reinforcement

4.8 The respondents' use of strategies to engage children at mat time depicted as percentages of responses with error margins, and shown in the order to which they comprise the second principal component: appealing to children's interests.

4.9 The respondents' ratings of the degree of challenge associated with pedagogical tensions, depicted as percentages of responses with error margins, and shown in the order to which they comprise the single principle component

4.10 Respondents' rating of their pedagogical approaches at mat time, presented as percentages with error margins, and shown in the order to which they comprise the single principal component: pedagogical approaches

4.11 Respondents ratings on the likelihood that all children participate at mat time, depicted as percentages with error margins.

4.12 Results of the paired sample $t$-tests: Comparison of teachers' reports of children's observable behaviours (observing, disrupting, dominating, putting hands up) and positive feelings (enjoyment and attentiveness), depicting the differences between those who report appealing to children's interests and those who do not.....

4.13 Results of paired sample $t$-tests: Comparison of teachers' reports on whether pedagogical tensions at mat time are challenging to resolve, depicting the differences between those who report appealing to children's interests and those who do not. 


\section{Figures continued}

4.14 Comparison of teachers' reports on their pedagogical approaches at mat time and whether they appeal to children's interests or not.......

4.15 Chi test results on teachers' ratings of the likelihood that all children participate, depicted as frequency of responses with error margins, and comparing teachers who report appealing to children's interests and those who do not....

4.16 Respondents' ratings of the likelihood of all children participating at and their agreement that children utilise differing participatory behaviours at mat time (some children consistently observing, dominating, disrupting, or putting their hand up)....

4.17 Respondents' ratings of the likelihood of all children participating at mat time and their agreement that children enjoy mat time and are attentive (children's positive feelings)

5.1 Human-like figures used as props in the interviews........................... 126

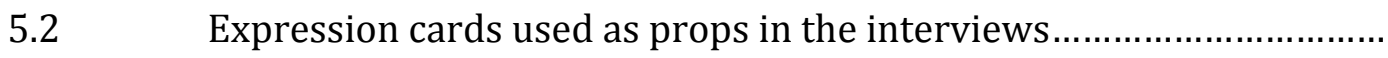

5.3 Rua's map of seating positions on the mat in relation to the likelihood of being chosen by the teacher for active participation...... 


\section{DEFINITIONS}

En bloc

En dehors

Goodness-of-fit

Group cohesion

Interactional milieu
Defined by the Collins French Dictionary as being assembled as a body, assembled together as a block (Sinclair Knight, 1995).

The literal French meaning is outside or apart (Sinclair Knight, 1995); however, in contemporary musical terms, en dehors refers to a melody that is emphasised or highlighted within a musical score (Kennedy, Kennedy, \& RutherfordJohnson, 2013). This thesis borrows from both of these meanings to describe an action that simultaneously promotes the attributes of some group members, thereby making them prominent within the group, while rendering other group members as invisible or invalid.

A term originally used by Thomas and Cress (1977) to describe the contribution that children and parents jointly make to the parent-child relationship. This thesis follows Churchill's (2003) use that widens the original definition to include the congruence between a child's social capital, attitudes, and temperament and the demands of the educational setting.

The term has been used at least since 1937, when Moreno and Jennings described group cohesion as, "the forces holding the individuals within the groupings in which they are" (p.371, cited in Mudrack, 1989, p. 41). Contemporarily, it is used to construe the safety of the emotional climate for the group members, and presence of a sense of togetherness and healthy interpersonal relational bonds (Treadwell, Reisch, Travaglini, \& Kumar, 2011).

Used in the present research to describe the interlocking space between the children's social worlds and the teachersanctioned norms, values, and practices of the classroom. The interactional milieu is influenced by: the physical context it is contained within, teachers' pedagogy, individual children's interests and agendas, and the relative power of the classroom members. 
IRF sequence

$N Z C F$

Official space

Participation

Pedagogy

Social World
A whole group teaching strategy described by Cazden (2001), whereby a teacher asks the class a question (initiates), chooses one child to respond, and then issues some kind of evaluative statement as feedback.

The New Zealand Curriculum Framework (Ministry of Education, 2007).

Described by Woodrow (2006) as the authority and control of a teacher as seen in the practices, structure and values expressed through a teacher's pedagogy.

In this context, used as an individual's ability to: actively take part, influence the group, and access a sense of belonging and support within the group (Sandberg \& Eriksson, 2011).

Act of teaching, to lead the child (Papatheodorou, 2009).

Used in the present research to describe the combined effect of children's peer culture, peer relations, friendships, and internal hierarchies. 


\section{OVERVIEW OF THE THESIS}

\section{Chapter One}

Pedagogy in junior classrooms is described. This chapter is premised on the notion that whole group teaching is extremely complex, more so than much of the literature contends. One context for whole group teaching is chosen for the focus of the thesis, which is the classroom mat. Literature about pedagogy using the mat is reviewed and it is argued that the classroom climate influences children's opportunities for learning on the mat.

\section{Chapter Two}

The second chapter acknowledges that learning in a large group is influenced by social dynamics and interactions. However, there is a growing body of research that indicates that groups of children behave in hierarchical ways. Specifically, some children will have greater status within the group than their peers. Chapter Two reviews literature that explains children's social worlds, and suggests that children's social lives might impact on specific children's participation in wholegroup activities. The chapter concludes with a brief review of some of the relational aspects of learning on the mat.

\section{Chapter Three}

The present research programme is contextualised, theoretically and in terms of the educational setting; specifically, it suggests that teachers' pedagogy and children's social worlds intersect in order to create a shared interactional milieu in which the classroom members participate. It is within this interactional space that the present thesis is conceptually situated. Finally, the present research was undertaken in New Zealand junior primary schools and this educational context is described in relation to the New Zealand Curriculum Framework (Ministry of Education, 2007). The overarching research questions are presented and explained. 


\section{Chapter Four}

The first of two studies is reported on. This study was a quantitative questionnaire that investigated teachers' perceptions of teaching the whole class on the mat. The complete study is described, including the participants and research strategies. A brief discussion of the findings is given.

\section{Chapter Five}

The second study is also reported as a discrete study and investigates the perspectives of some of the children in three different year two classrooms. The data were gathered through qualitative semi-structured interviews, and peerrelations theories were used to frame the analyses.

\section{Chapter Six}

Finally, Chapter Six summarises the two studies and discusses the findings of each in relation to the research questions. A key recommendation for mat time practices is that teachers should emphasise distributed participation in ways that protect vulnerable children, academically and socially. 


\section{CHAPTER ONE \\ Pedagogy}

\subsection{Introduction}

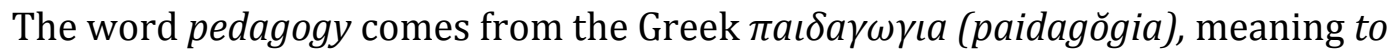
lead the child (Papatheodorou, 2009). This definition implies that teachers have a responsibility to guide a child with careful consideration and insight; consequently, there is merit to examining the nature of that guidance and how children experience it.

The present research is situated in the junior classroom context. As an entry point to this context, traditional child-centred and teacher-directed approaches to pedagogy are discussed. Whereas the first section examines pedagogy in relation to individuals, the next section examines whole-group pedagogy. Indeed, many teachers work with large groups. Unsurprisingly, several authors have raised concerns about the efficacy of whole-group teaching for all students, because any class of students comprises diverse, heterogeneous members with varying needs and understandings (Alton-Lee, 2003; Galton, Hargreaves, \& Pell, 2009; Myhill, 2002). The chapter concludes with a review of the literature about teaching the whole group of children specifically on the mat, the carpeted area of a classroom.

\subsection{The pedagogical continuum}

One commonly held view of teaching is that it is a continuum that situates teacher-directed strategies at one pole, and student-centredness at the other (see Arthur, Beecher, Death, Farmer, \& Dockett, 2014; Frieberg \& Driscoll, 1996; Rogers \& Frieberg, 1994; Stipek, 1991). At the centre, sit pedagogical strategies that incorporate aspects of teacher-directed approaches with those that are childcentred. These ideas are summarised in Table 1.1. 
Table 1.1. Classroom Pedagogy Represented as a Continuum

\begin{tabular}{|c|c|c|c|}
\hline Continuum & Teacher-directed & & Child-centred \\
\hline $\begin{array}{l}\text { Focus } \\
\text { (Fallace, 2015) }\end{array}$ & Teacher-defined objectives & & The Student \\
\hline $\begin{array}{l}\text { What } \\
\text { (Content, } \\
\text { curriculum) } \\
\text { (Killen, 2012) }\end{array}$ & $\begin{array}{l}\text { Domain knowledge } \\
\text { determined by the teacher } \\
\text { Class goals set by the } \\
\text { teacher }\end{array}$ & & $\begin{array}{r}\begin{array}{r}\text { Students determine topic/ } \\
\text { focus }\end{array} \\
\text { Students establish goals }\end{array}$ \\
\hline $\begin{array}{l}\text { How } \\
\text { (Teaching } \\
\text { strategies) } \\
\text { (Rogers \& } \\
\text { Frieberg, 1994) }\end{array}$ & $\begin{array}{l}\text { Explicit instruction } \\
\text { Demonstration } \\
\text { Closed questioning } \\
\text { Reinforcement, modelling } \\
\text { Establishing rules, use of } \\
\text { discipline }\end{array}$ & $\begin{array}{l}\text { Group discussion } \\
\begin{array}{l}\text { Cooperative group } \\
\text { learning }\end{array} \\
\text { Guided discovery }\end{array}$ & $\begin{array}{r}\text { Students explore } \\
\text { independently from teacher } \\
\text { Inquiry-based experiences } \\
\text { student-directed projects, } \\
\text { child-led learning. }\end{array}$ \\
\hline $\begin{array}{l}\text { Function } \\
\text { (Muijs \& } \\
\text { Reynolds, 2005) }\end{array}$ & $\begin{array}{l}\text { Education outcomes linked } \\
\text { to domain knowledge }\end{array}$ & & $\begin{array}{l}\text { Support of students' identity } \\
\text { Co-construction of knowledge } \\
\text { Intrinsic motivation and self- } \\
\text { efficacy }\end{array}$ \\
\hline
\end{tabular}

Teacher-directed practices accentuate teachers' decisions about what and how a student will learn. According to Garrett (2008) and Puriola (2006), traditional views of teacher-directedness place teachers as central to a lesson, often reflected by their physical position at the front of the class and the degree of control over students that is exercised. Frieberg and Driscoll (1996) define three common strategies associated with teacher-directed pedagogy: explicit instruction, demonstration, and closed questioning. Explicit instruction describes a lecture or presentation format; in other words, the teacher imparts information to the students. A demonstration is similar to explicit instruction, except that the teacher models or illustrates the application of concepts, processes, or instructions while the students observe. Finally, closed questioning elicits simple responses from 
students such as 'yes' or 'no', or the recall of information. Generally, closed questioning requires one word or short-phrase answers and appears to be commonly associated with explicit instruction. The primary purpose of closed questions is to reinforce the knowledge that the teacher wishes to emphasise (Frieberg \& Driscoll, 1996; Killen, 2012; Rogers \& Frieberg, 1994).

In contrast, Carl Rogers argued that student-centred approaches should be the core of pedagogy. He was critical of teacher-directed approaches, arguing that their tendency to promote prescribed curriculum, compulsory and uniform tasks, and use of instruction might fail to carry meaning for students and threaten their positive sense of self (1969, cited in Duchesne, McMaugh, Bochner, \& Krause, 2013). Indeed, contemporary student-centred approaches reflect Rogers' view that the individual needs to be free to explore their own interests rather than having a curriculum imposed upon them (Killen, 2012).

In further support of a student-centred view over a teacher directed-approach, Fallace (2015) asserts, "the child, rather than the teacher or textbook should be the major focus of the classroom" (p. 74). Indeed, Arthur at al. (2014) posited that a student-centred approach allows the student, rather than the teacher, to take the central role in the process of learning. This view is evident in the types of activities that are associated with student-centred strategies such as students' independent exploration, reflective inquiry, self-assessment, and self-directed projects (Frieberg \& Driscoll, 1996; Killen, 2012; Rogers \& Frieberg, 1994).

The teaching curriculum, however, does not merely comprise two poles; rather pedagogical activities fall along a continuum that blend teacher-directedness with student-centredness. When we consider how lessons are constructed, we can see that such blended activities fall in the middle, and include group discussion, cooperative group learning, and guided discovery. For example, with regard to group discussion, a teacher typically establishes a topic or question and fields 
input from the students. It is seen as an interactive forum in which students explore ideas alongside others. Students' participation is considered to include talking and listening, and both are seen to add value to learning. Furthermore, this might be one of the prevalent forms of pedagogy in classrooms (Rogers \& Frieberg, 1994).

Another pedagogical strategy that incorporates aspects of both teacher- and student-centred pedagogy is cooperative learning. Whereas group discussion typically refers to the entire class, cooperative group learning describes the practice of breaking a class up into working groups. Brown and Thomson (2000) noted that teachers should set goals for these groups and articulate expectations for students' prosocial engagement. For instance, students are typically expected to deal with any arising conflict assertively and with kindness, to respectfully consider the opinions and ideas of peers, and to act in inclusive ways.

Furthermore, Brown and Thomson cite over four hundred studies indicating that cooperative learning seeks to mitigate the competition evident in whole group discussion (such as children jousting for attention), and that cooperative group work can enhance students' academic and social skills.

The last strategy, situated in the middle of the continuum, is guided discovery, which describes an activity-based experience in which students participate in practical exploration of a topic or item that the teacher has prepared. The teacher provides provocations that guide students' inquiry toward achieving a predetermined goal. Students' engagement in the task and responsibility for following the teacher's instructions are seen as pivotal to the effectiveness of the activity (Eggen \& Kauchak, 2006).

While conceptualising teacher- and student-centred pedgogies on a continuum, rather than as a dichotomy, some scholars (for example, Stephenson, 2008) have worried that even a continuum presents a simplistic view of teaching. During a 
single lesson, teaching strategies could rapidly switch between teacherdirectedness and student-centredness multiple times. Furthermore, teaching focus can be differentiated into content and strategies, each of which can be situated simultaneously in a different place to the other on the teaching continuum. Thus, a single activity can be teacher-directed and student-centred. For example, a teacher might let students choose their own topic to research (content), which could be considered to be student-centred. However, the teacher might also issue a set of very explicit instructions about how the topic is to be researched and reported (strategy), which would be teacher-directed.

\subsection{The complexities of effective pedagogy}

Effective pedagogy should be considered in terms of how attuned it is to individual students. In other words, the effectiveness of specific strategies must be viewed in light of the contexts and situations within which they are used. As Eggen and Kauchak (2006) note, "research consistently demonstrates that there is no one best way to teach. Teachers themselves, the type of students, and the topics being taught all influence what will be most effective in producing learning" (p. 21).

Eggen and Kauchak (2006) propose that teachers must draw on several teaching models, depending on which is the best fit for a given situation. These models include the group interaction model (cooperative group work), the inductive model (guided discovery), the problem-solving model (in which students investigate and propose solutions for a given problem), and the direct-instruction model (explicit instruction). However, the efficacy of these models is dependent on a teacher's ability. Specifically, he or she must choose teaching strategies that enable a student to sufficiently master specific knowledge or skills in a given moment. Effective pedagogy, therefore, implies that a teacher could utilise any number of strategies in a given situation; however, some are more likely to be appropriate to that situation than others (McGee \& Fraser, 2012). 
Alongside these considerations is a notion, central to contemporary pedagogy, that students "shape their own minds" (Adams, 2006, p. 245). In other words, students construct knowledge as they interact with others and with contexts (Garrett, 2008). As such, learning is viewed as an active state. This is reflected in the work of Johnston, Chater, and Bell (2002), who maintain that, "the role of the teacher can be said to be part of a learning partnership... The partnership is one where all parties are actively engaged in learning and interaction" (p. 6). One aspect of this partnership involves a teacher's appreciation that students learn at different rates and in various ways according to particular life experiences, unique abilities, and psychological aspects. This includes the multifaceted and sometimes contradictory aspects of a student's prior knowledge, skills, and attitudes. It also includes the students' ability to take risks in their learning as well as their sense of self-efficacy (McGee \& Fraser, 2012). In other words, a teacher attempts to partner with a student in ways that are cognisant of that student's skills, abilities, knowledge, and ways of being.

In addition to focusing on the individual makeup of students, teachers must also lift students' competence in subjects or domains of knowledge that are deemed important by society, or by Government and its agencies. As such, students are expected to have a degree of competence in these domains in order to be contributing members of that society. Another way of putting it is that many domains of knowledge comprise concepts, procedures or epistemologies that are accepted and reproduced by a specific society. Sometimes, these knowledge and skills are difficult to learn; therefore, the attunement between the teacher and student is key (Killen, 2012). In fact, the teacher might prevent an unnecessary struggle for a student by clearly presenting core ideas. Geddis (1996) provides an apt illustration of the importance of pedagogy that is attuned to a student's understanding of domain knowledge in saying that it would be absurd to leave "children on their own to devise scientific perspectives that have taken the human 
race centuries to articulate" (p. 254). Overall, a teacher would ideally consider learning outcomes and the needs of students, rather than either one or the other.

Carr (2001) uses of the metaphor of a dance to describe those interactions between teachers and students that achieve enhanced competence. This metaphor can be related to Vygotsky's notion of scaffolding within students' zone of proximal development (1978, cited in Eggen \& Kauchak, 2006). Scaffolding is support for a student, provided by a more competent 'other', to develop greater complexity of thinking and problem solving than that student would achieve alone. As a student gains mastery, the support (or scaffold) can be gradually withdrawn. The degree of support needed for learning to occur is determined and adjusted continually during the interactions between a student and his or her teacher (White, Hayes, \& Livesey, 2013).

In order to scaffold a student effectively, a teacher must first have some understanding of a student's working theories and learning strategies. Hargreaves (2013) suggested that working theories describe how certain knowledge or understandings fit an individual student's cognitive framing, irrespective of whether he or she is factually correct or otherwise. This idea relates to Piaget's belief that even stable theories can reach disequilibrium when exposed to various provocations that challenge an individual's thinking (1985, cited in Duchesne et al., 2013). A student must, then, assimilate or accommodate new learning in order to achieve cognitive equilibrium again (White et al., 2013).

Alongside the need for teachers to be aware of a student's existing knowledge and working theories is the importance of understanding his or her strategies for achieving tasks or solving problems. This bears a direct relationship to academic performance. When a student's strategies are a poor fit for a task, teachers must assess whether he or she should guide the student toward more effective strategies. Examples can be seen in Tait-McCutcheon's (2014) research on 
mathematics pedagogy in New Zealand primary classrooms. One example describes a scenario in which the teacher asked young children to determine the number to be added to 56 so that the sum would equal 100. An inefficient strategy was counting on in ones (i.e., 57,58,59...). In contrast, the teacher was able to draw the children's attention to one student's more efficient strategy, which was to add $56+4+40=100$.

Students possess their own unique strategies, as well as goals and interests, and these are as significant to learning as a teacher's pedagogical proficiency. This is important, because a teacher's efforts to guide a student's knowledge and skills might be irrelevant if the student lacks motivation or is disengaged from the task. Duchesne et al. (2013) define motivation as "an internal process that energises, directs, and maintains behaviour over time" (p. 270). By contrast, engagement describes students' behavioural, emotional, and cognitive foci in relation to participating in specific tasks (Duchesne et al., 2013). For example, if a student is motivated by praise, he or she might not engage with the task when a there is low chance of noticeable success. In contrast, teachers might be able to ameliorate some aspects of low student motivation and engagement by designing tasks that provide optimal safety, risk, encouragement, challenge, and feedback. In addition, they can also use their understanding of a student's interests to design appealing tasks that ignite desire to participate (Duchesne et al., 2013). This is important given that motivation and interest each influence learning (Hidi \& Renninger, 2006).

A student's motivation and level of interest are closely associated with selfconcept. A child's self-concept has become an increasingly important consideration in education theory because self-concepts have shown statistical associations with participation, and engagement (Dennisen, Zarrett, \& Eccles, 2007; Marsh, Köller, Trautwein, Lüdtke, \& Baumert, 2005). These are embodied through the actions that appertain to task persistence, the degree of effort applied, 
and emotional states during learning activities. In turn, participation and action impacts on learning outcomes. Figure 1.1 illustrates Skinner, Wellborn and Connell's (1990) model of the relations between the learning environment (context), self-concept, action, and outcomes.

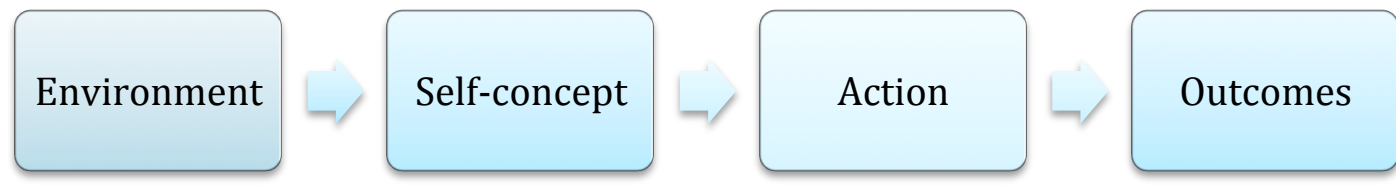

Figure 1.1: Skinner et al.'s (1990) Motivational model of relations among context, self, action, and outcomes (p. 21)

With regard to environment or context, children are cognisant of the evaluations of their academic performance made by others; they use these evaluations to construct their academic self-identities, which subsequently impact on their participation and performance in learning activities (Milman, 2009). As well as this, being able to utilise a variety of actions is core to many individuals' learning and is closely entwined with the social context of the classroom. Thus, McGee and Fraser (2012) suggest that teachers should consider ways to foster a positive social context, in which relevant key values are espoused. In other words, a teacher will talk to his or her class about social facets that optimise each student's inclusion, opportunities for participation, and abilities to take risks safely. Such a classroom context promotes students' understandings about each other and expectations about fairness. In addition, McGee and Fraser advise that a teacher provides classroom experiences that enhance students' intellectual, social, and emotional connections to each other. Overall, the social context is influenced by the behaviours of each individual that comprise the class.

Eggen and Kauchak (2006) assert that another aspect of a positive social context is the development of a "safe and orderly classroom" (p. 37). They contend that 
order is pivotal to effective teaching and learning. For instance, research suggests that teachers must establish clear rules to ensure students' on-task behaviour and other desired conduct. Furthermore, teachers, themselves, must model the desired behaviours. When students adhere to teachers' expectations, they may be reinforced, for example, by acknowledgement (see Garrett, 2008; Killen, 2012; Stephenson, 2008; Willson-Quayle, 2001). Eggen and Kauchak (2006) suggest that safe classrooms for students consist of appropriate emotional support, involvement in learning activities, opportunities to experience success, and feedback about the learning processes. Such a view implies that teachers must maintain a degree of control, whilst interacting with students in an attuned and sensitive way.

In short, effective pedagogy not only enables students to construct knowledge, skills, and understandings in relation to curriculum domains, it also protects a sense of self-competence, and appeals to motivation. However, children rarely learn in isolation from each other, and each will bring to the classroom a unique set of competencies, strategies, social roles, motivations, agendas, and selfconcepts. Therefore, the task of a teacher is to navigate the social complexity inherent when diverse individual are grouped together, and to manage the general classroom climate in ways that are transparent enough for individuals to navigate. More importantly, the context should engender positive emotional, social, and academic outcomes for each of the students.

\subsection{Teaching students as a whole group}

A case was made in the previous section that effective pedagogy involves a complex and dynamic interaction between a teacher and a student. Nonetheless, teachers have occasions in which they teach all of the children in a class as a unitary group; for example, in a class discussion or a session involving explicit instruction. Notwithstanding, pedagogical advice about whole-class situations is similar to that which is given about teaching individuals. Specifically, even though 
there might be a large number of students involved, such advice suggests that teachers should be adaptive to the students' prior knowledge, current understandings, and psychological characteristics (Freiberg \& Driscoll, 1996; Good \& Brophy, 2003; Killen, 2012; Muijs \& Reynolds, 2005). While the importance of this advice is not in dispute, concerns must be raised as to how exactly teachers are able to achieve this degree of attunement to each of their students in a whole-group scenario. This is especially so given that classes are most likely made up of students with diverse knowledge, skills, and attitudes.

Whole group teaching might have detrimental effects on specific individuals within the class. This concern is mirrored by Alton-Lee's (2003) statement that, "because teachers work with classes rather than individuals it is difficult to judge the extent to which teaching is effective for all students" (p. 8). Examples of specific children's needs going unmet are evinced in Myhill's (2002) cross-phase study of British children in junior classrooms. High-achieving children of both genders secured frequent opportunities to participate during whole-group activity. In contrast, the whole group scenario allowed far less participation for low-achieving children, and also appeared to be less effective for their learning compared with their high-achieving peers. Studies focusing on whole-group pedagogy (for example, Galton et al., 2009; Myhill, 2002) indicate that it might not be an equitable learning context.

Many of the studies about whole group pedagogy have focused on the dialogic exchanges between teachers and their students as the primary cause for concern about efficacy. Teachers often leave little opportunity for children to share their interests, opting instead for a closed-questioning style of interaction that favours academically competent students. This style of communicative exchange is often referred to as the 'IRF sequence' (Initiate, Response and Feedback). A teacher issues closed questions with an intent that a child will respond with the correct answer. The teacher then provides some kind of feedback to the child (Cazden, 
2001; Johnston, 2004; Myhill, Jones, \& Hopper, 2006; Wells, 1993). However, Raban (2001) suggests that use of the IRF sequence is not conducive to classroom discussion because it often results in any talk being mediated through the teacher's desire to arrive at a specific point. In short, whole-group activity seems to be characterised by the dominance of the teacher during instruction and discussion (see Abd-Kadir \& Hardman, 2007; Cazden, 2001; Coles, 2005; Nassaji \& Wells, 2000).

Despite suggestions of high risk of pedagogical strategies that do not meet the needs of low-achieving students and more general concerns about teaching efficacy, whole-group teaching seems commonplace. There are various theories about its prevalence, such as it being an expression of collectivity, or that teachers see it as legitimising their professional role (Rubenstein Reich, 1994).

Nonetheless, it seems possible that teaching the whole class might also be a time saving strategy for teachers. Furthermore, its use is often presented in positive ways in pedagogical texts; for example, Eggen and Kauchak (2006) indicated that well-facilitated class discussion has much value as a pedagogical strategy.

Whatever the reason, Mandel Morrow and Smith (1990) suggest that teaching the whole class will continue to be a prevailing practice. They posit that an established belief is that, "the development of the ability to learn in large groups is critical to the overall educational development of children" (p. 228). McGee and Fraser (2012) also infer that whole-group teaching is commonplace and will continue in its longevity. Notwithstanding, it was not entirely clear on what data these authors based their opinion on.

\subsection{Whole group pedagogy using the mat}

One commonly used venue for teaching the whole class together is the carpeted area of the classroom (in other words, the mat). This practice implies that there are benefits to seating the children together on the mat during whole-group activities, such as instruction or discussion, rather than tables and chairs, or in 
some other area of the classroom. From a purely practical point of view, it potentially ensures greater likelihood that children face a teacher, more so, than if they were seated around small-group tables. Another possibility is that the use of the mat is somehow thought to mitigate purported negative effects of whole group teaching. According to McGee and Fraser (2012):

Effective teachers will change classroom arrangements within lessons to suit the lesson's activities ... it is common for teachers to create a space for the whole class to sit and work together, either seated on chairs or on the floor. The latter is designated by a large carpet or rug and is usually called 'the mat'...Particularly in the early years of schooling, the mat area forms a focus for classroom activity. (pp. 131-132)

The explanation by McGee and Fraser implies that the mat enables a sense of the class working together that would not be afforded through use of other areas of the classroom. Questions abide about what is meant by 'together'. For example, the word together could imply a state of closeness that refers to either physical seating. However, McGee and Fraser seem to be also alluding to a sense of collaboration. It is not known whether there are nuances to the use of the mat that facilitate every child's participation or that instead privilege some children. In short, the extent to which literature about whole group pedagogy relates to teaching and learning on the mat is unknown.

The importance of understanding pedagogy on the mat is further emphasised when the possibility is considered that children might spend a significant proportion of their time there. One study about the design of classroom space in four junior New Zealand primary classrooms had an ancillary finding that the mat was used for between $21 \%$ and $43 \%$ of classroom time (Alsaif, 2011). 
Nonetheless, very little empirical evidence about how it is used, or what factors characterise its effective use, is available.

The literature that refers to teaching and learning on the mat describes a diverse range of pedagogical designs (Lang, 1998), which are known by several labels. One prominent approach in the United Kingdom and North America is circle time (Mosley, 1998; White, 1999), whereby children are gathered together in a circular configuration on the mat for singing and discussions. Circle time originally was designed specifically to engender a positive social climate, by focusing on children's self-esteem and sense of group cohesion. Notwithstanding, a relevant point was made by Simpson and Oh (2013), who noted that it is difficult to generalise various studies' findings on circle time because teachers often develop other foci and practices that are not related to group cohesion or self-esteem. One example they cited is doing calendar, an activity in which the date and weather are represented on a board.

Despite Simpson and Oh's (2013) assertion that circle pedagogies are varied, the majority of empirical literature about the mat has focused on the benefits of the social climate fostered specifically by circle time. For instance, Lown (2002) conducted questionnaires with 18 children in the United Kingdom, although it was unclear how old the children were. A survey was administered that required the children to respond 'yes', 'no', or 'don't know' to a range of questions about the benefits of circle time. The data suggested that circle time helped some of the children to participate more actively and to develop better knowledge of peers.

Similarly, in another study, Mary (2012) completed case studies of two French classrooms in order to investigate the role of cooperative games and circle time in fostering a positive social climate. Twelve children, aged between 7 and 12 years, took part in semi-structured individual and focus group interviews. Her findings indicated that use of cooperative games and circle time increased cooperative 
behaviours among the children. She also noted that the teachers put effort into facilitating quality communication among the children.

Both of the studies (Lown, 2002; Mary, 2012) implied that children enjoyed activity on the mat; however, despite such positive findings, another study suggested that a more cautious approach should be taken about the ascribed social benefits of circle time and children's enjoyment of it. Cefai, Ferrario, Cavioni, Carter, and Grech (2013) found that the children preferred certain aspects of circle time more so than other aspects. They enjoyed it when there was a physically active component; however, they disliked the disruptive behaviours of peers. Cefai et al.'s findings were achieved through the administration of mixedmethod questionnaires to 43 Maltese students in years three and four. The differences in reporting might be accounted for by the fact that Cefai et al. (2013) used interviews rather than questionnaire data. The interview method might allow children to add complexity to their responses by inserting caveats, for example.

A second body of studies refers to use of the mat for achieving academic outcomes, as opposed to social cohesiveness or self-esteem. One example includes the use of news (also known as show-and-tell, or sharing time). During news, children are required to share an oral monologue about an object or experience with their peers. References to this event span fifty years (see Merville, 1954) and describe a focus on the improvement of individual children's communication skills (Bohning, 1981; Merville, 1954). Other references to the use of the mat include large group time, a practice popularised by the HeadStart curricula (Hong, 1995), morning meeting (Eirich, 2006), and mat time (Klopper, 2008). In these latter practices, there is an emphasis on whole-group discussion and instruction.

With regard to the efficacy of use of the mat to children's learning, there is a dearth of studies that compare the mat with other classroom activities (for 
example, completion of worksheets). This means that few inferences can be made about the potency of teachers' use of the mat for enhancing children's learning. Just one study, Duman (2009) compared 36 children's development of academic concepts between two preschool classes in Turkey. The children were four and five years old. An experimental approach was taken whereby one class acted as a control, while in the second class, the teacher explicitly taught forty academic concepts on the mat through instruction and through props such as wall posters. Duman found that the children in the experimental class demonstrated better acquisition of the academic concepts. However, the children's parents measured the acquisition of concepts, and it was not clear what strategies were used to ensure consistency in the parents' evaluations. Furthermore, unless the two classes had the same teacher there might be a teacher-effect confound that impacted the results.

Irrespective of whether the mat is used for circle time, instruction, morning meeting, news, or mat time, it seems that the nature of pedagogy is predominantly teacher-directed. A series of small-scale studies undertaken in North American classrooms of young children aged 3 to 7 years used ethnographic approaches to explore the ways in which teachers controlled the environment on the mat (see Eirich, 2006; Ernst, 1994; Hong, 1995; Murphy, 2003). These studies used multiple methods of data collection including qualitative observations, collection of artifacts and interviews. Each found that a high degree of teacher directedness was used. This was particularly evident when teachers prioritised academic outcomes (for example, Hong, 1995). Another case was teachers' control of the children's selection of topics for discussion; meaning that teachers directed both the activity and the content (Leach \& Lewis, 2012).

Such findings about high teacher-directedness at mat time were consistent with an earlier study by Rubenstein Reich (1994) that used video-stimulated recall of three classrooms in Sweden. The intent of the study was to explore "what actually 
happens at circle time" (p. 51). Data were drawn from video observations in five preschool classrooms in different settings, and the participating children ranged in age from 1.5 years to 6.6 years. The video was shown to teachers and to an unknown number of children as part of stimulated-recall interviews. Rubenstein Reich had difficulty gaining data from the children; however, she did find from the video material that the teachers dismissed specific topics that they deemed unacceptable, even when those topics appeared to be important to the children. In this instance, teachers' pedagogical control did not appear to be well-attuned to the children's agendas.

The problematic nature of how teacher-directedness is sometimes used on the mat is evident. Teachers often appear to direct the allocation of turns for speaking (Murphy, 2003; Rubenstein Reich, 1994) and sometimes refuse to let children exercise their right to decline to speak (Leach \& Lewis, 2012). In fact, according to Danielewicz, Rogers, and Noblit (1996), children are required to "speak the language of the school modelled by the teacher, respond to the teacher's script for the event, and at times, even speak the words that the teacher insists upon" (p. 327). Such a statement underscores a belief that pedagogy that uses the mat is generally highly controlling of children.

In contrast, several studies have examined mat time when the pedagogy has been more student-centred. Specifically, these studies suggested that there could be positive benefits for children's sociolinguistic development as a result from less teacher-direction (see Danielewicz et al., 1996; Ernst, 1994; Gallas, 1992; Murphy, 2003; Poveda, 2001). Each of these studies was a small-scale ethnography undertaken in North American elementary classrooms, except the study by Poveda (2001), which researched a Spanish elementary classroom. The studies used qualitative observations as the primary strategy for gathering data. Specifically, the authors suggested that children developed better skills for speaking in groups when teachers facilitated less (Danielewicz et al., 1996; Ernst, 
1994; Murphy, 2003; Poveda, 2001). Similarly, Gallas (1992) wrote an account of teaching in her own first grade classroom. She indicated that when greater control was given to children, she reduced the corrections that she, as the teacher, made to children's speech. Not only did she believe that this enabled the dialogue to be more naturalistic, but it also resulted in children using cultural variations of speech and narration. For each of these studies, it was unclear to what extent the purported positive effects of reduced teacher control were experienced across the group of children.

The sociolinguistic studies (see Danielewicz et al., 1996; Ernst, 1994; Gallas, 1992; Murphy, 2003; Poveda, 2001) also suggested that mat time, when sensitively facilitated, might enhance the social climate in elementary classes. There was not an explicit focus on group cohesion within these studies, however. Regardless, the children in the participating studies used humour that appealed to the peer group, thus potentially enhancing a sense of camaraderie (Danielewicz et al., 1996). They also found ways to adapt their stories to what the peer group wanted (Gallas, 1992), which in turn, communicated shared interests and social norms. In addition, listeners interrupted speakers in order to make affiliative statements, for example, such as things they had in common or ways they might help each other (Danielewicz et al., 1996). They also collaborated in order to build on each other's stories (Murphy, 2003). Nevertheless, each of these studies were conducted during news or show-and-tell; the structure of which may have enabled child control more easily than, say, instruction, whereby the teacher wants to put across a specific idea. In short, while there might be social benefits to child control, the academic benefits are unknown.

Even though these qualitative studies generally posited that child-directedness at news or show-and-tell engendered positive effects on the social climate of the classroom (Danielewicz et al., 1996; Ernst, 1994; Gallas, 1992; Murphy, 2003; Poveda, 2001), other studies, of a quantitative nature, indicated that those 
behaviours associated with child-control were disruptive when the pedagogical focus used instruction or discussion. Another way of putting it is that, despite the children making interruptions and directing the dialogue during news, teachers in the qualitative sociolinguistic studies did not appear to perceive these behaviours as disruptive. In fact, those behaviours might have been viewed as essential to naturalistic patterns of group dialogue. The general assumption communicated in the findings was that the social climate was in no way negatively impacted by children's interruptions or their bids for control (Danielewicz et al., 1996; Ernst, 1994; Gallas, 1992; Murphy, 2003; Poveda, 2001). In contrast, Vargo (2008) identified children's interruptions and bids for control as being highly disruptive to the teacher. Children's disruptive behaviour on the mat was the focus of Vargo's quasi-experimental study of three to five year old children in the United States. There were 34 children in the class. Interruptions by children or their speaking out of turn made it difficult for teachers to deliver the curriculum or facilitate discussion effectively.

Several other studies, each quantitative, also conducted observations of young children's behaviour on the mat in North America and Canada, and raised concerns about specific children's disruptive actions. Hundert (2007) developed individual inclusion plans for 8 children, aged four-five years, with disabilities alongside those children's teachers. Baseline and follow up observations were taken to measure the children's on-task behaviours. Similarly, Wood, Mabry, Kretlow, Ya-Yu, and Galloway (2009) observed one class, consisting of 23 children aged five-six years, and their on-task behaviours. In addition, Ling and Barnett (2013) observed the on-task behaviours of 32 children aged three-five years and Zaghlawan and Ostrosky (2011) observed the number of challenging behaviours that an unknown number of three-five year old children made on the mat. Overall, the studies recounted disruptive behaviours such as hitting another child, calling out of turn, and leaving the mat without permission. Such behaviours distracted other children. Furthermore, some children rarely participated in the learning 
activity at hand, showing low engagement, such as looking away from the teacher or playing with equipment that was situated near the mat.

All of these studies reported high incidences of the disruptive behaviour. Vargo (2008) found that children interrupted the teacher up to six times every minute and used other disruptive behaviours at least once every minute. Similarly, Zaghlawan and Ostrosky (2011) observed children at 15-second intervals and reported that $30 \%$ of the intervals contained disruptive behaviours. Further, Ling and Barnett (2013) found that up to $94 \%$ of 15 -second intervals contained disruptive behaviours. Wood et al. (2009) observed children at five-second intervals and found that $22 \%$ of the intervals at some circle times contained children's off-task behaviour, whereas for other circle times they observed offtask behaviour in all of the intervals. Nonetheless, every study was undertaken in settings with young children aged three to five years (with the exception of Wood et al., 2009). As such, there might have been developmental issues at play that might not be apparent with older children. In short, we are not sure how older children would respond.

Other issues were evident that might have contributed to children's disruptive behaviours on the mat including teachers' responses and children's engagement. Vargo (2008) found that the teacher responded to children's interruptions, thereby positively reinforcing them. However, when she ignored children's interruptions and disruptive behaviours and responded to children with raised hands, the problem behaviours decreased. Ling and Barnett (2013) found that the use of rewards increased children's attentiveness and decreased disruptive behaviour. Their intervention consisted of a whole class approach, whereby the teacher nominated an acceptable number of distractions. If the class exhibited fewer disrupting behaviours, they were rewarded with stickers or stamps. Notwithstanding, Ling and Barnett also found that the teacher's use of positive attention increased and that she also utilised more teacher directed instruction. 
Therefore, it is unclear whether the reward or the change in teacher behaviour influenced the children's attentiveness. Finally, Zaghlawan and Ostrosky (2011) found that children's inattention and disruptive behaviour was more pronounced in certain activities compared with others; specifically children appeared more engaged during singing than in discussion. It seems, that for certain individuals at least, some activities might simply be boring.

Overall, disruptive behaviour appeared to be challenging to resolve for some teachers (Vargo, 2008); however, it was not the only challenge. Teachers experienced a range of tensions when they had multiple goals that they wanted to achieve in a single session at circle time. This is of particular importance given Rubenstein Reich's (1994) observation that coercion and discipline appear to increase with the number of goals that the teachers have to juggle. At times, goals were contradictory or incompatible; teachers had to weigh up the needs of individual children with what was best for the whole group (Rubenstein Reich, 1994). For example, Poveda (2001) described a situation in which the teacher had to maintain equilibrium between answering the students' questions about political imprisonment in Cuba while maintaining sensitivity towards two of the children with family members in prison.

Another tension arose when teachers had to balance socialisation and instruction (Gallas, 1992; Poveda, 2001). For instance, although teachers felt that sharing time or news was a social event, they appropriated children's stories to make instructional points (Poveda, 2001), or corrected children's narrative style and language (Ernst, 1994). This meant that the personable and comparatively free type of exchange usually afforded when people talk about their lives was lacking. Tensions between balancing the needs of the group with the needs of individual children were particularly evident in relation to children with special needs (Canney \& Byrne, 2006) and children with English as an additional language (Ernst, 1994; Gallas, 1992). 


\subsection{Mat time in New Zealand}

There are two studies specifically about teaching and learning on the mat in New Zealand, where the present research is situated. Both referred to it as mat time. The first study, undertaken by Gibbs and Wilks (1991), was a survey of 118 kindergarten teachers of three and four year-old children, using a range of Likert and forced-answer questions. Results were differentiated between teachers and head teachers ${ }^{1}$. Gibbs and Wilks' findings indicated a wide range of uses and aims that related to academic and social outcomes. Teachers' predominant aims were enhancing the children's sense of belonging and group cohesion, providing opportunities for group discussion, and providing opportunities for teaching.

Respondents in the Gibbs and Wilks (1991) study were asked to rank the importance of a range of pedagogical goals, including encouraging children's interests and enforcing rules and routines. Encouraging children's interests at mat time was ranked as being of high importance by $85 \%$ of head teachers and by $78 \%$ of teachers. Conversely, enforcing rules and routines was ranked as being of low importance by $98 \%$ of head teachers and by $93 \%$ of teachers (Gibbs \& Wilks, 1991). These findings suggest that the teachers used children's interests to engage them as opposed to enforcing rules that may entail strategies such as giving reprimands or rewards. Nonetheless, this study canvassed the views of teachers in kindergarten rather than in primary schools, and therefore, it is unclear whether the findings are representative of teachers' use of the mat in primary classrooms. Moreover, the study is now dated, given that there have been significant changes to the entire New Zealand education sector since 1991.

The second study was a small-scale quantitative project undertaken in a single junior primary classroom (Piters, 1995). First, the teacher rated her feelings towards individual children and then the researcher quantified and coded the mat

${ }^{1}$ Head teachers in New Zealand typically hold the leadership role in a team of kindergarten teachers. 
time interactions between the teacher and the children. Findings showed that the teacher issued more invitations for active participation to children she liked. Also, the teacher had higher expectations of this group; she felt that she would get a better quality of response from them to her questions at mat time. In other words, the teacher potentially believed that this group of children would model exemplary answers but in using them in this way, she ran the risk of positioning them as more 'clever' than their peers. As such, Piters (1995) was left with a perplexing pedagogical tension about whether all children should be given equal opportunity to actively participate at mat time irrespective of the teacher's expectations about their abilities and desire to promote particular knowledge.

Other findings indicated that the teacher issued more reprimands to children she rated as academically average. Nevertheless, Piters was unsure whether such a high level of reprimand directed at those specific children was warranted.

Because this was a small-scale project, the generalisability of its findings is in doubt. Furthermore, it is a relatively dated study given that advancements have been made to pedagogy and curriculum in primary classrooms in the last twenty years. Further discussion about contemporary New Zealand curriculum is given in Chapter Three.

\subsection{Chapter summary and conclusions}

The mat can be used for many things; however, there were three explicit uses that were prevalent in the literature. The first was for fostering group cohesion or engendering a positive social climate within the classroom. The second was for instruction and academic learning. The third use involved the sharing of narratives and discussion, and generally enhancing children's oral communication skills. In the New Zealand context, little information is available about how mat time is used, although based on the findings of Gibbs and Wilks (1991) and Piters (1995), it seems that mat time might be used predominantly for instruction and discussion. 
Overall, the research about mat time suggests that mat time is typically used for group discussion, which is typically situated in the middle of the teaching continuum, meaning that children have some agency (Rogers \& Frieberg, 1994). In contrast to the continuum, the studies in the latter sections of this present chapter suggest that discussion on the mat is actually highly teacher-directed. It was only the sociolinguistic studies that specifically explored relaxed teachercontrol in relation to news and show-and-tell (see Danielewicz et al., 1996; Ernst, 1994; Gallas, 1992; Murphy, 2003; Poveda, 2001), and that suggested that discussion could be child-directed. However, it is not known whether childcontrol could impede the delivery of information when a teachers' focus is on, say, explaining a learning activity. In the specific context of New Zealand, Piters (1995) suggested that the teacher's pedagogy was highly directed and afforded little agency to children during instruction and group discussion.

Questions abide as to whether teacher-direction is always synonymous with children's disempowerment on the mat during instruction and associated discussions. There are some pedagogical curiosities arising from conflicting findings within the overall literature. Theorists, such as Rogers (1969, cited in Duchesne et al., 2013) are critical of teacher directed, instructional activity because of its potential to be meaningless and uninteresting to students. However, researchers that report strong adult control at mat time insist that sensitively attuned control has potential to make a positive contribution to the classroom climate (Cefai et al., 2013; Lown, 2002; Mary, 2012; Moss \& Wilson, 1998; Vargo, 2008; Wood et al., 2009). Even so, other studies maintain that children should have more control at mat time in order to benefit children's language skills and the social climate (Danielewicz, et al., 1996; Ernst, 1994; Murphy, 2003; Poveda, 1991). 
The differences might lie in the type of aims that teachers wish to achieve for a specific activity; for instance if teachers wish to promote naturalistic styles of group communication, they might be more amenable to interruptions, than say, teachers who aim to teach a specific concept. Even so, it might be possible to teach specific concepts in ways that draw on child-centred strategies as well as those that are teacher-directed; for example, focusing incorporating topics or activities into the instructions that are of high interest students.

Reports of children's disinterest and disengagement at mat time are troubling. A number of international scholars were concerned about specific children's lack of engagement and on-task behaviours on the mat during instruction and discussion (see Ling \& Barnett, 2013; Rubenstein Reich, 1994; Vargo, 2008; Wood et al., 2009; Zaghlawan \& Ostrosky, 2011). However, the two New Zealand studies reported differences with regard to children's behaviour. Gibbs and Wilks (1991) found that teachers placed children's compliance to rules as a low priority, and issued few reprimands or rewards. In contrast, Piters (1995) reported that the teacher issued frequent reprimands to particular children, indicating that these children's behaviour was perceived as being disruptive or inattentive. The differences might be partly accounted for by the fact that in Gibbs and Wilks' study, teachers self-reported and the positive aspects of their practices might therefore have been augmented.

The reasons for particular children's lack of engagement and on-task behaviour is important because attentiveness has been associated with quality of learning (Eggen \& Kauchak, 2006; Hidi \& Renninger, 2006). Some studies have attributed distracted or disruptive behaviour to individual children's disabilities (see Hundert, 2007; Raver et al., 2013), and Zaghlawan and Ostrosky (2011) suggest that some activities are boring to children; however, very little information is available about strategies to enlist children's attentiveness. On balance, however, 
it is not known to what extent teachers used punishment and reward systems instead of sensitive and playful strategies to motivate children.

Another element that is not sufficiently addressed by the literature is the teachers' expectations and issues apparent for children's participation; for example, Leach and Lewis (2012) argued that teachers' practice of enforcing each child's turn to speak was in breach of the rights of children who did not want to participate. However, other studies did not indicate whether all children were expected to take part, or what kinds of participation teachers and children desired. Children's interactions with each other on the mat were comparatively unexamined compared with teacher-student interactions. More over, it was unclear the extent to which equitable access to active involvement was an issue. Teaching the whole class on the mat appears to be pedagogically complex, especially when it comes to teachers' ability to balance multiple pedagogical goals and learning needs. The general concerns raised about unequal opportunities for children's participation in any whole group activity (see Abd-Kadir \& Hardman, 2007; Cazden, 2001; Coles, 2005; Nassaji \& Wells, 2000) appear to be just as relevant for activity on the mat (Ernst, 1994; Piters, 1995; Rubenstein Reich, 1994).

Given that contemporary conceptualisations of effective education are akin to partnerships between teachers and children (Johnston et al., 2002), concerns about teachers' ability to attune to each individual child's skills, understandings, motivations, and self-concept when on the mat, are justified. If Alsaif's (2011) finding that four classrooms spent $21 \%$ to $43 \%$ of their day on the mat is generalisable to other classrooms, this means that a significant proportion of time is spent on activities whereby teachers might not be able to respond sensitively to all children's learning and emotional needs. As such, the mat, as a key arena within classroom life, is worthy of further investigation. 
In summary, several studies have suggested that teaching on the mat presents pedagogical tensions for teachers, such as balancing predetermined learning outcomes with students' interests or balancing the needs of the individual with those of the group (see Canney \& Byrne, 2006; Collins, 2013; Danielewicz et al., 1996; Gallas, 1992; Leach \& Lewis, 2012; Mary, 2012; Rubenstein Reich, 1994). The question arises as to why teachers persist with teaching on the mat, given its apparently challenging nature. Moreover, it is not known whether enabling all children's participation is challenging to teachers, or whether children find it difficult to secure participatory roles, or even whether they have a desire to actively participate. Finally, little is known about the enablers and barriers to children's participation on the mat. To date, little focus has been placed on students' peer relations or interactions with peers as a mechanism for enabling or disenabling participation on the mat. In the next chapter some of the theoretical literature and research about children's social worlds and its applicability to participation on the mat will be reviewed. 


\section{CHAPTER TWO Children's Social Worlds}

\subsection{Introduction}

In the previous chapter, education was examined in terms of teacher-child dynamics; however, many psychologists and sociologists have argued that children's ongoing interactions with each other have a significant influence on their learning, socialisation, and development (Bukowski, Buhrmester, \& Underwood, 2011). In this chapter social dynamics are discussed in the context of peer relations between students. Specifically, peers influence and actuate one another's opportunities for participation, and arguably, learning. In other words, peer interactions form a significant component, not only of children's social lives with each other, but their academic lives also (Hartup, 1996; Howes, 1983; Kindermann, 2007).

Children construct a group culture that is different to that of adults. Furthermore, peer interactions can be intensely political, as evinced by children's affiliating and competitive behaviours. Such behaviours apply to children's play cultures but are also evident within the classroom (El-Ghoroury, 2002; White, Jones, \& Sherman, 1998). In addition, friendship is an important component of children's peer culture and social status within the peer group (Corsaro, 2003). Overall, it seems likely that children's social worlds have some influence on their interactions at mat time.

\subsection{Peer culture}

Children have peer cultures that differ to those of adults, and children appropriate aspects of adult culture for their own ends. Children's peer cultures most likely have understandings, rituals, and values that are unique to them as a collective (Corsaro, 1985). This view has pertinence when we consider that children might perceive the routines and rituals associated with the mat in different ways to adults. 
Arguably, the most prevalent scholar of children's peer cultures is Corsaro (1985, 1988, 2003, 2012) whose work has been undertaken in Italy, Norway, and the United States. His ethnographic research has centred on children in early childhood settings while they are engaged in free play. By observing children in this way, Corsaro (1985) has identified that children actively construct their own peer culture, which differs to that of adults, while still remaining connected to the adult world. He suggests that a preschool offers a milieu, specific to its unique context, consisting of the physical environment and the relationships that occur within it. The interplay between the physical environment and the interpersonal environment forms a specific ecology wherein children construct shared activities, common interests, and modes of participation. Such commonalities are core to children's development of their own communities. Over time, practices within a community are adapted, negotiated, reproduced, challenged, and protected. Those that remain stable become central to the peer culture of that community (Corsaro, 1988; Corsaro \& Rizzo, 1988). Indeed, social events, social routines, and communication are pivotal to producing and maintaining children's peer cultures. Corsaro (2012) later went on to define peer culture as, "a stable set of routines, artifacts, values, and concerns that children engage in with their playmates" (p. 488).

Corsaro $(1985,2012)$ observed that children protect the boundaries of their relationships with preferred playmates within the culture of a wider peer group. Strategies used to do this include affiliating, controlling, and blocking behaviours. These are often expressed in the free play of young children. For example, Corsaro (2012) observed an episode of socio-dramatic play in which a child, Denny, took on the role of a kitten. In the game, he had two human parents: a child called Bill, and another named Rita. The 'parents' told the kitten to go to the 'backyard', but he was reluctant. The two 'parents' commanded and shouted at him, one resorting to physically pushing him. Eventually, he conceded, and moved to the 'backyard'. Rita and Bill in this case employed strategies aimed at controlling both the nature 
and direction of the game; the strategies were also an expression of status of the play role. In this event, Denny's playmates blocked his intention and his chance at equal status through their more dominant play characters, (i.e., parents). In other events, even the entry of other children into the play might be blocked so that the integrity of the players' dramatic episode is protected (Corsaro, 2003).

It is important to note that Corsaro (1985) observed that blocking techniques did not consistently occur towards any specific child. In other words, there did not appear to be rejected children within the groups that he studied. This is unusual, given that Corsaro's research is typically conducted with children in the early years, and that peer-rejection is known to begin when children are three-four years old (see Bukowski, Buhrmester, \& Underwood, 2011).

At the same time that children create their social systems, they encounter boundaries established by adults. According to Corsaro (1988), children desire control over their own lives. They might frequently find the rules imposed by adults to be arbitrary and unfair. Therefore, they gather and use information from their specific ecologies. In the process, they construct understandings about adult knowledge and skills (Corsaro, 2012; Corsaro \& Rizzo, 1988). Subsequently, they work together to challenge, subvert, or appropriate adult authority. Examples seen by Corsaro $(1995,1998)$ include children developing complex strategies to avoid assisting when asked to help tidy, or smuggling forbidden toys into their preschool.

Similarly, Milman (2009) found that some children use subversive behaviour when teachers require attentiveness. The findings arose from Milman's observational research on the ways in which 60 children and their teachers constructed notions of attentiveness in Californian first grade classrooms. The children constructed an appearance of paying attention to the teacher rather than actually being attentive (arguably a common classroom requirement). At times, this behaviour was an act of resistance for certain children, in that they were not 
really paying attention at all. Milman's interpretation was that the children had claimed their own power and control through their shared use of subterfuge.

Whereas Milman's (2009) research suggested that the children's ruse had a bonding effect, another study found that children also might use the teacher's rules and official scripts as a mechanism for furthering their own personal gain. Jordan, Cowan, and Roberts (1995) conducted qualitative observations in two kindergartens and two preschools and found that some children had a keen awareness of rules, and the association between those rules and personal agency. They stated that:

Knowledge of the rules, particularly the adult imposed rules controlling behaviour in the classroom, can be made a means for achieving power in this sense. Invocation of these rules, we discovered, is used as a discursive weapon to carry out the child's personal agendas, to control the behaviour of other children, and to prevent their own behaviour from being controlled. (p. 340)

Historically, peer culture studies see children as forming a collective identity separate from that of adults, and make comparisons of agency based on their generational age (see Olk, 2011). However, Galbraith's (2011) findings show that even within a children's collective, there is an internal vertical structuring based on status as a preferred peer. She extended Corsaro's theory of children's blocking strategies, drawing on social psychology. Based on her observations of kindergarten children at free play, Galbraith suggested that blocking strategies might have created several sub-groups, each with its own peer culture. Each subgroup developed its own rules about inclusion and affiliation that differed from those of other sub-groups. For example, one particular sub-group controlled inclusion by requiring that its members wore a cape. Owing to the scarce number of capes available, complex social rules developed to (1) enhance preferred peers' chances of possessing a cape, and (2) minimise the chances of other peers whom 
the sub-group preferred less. In this way, the capes became a "marker for affiliation" (Galbraith, 2011, p. 42). Subsequently, the sub-group rejected those children without capes.

Much of the work on peer culture has focused on early childhood settings in the context of free play. There is little research about peer culture in the classrooms of older children, especially relating to structured activity for which adults might issue a high number of rules and expectations. A second point of consideration is that peer culture studies have predominantly relied on adults' ethnographic observation and detailed recording of children's discourse. The richness of these data is not in dispute; however, there could be benefits in asking children directly about their understandings of the classroom scripts and peer culture. Such an approach would most likely necessitate trusting relationships between childparticipants and the researcher, as well as an understanding of the educational context.

\subsection{Dominance hierarchies}

Galbraith's (2011) blending of social psychology and peer culture research has merit, in that it has shown that different sub-groups of children might have qualitatively different experiences of inclusion and participation in the wider peer group. In fact, studies from various disciplines, amassed over several decades, indicate that children's peer groups consist of internal hierarchies in which individual children are afforded varying status (see Charlesworth \& La Freniere, 1983; Hawley, 1999). Many of these studies have roots in Human Sociobiology.

Historically, studies of Human Sociobiology posited that hierarchical behaviour had a genetic component and was linked to competition for dominance (Strayer \& Strayer, 1976). Lopreato (2006) noted that it was originally founded on the principles of evolutionary psychology and Darwinian theory, and suggested that certain individuals in any population are genetically better adapted for competition. In other words, it was thought that dominance was achieved by 
genetic determinism. This view suggests that once certain individuals have entered into multiple competitive situations with particular others, patterns of dominance emerge and are maintained. Indeed, Strayer and Strayer's research ranked children by dominance based on matrices of 'winners' and 'losers' in social transactions. The matrices recorded the stability of specific children's dominance by their propensity for winning. In other words, such children were effective competitors.

In addition Edelman and Omark (1973) reported that children within a group are likely to be able to identify which children are the most dominant. For instance, in their study of 450 North American children in Nursery through to Grade Three, they found that first grade children experienced little difficulty in identifying physically dominant peers when asked to name who were the 'toughest' in the class. Furthermore, Green and Rechis (2006) suggest that children who can compete effectively may become dominant or socially central to the group and take on higher status to peers in the classroom.

Dominance hierarchies may occur as children compete to acquire and maintain use of specific resources that are in short supply, and are desirable to the group. Charlesworth and La Freniere (1983) stated that the term 'resources' denotes any material object; however, it also includes goals and abstract rewards. The resources often contribute to the child's development and overall welfare. As such, authority or status, attention, and care, are considered contestable resources. Hawley (1999) asserted that human beings might compete for essential and trivial resources (for example, use of a particular coloured pencil). She suggested that an immediate association with wellbeing might not be evident when an individual is able to secure a trivial resource; however, when that trivial resource is highly desirable to a wider group, then the individual who can successfully obtain it has proven his or her dominance. 
Whereas the historical perspective of dominance was based on genetic determinism, contemporary scholars of children's hierarchies now assert that dominance is also associated with the social context. In fact, DeRosier, Cillessen, Coie, and Dodge (1994) refer to "social-psychological dimensions of the group context or atmosphere" (p. 1075). Indeed, Pellegrini et al. (2007) conducted observations of the dominance behaviour of 65 preschool children in North America. One of the common dominating strategies was the use of aggression. Their findings suggested that there were decreases in the aggression associated with resource acquisition and dominance across the year in the classrooms that had clearly espoused social rules. This means that clarity of social norms impacted the social context and therefore the hierarchical or competitive behaviours occurring within it. They stated, "A combination of group cohesion and teacher socialisation may explain the decrement of aggression across the school year" (p. 60).

Even though the social context is now thought to play a more integral role in dominance than genetics, there is still agreement that competition for resources is a fundamental component of hierarchical or competitive behaviour (see Benenson \& Heath, 2006; Hartup, Laursen, Stewart, \& Eastenson, 1988; Roy \& Benenson, 2002). What is more, Fein (2012) suggested that hierarchies are formed through the careful observation and testing of which group members possess greater strength or competitive ability in a range of domains. The domains of focus are determined by what has value to the group. In other words, it is not enough to be strong or able in a given area; the other group members must respect and recognise those attributes of an individual in order for him or her to become dominant. Moreover, the role of competition was evident in Pellegrini et al.'s (2007) findings that, "children were systematic in choosing the targets of their aggression and this choice was probably based on their belief that they could defeat their targets in competition" (p. 61). 
Some of the research about hierarchy and context has viewed teacher approval as a desired resource by the children's peer group. Teacher approval has been correlated to highly contextually subjective student behaviours such as demonstrations of 'working hard' and compliance (Mercer \& DeRosier, 2010; Wentzel \& Asher, 1995). Nonetheless, competition for teacher approval has been shown to have significant implications for children's hierarchies. For example, ElGhoroury (2002) found that negative teacher feedback in first and second grade was implicated in individual children's poor reputations and low status with peers. This study asked 183 first and second grade children in North America to rate their perception of a target actor. Similarly, White et al., (1998) asked 112 North American first and second grade children to view a video depicting several child actors and rate them on likeability. The findings indicated that negative teacher feedback caused the actors to be rated as less likeable by the participants, and being rated as more likely to engage in inappropriate behaviour. The opposite was evident when the teacher issued praise to the actors.

White and Kistner's (1992) study of 149 children in kindergarten, first, and second grades in North America also found that positive feedback by a teacher was associated with higher peer preference and better reputation within the peer group. Similar findings were reported in a second study of 490 students and 26 teachers, in kindergartens and the early grades of elementary school, in North America by Mikami, Swaim-Griggs, Reuland, and Gregory (2012). When teachers preferred academically talented children over their peers in kindergarten, first, and second grade, stronger negative effects were seen in non-preferred children's externalising behaviours (such as aggression, off-task behaviour, and disrupting peers). Accordingly, there appeared to be a link between the teachers favouring of academically talented students and a lack of social preference among the peer group for those students who did not display the desired traits (Mikami et al., 2012). These findings matter when we consider that when the class is seated on the mat, any feedback to individual children is of a relatively public nature. 
Therefore, children might appropriate the teacher's feedback to inform the status of particular peers within the peer group.

It would be erroneous to assume that all children are interested in competing for a given resource, or that they each hold similar goals in social transactions.

Renshaw and Asher (1983) opined that children differ in their goals. Furthermore, goals might change according to the situation (Ojanen, Aunola, \& Salmivalli, 2007). Overall, goals can be separated into two types. The first type comprises goals that are directed toward gaining agency or power. The second group includes goals that aim to create and reinforce affiliation and intimacy (Buhrmester, 1996). Another set of typical goals was suggested by Dijkstra, Kretschmer, Lindenberg, and Veenstra (2015); they refer to hedonic goals, which aim to achieve immediate gratification, instrumental goals, which aim to improve the child's situation, and normative goals, whereby the child aims to do something because the child thinks he or she is expected to. In short, children can be motivated by a wide range of goals when engaged in any kind of social interaction.

Each of these papers cited research with children aged 9 or older (i.e. Buhrmester, 1996; Dijkstra et al., 1996; Ojanen, et al., 2007; Renshaw and Asher, 1983). Nonetheless, the findings might be generalisable to younger children, given that Kavanaugh and McCall (1983) observed that children as young as two years might forego hedonic or instrumental motivations by sharing a toy with a peer. This would be considered an action based on either affiliative or possibly normative motivations. However, their research indicated that a playmate might not reciprocate, showing that in that moment, the playmate's actions were motivated by goals other than affiliation or adherence to norms. It is clear that each child had brought differing goals and motivations to their shared interaction. The data were gathered by observing sixteen pairs of unacquainted children at dyadic play in the United States. 
Notwithstanding the findings of Kavanaugh and McCall, further research into children's social goals is required in the later stages of early childhood, and classroom situations. Another topic warranting further investigation is young children's behaviours in classroom situations that are of a socially competitive nature. Interaction on the mat might provide a classroom forum in which children potentially compete for a range of resources such as a teacher's attention, or peer affiliation. Furthermore, children might construct understandings of their comparative dominance or social status based on competitive performances, such as giving an insightful response to a teacher's question and receiving that teacher's praise.

\subsection{Friendship}

Friendships have been implicated as playing a role in children's hierarchies and competition for resources. For instance, according to Wilson's (1975) sociobiological view, humans are self-serving and more likely to help kin than non-relatives. More recent interpretations indicate that, in situations such as classrooms in which kin are not present, children are likely to favour friends over acquaintances (see Hartup et al., 1988). Wentzel and Caldwell (1997) defined friendship as "mutually determined dyadic relationships" (p. 1198). Other explanations go further and refer to principles of cooperation and reciprocity; for example, Hartup (1996) states that friendships are "dyadic, mutually rewarding bonds between children. They are defined by reciprocity: peer bonds can be called friendships when both children gain benefits" (p. 379).

Children can also belong to friendship groups, which are a bonded series of dyadic relationships across the members of the sub-group (Wentzel \& Caldwell, 1997). Hartup (2000) and Lopreato (2006) argued that such friendship groups form when people are cognisant of specific commonalities with others in a wider group, which can be used to establish a sense of 'we-ness'. These might include shared experiences or interests, but may also include other characteristics such as ethnicity or gender. Creating the impression of commonality makes sense when 
groups that cooperate effectively (by utilising the strengths of each of its members) may be more successful in competitive situations than an individual acting alone. Charlesworth and La Freniere (1983) placed North American children in a competitive situation to view a movie. The study consisted of two groups of children aged between 4.4 and 5.5 years; the first group comprised of 18 children and the second group of 40 . At any given time, only one child could view the movie. One group of boys, who were friends in a single clique, was able to cooperate with each other in order to take over the viewing lens.

Friendships differ and not all friendships are constructive; the identity of friends and the quality of friendship matter a great deal to a child's development. Overall, friendship studies frequently measure the degree of constructiveness through five factors: (1) low conflict, (2) closeness, (3) companionship, (4) helping behaviours, and (5) security (Bukowski, Hoza, \& Boivin, 1994; Cillessen, Jiang, West, \& Laszkowski, 2005; McDonald, Wang, Menzer, Rubin, \& Booth-LaForce, 2011). Such studies suggest that low quality friendships are unlikely to have high degrees of any of the five factors. Non-constructive friendships, characterised by high-levels of coercion and conflict, have been associated with troublesome and disruptive behaviours in class (Hartup, 1996). There may be an imbalance of power whereby less dominant children feel that they must follow the whims of their more popular friend. Findings from Adler and Adler's (1995) longitudinal ethnography of North American elementary school-aged children reported instances where children unwillingly ridiculed peers at their popular friend's behest. Participation in this type of relational aggression often occurred in order to avoid becoming the focus of the popular friend's ridicule (Adler \& Adler, 1995).

Several studies indicate that low quality friendships or friendlessness may impact a child's development. For instance, Hartup, Laursen, Stewart, and Eastenson (1988) observed 53 children aged from 3.4 to 5.4 years. Friends managed conflict in gentler ways than non-friends, meaning that children could explore prosocial strategies for resolving conflict without driving the situation to a win-lose 
scenario (as was the case for non-friends). Moreover, Hartup (1996) and Howes (1983) also noted that friends provide each other with unique opportunities to practise cognitive and social skills that differ to the opportunities provided by non-friends. Indeed, they are more likely to engage in complex play (Hartup, 1996; Howes, 1983).

In contrast, friendlessness can result in lost opportunity to practise the very skills that could help children secure and maintain friendships. Thus friendless children find themselves in a bind (Howes, 1983). Moreover, Howes (1990) suggested that children interpret the actions of peers vis à vis their status and reputation. In other words, when peers disliked a child, that child had a corresponding negative reputation in the peer group; for example as 'naughty' or 'mean'. The data were gathered on 45 children who were observed from kindergarten to third grade in the North America. Assessments of popularity and friendship were made. The implications of the findings are that children might perceive a low status or a friendless peer's specific actions in a negative light based on his or her reputation. A second study by Ladd and Coleman (1997) used interviews with 102 kindergarten children with a mean age of 5.8 years. The results indicated that friendship correlated with school attitudes, in that children who disliked school were more likely to report poor peer support or social isolation. Similar findings about the correlations between low social support with children's adjustment and engagement in school were corroborated by other studies such as Buhs, Ladd, and Herald (2006) and Ladd (1990).

Much of this research on friendship has often been undertaken in laboratory or play situations. Further research into the role of children's friendships in participation in classroom activity is warranted. Given that friends might help each other's performances (Wilson, 1975; Lopreato, 2006), it follows that they might also do this during tasks within the classroom. This is of particular relevance, given that specific children most likely possess better competitive strategies in securing a participatory role or achieving their own agenda (Putallaz 
\& Sheppard, 1992; Green \& Rechis, 2006). For example, they may be more successful in forwarding their own ideas and being listened to by the group. Therefore, having the support of a friend might enhance the success of less strategic children (Bukowski et al., 2011).

\subsection{Children's social worlds on the mat}

The above sections described research undertaken in a variety of settings; however, none referred to the social dynamics of children's interactions on the mat. Few studies have connected children's social worlds to the mat. Two ethnographic studies undertaken in North American kindergartens found that particular children demonstrated a strong attachment to sitting next to friends (Kantor, Elgas, \& Fernie, 1989; Meyer et al., 1994). Two other North American ethnographic studies (Danielewicz et al., 1996; Murphy, 2003) observed young children during show-and-tell. The teacher's intention was that the children were enabled to lead the event with little adult guidance. The benefits were that relaxed teacher control enabled the peer group to introduce a higher level of peer humour and peer-relevant stories that seemed to cement a sense of togetherness among the class.

Similarly, an earlier study conducted by Moss and Wilson (1998) focused the role of cooperative activities on the mat in enhancing the social climate of a classroom. They undertook sociometric testing of 25 children in year six in a single United Kingdom classroom, to determine individual children's popularity and status. Cooperative activities were implemented that focused on relationships, and follow-up sociometric testing was administered. Opportunities for children to share their interests with each other were important for inclusion. When children could learn more about each other, they were more likely to develop amenable feelings about working with children who they ordinarily would not choose to work with. Another study, undertaken by Eirich (2006) found that a democratic orientation to morning meeting (calling for children's input and using voting) enabled children to share their interests with each other, influence the 
curriculum, and develop peer culture. Her data were gathered through her ethnographic study of 23 North American children aged six to eight years. As such, each of these studies indicated that mat time might engender children's positive interactions with each other.

Nonetheless, the shared space of children's peer culture may not always yield positive outcomes. For example, in the study by Danielewicz et al. (1996) specific children used one situation in which a teacher had relinquished control to the class to exercise power over specific peers. Other studies about interactions on the mat have noted that there is frequently a clash of values and intentions between those of the teacher and those of the children's peer group. For instance, during a game of 'musical chairs' in a Danish preschool, the children became increasingly competitive (Svinth, 2013). The teacher said, "it's just a game, it doesn't matter who wins!" (p. 1250). In other words, while the teacher's priority was on 'fun', many of the children prioritised winning. As such, several children utilised coercive or physical strategies to gain a competitive advantage, and others became distressed when they 'lost'. Similarly, Eirich (2006) observed children competing to outdo each other academically at morning meetings, despite the importance that she, as the teacher, placed on collaboration.

Other forms of dissonance between a teacher's intentions and those of specific children have been found. Cefai, Ferrario, Cavioni, Carter, and Grech (2014) administered questionnaires to children in Malta and found that teachers placed a strong onus on children sharing personal issues at circle time with an intention to enhance group cohesion. However, Cefai et al. (2014) also noted that some children would use the information shared for spreading rumours or to ridicule certain peers. A further clash in values and intentions was made evident in Wallat and Green's (1979) sociolinguistic study in one North American kindergarten. Transcripts of talk on the mat showed that some children had become adept at interrupting the teacher and taking a disproportionate number of turns to speak. Nonetheless, they did so in prosocial, non-disruptive ways such as making eye- 
contact with the teacher and sharing an appealing on-task statement. Such children had identified a 'loophole' in the explicit rule about not interrupting speakers. Their sophisticated awareness of how to appropriate and exploit the explicit rules meant that their participation was greater than that of their peers.

Leach and Lewis' (2012) findings suggested that aspects of the circle time had a divisive effect on the peer group. They conducted a focus group interview with eight children aged 10-11 years that asked them to identify the types of things they did in circle time, and what they liked and disliked about it. Specifically, Leach and Lewis found that particular children used it as a forum for reporting disputes and informing on specific peers

Each of the above studies was undertaken on the mat during circle time or showand-tell. Given that circle time and show-and-tell frequently have specific pedagogical foci, it is not known how relevant these findings are to more formal mat learning events such as instruction or group discussion about learning. Also, the research has predominantly been undertaken in early childhood settings; only four studies included school-aged children as participants (Cefai et al., 2014; Danielewicz et al., 1996; Eirich, 2006; Leach \& Lewis, 2012). Therefore, the relevance to older children has not been fully established. Finally, no studies have been conducted in New Zealand, which have examined the relationships between children's social worlds and mat time.

\subsection{Chapter summary and conclusions}

Much of the research on peer culture refers to the vertical structuring of relationships between adults and children, because of the adults' comparatively greater power and competence (Bukowski et al., 2011). The literature here, however, suggests that even within the structure of children's peer groups, there are considerable disparities in status. Indeed, Galbraith's (2011) suggestion that the peer group cannot be treated as a homogenous culture can be confirmed; the peer group consists of varied internal power dynamics. 
While much of the social psychology literature discusses social aggression and dominance, teachers might not see children behaving in generally competitive ways towards each other on the mat compared to free play. Nonetheless, an intriguing possibility is presented; this body of work suggests that children's participation on the mat might be influenced by their respective status within the hierarchy. Moreover, considering Fein's (2012) assertion that children observe the demonstrations of ability of others, and compare these against their own performance, status differences might be further reinforced. A further effect may include some children feeling reticent about participating if they perceive their performance to be substandard compared with their peers. This might cause them to avoid active participation.

Some studies suggest that social support is a key factor in successful participation. For instance, Danielewicz et al. (1996) noticed that some children looked bored and disinterested when a peer with low social support spoke; therefore, their contributions were often shortened. According to the literature on hierarchy, it is possible that the children who have social support are those children who exhibit high ability in traits that are valued by the peer group and teacher. For example, academic performance might be highly connected to social support. Other children with low social support might not have their ideas abetted by peers on the mat (Jenkins \& Kilpatrick Demaray, 2012). That friends will assist and support each other more so than non-friends is a basic premise of sociobiology.

The peer-relations literature suggests that disparate power relations exist within peer groups, whereas the peer-culture literature generally tends to look at children's power as a group in relation to that of adults. Peer-culture studies make an important contribution to the theoretical underpinning of the present research. They suggest that children and teachers will interpret structures and practices differently. There may even be fundamental clashes in values (Corsaro, 1985, 2012; Svinth, 2013). For example, Jordan et al. (1995) cited examples of children 
using a teacher's feedback as a tool for judging peers, and Svinth (2013) observed children adapting a teacher's 'fun' activity into an intensely competitive episode. For the teachers, these were certainly unintended consequences. Furthermore, teachers and children might value different aspects of learning on the mat. For instance, they might have different views about the importance of participation. Accordingly, the combined studies from the various research fields that have been included in this review indicate that it is very important to understand how children and teachers view praxis using the mat. Such a view must take into account children's peer relations. 


\section{CHAPTER THREE \\ Contextualising the Research Programme}

\subsection{Introduction}

The present research set out to investigate the perspectives of teachers and children appertaining to what happens on the mat. Whole-group teaching literature has focused on how it promulgates academic inequities between individual students based on cultural knowledge (Alton-Lee, 2003; Andrews \& Yee, 2006) and the likelihood of teachers' dominance over children, both linguistically and through power differentials that are enacted through interactions (Abd-Kadir \& Hardman, 2007; Cazden, 2001; Coles, 2005; Myhill, 2002). In contrast, there are mixed findings in the literature about educating the whole group on the mat; for instance, some studies posit that teachers' believe its use enables children to develop shared interests (Eirich, 2006; Lown, 2002) and group cohesion (Gibbs \& Wilks, 1991; Moss \& Wilson, 1998). On the other hand, other studies assert that it is a highly controlling form of teaching (Leach \& Lewis, 2012; Rubenstein Reich, 1994) that advantages academically high-performing children over others (Piters, 1995).

There are still many gaps in our understanding about children's participation and interactions with peers at mat time. Notwithstanding, children's social lives intersect with teachers' pedagogy and ideology. The official ways of being within classroom life, which are often sanctioned by the teacher (Woodrow, 2006), impact on how children are able to relate to each other. The resulting milieu is characterised by both overt scripts and hidden power relations. It is in the concept of this intersecting place that the present research is situated.

\subsection{Teachers' and children's perspectives of the mat}

In order to understand a phenomenon, it is useful to be cognisant of the perspectives of the people who have lived experience of that phenomenon; however, there is a paucity of reporting from children's perspectives about the 
mat, which means that little is known about what children think about it, and whether this has any bearing on their learning. Studies that have investigated children's views have generally been small-scale studies that have focused on a single aspect. Examples include Wood et al.'s (2009) study that suggested that five and six year old children liked the use of response cards, and Cefai at al.'s (2013) report that children preferred lively activities such as games and singing but were concerned about disruptive behaviours. Other studies have found that some young children thought that mat time was for learning rules such as sitting still (Rubenstein Reich, 1994), and that when there was an emphasis at mat time on children's self-esteem and group cohesion, children mostly agreed that the mat times improved the social climate of the classroom (Lown, 2002; Moss \& Wilson, 1998).

There are more studies that report teachers' perspectives; for instance, teachers in three studies described their teaching practices on the mat as predominantly being responsive to children because they incorporated children's interests (Eirich, 2006; Gibbs \& Wilks, 1991; Hong, 1995). Mat time sometimes appears to have a focus on instruction, which might impede a teacher's ability to respond to children's interests if those interests differ to the planned topic of instruction. Gibbs and Wilks, and Hong, indicated that teachers of three and four year old children reported that mat time was useful for learning academic concepts. Nonetheless, few mat time studies have reported that teachers hold concerns about tensions between instruction and responding to children's interests. Hong reported that a teacher has to balance her lesson plans with the ideas that children raised in discussion. However, it did not seem that this facet caused her concern. In fact, the teacher reported that she enjoyed mat time, and that facilitating it suited her personality traits as dominant and creative. As such, the teacher experienced personal gratification from facilitating activity on the mat.

Nevertheless, there are hints in the literature that mat time might entail considerable challenges for teachers and children. When reporting on her own 
class, Eirich (2006) was anxious that children should use desired communicative behaviours (such as not interrupting others when speaking). Gallas (1992) was concerned that children sometimes disclose personal information at mat time, and that this provides potential for emotional detriment if other class members choose to relay the information to others or to use it as a basis for taunts. For instance, at one mat time, a child told the class about her father's cocaine addiction and incarceration. In addition, Rubenstein Reich (1994) identified a list of pedagogical tensions that teachers struggle with, such as balancing the needs of individual children with those of the group.

Such concerns might be resolved through the use of partnership approaches between teachers and children (Johnston et al., 2002). The teacher in the studies by Danielewicz et al. (1996) and Gallas (1992) indicated that she believed it to be important to hand a certain degree of control over the mat to the children. As such, children were enabled to influence the content and the structure of the mat times. Indeed, Eirich (2006) suggested that a useful pedagogical approach at mat time is one that is democratic, whereby control is distributed across the group through voting. Nevertheless, other studies (for instance, Piters, 1995) found that the teacher strictly controlled the interactions at mat time and did not appear to use practices that were as responsive to children as those described in the previous studies. This view was consistent with Danielewicz et al. (1996), who described general teacher practices, other than those of their study-participant, as being highly controlling.

Teachers participating in several studies reported mat time as fostering a sense of togetherness or social cohesion among groups of children (Eirich, 2006; Gibbs \& Wilks, 1991; Hong, 1995). In contrast to the circle time studies, which reveal a deliberate focus on togetherness, other mat time literature is not clear on whether a sense of community is typically achieved. However, Eirich (2006) described how she facilitated a contract with her class of six to eight year olds, about rules for interacting with one other. A tentative case could be made that enforcing the 
contract might have contributed to a sense of togetherness, in that she emphasised prosocial traits such as listening to speakers and acting in fairness. Opportunities for children to share their interests with each other were inferred as promoting a sense of togetherness by Danielewicz et al. (1996) and Gallas (1992), who each researched sharing time with six to seven year olds.

Notwithstanding, these reports were based on teachers' perceptions, rather than any empirical evidence of togetherness.

A sense of togetherness and cohesion is implied in Eggen and Kauchak's (2006) assertion that the social climate in the classroom should be safe. However, they also state that the climate should be orderly, and that teachers should aim to engage children's attentiveness. It can be assumed that the development of such a social climate requires more than just the social contract described by Eirich (2006). Indeed, she also reported ignoring interruptions and acknowledging specific children's attempts to adhere to the rules about behaviour on the mat. In contrast, Gibbs and Wilks (1991) reported that the teachers in their study placed little emphasis on children's compliance with rules. Further investigation about the social climate on the mat is warranted, especially in respect of its potential impact on children's participation.

It should also be noted that even though researchers made frequent references to togetherness or group cohesion, these concepts were infrequently defined (for example, see Gibbs \& Wilks, 1991; Lown, 2002; Moss \& Wilson, 1998). The present study uses the conceptualisation of group cohesion by Treadwell, Reisch, Travaglini, and Kumar (2011), who liken it to the development of "healthy interpersonal attachments and instilling a sense of togetherness" (p. 504). Furthermore, they assert that it is a critical factor for establishing a safe emotional climate for group members. Togetherness is typically described as the effect of shared care, understandings, practices, and values, among individuals within a group, that are used to develop and maintain social bonds between them (Avgitidou , 2001; De Haan \& Singer, 2001; Van Oers \& Hännikäinen 2001). 


\subsection{Participation}

All of the studies included in the present review refer to children 'doing things'; however, little attention has been given to children's active participation, as opposed to their observing, during activities on the mat. Nevertheless, two studies highlight the importance of active participation on the mat to children's learning (i.e., Han et al., 2005; Mandel Morrow \& Smith, 1990). Video observations taken in North American kindergarten classrooms compared two curricula, one that allowed many opportunities for children to speak on the mat, and another that required them to listen more. The curriculum that invited children's active participation to a greater extent was positively associated with increases in their language development (Han et al., 2005). Similarly, Mandel Morrow and Smith (1990) assessed children's comprehension after reading books one-to-one, in small groups, and large groups. Comprehension was more sophisticated in the small group scenario, possibly because children could share ideas with each other, which they were unable to do in the one-to-one scenario, and could do in the small group more easily than in the large group. Moreover, the further away from the teacher that children sat, the more difficult it was for them to actively participate.

The fact that Mandel Morrow and Smith (1990) found that children who sat at the back during book reading sessions found it difficult to participate in discussion suggests that gaining a participatory role on the mat is potentially competitive. The mechanics of such competition are illustrated well by Putallaz and Sheppard (1992) who argue that dominant children are able to meet their own agenda by being socially perceptive about the setting and the other group members in order to utilise the most effective strategies at the most effective times. One of the key issues here is that teachers need to be aware of relationships between their practices and the peer group (Green \& Rechis, 2006).

A second study by Piters (1995) investigated participation in a single classroom. She found that the teacher invited specific children to participate more than others. Such children were predominantly high achieving. Moreover, their 
participation subsequently attracted praise and feedback from the teacher. Given that teachers' approval has been associated with children's reputation among the peer group (see El-Ghoroury, 2002; Mikami et al., 2012; White et al., 1998; White \& Kistner, 1992), the praise may have served to enhance a child's reputation among peers. As such, participation might be an important factor in the social lives of children.

A focus on participation is important, given that a child's developing self-concept is influenced by the social and physical contexts within which they participate, as well as the apparent equity of these contexts (Hughes \& Zhang, 2007). However, different people experience a given environment in a multitude of ways; therefore, that environment will influence them in varying ways. An individual's participation in the social activities within any environment is not merely based on their individual involvement-as-doing, but on their interactions and communication with others. In turn, an individual's participation with others partway informs their sense of belonging and sense of self (Sandberg \& Eriksson, 2008). In other words, a person's self-concept is influenced by the ways in which they participate with others in the environment. Relevant factors include the sense of personal agency that is afforded to an individual in the social environment, the types of roles that an individual can undertake, and the quality of their personal relationships and interactions. With regard to the latter, Bukowski et al. (2011) assert that:

People define themselves according to how they believe they are perceived by others...one's recognition of how one is perceived and treated by others forms the basis not only of the self-concept but also of how one perceives others. (p. 155)

Sandberg and Eriksson (2008) suggest a threefold conceptualisation of participation, identified as: (1) the ability to influence, (2) possessing a sense of belonging and support and (3) actively taking part in the carrying out of activities. 
This conceptualisation of participation encapsulates the notions of influence and social relations that other authors have raised as important to students' participation (for examples, see Collins, 2013; Leach \& Lewis, 2012). With regard to influence and belonging, Sandberg and Eriksson directly refer to "solidarity, unity, and fellowship" (p. 624). Moreover, by emphasising active participation, Sandberg and Eriksson's conceptualisation is consistent with that of Weaver \& Qi, (2005), who distinguish between contribution, and listening or observing.

\subsection{The interactional milieu of the mat}

A view of participation as contribution, support, and influence (Sandberg \& Eriksson, 2008) directly relates to the ways in which class members interact with each other; in the present research, this is labelled as the interactional milieu. In the first instance, the interactional milieu comprises intentions and decisions of the teacher. These operate within a terrain that Gutierrez and Stone (2000) described as official space. Official space contains scripts that form patterns of discourse and interaction that guide the nature of participation for individuals. The teacher is a strong determining factor in the nature of the official space. Notwithstanding, some students possess familiarity with the official scripts, and willingness to participate and contribute to them; however, other students do not (Gutierrez \& Stone, 2000). Woodrow (2006) defines official space as being:

characterised by official authority; the teacher maintains absolute control over both the course of talk in the classroom and the interactions that will be officially recognised. While some students both contribute to and participate in the teacher's official script, others form their own counter-script. (p. 3)

Similarly, McLaren (1999) refers to the official nature of teachers' voice and asserts that it "reflects the values, ideologies, and structuring principles that teachers use to understand and mediate the histories, cultures, and subjectivities of their students" (p. 245). As such, the official space on the mat informs students 
about which literacies, working theories, dispositions, social roles, and learning strategies are valued in the setting. Another way of putting it is that official space often defaults to the ways of being and knowledge valued by the status quo (see Alton-Lee, 2003; Andrews \& Yee, 2006; Hogg, 2013).

The notion of official space places emphasis on predetermined notions of competence. In order to meet these prescribed notions, teachers use strategies such as discussion or explicit instruction that might be typically utilised on the mat. At such times, specific behaviours are required, such as focusing on a task, and paying attention (see Tominey \& McClelland, 2011; Vargo, 2008; Wood et al., 2009). Another required behaviour might consist of children's delivery of correct responses to their teacher's questions (Piters, 1995). To put it simply, the official space conveys to students that specific academic and social behaviours are desirable to a teacher. Thus a particular view of competence is promoted through classroom discourse; one that is compliant to a teacher's expectations.

Although the official space communicates expectations and demands to students, not all students share the attitudes, characteristics, temperament, or social capital that are congruent with the demands of the official space. Churchill (2003) refers to the degree of congruence as the "goodness-of-fit"2 (p. 113) between a child's social capital and the demands of his or her educational environment. For instance, certain children will enter a classroom already equipped with many social understandings that meet with the teacher's approval. As such, they may have an advantage over their peers who do not, because goodness-of-fit most likely influences the child's reputation, and how well he or she is understood by the teacher. Churchill states that "goodness-of-fit helps us to conceptualise how some teachers and some children are more easily able to establish and maintain [positive] relationships" (p. 116).

2 This term was originally coined by Thomas and Cress (1977) in relation to the degree of attunement between parents and children. 
Official space is often contrasted with unofficial space. Woodrow (2006) uses the latter term to describe the terrain that is antipodal to that of official space. Whereas official space is the province of overt teacher control, unofficial space is characterised by students' discourses that counter those pertaining to official space. In other words, unofficial space is created when students resist their teacher's scripts, or patterned actions (Gutierrez \& Stone, 2000; Woodrow, 2006). Counter-scripts may be evident if a student's cultural needs conflict with those of the teacher. Generally, unofficial space includes children's knowledge and skills that are validated in the home environment or other settings outside of the classroom; however, these knowledge and skills are rendered invisible or nonvalid by the official space (Gutierrez \& Stone, 2000; Woodrow, 2006).

In the present research, the terrain of the students is referred to as the children's social worlds rather than unofficial space. Previous research on unofficial space has focused on children's cultural knowledge and the dissonances caused in relating to the dominant classroom culture (Gutierrez \& Stone, 2000; Woodrow, 2006). The present research is founded on an assumption that children's culture is an essential aspect of their self-concept and impacts on their access to opportunities in the classroom. However, what is proposed here is that children's peer relations might also impact their self-concepts and access to opportunities. As such, children's social worlds form a second focus alongside the official space. A deliberate choice was made to refer to social worlds in plural, because it is likely that children operate in several sub-groups within a single classroom; each subgroup most likely has its own behavioural norms and values (Galbraith, 2011).

The concept of children's social worlds and that of unofficial space are similar, in that studies about children's peer relations (see Corsaro, 1988, 2003; Eirich, 2006) and unofficial space (see Guitierrez \& Stone, 2000; Woodrow, 2006) assert that the worlds of adults and children overlap to create a shared, middle space (see Figure 3.1). It is in this intersecting space that the present research is situated, and what is meant when I refer to the interactional milieu of the mat. 
This space attempts to link the curriculum, and values of the official space, with the children's lived social experiences, understandings, agendas, roles, strategies, and skills contained in their social worlds.

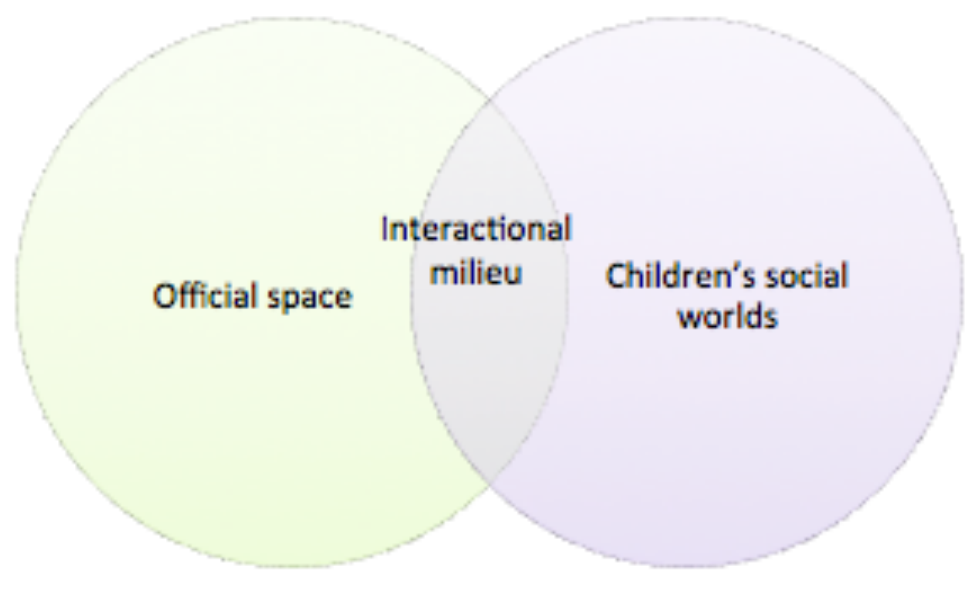

Figure 3.1 The interactional milieu as an intersection between the official space and children's social worlds

Figure 3.1 depicts a shared interactional milieu between the official space and children's social worlds. The interactional milieu comprises a symbiotic relationship between teachers' pedagogy ${ }^{3}$ and the interests and needs contained within the children's social worlds. The effects of the interactional milieu can result in a kind of en dehors ${ }^{4}$, in that certain interests and needs are prioritised more than others. One example is Eirich's (2006) strategy of asking the children in her class how they wanted to choose which children spoke at morning meeting. This strategy was consistent with the discourses pertaining to democracy that were espoused in the official space of her classroom. Rees (a child) responded,

3 The specific theoretical underpinnings of pedagogy and children's social worlds were presented in the preceding two chapters.

${ }^{4}$ En dehors refers to a musical direction that makes a specific melody stand out as well as being French for outside. In the present research, en dehors refers to practices and interactions that simultaneously privilege the interests of some children (making them stand out) while disadvantaging others (relegating them to outside of the experience). 
"we could, like, vote to, like, decide which people share" (p. 179). While democratic strategies can be seen to empower the children as a whole group, a democratic approach would also allow votes that favour certain children over others, thus entitling them to a more active participatory role. In this example, en dehors describes an outcome that favours popular children whilst simultaneously disadvantaging their less popular peers. Either way, there are direct implications for children's interactions and opportunities for participation.

\subsection{New Zealand primary classrooms}

The interactional milieu is further influenced by the physical context in which it is situated. The present research was undertaken in New Zealand primary schools. Children customarily start school in New Zealand on their fifth birthday. Schools are funded by the Ministry of Education, and are governed by boards of trustees constituting school staff and parents. Classes typically comprise approximately 20 to 30 children and one teacher. In addition, many classes have a teacher aide to assist in the provision of inclusive education for students with disabilities. New Zealand classrooms are largely similar to those in other countries, in that they generally contain tables, chairs, and a carpeted area, known as the mat. Children attend school for six hours a day and lessons are punctuated with breaks. Teachers assess individual learning, which is achieved, in part, through teachers' observations of children's participation and performance in various activities and tasks.

The New Zealand Curriculum Framework (NZCF) guides pedagogy in New Zealand classrooms (Ministry of Education, 2007). It synergises social constructivist principles of learning, with prescribed levels of skills and understanding that students are expected to achieve in particular subject domains. At the outset, subject domain areas that are seen to be essential for today's student are listed, such as English, The Arts, Mathematics, and Technology. The subject domains sit alongside key competencies, for instance, relating to others, participating and contributing. In addition, values are espoused (such as inquiry and curiosity, 
equity, community, and participation). Moreover, the subject domains, key competencies, and values are all underpinned by social principles, such as cultural diversity and inclusion (Ministry of Education, 2007). A section of the NZCF, entitled 'Effective Pedagogy', situates the student as being central to pedagogy:

Learning is inseparable from its social and cultural context.

Students learn best when they feel accepted, when they enjoy positive relationships with their fellow students and teachers, when they are able to be active, visible members of the learning community. (Ministry of Education, 2007, p. 34)

This aligns pedagogical expectations with the view that affirming relationships and social safety are integral to learning. Nonetheless, despite the central positioning of the student, the curriculum prescribes levels and outcomes that teachers and students are required to focus on.

\subsection{Statement of the problem and purpose of the study}

In recent years, several key pedagogical texts have associated young children's positive and active participation in classroom learning activities with the quality of their learning, sociability, and self-concepts (for example, see Farmer, McAuliffe, \& Hamm, 2011; McGee \& Fraser, 2012). However, observational research indicates that individual children have varying involvement in groupactivities, despite the presence of the teacher (Myhill, 2002; Piters, 1995). This suggests that there may be complex reasons for the differences in young children's active participation in whole-class activities, and that these might be partially hidden from the adult-view. The present research investigates teachers' and children's perspectives about one whole-class activity, namely mat time.

Use of the mat has been a little-researched phenomenon in New Zealand; therefore, it is worthwhile investigating it further in order to understand the interactional milieu that it affords. Several international studies suggested that 
there might be tensions between teachers and children during mat-based activity, particularly with regard to children's attentiveness and behaviour (Ling \& Barnett, 2013; Vargo, 2008; Zaghlawan \& Ostrosky, 2011). It is likely that an investigation of the perspectives of teachers and children may provide insights into ways to mitigate such tensions, especially if there is a disconnect between teachers' views and those of children. Conversely, because so little is known about use of the mat in New Zealand, it is uncertain which aspects of practice work well for teachers and children. The purpose of the present research is to understand how teachers and children perceive official space pertaining to the mat.

Furthermore, a second purpose is to investigate the nature of children's social worlds at mat time and the roles of those social worlds in relation to children's involvement and participation.

\subsection{Epistemology}

Crotty (1998) suggested that research is guided by three key epistemologies: constructionism, objectivity, and subjectivity. The present thesis is influenced by constructionism, specifically the notion that reality is socially constructed. One underlying philosophy arising from constructionism, and that is relevant to this thesis, is pragmatism. Johnson and Ongwuebuzie (2004) describe a pragmatic approach as one that views knowledge as both socially constructed and based on

the reality of the world that human beings live in. In fact, truth and knowledge are viewed as fluid constructs that change over time. In addition, pragmatism is strongly oriented toward equality and the idea that effective practice in any arena must be informed by theory (Johnson \& Ongwuebuzie, 2004).

\subsection{The research questions and methods}

Children's participation matters, given that it is associated with learning (Hidi \& Renninger, 2006; Tominey \& McClelland, 2011) and self-concepts (Skinner et al., 1990); however, the official space of the classroom influences children's involvement. The most obvious example includes teachers' explicit pedagogical decisions about the structure or activity. A compelling example was given by 
Wood et al. (2009) when the teacher moved away from calling on one or two children to respond to questions, and instead used response cards; this meant that the whole class could participate simultaneously. Other examples given included the use of group games that enabled each child's involvement (Mary, 2014; Svinth, 2013), or relaxing some of the teacher-control, allowing children to interrupt each other (Murphy, 2003).

In addition to pedagogical decisions, it seems likely that there are other, more hidden, factors that influence the interactional milieu. For example, Danielewicz et al. (1996) reported that a peer group looked disinterested when specific children spoke, but supported the contributions of popular peers. Similarly, Cefai et al. (2014) suggested that some children use information shared by children at mat time to discredit them. This might lead to specific children withdrawing their participation in order to protect themselves from the relational aggression of peers.

Two research questions were developed in order to investigate the pedagogical and social aspects of mat time. In addition, the perspectives of both teachers and children were considered in order to more fully understand the phenomenon. The research questions were:

- How do teachers' and children's perspectives of mat time compare?

- How does the interactional milieu influence children's involvement at mat time?

In order to answer the research questions, two studies are reported. These studies followed a mixed method sequential explanatory design (as described by Ivankova, Cresswell, \& Stick, 2006). The first study was a national survey of teachers in New Zealand year two classrooms. In addition to the overarching 
research questions, several sub-questions were developed to focus data collection. These were:

- How much of the child's school day is spent on the mat in junior classrooms?

- What does mat time contribute to children's learning, according to New Zealand primary school teachers?

- What pedagogies do teachers associate with the use of the mat in junior classrooms?

- How do teachers view young children's participation and engagement at mat time?

- What characteristics comprise the official space of mat time?

The study focused on teachers' reports about their pedagogy and their perceptions about use of the mat. Furthermore, they were asked about children's participation. Finally, teachers were asked to indicate their willingness to have their class participate in in-depth research, and three classes were purposively chosen for this second study.

The second study used semi-structured interviews with children. Interviews used small props and asked children to use them to explain what happens on the mat. They were also asked to indicate their feelings about the mat and explain whether their personal participation was important. The sub-questions addressed by the second study were:

- What are children's impressions of mat time?

- How important do children consider their own participation and engagement to be at mat time?

- How do children's peer relations influence their participation at mat time?

Classroom video-footage and semi-structured interviews with the three classroom teachers were undertaken. These were used to validate the accuracy of 
my assumptions. As such, the data arising from the video recordings and teacherinterviews are not reported on in this thesis, except for when they provide an illustration that clarifies the findings arising from the children's interviews. An additional point is that the video-recordings and teacher-interviews generated a significant amount of data. These data are too numerous to be easily managed in a single thesis and will form the basis of subsequent publications.

\subsection{The researcher's background}

I have been involved in early childhood education since 1996, first as a teacher, and then as a professional development facilitator and lecturer in initial teacher education programmes. In 2005 I completed research for a Masters Degree that investigated very young children's problem-solving skills within a technology education framework. At the same time, I was one of the researchers on a Ministry of Education funded project, which later underpinned the publication Kei Tua o te Pae, Assessment for Learning in Early Childhood (Ministry of Education, 2004). One of the things that was noticeable in each of these roles was that certain children were highly visible within educational settings, whereas others were comparatively hidden. This observation informed the theme for a series of teacher professional development sessions, in which the teacher-participants largely agreed that specific children were more noticeable to them compared to others. Nevertheless, teachers seemed perplexed as to why this should be. These experiences fostered my desire to understand more about young children's roles and their impact on their participation in educational activities.

\subsection{Chapter summary and conclusions}

The studies that report teachers' perspectives about mat time indicate that teachers appear to view it as something that engenders many positive outcomes for children, and that it is enjoyable for children (for examples, see Danielewicz et al., 1996; Eirich, 2006; Gallas, 1992; Gibbs \& Wilks, 1991; Hong, 1995; Rubenstein Reich, 1994). In contrast, there are few studies that have sought children's perspectives, and those that have, have focused on such a diverse array of topics, 
that it is difficult to make any general inferences about how children view their mat time experiences in particular. Nevertheless, there is a small amount of evidence that peer issues might impact on children's experience of mat time; for example, Cefai et al. (2013) found that many children were bothered by the behaviour of their peers on the mat, yet this did not seem to be a problematic issue for teachers.

There is merit in exploring how the views of teachers and children intersect, in order to understand teaching and learning on the mat. The concept of an interactional milieu is a useful one. In short, the interactional milieu of the mat is influenced by official space, and comprises teacher-authority, curriculum, and ideology. This interactional milieu is also influenced by children's social worlds, characterised by their peer culture, peer relations, and internal hierarchy.

Overall, the interactional milieu will most likely impact on a range of opportunities for children's participation on the mat, and vice versa, but very little is known about it. Notwithstanding, there is a vast array of studies that have examined children's participation in general classroom situations and that have indicated that at least some of the disparities in children's active involvement are attributable to teachers' pedagogy (see Alton-Lee, 2003; El-Ghoroury, 2002; Mikami et al., 2012; Myhill et al., 2006). In addition, Sandberg and Eriksson (2011) suggest that individual children's participation might be impacted by the degree of influence and social support that they possess. However, there has been very little focus in the mat time literature on the enablers and barriers that children experience with regard to participation; therefore, this is a focus of the present research. 


\section{CHAPTER FOUR \\ Study One}

\subsection{Introduction}

This chapter reports on the first of two studies that examined participants' perspectives on mat time. Study One, comprising a quantitative analysis of a largescale survey, investigated teachers' perspectives. The aim was to investigate the official space of the mat, and understand the pedagogies associated with it. A teacher must respond to many facets of each student, including self-concept, goals, working theories, and interests. It follows that teaching multiple students simultaneously is highly complex and most likely presents teachers with a range

of pedagogical tensions. Investigating those pedagogical and social complexities at mat time is imperative in order to understand the influence that teachers' strategies have on the interactional milieu of mat time.

The international literature showed that mat time predominantly was arranged along three main foci: instructional (Björk-Willén, 2008; Duman, 2009; Ernst, 1994; Han et al., 2005; Hong, 1995), sharing time (Danielewicz et al., 1996; Gallas, 1992; Murphy, 2003), or more playful activities, such as music and games (Mary, 2012; Svinth, 2013). Each of these types of activities would require different degrees of teacher facilitation and would enable a range of interactional opportunities.

Teachers intend a wide range of pedagogical goals, arising from these mat time activities; for instance, fostering children's enhanced communication skills (Eirich, 2006; Ernst, 1994), self-esteem, group cohesion (Canney \& Byrne, 2006; Collins, 2013), and academic skills (Duman, 2009; Han et al., 2005). There is currently no information about New Zealand teachers' pedagogical goals for mat time. Nevertheless, developing a better understanding of what teachers report that they achieve at mat time part-way contextualises the pedagogy. For example, if teachers agree that mat time achieves group cohesion, then it could be expected that their choice of activities or ratings of children's behaviour would reflect that. 
It is possible that aligning activities with many pedagogical goals might present significant pedagogical tensions or challenges for teachers. Rubenstein Reich (1994) argued that Swedish preschool teachers attempt to achieve too many outcomes at mat time, and that some of them are incompatible with each other, creating tensions for teachers. Moreover, these tensions impact on interactions with children. Gallas (1992) and Poveda (2001) expressed a similar sentiment with regard to teachers' social and academic goals for children's narratives. Given that New Zealand teachers are tasked with synergising social constructivist principles of pedagogy with the predetermined outcomes laid out in the NZCF (Ministry of Education, 2007), it seems that they too, might experience pedagogical dissonances. Nonetheless, so little is known empirically about mat time pedagogy in New Zealand, that assumptions about pedagogical, or even ideological, difficulties cannot be made with any validity.

Another consideration for teachers might be how to attract children's attentiveness. Milman (2009) posits that attentiveness is often associated with on-task behaviour. Pedagogical strategies aimed to catch children's on-task behaviour can range from teachers taking control to attempting to motivate children to participate. In either case, strategies that teachers use for engaging children's attention at mat time relate directly to the scripts pertaining to the official space of the mat; they communicate the power-roles inherent in what it means to be a teacher or a student. Moreover, those strategies, used to elicit children's active involvement, impact on the social climate; for example, reprimands might set a more negative tone than say, making mat time interesting and engaging. In either case, it is currently unclear what strategies New Zealand teachers use to elicit children's engagement in mat time tasks, or what impact these strategies have on the interactional milieu.

The international literature suggests that interactions on the mat can be fraught if children behave in challenging ways. Previous studies have found that specific 
children were disruptive, or dominating (Ling \& Barnett, 2013; Vargo, 2008; Zaghlawan \& Ostrosky, 2011). In addition, Rubenstein Reich (1994) found that certain children withdrew from active participation, or used off-task behaviours, as a form of protest against teacher control. Nevertheless, each of these studies was undertaken with younger children; therefore, it is unknown whether these behaviours were predominantly attributable to developmental factors. A further point is that children's challenging behaviour might be associated with their enjoyment of mat time. The international literature presented conflicting findings in this regard (for example, see Lown, 2002; Leach \& Lewis, 2012). Investigation into New Zealand teachers' beliefs about children's enjoyment of mat time is warranted.

These challenging behaviours would have a strong impact on the type of active involvement that children had at mat time. Another influence on children's participation and interactions is the pedagogical approach that teachers predominantly use. The teachers' general approach at mat time informs the interactional milieu of the mat; for example, child-directedness might allow for a more discursive style of interaction (Danielewicz et al., 1996; Gallas, 1992; Murphy, 2003). Either way, the extant literature has little information about the likelihood of every child contributing at mat time, or the ways in which the pedagogical approaches enable or deter each child's involvement.

\subsection{Study aims and research questions}

In order to address the main research questions it was important to gain an understanding of mat time, as a phenomenon, in New Zealand classrooms. The aim of the present study was to gather teachers' views about pedagogy using the mat, as well as their views of children's participation. The intent was to understand the official space, as described by Woodrow (2006), of the mat. In other words, the survey analysis was concerned with the way in which mat time pedagogy informs and validates specific ideology, ways of being, power, participation, and interactional patterns. A specific question was used to focus the research: 
- What characteristics comprise the official space of mat time?

In addition, several other questions were used to frame the data gathering:

- How much of the child's school day is spent on the mat in junior classrooms?

- What does mat time contribute to children's learning, according to New Zealand primary school teachers?

- What pedagogies do teachers associate with the use of the mat in junior classrooms?

- Are there any tensions between these pedagogies?

- How do teachers view young children's participation and engagement at mat time?

- How do they optimise participation and engagement?

\subsection{Method}

A questionnaire was used to elicit teachers' self-reports about their pedagogy and their perceptions of children's behaviour at mat time. The use of a survey is beneficial when the perspectives of a large number of people are required and when insights into their attitudes, behaviours, and perceptions are needed. This is because a single researcher can manage data gathering more easily than interview methods, but it still allows for respondents to disclose relevant information. In addition, the use of questionnaire is more likely, than many other methods, to result in generalisable findings, provided the sample is representative of the population being investigated (Jenkins, 1999; Johnson \& Christensen, 2008).

\subsubsection{Sampling}

Fluctuating membership of peer groups can create conflict and social upheaval (McGrew, 1972). It follows that some children's social behaviour is likely to be affected by changes to group membership, particularly those pertaining to social 
hierarchies and peer relations. Therefore, it was important to select a population on which to focus the present study that would be minimally impacted by incoming and outgoing group members. In the New Zealand school system, year two is the first year in which the group of children in a classroom is typically stable (i.e., without individual or group entry based on children's fifth birthdays). As such, teachers of year two classrooms in New Zealand were invited to participate in the current research via an email sent to the school, using the Ministry of Education directory. No definitive data on the total numbers of teachers in year two classrooms in 2011 were available; however, a spokesperson in the Educational Information and Analysis section of the Ministry of Education gave an approximate figure of 2,500 teachers. The sample comprised teachers who accepted the invitation.

\subsubsection{Design of the survey Instrument}

The survey is presented in full in Appendix A. The online site Qualtrics was used to present the survey to respondents and to gather the data. The items for the survey were developed following reviews of relevant literature about mat time and discussions with six primary school teachers within my networks, and who acted as critical peers. Before dissemination, the survey was taken to Victoria University's statistical consultant for a final review.

The survey consisted of categorical questions, Likert scale questions, and other descriptive rating scales. In addition, qualitative questions were asked and opportunities for respondents to make further comments were given. Overall, there were 26 questions. Six pertained to respondents' demographics and schools. Eight questions asked about mat time. Nine questions offered respondents the opportunity to qualify their responses or make other comments. The final three questions asked respondents to indicate if they wished to: receive a brief report of survey findings, participate in Study Two, and participate in the incentive-draw. 


\section{Categorical questions}

First, the survey asked respondents to give information about themselves, asking respondents to indicate their gender, and then choose from six options that best indicated the number of years they have been teaching '1-2', '3-5' through to 'more than 20').

Information about the respondents' schools was sought. Four options describing types of school were presented ('state', 'private', 'Māori immersion', and 'affiliated to a religion'), and respondents were asked to choose those which best applied. Various school locations were given ('inner city', 'in a suburban area', and 'rural') and respondents were asked to select which one best described their context. In addition they were asked to indicate their school's decile rating (from 1-10).

The amount of time that children spend on the mat was investigated through two questions. The first asked respondents to choose from 5 options, describing average number of mat times held in any given day ('1-3', '4-6', through to '13 or more'). The second question presented 6 options that described the average length of mat times ('less than 10 minutes', '10-20 minutes', through to 'longer than 50 minutes').

A drop-down list of 7 strategies for engaging children's attention was given, which included those strategies that use either positive or negative behavioural reinforcement (issuing reprimands, offering rewards, writing children's names on the board, seating specific children close to the teacher, and removing disruptive children form the mat) and those that aim to encourage students' motivation (incorporating students' interests and using interesting voice intonation). Respondents were asked to select all of those strategies that they had used. 


\section{Likert scales}

In order to gather information about teachers' beliefs about mat time, respondents were asked to indicate their agreement on two series of five-point Likert scales, ranging from strongly disagree to strongly agree. In total, there were 15 Likert scales.

The first series of scales asked respondents to rate their agreement about the efficacy of mat time for achieving nine pedagogical goals. They were asked to what extent they agreed that mat time contributed to group cohesion, children's selfesteem, and the provision of a sense of rhythm for the day. Other scales in this series asked about respondents' agreement that mat time contributed to the development of children's cognitive knowledge, language, listening skills, and motor skills. The final scale of this series asked about respondents' agreement that mat time assisted children to develop restraint and self-control.

A second series of six Likert scales asked teachers to indicate their agreement to statements about children's behaviour at mat time. The items included: 'particular children tend to dominate', 'particular children observe only', 'children are attentive', 'particular children always seem to put their hand up', 'particular children are consistently disruptive', and 'children enjoy mat time'.

\section{Other descriptive rating scales}

In addition to the Likert three other scales were used; however, these additional scales asked respondents to report on their practices, rather than their beliefs. The first scale consisted of 13 activities for mat time and asked respondents to indicate how often mat time was used for each activity. Some of the activities included reading a book, teaching curriculum knowledge, and music. The scale had five points consisting of 'never', 'only sometimes, not every day', 'every day', '2-3 times a day', and ' 3 or more times a day'. 
The second scale explored the degree of challenge that respondents experienced in relation to five tensions and challenges. The first two tensions were 'balancing the needs of individual children against the needs of the group', and 'delivering the curriculum versus following children's interests'. The pedagogical challenges included 'ensuring that all children who want to have a turn to contribute or participate', 'managing non-compliant behaviour', and 'maintaining all children's engagement'. The respondents were asked to indicate how challenging they found each of these issues to resolve by using a five-point scale ranging from 'extremely challenging' to 'extremely easy'. The midpoint on the scale was 'sometimes challenging and sometimes easy'.

Respondents were also asked to place their mat time pedagogy on five-point rating scales for a range of five practices arranged between two opposing poles. These included 'highly teacher-directed' to 'highly child-directed', 'informal to formal', 'instructive to discussive', and 'unplanned or spontaneous' to 'planned'. A final scale comprised 'low frequency of questions asked of children' to 'high frequency of questions asked of children'.

A final, stand-alone scale was presented that asked respondents to indicate the likelihood that all children participate at mat time. The scale comprised of five points ranging from 'very unlikely' to 'very likely', with 'undecided' as the midpoint on the scale.

\section{Qualitative questions}

Respondents were asked to nominated further options, other than those given in the scales for: uses of mat time, pedagogical challenges and tensions, strategies for engaging children's attention, children's behaviour at mat time, and teaching strategies. They were also asked if they wanted to say more about children's contribution or participation at mat time. Two further questions asked about the benefits for children and teachers. Finally, respondents were invited to share any further comment about mat time. 


\subsubsection{Pilot testing}

Before wider dissemination, the survey was pilot tested and amendments were made, as suggested by Jenkins (1999). Twelve volunteers who were junior primary school teachers were sourced through my networks. Relevant changes were made to the survey based on their feedback. For example, in the pilot survey, removing children from the mat was referred to as exclusion. However, feedback indicated that the teachers use this term to refer to situations in which children face expulsion or suspension. Therefore, exclusion was amended to removing children from the mat. Another example was their preference for the word discussive to denote a style of facilitation that utilised group discussion, rather than the more grammatically correct use of discursive.

\subsubsection{Administration of the survey}

After ethical approval was granted by the Victoria University of Wellington Faculty of Education Human Ethics Committee (ref number: SEPP/2010/115 RM18188, see Appendix B), email addresses for schools were downloaded from the Ministry of Education website. An email was sent to 1,914 schools between the $7^{\text {th }}$ and $27^{\text {th }}$ of June 2011 containing a URL link to the survey on Qualtrics and a request that the email be forwarded to relevant staff. An information letter describing the research aims, ethical considerations and use of responses was attached. Schools from all regions of New Zealand were included, excepting Canterbury, owing to the devastating effects of large earthquakes.

\subsubsection{Data Analysis strategies}

Proportions of responses in each category and their associated standard errors were plotted on histograms. Principal component analyses were undertaken in order to understand the dimensionality of the data, and as a preliminary to calibrating measurement variables. Use of the principal component analyses enabled identification of items in relation to associated traits. The Kaiser (1970, cited in Osborne, Costello, \& Kellow, 2008) criterion was used, meaning that factors were retained if they were associated with eigenvalues greater than 1 . 
Varimax orthogonal rotation was employed to maximise the variances of factors (as described by Osborne et al., 2008). Scales were calibrated for the groups of items associated with each factor using Rasch modelling, including both dichotomous items; for example, respondents were asked to indicate whether or not they used mat time for a particular activity, and polytomous items; for example, respondents were asked to place their level of agreement with certain statements on a Likert scale. Thus the Rasch modeling located respondents on scales reflecting specific relevant traits (Eggen \& Verhelst, 2011). In other words, these scales were calibrated using Rasch analysis and they effectively aggregated groups of related questions.

Chi-tests were conducted to explore differences in pedagogical approach and children's participation between the teachers who reported using children's interests and those who did not, on discrete (categorical) data variables. This enabled a determination of which differences were statistically significant with the alpha level of significance set at 0.05 (as recommended by Vogt \& Johnson, 2005). Measurements of respondents' general pedagogical approach involved scales ranging from one approach to another arranged on continua; for example, "planned to unplanned". The final question about participation asked teachers to indicate the likelihood that all children participate at mat time on a five-point scale ranging from very unlikely to very likely.

$t$-Tests were conducted to compare differences between teachers who reported utilising children's interests at mat time and teachers who did not on measurement variables; for example, $t$-Tests were used to investigate whether teachers who used children's interests indicated differences in practices to teachers who did not, in respect of 3 factors: possible tensions at mat time, ratings of observable behaviour, and ratings of positive feelings. This entailed a 2-tailed test for equality of means (Vogt \& Johnson, 2005). Specifically, the use of interests was the key variable in each calculation and included two pedagogical practices: the reported inclusion of children's interests at mat time, and teachers' reported 
use of interesting voice intonation. The first factor used for comparison, described possible tensions, and included: maintaining all children's engagement, ensuring the participation of all children, balancing individual and group needs, managing non-compliance, and balancing the curriculum with children's interests. The second and third factors arose from scales that asked respondents to rate their agreement to statements made about children's behaviours and feelings. The second factor (observable behaviour) in the $t$-test was the first principal component arising from the scales, and included: some children dominate, some children observe only, some children always put their hands up, and some children are consistently disruptive. The third factor in the $t$-test used the second principal component from the same scales, and included ratings for children's attentiveness and enjoyment.

Analyses of variance (ANOVAs) were used to test the statistical significance of the differences between the respondents' ratings of children's observable behaviours at mat time (teachers' agreement or disagreement that children consistently behave in varying ways, such as observing or dominating) and agreement on the likelihood that all children participate at mat time or not, using a 2 x 2 design. A second $2 \times 2$ ANOVA assessed the variance between respondents' ratings of children's positive feelings at mat time (children enjoy mat time and are attentive) and the likelihood that all children participate at mat time or not. Finally, the correlations were examined between respondents' ratings of the likelihood that all children participate, and their teaching approaches. These teaching approaches were determined by teachers' placement of their practices on a continuum comprising 'teacher-directed' to 'child-directed'. Spearman's rho was used to compute correlations of pairs of discrete variables (see Vogt \& Johnston, 2005).

\subsection{Results}

\subsubsection{Response rate and demographics of respondents}

Responses to the survey were made by 425 teachers; however, only mostly completed surveys were included in the analysis $(n=296)$. This resulted in the 
analysis of responses from approximately $11.8 \%$ of the target population. There is a possibility of sample bias; teachers who chose to respond might have had a greater propensity to particular perspectives than teachers who chose to not respond.

Table 4.1. Distribution of the Demographic Characteristics of the Sample and the Characteristics of Respondents' Schools

\begin{tabular}{llrr}
\hline Variable & Group & Number & \multicolumn{2}{c}{ Percentage of } \\
& & 274 & 93 \\
Gender & Female & 22 & 7 \\
& Male & $\mathbf{2 9 6}$ & \\
Total & & & \\
Years teaching & $1-2$ & 29 & 10 \\
& $3-5$ & 47 & 16 \\
& $6-10$ & 62 & 21 \\
& $11-15$ & 50 & 17 \\
& $16-20$ & 36 & 12 \\
Total & $>20$ & 71 & 24 \\
& & 295 & \\
Type of school & State primary & 252 & 86 \\
& Private & 6 & 3 \\
& Mãori immersion & 6 & 2 \\
Total & Affiliated to a religion & 32 & 9 \\
& & $\mathbf{2 9 6}$ & \\
Decile rating & $1-3$ & 70 & 24 \\
& $4-7$ & 113 & 38 \\
Total & $8-10$ & 110 & 38 \\
\hline
\end{tabular}

Table 4.1 depicts the demographic characteristics of the respondents and the characteristics of those respondents' schools and shows that of the 296 teachers who completed most of the survey, $93 \%$ were female and $7 \%$ were male. Thus the survey participants were disproportionately weighted towards female teachers, given that national estimates are approximately $82 \%$ female teachers (Ministry of Education, 2005). Nearly a quarter of the respondents were highly experienced 
teachers with twenty years' experience, or more. The large majority of schools were state primary.

\subsubsection{Analysis of the comments section}

The comments sections of the questionnaire were analysed to ensure that respondents' understandings of the constructs were commensurate with my own. Respondents did not mention activities other than those listed in the survey to any great extent. Twelve respondents commented that they used mat time for karakia or prayer, and 8 said that they used it for encouraging the children to sit and have a snack. Most comments affirmed items that were already included in the questionnaire. Finally, two participants made comments objecting to the use of the term 'mat time', describing it as "too babyish" and being more appropriate to early childhood services. However, these two teachers did not indicate a preferred label.

\subsubsection{Time spent on the mat}

Teachers were asked how often they facilitated mat time and the average length of their mat times. The data in Table 4.2 show that time spent on the mat ranged from less than 10 minutes per day to 240 minutes. Typical respondents indicated that they held 4 to 6 mat times per day of 10-20 minutes in length (38\%). These data suggest that a large number of children spent between 40 and 120 minutes per day on the mat (which represents $15 \%-22 \%$ of the children's classroom time).

Table 4.2. Numbers and Average Lengths of Typical Mat Times as Percentages of All Respondents

Average mat time length, minutes

\begin{tabular}{lrrrrrr}
\cline { 2 - 7 } & $1-3$ & $4-6$ & $7-9$ & $10-12$ & $13+$ & Total \\
\hline$<10$ & 1 & 16 & 11 & 2 & 1 & $\mathbf{3 1}$ \\
$10-20$ & 7 & 39 & 14 & 4 & 0 & $\mathbf{6 4}$ \\
$>20$ & 2 & 2 & 1 & 0 & 0 & $\mathbf{5}$ \\
Total & $\mathbf{1 0}$ & $\mathbf{5 7}$ & $\mathbf{2 6}$ & $\mathbf{6}$ & $\mathbf{1}$ & $\mathbf{1 0 0}$ \\
\hline
\end{tabular}




\subsubsection{Activities at mat time}

Teachers were asked to indicate the frequency with which they used various activities at mat time. They were presented with a scale that included (1) never, (2) only sometimes, (3) every day, (4) 2-3 times per day, (5) more than three times per day. Prior to conducting principal component analysis of the activity items, it was speculated that respondents might delineate into those focused on curriculum and those with a more social emphasis. However, as shown by Table 4.3 , three components emerged from principal component analysis, two of which comprised a mixture of curriculum and social items.

Table 4.3. The Three Principal Components, Depicting Loadings >.3, for the Mat Time Activities Item, Comprising of Respondents' Reports on the Frequency with which they Facilitate Social and Academic Activities at Mat Time

\begin{tabular}{lccr}
\hline Activity & Component & & \\
& 1 & 11 & 10 \\
\% of variance & 31 & & \\
& & & \\
Games & $\mathbf{0 . 7 3 9}$ & & \\
Music & $\mathbf{0 . 7 1}$ & & \\
Discussion social issues & $\mathbf{0 . 7 1}$ & & \\
Discussion rules & $\mathbf{0 . 6 7 8}$ & 0.301 & \\
Classroom mgmt. & $\mathbf{0 . 6 3 2}$ & $\mathbf{0 . 8 2 9}$ & \\
Explaining activity & & $\mathbf{0 . 7 1 3}$ & \\
Transitions & & $\mathbf{0 . 6 6 2}$ & \\
Curriculum knowledge & & $\mathbf{0 . 5 7 8}$ & \\
Acknowledgements & & $\mathbf{0 . 4 9 5}$ & \\
Reading book & & & $\mathbf{0 . 7 4 3}$ \\
Farewell & & & $\mathbf{0 . 7 2 9}$ \\
News & & 0.374 & $\mathbf{0 . 5 4 9}$ \\
Welcome & & & \\
\hline
\end{tabular}

The first principal component had an eigenvalue of 4.07. This was associated with games, discussion of social issues, classroom management, music, and discussion of rules. A commonality between these items is related to the classroom's social climate. For example, games have a positive impact on group cohesion when used 
to promote cooperation (Mary, 2012; Svinth, 2013). Similarly, music has been shown to encourage people to synchronise their actions, thereby enhancing affiliative behaviours that are associated with cohesiveness (Harmon-Jones, 2011). In addition, it was reasonable to expect that discussion of social issues, rules, and classroom management related to a positive social climate by establishing clear parameters for operating together.

The second component had an eigenvalue of 1.49. The activities associated with this factor each concerned teaching the curriculum. For example, acknowledgement of good work might have exemplified that which meets the teacher's aims. Similarly, transitioning between tasks or explaining an activity creates structure for assisting children's achievement of a curriculum aim.

The third component had an eigenvalue of 1.25 and consisted of welcome, news (also known as 'sharing time', or 'show and tell'), and farewell. Component 3 related to social niceties that often follow a highly ritualised form (see Kantor et al., 1989). For example, when a teacher calls the roll when welcoming the children, she or he might call the children's names, and the children might respond using a singsong voice each time. The sharing of news also relates to social rituals. For instance, the teacher encourages conversational 'manners', such as listening or speaking clearly; however, many aspects of this activity are also heavily ritualised because there are set rules governing how it happens (Danielewics et al., 1996). 
1. Games

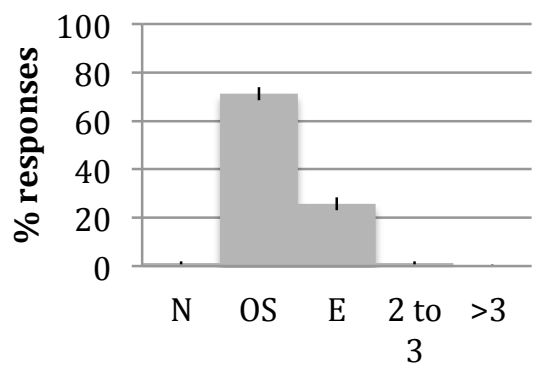

2. Discussion of social issues

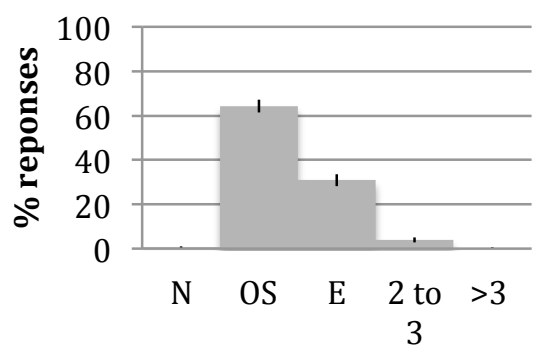

3. Classroom management

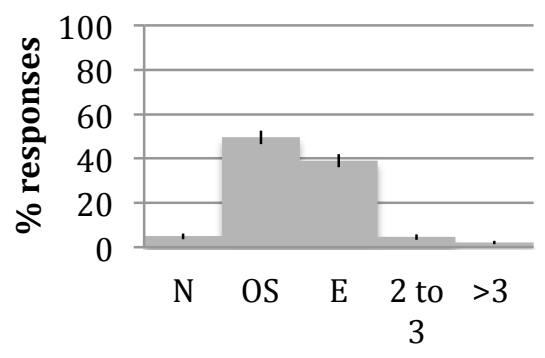

4. Music

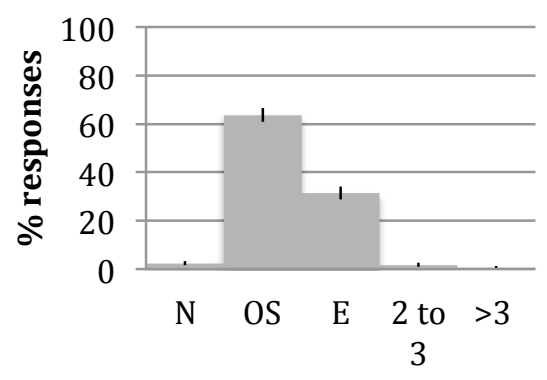

5. Discussion of rules

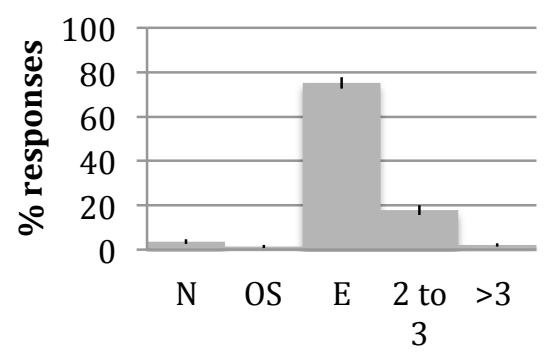

Key:

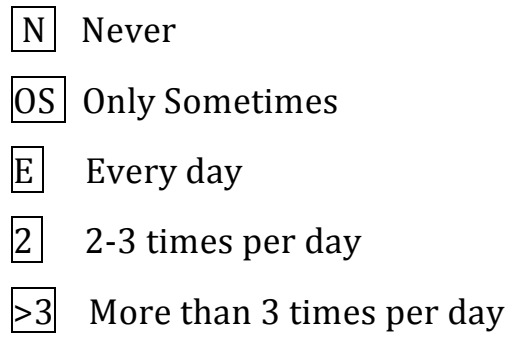

Figure 4.1. Respondents' use of activities presented as percentages with error margins, and with activities shown in the order to which they comprise the first principle component for mat time activities: the social climate.

Figure 4.1 depicts the percentage of responses for the first principle component, relating to the social climate. Discussion of rules was the most frequently occurring activity, with the majority of respondents using it every day or more often. In contrast, games were facilitated the least, with over $70 \%$ respondents using them only sometimes or never. 
1. Explaining a learning activity

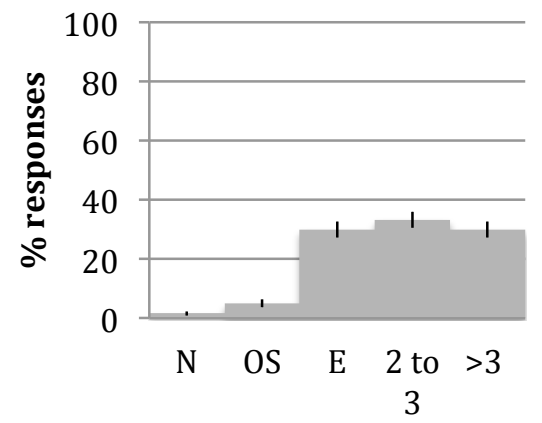

2. Teaching curriculum knowledge

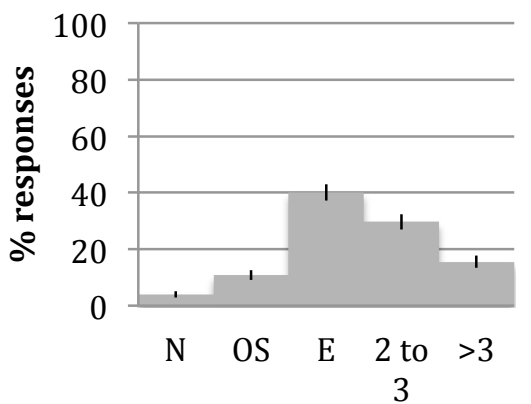

3. Reading a book

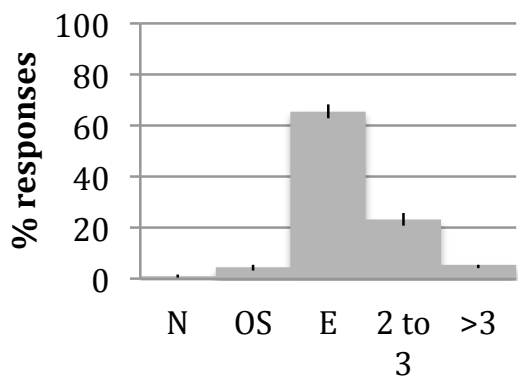

4. Managing transitions

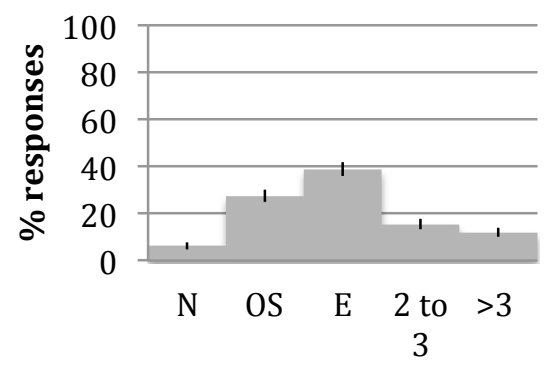

5. Acknowledging good work

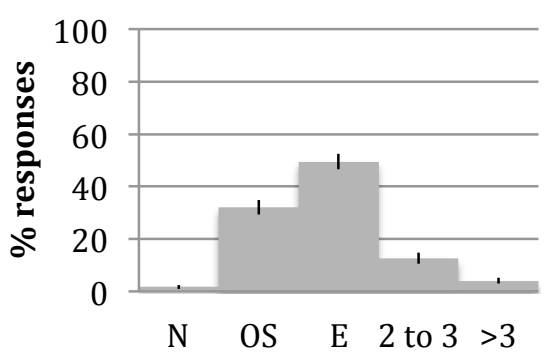

Key:

$\mathrm{N}$ Never

OS Only sometimes

E Every day

2 2-3 times per day

$>3$ More than 3 times per day

Figure 4.2. Respondents' use of activities presented as percentages with error margins, and with activities shown in the order to which they comprise the second principle component for mat time activities: teaching the curriculum.

The activities that were associated with the second principle component, teaching the curriculum are presented in Figure 4.2. These activities each occurred with high frequency, with teaching curriculum knowledge, reading a book, and explaining a learning activity being the most common uses of mat time. 
1. Welcome

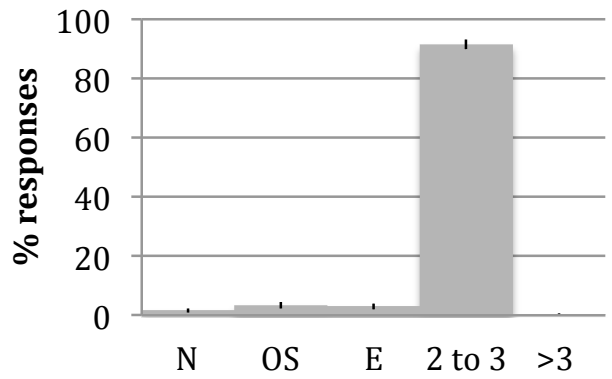

2. News

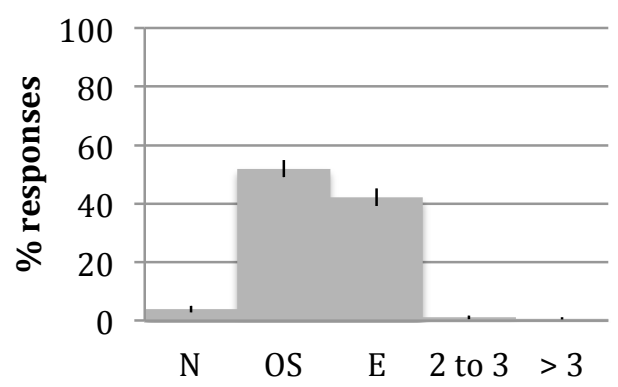

3. Farewell

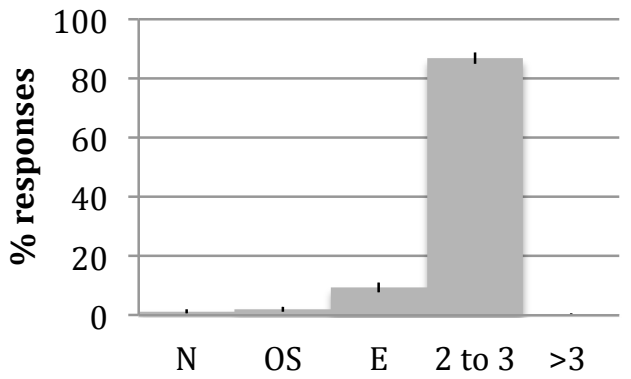

Key:

$\mathrm{N}$ Never

OS Only sometimes

E Every day

2 2-3 times per day

$>3$ More than 3 times per day

Figure 4.3. Respondents' use of activities presented as percentages with error margins, and with activities shown in the order to which they comprise the third principal component for mat time activities: social niceties.

The third principal component pertaining to activities comprised of three ritualised activities that could be used to promote social niceties. These are depicted in Figure 4.3, and show that the respondents predominantly reported that they used the mat for welcome and farewell several times a day. By comparison, 'news' was used significantly less often. 


\subsubsection{Pedagogical goals of mat time}

Teachers were asked to indicate the extent to which they agreed that mat time achieved specific goals on a 5-point Likert scale ranging from strongly disagree to strongly agree. A single factor emerged for the outcomes that teachers expect from mat time and is depicted on Table 4.4. This component had an eigenvalue of 5.00. Where teachers believed that mat time had potential to achieve one of the given goals, there was a tendency for them to nominate other outcomes also.

Table 4.4. The Single Principal Component Principal, Depicting Loadings >.3, for the Pedagogical Goals Item Comprising Respondents' Agreement that Mat Time Contributes to the Achievement of Specific Pedagogical Goals

\begin{tabular}{lr}
\hline Pedagogical goal & Component 1 \\
\% of variance & 55 \\
Language & 0.854 \\
Listening skills & 0.803 \\
Social understandings & 0.777 \\
Establishing daily rhythm & 0.764 \\
Group cohesion & 0.744 \\
Cognitive knowledge & 0.743 \\
Self-control & 0.723 \\
Self-esteem & 0.712 \\
Motor skills & 0.551 \\
\hline
\end{tabular}

All, except motor skills, attracted very high agreement. This was perhaps unsurprising given that activities associated with movement on the mat (activity and games) were nominated less frequently than those that entailed discussion. Furthermore, those goals associated with discussion, such as language skills or listening skills, scored high levels of agreement. The development of group cohesion was nominated with a similarly high frequency (86\%), as well as the development of social understandings (83\%). The development of self-esteem was selected with moderate frequency (65\%). These results are depicted in Figure 4.4 . 
1. Language skills

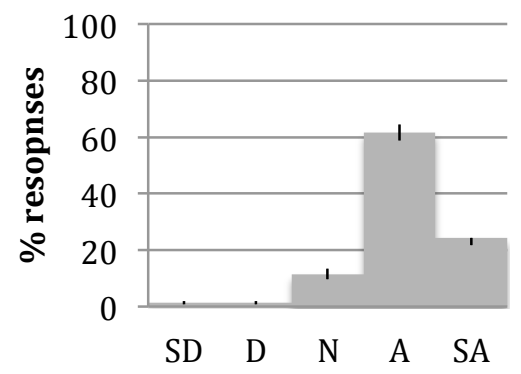

2. Listening skills

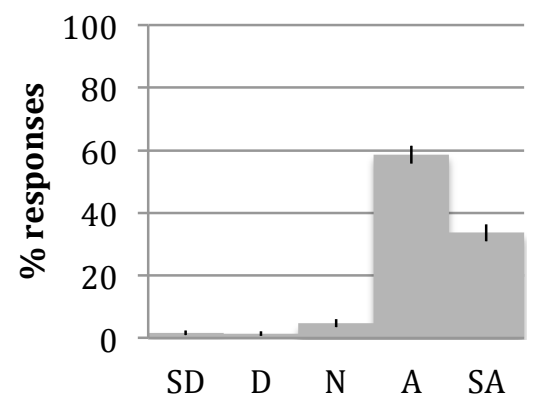

3. Social understandings

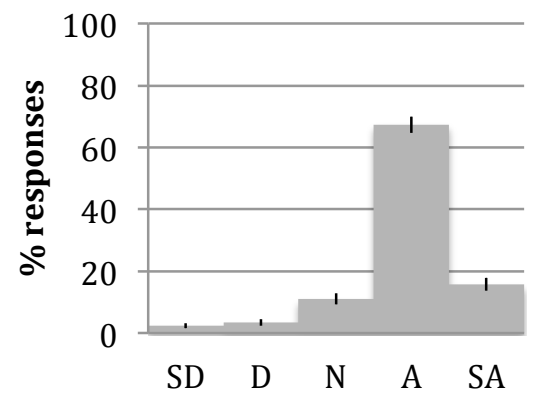

4. Establishing daily rhythm

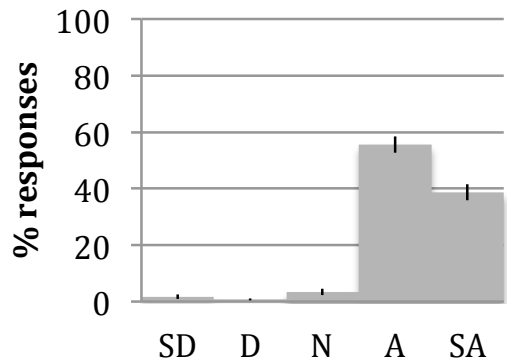

5. Group cohesion

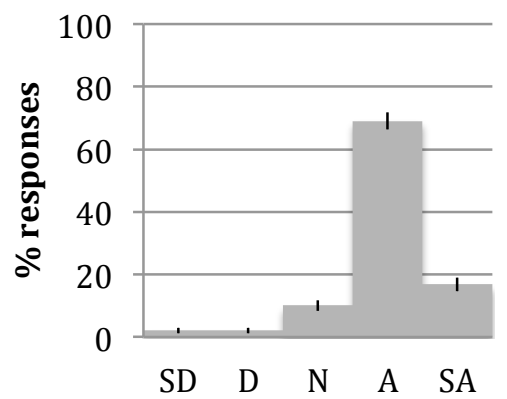

6. Cognitive knowledge

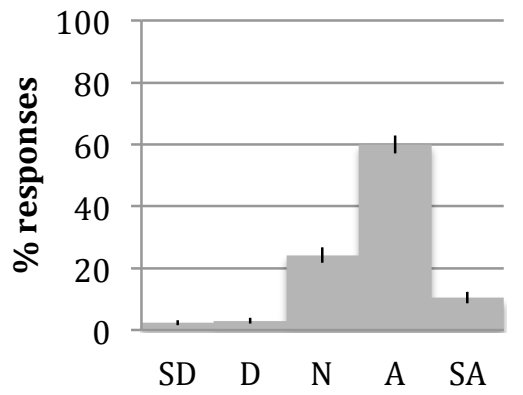

7. Self-control

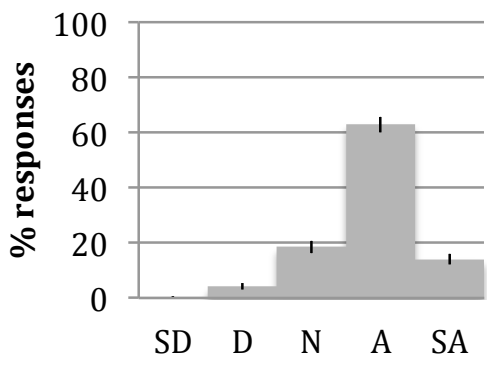

8. Self-esteem

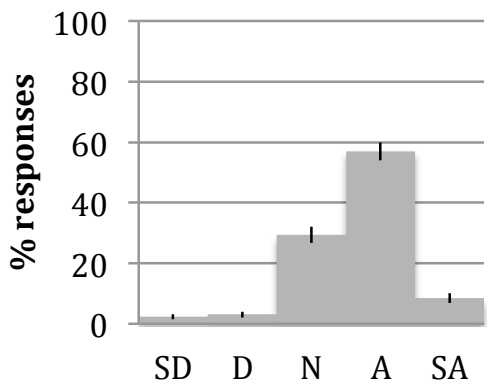

9. Motor skills

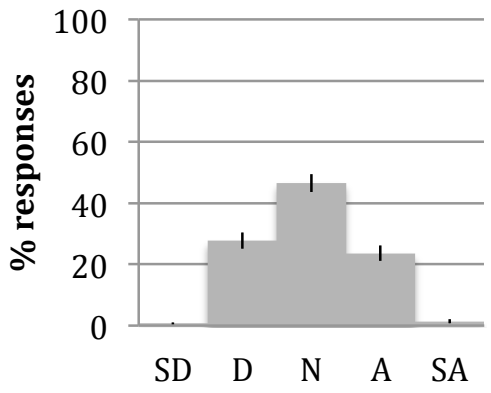

Key

Figure 4.4. Respondents' agreement that mat time achieves pedagogical goals, presented as percentages weith error margins, and with pedagogical goals shown in the order to which they comprise the single principal component.

SD Strongly disagree

D Disagree

$\mathrm{N}$ Neutral

A Agree

SA Strongly agree 


\subsubsection{Children's behaviours and feelings}

Teachers were asked to indicate their agreement with a range of statements about children's behaviour at mat time, using 5-point Likert scales that ranged from strongly disagree to strongly agree. Two components emerged, summarising children's behaviours, and are shown on Table 4.5. The first had an eigenvalue of 3.60 and included the following items about certain children: some consistently dominate, some consistently observe, some consistently put their hands up, and some consistently behave in disruptive ways. These are observable differences about specific children's participation and imply that there are discrepancies in active participation at mat time across the group of children.

The second component concerning children's behaviour had an eigenvalue of 1.06 , which indicated that this component was very marginal. It related to teachers' beliefs regarding children's attentiveness and enjoyment. In other words, this component described children's positive feelings at mat time.

Table 4.5. The Two Principal Components Depicting Loadings >.3, for the Item on Children's Behaviours and Feelings at Mat Time Comprising Teachers' Agreement to Observable Behaviours and Positive Feelings

\begin{tabular}{lrr}
\hline Behaviour & \multicolumn{2}{r}{ Component } \\
& \multicolumn{2}{c}{1} \\
\hline \% of variance & & 14 \\
& 0.896 & \\
Some children dominate & $\mathbf{0 . 8 9 2}$ & \\
Some children observe only & $\mathbf{0 . 8 4 3}$ & \\
Some children always put their hands up & $\mathbf{0 . 7 4}$ & \\
Some children are consistently disruptive & & $\mathbf{0 . 9 4}$ \\
All children are attentive & 0.516 & $\mathbf{0 . 6 9 3}$ \\
Children enjoy mat time & & \\
\hline
\end{tabular}


The frequency of responses pertaining the principle component, associated with observable behaviours, is depicted as Figure 4.5, and shows that the trend was for high frequencies of teachers to agree or highly agree with the presence of each behaviour. For example, the highest level of agreement was that certain children consistently put their hands up (83\%) and the lowest level of agreement was that certain children consistently disrupted mat time (48\%).

\section{Some children dominate}

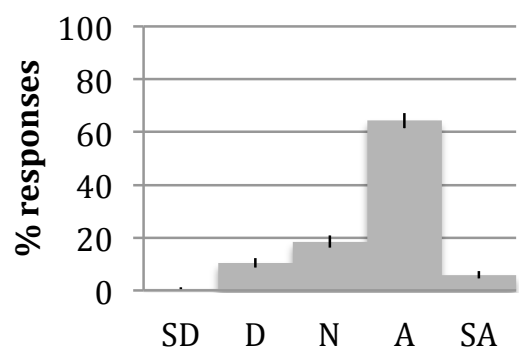

2. Some children observe only

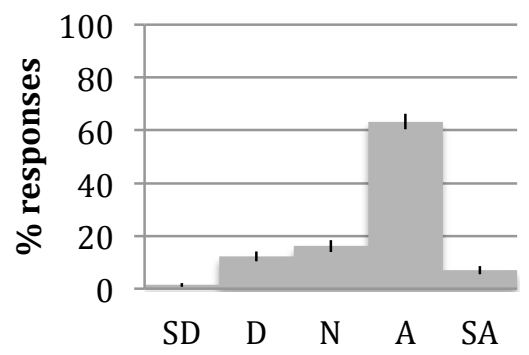

Key

SD Strongly disagree

D Disagree
3. Some children always put their hands up

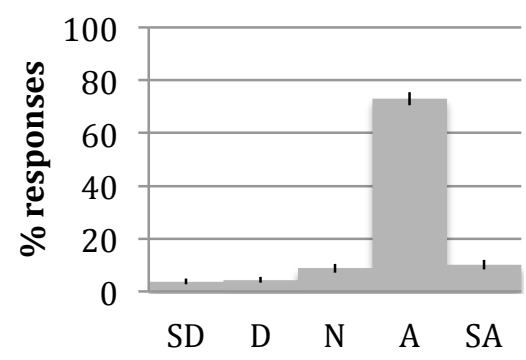

4. Some children are always disruptive

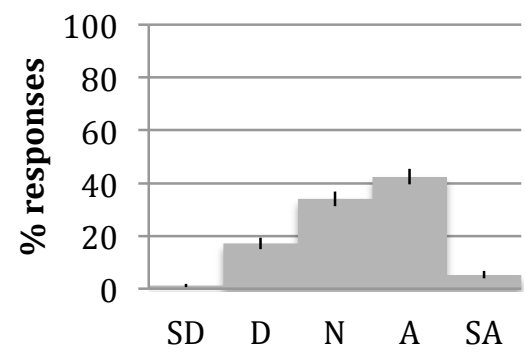

N Neutral

A Agree

SA Strongly agree

Figure 4.5. Respondents' agreement on children's behaviours and feelings at mat time depicted as percentages with error margins, and with respondents' agreement on shown in the order to which they comprise the first principal component: observable behaviours. 
Figure 4.6 provides a summary of teachers' ratings relating to the principal component associated with children's positive feelings about mat time. A high percentage of teachers agreed or strongly agreed with the statement that children enjoy mat time (79\%). Nevertheless, responses to the statement that children are attentive at mat time indicated significantly less agreement (18\%).

1. All children are attentive

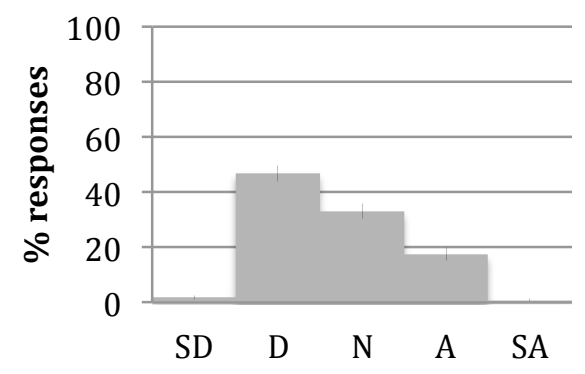

Key

SD Strongly disagree

D Disagree
2. Children enjoy mat time

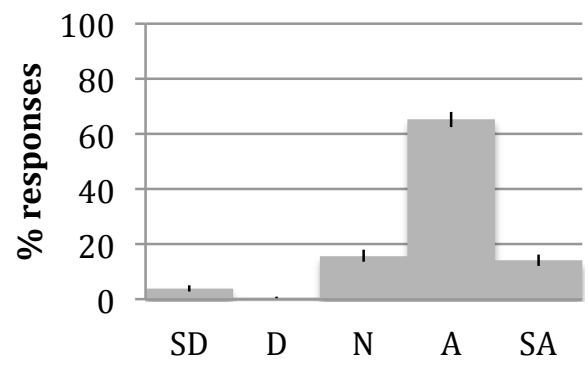

N Neutral

A Agree

SA Strongly agree

Figure 4.6. Respondents' agreement on children's behaviours and feelings at mat time depicted as percentages with error margins, and with respondents' agreement on shown in the order to which they comprise the second principal component: positive feelings.

\subsubsection{Strategies for engaging children}

This question asked teachers to select strategies that they use to maintain children's engagement from a list. This created two categories for each strategy in terms of the teachers who used the particular strategy, and those who did not. Two components were identified, and are summarised in Table 4.6. The first component had an eigenvalue of 3.20 and included: issuing reprimands, writing children's name on the board (encouragement for compliant behaviour and as a deterrent for non-compliant behaviour), removing children from the mat, giving 
rewards, and seating specific children close to the teacher. These can be described as strategies that use behavioural reinforcement.

Table 4.6. The Two Principal Components Depicting Loadings >.3, For the Item on Teachers' Reports on Their Use of Behaviourist Strategies and Appealing to Children's Attentiveness in Order to Engage them at Mat Time

\begin{tabular}{lrr}
\hline Strategies & & Component \\
& 1 & 14 \\
\hline \% of variance & 46 & \\
& & \\
Issuing reprimands & $\mathbf{0 . 7 9 5}$ & \\
Writing a child's name on the board & $\mathbf{0 . 7 7 4}$ & $\mathbf{0 . 7 2 5}$ \\
Removing children from the mat & $\mathbf{0 . 7 1 3}$ & $\mathbf{0 . 8 9 4}$ \\
Giving rewards & $\mathbf{0 . 6 8 8}$ & $\mathbf{0 . 6 5 8}$ \\
Placing a child in proximity to teacher & & \\
Using interesting voice intonation & 0.349 & \\
Incorporating children's interests & & \\
\hline
\end{tabular}

The second component was marginal; it had an eigenvalue of 1.00 and included: using voice intonation and incorporating children's interests. These specific teaching strategies are more likely to appeal to the children's enthusiasm than those outlined in the previous section (Emilson, 2007). Specifically, they are strategies that potentially catch children's attention and hold their engagement in the mat time content or activity (see Mitchell, 1993). Eggen and Kauchak (2006) suggest that when teachers use such strategies, they potentially model enthusiasm for learning; the children observe the teacher's enthusiastic approach and might imitate through their own attentiveness to the learning.

Figure 4.7 shows the percentages associated with the various teaching strategies. The most common strategy nominated was to move certain children closer to the teacher (53\%). On the other hand, removing children from the mat area was nominated the least (21\%). Given the prevalence in the literature of examples 
where teachers issued reprimands (see Emilson, 2007; Rubenstein Reich, 1994) fewer teachers than expected indicated that it was a strategy that they used (34\%).

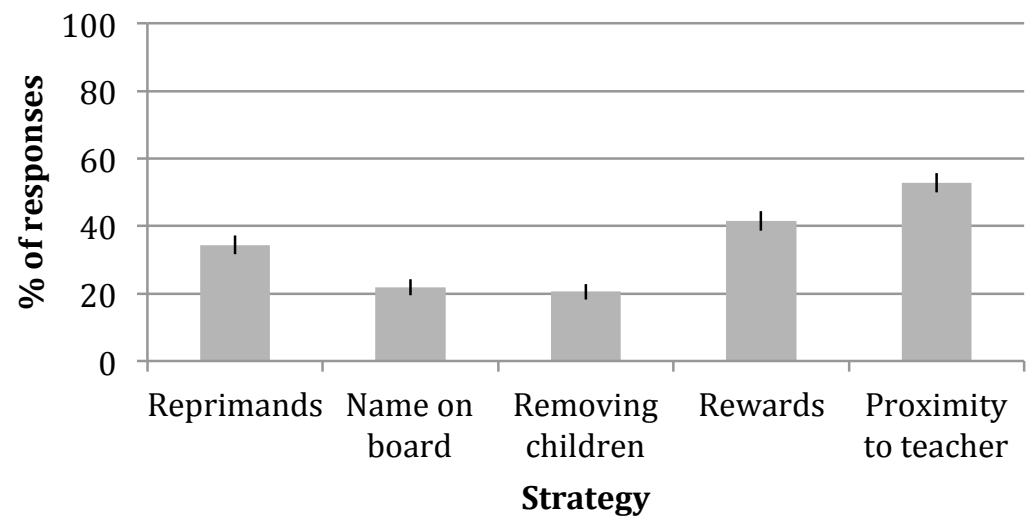

Figure 4.7. The respondents' use of strategies to engage children at mat time depicted as percentages of responses with error margins, and shown in the order to which they comprise the first principal component: behavioural reinforcement.

Figure 4.8 shows that teachers' use of strategies to engage children's attention, and that appealed to their interests, typically comprised using intriguing voice intonation; however, other ways of appealing to children's interests was nominated by $56 \%$ of the respondents. This was far lower than expected.

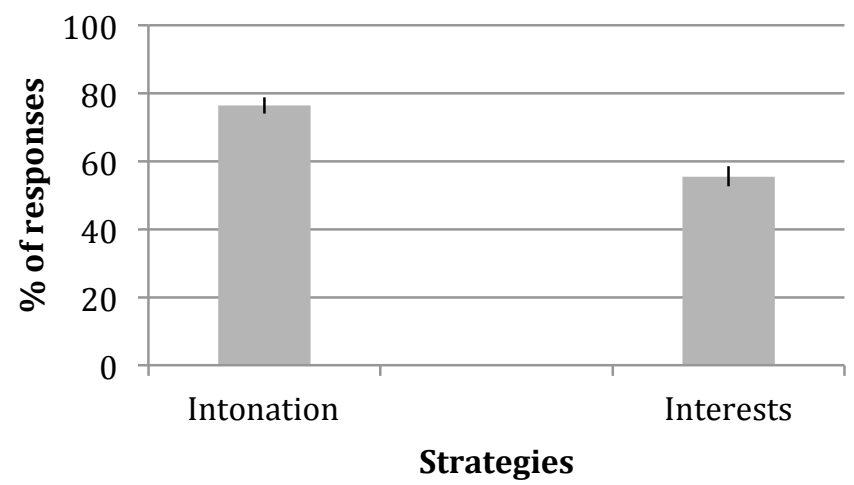

Figure 4.8. The respondents' use of strategies to engage children at mat time depicted as percentages of responses with error margins, and shown in the order to which they comprise the second principal component: appealing to children's interests. 


\subsubsection{Degree of challenge experienced by pedagogical tensions}

Respondents were presented with a range of conflicting pedagogical goals and were asked to indicate how challenging he or she found each one to resolve. Scales were provided that ranged from extremely challenging to extremely easy. One factor emerged about challenges that teachers faced when facilitating mat time and this is depicted in Table 4.7. There was an eigenvalue of 3.49. The results indicated that if teachers found one aspect of mat time challenging, they were likely to find all of the given aspects challenging.

Table 4.7. The Single Principle Component, Depicting Loadings >.3, for the Item on Teachers' Reports of Degree of Challenged Experienced in Resolving Pedagogical Tensions at Mat Time

\begin{tabular}{lr}
\hline Potential challenge & Component 1 \\
\hline \% of variance & 70 \\
Maintaining all children's engagement & 0.859 \\
Ensuring the participation of all children & 0.858 \\
Balancing individual and group needs & 0.832 \\
Managing non-compliance & 0.825 \\
Balancing the curriculum with children's interests & 0.806 \\
\hline
\end{tabular}

The highest frequency of teachers rated each of the various items as sometimes challenging and sometimes easy, which is depicted on Figure 4.9. The range was from $34 \%$ to $58 \%$ for ensuring that all children participate and balancing the needs of the individual with the needs of the group respectively. Given that Rubenstein Reich (1994) described many of these challenges as the cause of significant difficulty for teachers, it was surprising to see few teachers in the present study nominate any difficulty. 
1. Maintaining all children's engagement

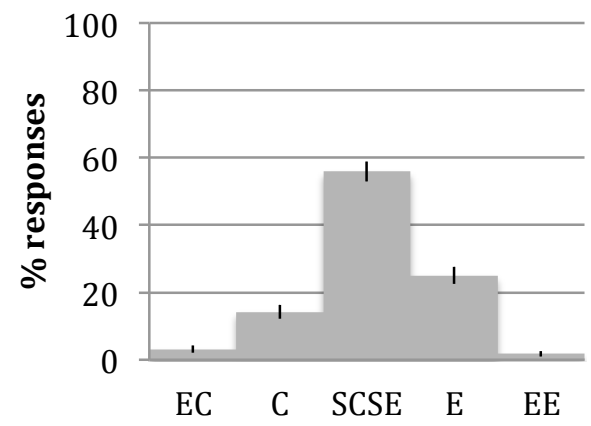

2. Ensuring all children's participation

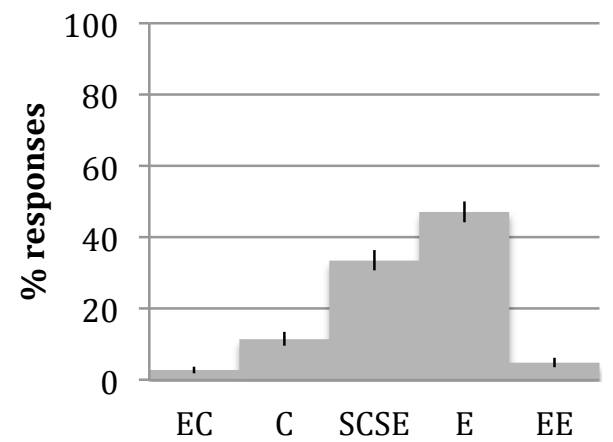

3. Balancing individual and group needs

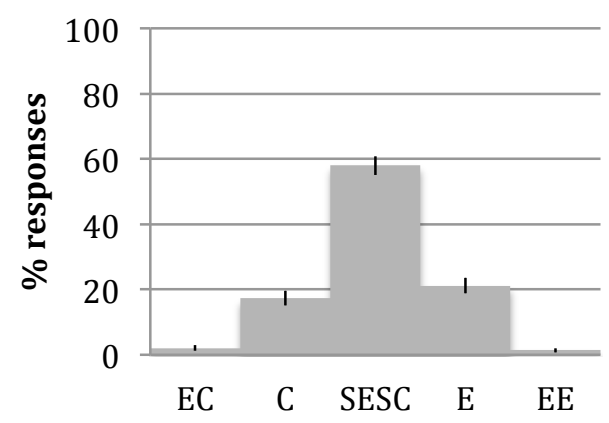

4. Managing non-compliance

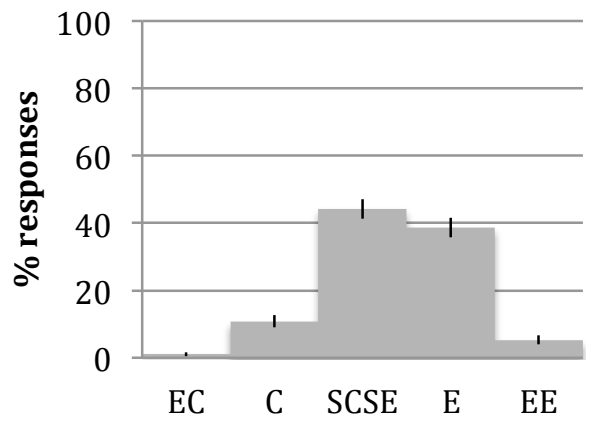

5. Curriculum vs children's interests

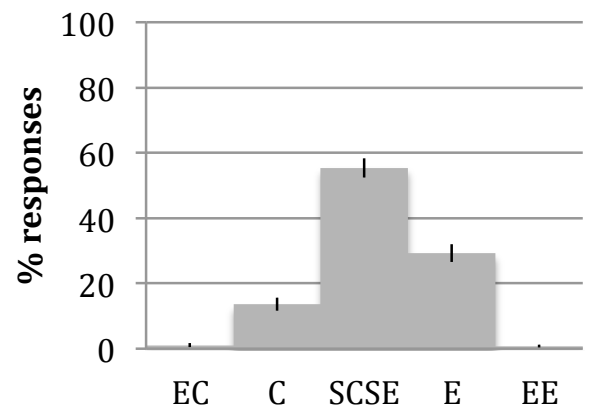

Key

EC Extremely challenging

C Challenging

SCSE Sometimes easy, sometimes challenging

E Easy

EE Extremely easy

Figure 4.9. The respondents' ratings of the degree of challenge associated with pedagogical tensions, depicted as percentages of responses with error margins, and shown in the order to which they comprise the single principle component. 


\subsubsection{Pedagogical approaches at mat time}

Teachers were asked to place their pedagogies on continua representing two opposing constructs; for example, one continuum comprised informal pedagogy at one pole and formal at the other. One dominant component emerged and is represented in Table 4.8 with an eigenvalue of 2.87. This means that teachers who reported that their practices were discursive, also tended to report that they issued a high number of questions to children, and viewed their mat times as planed, formal, and child-directed.

Table 4.8. The Single Principal Component, Depicting Loadings >.3, for the Respondents' Reports on the Pedagogical Approaches Used at Mat Time

\begin{tabular}{lr}
\hline Approach & Component 1 \\
\hline \% of variance & 57.3 \\
& \\
Instructive to 'discussive' & 0.808 \\
Low frequency of questions to high & 0.8 \\
Unplanned to planned & 0.773 \\
Informal to formal & 0.722 \\
Teacher-directed to child-directed & 0.673 \\
\hline
\end{tabular}

Overall, responses were fairly balanced between the extremes of the continua. Responses were weighted toward a high frequency of question-asking by the teacher with $62 \%$ of the responses in the highest categories. This is of interest, because several studies suggest that teachers incorporate a specific questioning style, known as the IRF sequence that is not conducive to class discussion (Johnston, 2004; Raban, 2001; Wells, 1993). Only 38\% nominated their practices as predominantly discursive. The teachers' responses also showed that when they did not choose the midpoint, they tended to rate their teaching approach as planned (46\%) as opposed to unplanned (13\%), and teacher-directed (44\%) rather than child-directed (11\%). The degree of formality either side of the midpoint was comparable, with $22 \%$ rating their practices as formal and $29 \%$ rating them as informal. These percentages are depicted in Figure 4.10. 
1. Instructiveness to 'discussive'

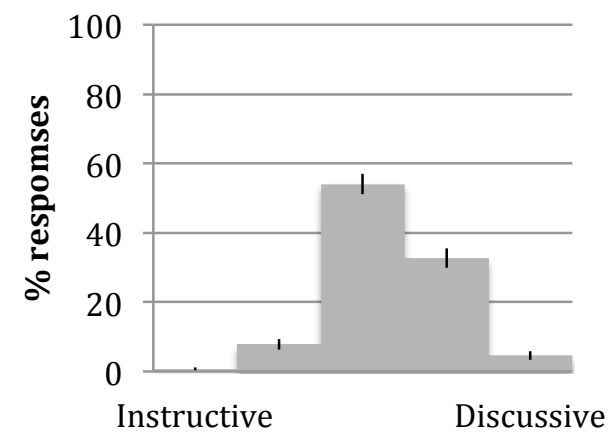

2. Low frequency of questions to high

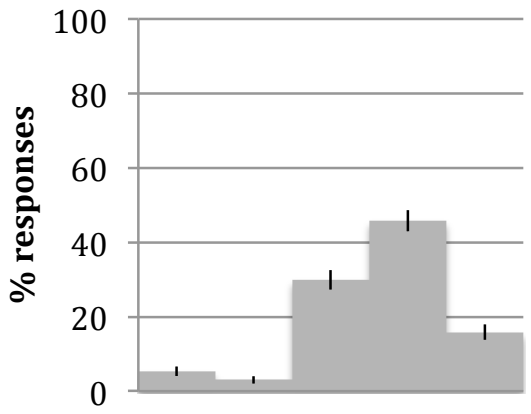

Low frequency High frequency
4. Informal to formal

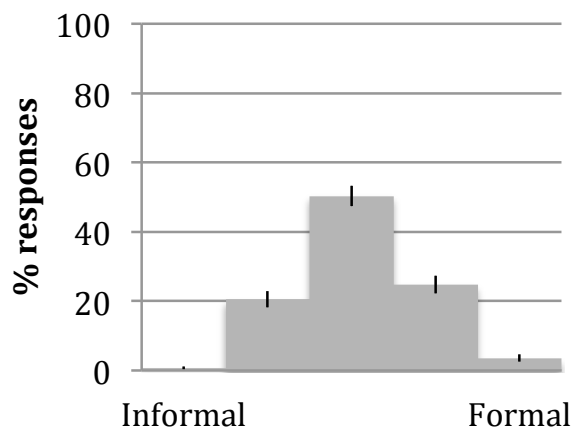

5. Teacher-directed to child-directed

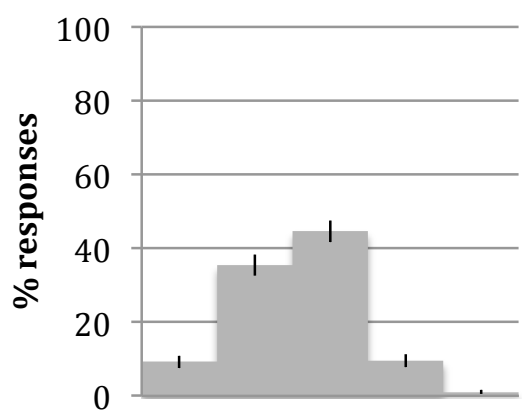

Teacher directed Child directed

3. Unplanned to planned

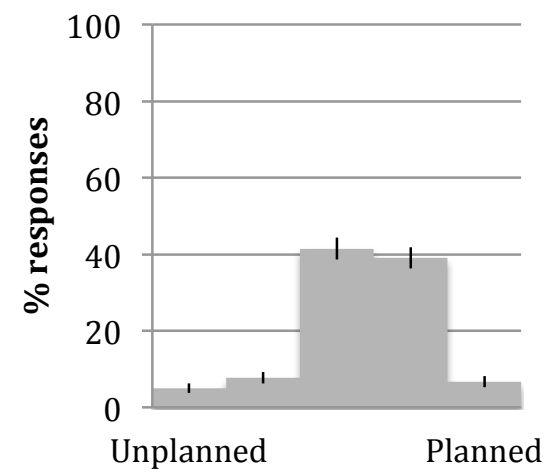

Figure 4.10. Respondents' rating of their pedagogical approaches at mat time, presented as percentages with error margins, and shown in the order to which they comprise the single principal component: pedagogical approaches. 


\subsubsection{Likelihood of all children participating}

In a stand-alone item, the respondents were asked to rate the likelihood that every child participated or contributed at mat time. The distribution, presented in Figure 4.11, indicated that many respondents believed that it was likely or very likely that all children participated (58\%); however, a significant proportion indicated the converse; that it was unlikely or very unlikely (33\%). Only $9 \%$ were undecided.

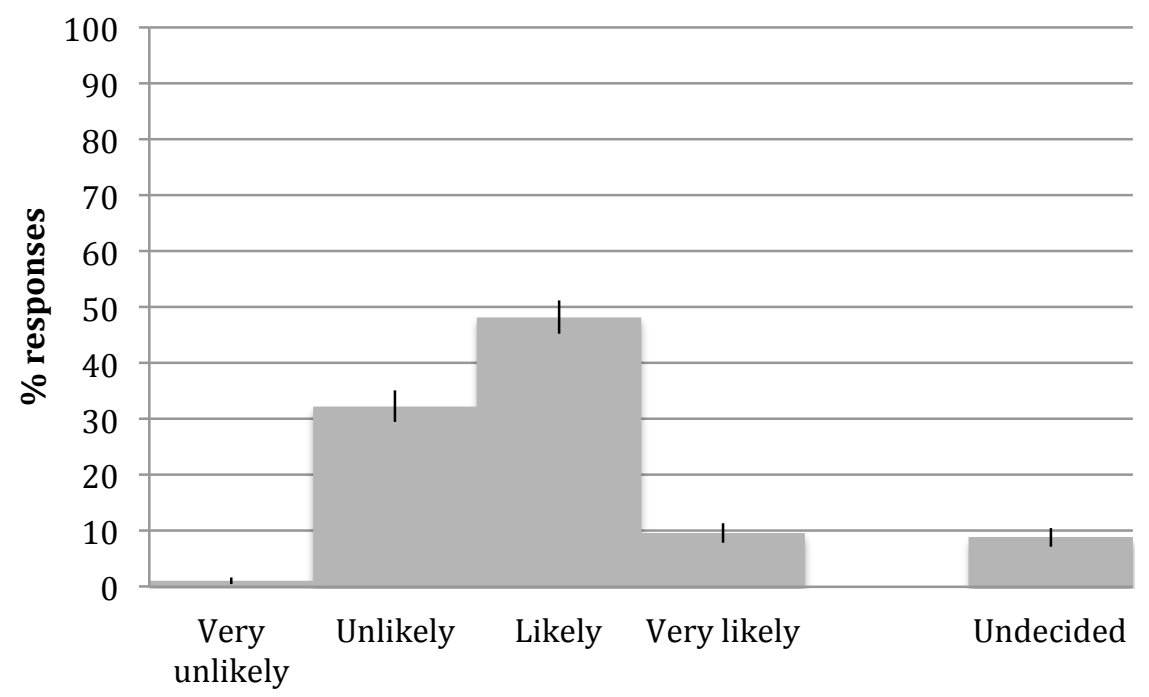

Figure 4.11. Respondents ratings on the likelihood that all children participate at mat time, depicted as percentages with error margins.

\subsubsection{Teachers' reports on incorporating children's interests and comparison of their ratings of children's behaviour}

Thus far, the survey results have indicated that fewer than $20 \%$ of the respondents perceive children to be attentive at mat time. More over, a third of participating teachers reported that not all children participate during activities on the mat and, in fact, $70 \%$ agreed that certain children merely observe, rather than directly participating. In addition, nearly half of the respondents agreed that some children consistently behave in disruptive ways. When viewed together, these results indicate that some children are withdrawn, disengaged, or inattentive at mat time. 
Scholars who have researched students' attentiveness in general classroom situations, contend that teachers can enhance students' engagement by drawing on those things that interest them (Ainley, Hidi, \& Berndorff, 2002; Hidi \& Renninger, 2006; Schraw, Flowerday, \& Lehman, 2001). This is important, because engagement in classroom tasks has been associated with students' feelings of selfefficacy (Marsh, Köller, Trautwein, Lüdtke, \& Baumert, 2005), motivation and academic performance (Hidi \& Harackwitz, 2000).

Several authors have suggested that children's on-task behaviour and active participation on the mat might be enhanced when teachers facilitate activities and topics that are interesting and relevant to children (Canney \& Byrne, 2006; Emilson, 2007; Gallas, 1992; Murphy, 2003; Rubenstein Reich, 1994; Zaghlawan \& Ostrosky, 2011). However, few of these authors were able to investigate whether teaching strategies that appeal to children's interests do, indeed, engage their attentiveness and participation at mat time. Therefore, the present study made comparisons between the reports of teachers who indicated that they considered children's interests at mat time, and those who did not.

Paired samples $t$-tests were conducted to compare teachers' responses about their use of children's interests with their ratings of children's observable behaviours, and positive feelings relating to engagement at mat time. The behaviours that teachers' observed were: specific children merely observing, behaving disruptively, putting their hand up, and dominating. The positive feelings included teachers' reports that children are attentive and enjoy mat time. The data used for the $t$-tests were the calibrated measurement scale data. These are represented in Figure 4.12. 


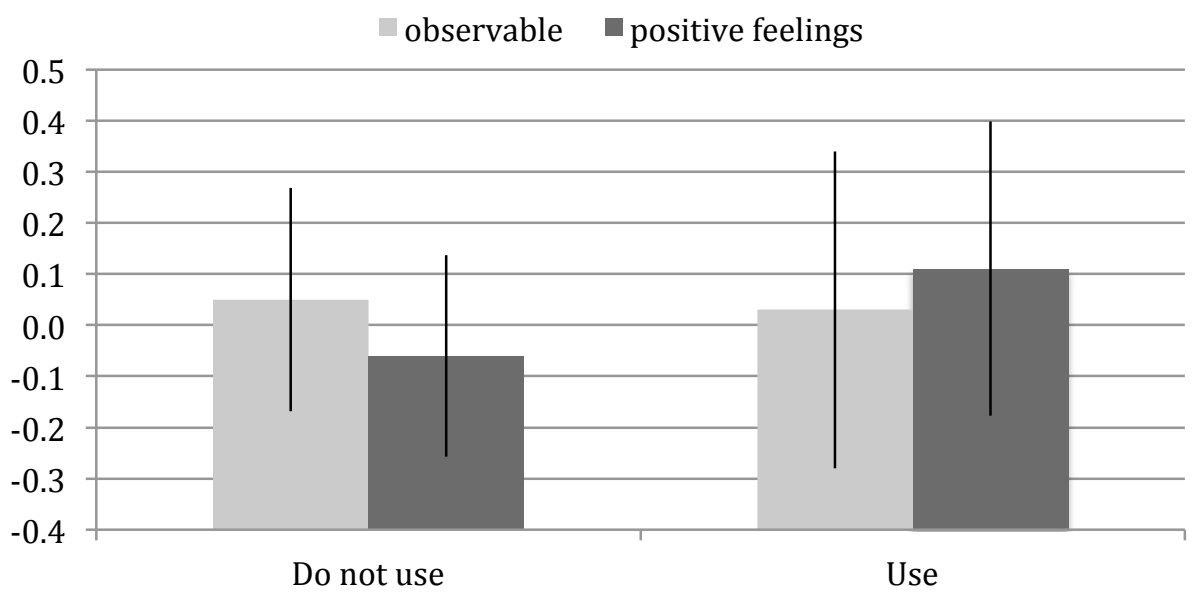

Figure 4.12. Results of the paired sample $t$-tests: Comparison of teachers' reports of children's observable behaviours (observing, disrupting, dominating, putting hands up) and positive feelings (enjoyment and attentiveness), depicting the differences between those who report appealing to children's interests and those who do not.

Comparisons of children's observable behaviours indicated no significance between teachers who use interests $(M=-.03, \mathrm{SD}=2.5, \mathrm{SEM}=.19)$, and teachers who do not $(M=.05, \mathrm{ST}=2.4 ; \mathrm{SEM}=.22) ; \mathrm{t}<1$. These results suggest that both groups of teachers have similar ratings of observable behaviours of specific children either consistently dominating or merely observing as well as certain children participating by consistently putting their hands up or disrupting the mat time. In other words, the use of children's interests makes no difference to teachers' rating of observable behaviours.

It was predicted that teachers who use children's interests might report greater enjoyment or attentiveness (positive feelings) compared with those who do not. In fact there was no statistical difference between the group that does use interests $(M=.11, \mathrm{SD}=3.7, \mathrm{SEM}=.29)$ and the group who does not $(M=-$ $.06, \mathrm{SD}=3.4, \mathrm{SEM}=.31$ ); $\mathrm{t}<1$. An inference suggested by this result is that teachers' ratings of children's enjoyment and attentiveness are not influenced by whether or not they use children's interests. 


\subsubsection{Teachers' reports on incorporating children's interests and comparison of the degree of challenge presented by pedagogical tensions}

Alexander, Johnson, Leibham, and Kelley (2008) canvassed the interests of 215 North American 4 year olds. They posited that although children demonstrate a wide-array of interests before formal schooling, teachers might find it difficult to cater for these interests at school; curricular objectives impede teachers' abilities to be responsive to children's individual interests. New Zealand teachers have similar demands made of them with regard to administering the curriculum while embracing constructivist pedagogies. The present study investigated whether New Zealand teachers who reported that they appealed to children's interests at mat time experienced additional pedagogical tensions compared with those teachers who did not. The pedagogical tensions in question were: maintaining all children's engagement, ensuring the participation of all children, balancing the needs of the individual with the needs of the group, managing non-compliance, and balancing curriculum delivery with children's interests

Respondents were asked to indicate their level of agreement about the degree of challenge experienced with a range of pedagogical tensions. Paired sample $t$-tests indicated that there was no statistically significant difference between those who use interests $(M=.22, \mathrm{SD}=2.6, \mathrm{SEM}=.20)$ and those who do not $(M=-$ $.29, \mathrm{SD}=2.9, \mathrm{SEM}=.23) ; t(286)=1.6, \mathrm{p}=0.9$. In other words, as shown by Figure 4.13 , the reported use of interests was unrelated to the extent to which teachers found any of the pedagogical tensions challenging to resolve. 


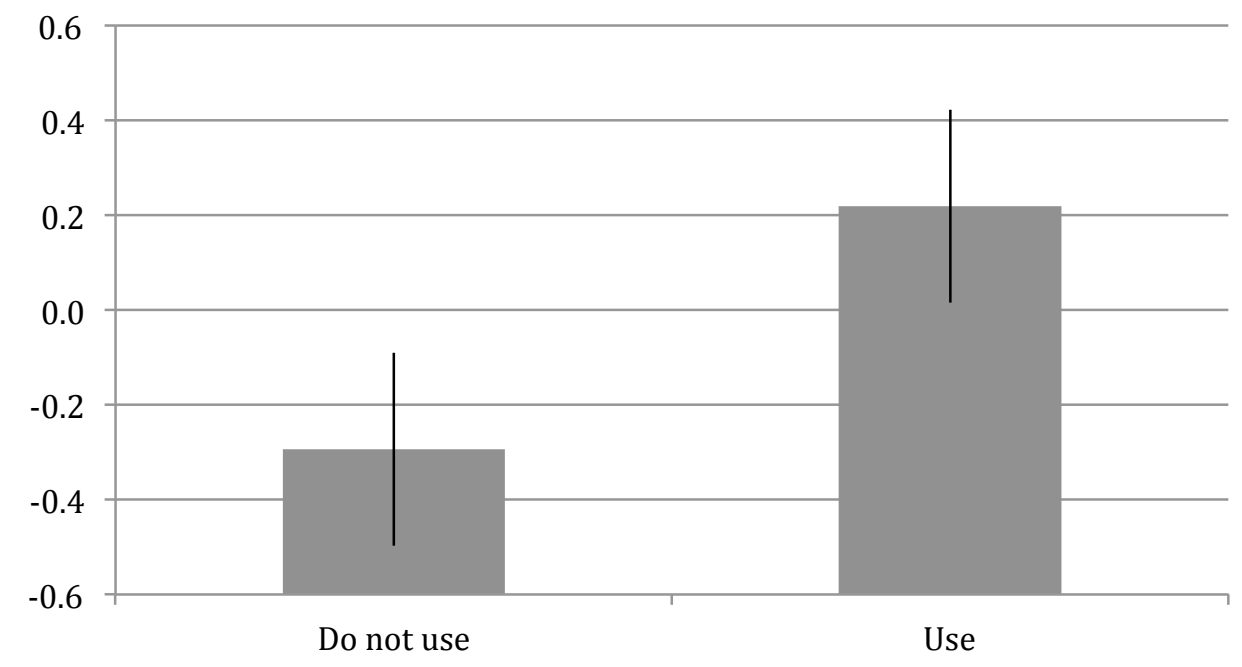

Figure 4.13. Results of paired sample $t$-tests: Comparison of teachers' reports on whether pedagogical tensions at mat time are challenging to resolve, depicting the differences between those who report appealing to children's interests and those who do not.

\subsubsection{Comparison of teachers' reports on appealing to children's interests and their pedagogical approaches}

Several studies have suggested that teachers' pedagogical approaches might be more amenable to drawing on children's interests than others. For instance, Joussemet, Koestner, Lekes, and Houlfort (2004) investigated conditions that enhanced 106 children's engagement in tasks in grades 1-5 at a single school in Montreal. The findings indicated that pedagogy that enabled children's autonomy, encouraged their initiatives, and minimised the controlling language of teachers, were more likely to result in children's reported enjoyment and engagement than those that did not. Similarly, Wigfield and Cambria's (2010) review of the interest literature asserted that teachers must ensure that tasks have relevance to students' interests and lives in order to engage them. Establishing relevance might involve pedagogies that call on children's ideas and input, or that draw on childdirected strategies (Schraw et al., 2001). 
Chi-square tests were undertaken to investigate whether teachers who reported drawing on children's interests also used reported using pedagogies that allowed children input and autonomy (for example, child-directedness, or spontaneous as opposed to planned). Figure 4.14 depicts the comparisons of teachers' reports of their pedagogical approach and engaging children's attention by incorporating their interests.

1. Teacher-directedness

No use of interests $\square$ Use of interests

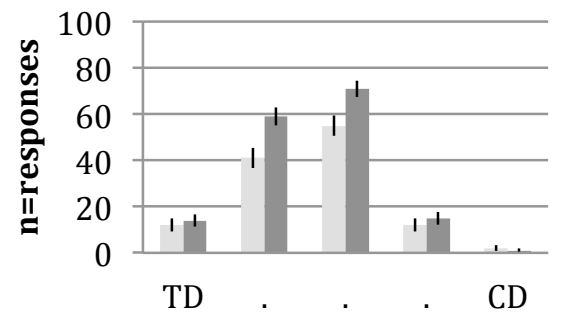

2. Frequency of questions

No use of interests $\square$ Use of interests

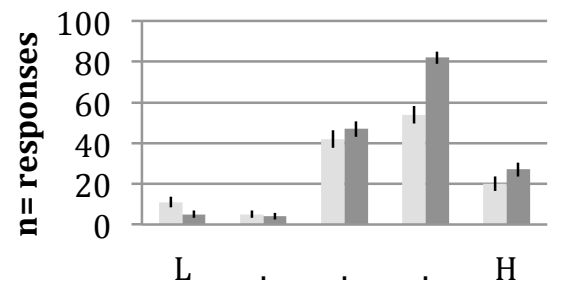

3. Extent of planning

No use of interests $\square$ Use interests

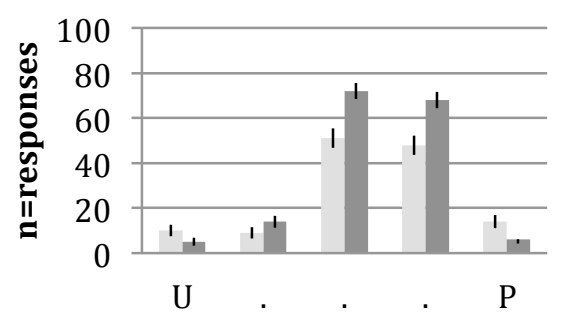

4. Formality

No use of interests Use interests

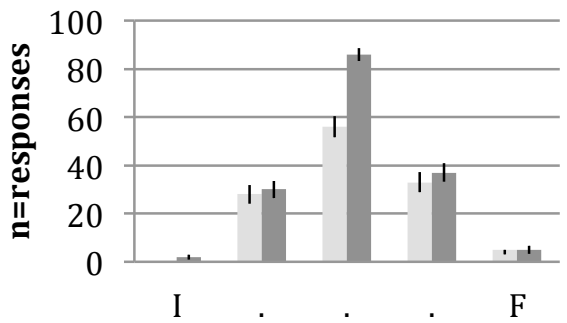

5. Level of instructiveness

No use of interests Use interests

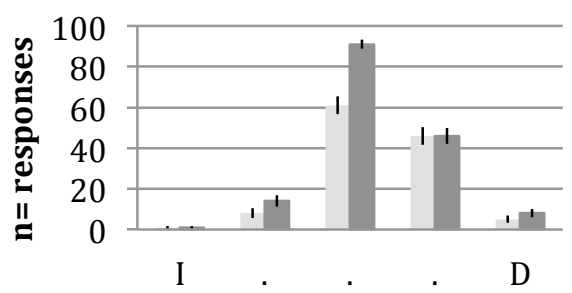

Key

(TD) teacher-directed to (CD) child directed

(L) low frequency of questions to $(\mathrm{H})$ high frequency

(U) unplanned to $(\mathrm{P})$ planned

(I) informal to (F) formal

(I) instructive to (D) 'discussive'

Figure 4.14. Comparison of teachers' reports on their pedagogical approaches at mat time and whether they appeal to children's interests or not. 
The chi-square tests showed no significant differences between respondents' reporting use of children's interests and their reporting no use of interests in respect of the various teaching strategies. There was no significant difference with respect to (1) teacher-directedness; $\chi(6)=4.14, p=0.6,(2)$ frequency of questions directed at the children; $\chi(6)=5.85, \mathrm{p}=0.44$, (3) degree of planning; $\chi(6)=9.44, \mathrm{p}=0.15,(4)$ degree of formality; $\chi(6)=6.72, \mathrm{p}=0.35$, and (5) level of instructiveness or 'discussiveness'; $\chi(6)=6.92, \mathrm{p}=0.33$.

\subsubsection{Comparison of reported use of interests and ratings of the likelihood that all children participate}

In the present study, one third of teachers said that it was unlikely that all children participate at mat time. Children's active participation at mat time has been linked to quality of learning with regard to young children's book comprehension (Mandel Morrow \& Smith, 1990) and enhanced vocabulary (Han et al., 2005). In addition, a student's effective participation might positively impact on his or her self-concept (Bukowski et al., 2011; Hughes \& Zhang, 2007).

Foster et al. (2009) assert that promoting students' participation requires foci that are relevant to those students' interests; however, very little empirical research could be found to corroborate this view, especially with regard to young children in classroom situations. Notwithstanding, Zaghlawan and Ostrosky (2011) found that children seemed to be more on-task when the mat time activity appeared interesting to them.

A chi-square test was used to investigate the relationship between teachers' indication that they use children's interests and the reported likelihood that all children participate or contribute. The $p$-value indicated significance; 
$\chi(6)=22.71, \mathrm{p}<001^{5}$. This result suggested that teachers who reported using strategies that appealed to children's interest also reported that it was likely that all children participated or contributed. These results are shown in Figure 4.15 .

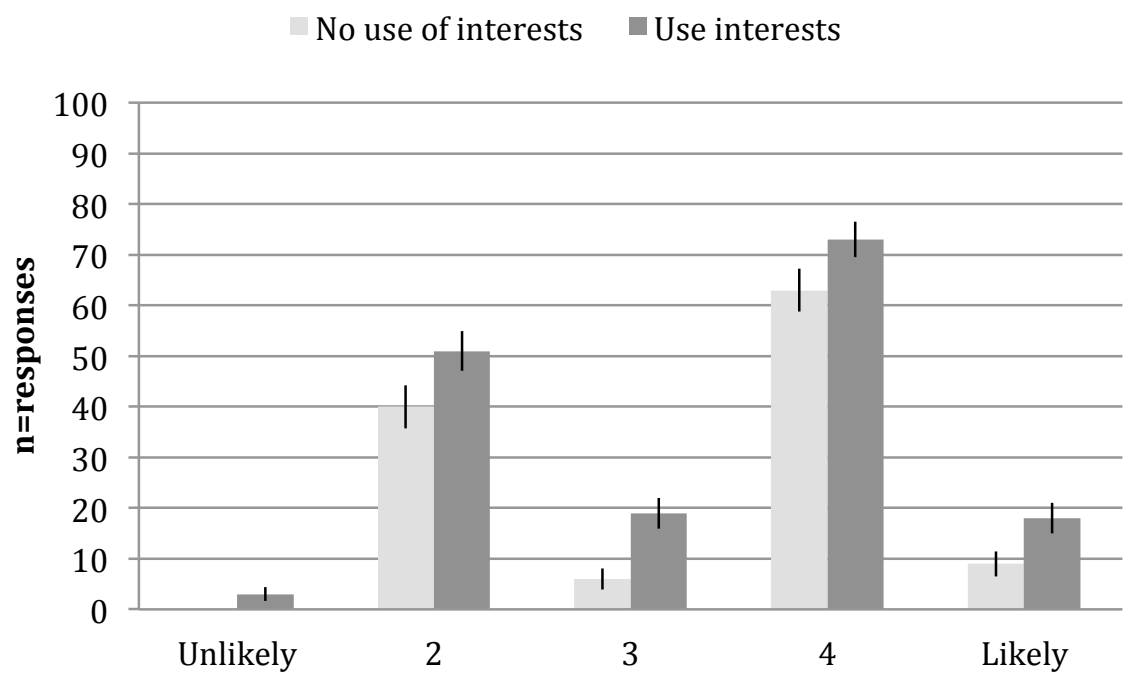

Figure 4.15. Chi test results on teachers' ratings of the likelihood that all children participate, depicted as frequency of responses with error margins, and comparing teachers who report appealing to children's interests and those who do not

\subsubsection{Comparison of teachers' ratings of the likelihood that all children participate with (1) the pedagogical approach used, and (2) children's behaviours at mat time}

A primary focus of the present study is the interactional milieu of mat time. One aspect of this milieu is how teachers and children's behaviours inter-relate. Hong (1995) provides an apt illustration between the inter-relationship between a teacher's pedagogy and her perception of children's mat time behaviour; the teacher used highly teacher-directed strategies, such as issuing commands and choosing the topic in a bid to promote specific mat time behaviours to children, namely sitting 'nicely' and not calling out. The teacher's belief was that these

${ }^{5}$ The actual $p$ value is 0.0009 
specific pedagogical strategies were the most effective achieving those childbehaviours that she desired.

In order to investigate whether there was any bearing on teachers' reports of their pedagogical approach upon their reports of the presence of children's diverse participatory behaviours, a 2 × 2 ANOVA was conducted. The pedagogical approaches that included were child-directed and teacher-directed. These were correlated with teachers' agreement with the presence of specific child behaviours pertaining to participation, or not; namely that certain children consistently dominated, observed, disrupted, put their hands up, or merely observed.

There was a weak correlation between respondents' reports about the likelihood that all children participated and the teaching approach used, $\rho(297)=.21$. The likelihood of all children participating was minimally correlated with teaching pedagogy that was child-directed rather than teacher-directed.

Whereas teacher's behaviours might influence children's participatory behaviours, it seems likely that specific children's behaviour might also influence the participation of their peers; for instance, Danielewicz et al., (1996) noticed that one child found it very difficult to maintain a participatory role when other peers dominated the mat time. A second $2 \times 2$ ANOVA was conducted that comprised teachers' agreement, or not, that children had diverse participation roles and the likelihood, or not, that all children participated.

In the ANOVA, significance was found between the behaviour component and respondents' reports that it was unlikely that all children participated; $F(5,279)=6.84, p<.001$. In other words, if respondents reported that certain children consistently dominated, put their hand up, merely on-looked, or behaved disruptively, they tended to indicate that it was unlikely that all children participated. Respondents' ratings of the likelihood of all children participating at 
mat time and their agreement that children utilise differing participatory behaviours at mat time is depicted in Figure 4.16.

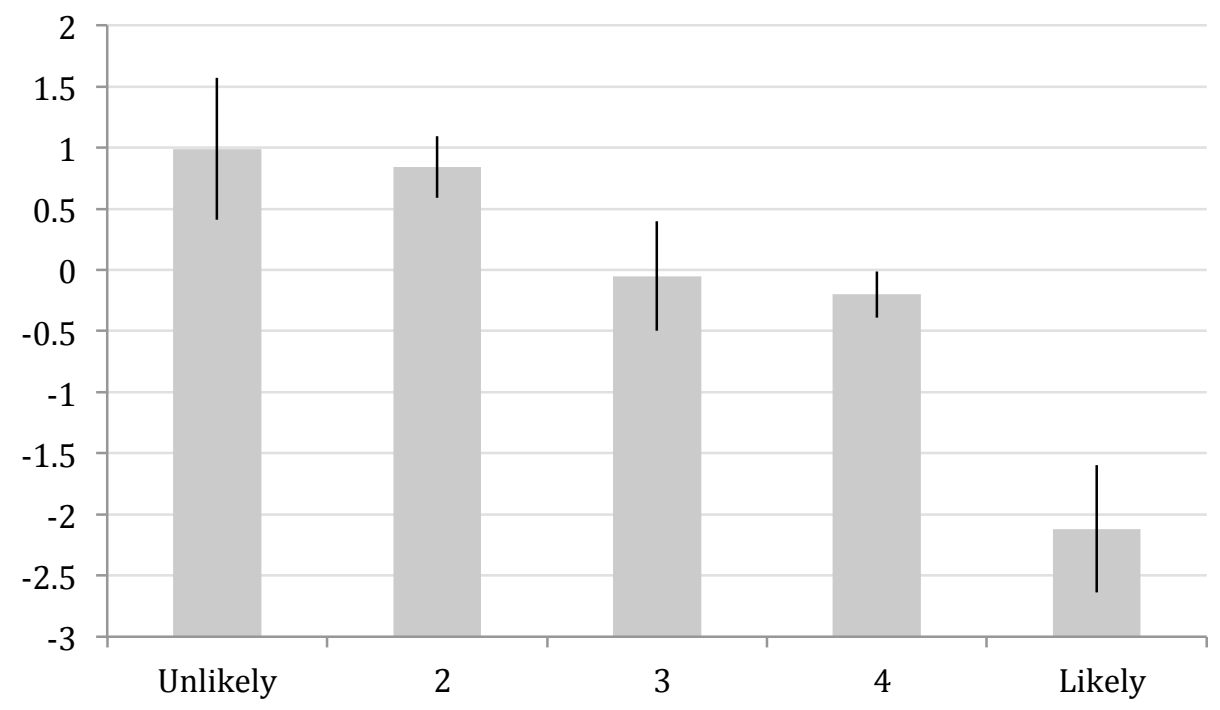

Figure 4.16. Respondents' ratings of the likelihood of all children participating at mat time and their agreement that children utilise differing participatory behaviours at mat time (some children consistently observing, dominating, disrupting, or putting their hand up).

Finally, if children enjoy mat time or are attentive to the activity occurring on the mat, it is possible that they might be more amenable to active participation. A $2 \mathrm{x}$ 2 ANOVA was conducted that used teachers' agreement of children's feelings about mat time, or not, with their agreement that all children participated at mat time, or not.

Figure 4.17 depicts the results of the $2 \times 2$ ANOVA concerning positive feelings and participation. The ANOVA indicated significance between respondents' reports of about children's positive feelings (children are attentive and enjoy mat time) and their reports on the likelihood that all children participate; $F(5$, $285)=7.33, p<.001$. If respondents indicated greater likelihood that children were attentive and enjoyed mat time, they also tended to indicate that it was likely that all children participated at mat time. 


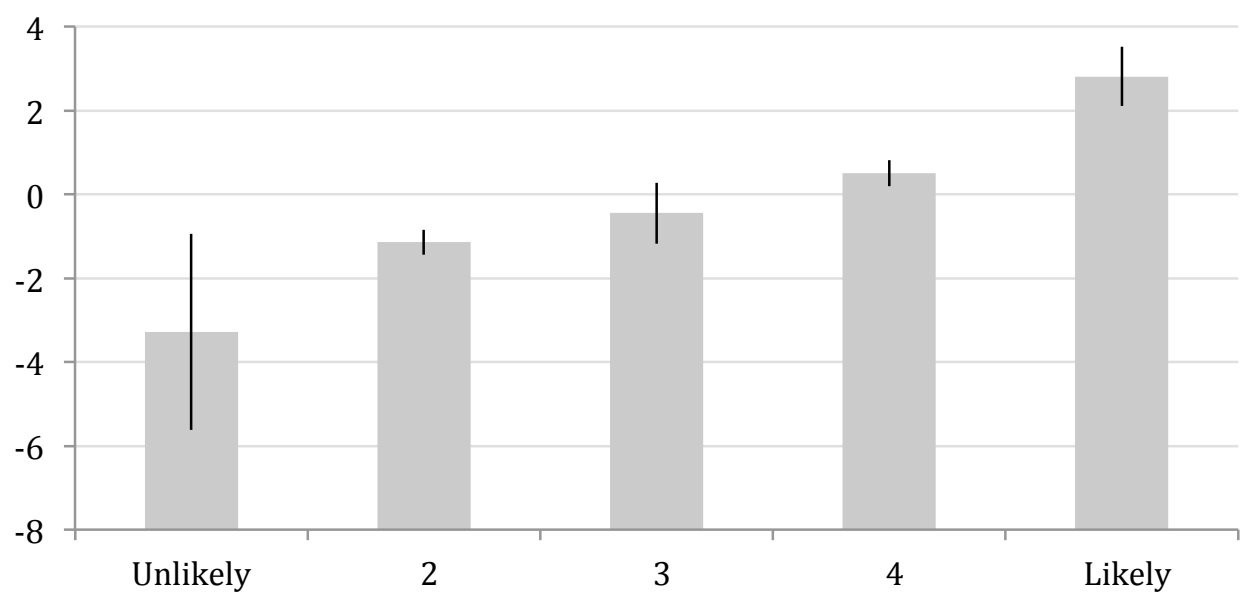

Figure 4.17. Respondents' ratings of the likelihood of all children participating at mat time and their agreement that children enjoy mat time and are attentive (positive feelings).

\subsection{Summary of findings}

The survey revealed that a significant proportion of classroom time (15-22\%) is spent on the mat for a large number of children, and the findings indicated that they are likely to experience a wide range of activities while there. These activities include those that have an academic focus, such as explaining a learning activity or teaching curriculum knowledge. In addition, mat time also includes socially oriented activities, such as welcome activities and discussion of social issues or rules. The least popular activities included music and games.

Participating teachers indicated their agreement that numerous pedagogical goals are achieved by using mat time. There was high agreement that mat time contributes to the achievement of academic aims such as children's enhanced language and listening skills, and knowledge. Similarly, teachers tended to agree that mat time contributed to social and emotional goals such as group cohesion, self-control, and improved self-esteem. 
Facets of teacher-facilitation at mat time were investigated. Previous studies (Danielewicz et al., 1996; Gallas, 1992; Murphy, 2003; Poveda, 2001) criticised high teacher control at mat time, such as rigidity of planning and teacher control of talk, which seemed to inhibit naturalistic styles of dialogue and narration. Whereas respondents in the present study indicated that their facilitation was more likely to be discursive rather than instructive, they rated their general teaching approach at mat time as slightly tending more toward teacherdirectedness, rather than child-directedness. Similarly, there was a slight tendency for the mat time to be planned (as opposed to not planned). In addition, many of the teachers indicated that they frequently ask questions during mat time.

Teachers reported considerable differences in children's behaviour at mat time. For instance, there was a high frequency of agreement that some children consistently dominated or consistently raised their hands. In contrast, teachers reported that some children took on a much less active role than their peers, in that they consistently observed rather than directly participated. Nearly half of the teachers agreed that specific children were consistently disruptive at mat time.

There was a very strong tendency for teachers to indicate that children enjoyed mat time (79\%); however, comparatively few agreed that children were attentive (18\%). Because teachers generally indicated that children were not attentive, the results were unexpected regarding teachers' agreement that children enjoyed mat time and that teachers found mat time relatively unchallenging. Rather, teachers predominantly indicated that the various pedagogical tensions were sometimes easy to manage and sometimes challenging. This is likely attributable to the scale design; a four-point scale would have given far clearer results.

Based on the literature, it was anticipated that teachers who incorporated aspects of children's interests into mat time activities might differ in their reports to those of their colleagues who do not; for example, the extant research suggests that teachers who appeal to children's interests might enhance their engagement 
(Schraw et al., 2001), improve on-task behaviour (Zaghlawan \& Ostrosky, 2011), but experience specific pedagogical tensions, such as balancing those interests with the curriculum outcomes (Alexander et al., 2008; Rubenstein Reich, 1994). The present study found that the key difference between teachers who reported that they incorporate children's interests, and those that do not, was their agreement about the likelihood that all children participate. The results indicated that teachers who reported using children's interests were more likely to report that all children participated at mat time compared with teachers who did not report use of children's interests.

The likelihood of all children's participation was explored further though correlation and ANOVA tests. In the present study, there was a weak but significant correlation between respondents' reports of the likelihood that all children participate and teaching pedagogy that was child-directed. Nonetheless, the subsequent ANOVA showed significance in that the respondents who indicated that there was likelihood of all children's participation also disagreed with statements about children's observable behaviours (such as, certain children behaved disruptively, dominated, or on-looked). The final ANOVA showed significance; respondents who agreed that children were attentive and enjoyed mat time also agreed that it was likely that all children participated at mat time.

One area in which teachers did not differ related to the degree of difficulty reported in resolving pedagogical tensions (for example, balancing children's interests with the curriculum). No differences were found between teachers who reported drawing on children's interests at mat time, and those who did not. Similarly, no differences were found between respondents' reports of their general pedagogical approach and their reports of using children's interests. Indeed, both groups were just as likely as each other to report that mat time is planned, teacher-directed, and instructive. 


\subsection{Discussion}

\subsubsection{Amount of time spent on the mat}

Respondents indicated that they typically facilitate mat times in comparatively short bursts of 10 to 20 minutes at a time. The duration of time is consistent with Mosley's (2005) recommendation for mat time being not more than twenty minutes long in order to prevent children's boredom. Nonetheless, given that the present results suggest that mat time is held several times a day, most children will spend a cumulative 1-2 hours on the mat per day.

\subsubsection{Contributions of mat time to children's learning}

A wide range of activities is utilised at mat time. It was unsurprising to see the high use of activities that align with focus on literacy given the New Zealand Government's current focus on literacy and numeracy (Clark, 2010). Similarly, activities that promote social conventions and potentially communicate differences between teachers and students were prominent, for instance, using mat time for greetings and farewells. These are symbolic events that acknowledge others as social entities and signal the level of formality expected (Firth, 1972). Similarly, discussion of rules was a highly nominated activity. This is perhaps to be expected given that, when a large group of children operate together over a day, a teacher may feel a need to maintain control, especially where she or he wants to achieve specific objectives. Moreover, forty years of research into the prevention of problem behaviour at school has emphasised the importance of teachers establishing and discussing rules and appropriate behaviours in order to reduce classroom disruption and challenging behaviour (Sugai \& Horner, 2002).

There was little indication that mat time in New Zealand regularly includes activities that previous studies have found appeal to the children, namely games and music (Cefai et al., 2013; Lown, 2002), or news (Murphy, 2003; Poveda, 2008). It is possible that music and games happen at other times (off the mat). Another activity that was infrequently used by teachers was the presentation of 
children's news. In retrospect, there could have been two interpretations of 'news' by the survey respondents. The first is morning news, when the teacher informs the children of anything important that affects their day, such as a visit from a guest speaker. The second (and intended) meaning is 'show-and-tell' or 'sharing time'. Irrespective, the extent to which children shared narratives was not effectively probed by the survey.

Respondents nominated a range of outcomes arising from mat time. They were more likely to rate improvement to children's language and listening skills than cognitive knowledge as outcomes of mat time, despite the emphasis on academically oriented activities. Other highly rated outcomes were children's social understandings and sense of group cohesion. Group cohesion in the international literature on pedagogy using the mat is poorly defined (see Cefai et al., 2013; Collins, 2013; Duman, 2009; Mary, 2014; Miller \& Moran, 2007; Rothlein et al. 1988; Rubenstein Reich, 1994). However, contemporary definitions from more general literature include a relational aspect of togetherness, as well as a shared orientation to the task at hand (Bruhn, 2009; Dion, 2000; Greer, 2012). It seems, then, that group cohesion is likely to be connected in some way to children's social understandings and ability to cooperate with each other.

Several studies have identified mat time activities that contribute to group cohesion, namely news, music, and cooperative games (Danielewicz et al., 1996; Gallas, 1992; Harmon-Jones, 2011; Mary, 2012). These activities potentially facilitate better knowledge between children about each other, thereby potentially developing greater empathy towards group members (Cefai et al, 2013; Kelly, 1999; Leach \& Lewis, 2012, Lown, 2002; Mary, 2012). Nonetheless, respondents in the present study indicated that they infrequently offered news, music, or games at mat time; therefore it is not known how mat time contributes to group cohesion. 
Despite the teachers' reports, it would be unwise to assume that mat time contributed positively to specific outcomes without knowing the content of the activities, how they have been facilitated, and the specific effect they had on children. For instance, self-esteem and group cohesion are notoriously difficult to measure and perceptions might not align with empirical data (Greer, 2012; Miller \& Moran, 2007). Notwithstanding, some researchers suggest that certain mat time activities contribute to children's self-esteem and group cohesion. However, they also contend that facilitation is essential. For instance, Svinth (2013) observed games that were so competitive that they had a detrimental impact on group cohesion, and Leach and Lewis (2012) noticed that discussion of social issues lead to bullying where some children breached the confidence of others or set them up to be told off for things they had no hand in.

\subsubsection{Pedagogies associated with the use of the mat}

Teaching approaches were slightly weighted towards planning and teacher directedness. It is of interest that the teachers in the present study indicated that they rated themselves as frequently asking questions but scored comparatively lower on facilitating mat time as a 'discussive' event. This implied that many respondents believe that there are controlled opportunities for children to answer questions and be involved in some discussion. Moreover, if teachers found one of the given potential tensions challenging, she or he was likely to find them all challenging. This finding raises two obvious possibilities; the first is that there are differences in teachers' awareness of the group of children's responses to mat time. The second possibility is that teachers have varying skills in facilitating mat time.

Teaching approaches did not systematically differ according to whether or not respondents utilised children's interests in their mat time activities; therefore, no inferences about enabling children's influence or relaxed teacher control can be made. The similarities reported here might have involved a lack of uniformity in how teachers assessed their own practices. In other words, no 'benchmark' was 
provided in the survey to indicate which practices were perceived as, say, teacher directed, as opposed to child directed.

Respondents' reports about use of interests did not appear to bear any relation to whether they reported finding the presented potential tensions challenging. One possibility that would partly account for this result was that teachers might not have been fully aware of elements of the tensions; for example, some children's needs might go unrecognised by the teacher (in which case, it presents no challenge). This is understandable, given that mat time is a socially complex event, especially in the New Zealand context in which teachers typically deal with large numbers of children on the mat. However, international researchers suggest that mat time is an area that requires teachers to reflect on their practices more objectively. Indeed, Murphy (2003) opined that teaching on the mat has become so ritualised that teachers no longer question its purpose or their own pedagogical decisions. Another final possibility is that the teachers had varying perceptions of what was meant by 'children's interests'. In retrospect, the inclusion of a definition in the survey would have been helpful.

\subsubsection{Teachers' perspectives of children's engagement and participation}

It seems possible that children's attentiveness is not a prerequisite for engagement, or even compliance (since teachers reported that attracting children's engagement and compliance are not consistent challenges, and yet also reported low levels of attentiveness). However, this seems unlikely given that explaining a learning activity or teaching curriculum knowledge were common uses. It is possible that teachers' assessments were based only on their observations of children who take an active part rather than the overall group. Further weight is given to this argument when one considers the high agreement that certain children consistently observed only. In other words, children who did not take an active role may have sat passively on the mat rather than misbehaving. Given that there were also low reports of the use of reprimands as a strategy to gain children's engagement, it seems likely that teachers may have not 
felt a need to observe children who were not taking an active role. On the other hand, the children who actively participated most likely had the teachers' attention and were also perhaps most likely to display overt signals of enjoyment.

Teachers tended to agree that children exhibit disparate participation behaviours, with their impressions typically being that certain children consistently dominate or consistently put up their hands, whereas others consistently observe only. It is likely that not all children want to speak out at mat time, nor do they all possess the same confidence or proficiency in their oral language. Nevertheless, a consequence is that children who speak often and articulately arguably have more influence over classroom practices than their less participatory peers. It is likely that such influence will be weighted towards a learning environment that better suits their own learning needs than those of other children. In other words, when specific children are allowed to dominate classroom discussion, they are indubitably replicating their own social capital. For example, the mat may be an effective forum for influencing the curriculum when a child has an opportunity to participate often, especially when it concerns sharing his or her ideas, suggestions, and interests (Kessler, 1989).

A second possible explanation of reported disparities in participation is that teachers enable varying opportunities for participation. For example, in Piter's (1995) study, the teacher tended to call on students whom she perceived to be academically competent most often, and called on other students infrequently. While the other students still participated, it was to a much lesser degree than their more esteemed peers. What is more, when a teacher reinforces a child's participation through positive feedback, the child is likely to be more positively viewed by peers (Mikami et al., 2012; White \& Kistner, 1992). As such, disparate modes of participation should be treated as an equity concern. In short, the resulting feedback arising from feedback could act as a mechanism that potentially further empowers the already powerful, and disservices those children who are already disadvantaged. A final possibility is that children's participation 
modes (gregarious or otherwise) are more dependent on other factors than the inclusion of interests, such as student temperament and self-concept or clarity of information presented by the teacher (Houser \& Frymier, 2009).

Teachers gave contrasting reports about the likelihood that all children participate at mat time. The analysis investigated whether teachers who used children's interests rated children's participation differently to those who did not. The results indicated that teachers, who used children's interests, were more likely to report that all children participated, compared to teachers who did not use children's interests. Frisby, Berger, Burchett, Herovic, and Strawser (2014) suggested that when students talked about something they knew about or had interest in, they were less fearful of making mistakes, thus increasing the likelihood of their participation. Similarly, Foster et al. (2009) stated that,

Subject matter relevant to students'... interests is one way to enhance student discussion of the subject matter. When students can see a connection between what is being discussed and issues in their own lives, they may be more likely to actively engage in class discussion. (p.174)

Either way, participation that is distributed across the group is more likely to expose children to diverse ideas (Chen \& Looi, 2011) and enhance the vitality of the class discussion (Foster, et al., 2009, p. 174). Nonetheless, using children's interests is arguably not the only mechanism for achieving this. For example, Frisby, et al., (2014) noted that when individuals feel a responsibility to share ideas, they were more likely to participate. Instilling such responsibility in children is something that classroom teachers may be able to encourage. Similarly, Weaver and Qi (2005) made a similar link between responsibility and participation in stating that, "with the consolidation of responsibility, a handful of students assume the role of active participators and discussants" (p. 571). 
The proportion of teachers reporting that specific children consistently dominate, observe, put their hands up, or act in disruptive ways was the same for those reporting incorporating children's interests and those reporting they do not do so. A very simple explanation for this might be that even when teachers incorporated children's interests, they were unable to meet all children's interests all of the time. Thus the use of interests might have had a minimal impact on whether all children were engaged or bored. However, given that teachers agreed that only certain children tended to display the particular behaviours (for example, certain children consistently observed or certain children consistently put their hand up), it is likely that the explanation is more complex. For instance, certain gregarious or circumspect behaviours might be more closely associated with a child's status or personality than whether or not a teacher has incorporated his or her interests.

Nearly half of the respondents did not indicate that they appealed to children's interests in order to engage them at mat time. We can rule out the possibility that the children were already engaged, thus ameliorating the need for extra effort by teachers, because almost $20 \%$ of the teachers reported that children were not attentive. One possibility to account for the low response rate with regard to children's interests is that as a phenomenon, interests is fairly complex. The survey did not attempt to clarify between interest residing within a learner (Mitchell, 1994), or situational interest arising from novel or attention-grabbing stimuli (Schraw et al., 2001). The lack of clarification may have deterred some participants from selecting the strategy as one that they used.

A second reason might involve the focus on curriculum required of teachers, and teachers' concern about meeting those requirements without distraction (Clark, 2010). Nonetheless, few teachers indicated that they experienced tensions between delivering the curriculum and incorporating aspects of 
children's interests into their mat time pedagogy. A third possibility relates to one very pertinent finding by Weber (2003), which is that teachers utilised those strategies that engaged children's interest in learning activities the least, especially because it involved allowing children to influence the topic and the activity. The same possibility is conceivable at mat time. Moreover, Renninger and Hidi (2011) suggest that engaging children via their interests requires a teacher's careful attunement. As already suggested, teachers might struggle to demonstrate the same degree of attunement to children when teaching them as a whole group, compared with the attunement demonstrable in one-on-one or small-group scenarios.

Further analysis was undertaken to investigate the differences between those respondents who reported that they engaged students by appealing to their interests and those who reported that they did not. Respondents were equally likely to agree that children enjoyed mat time, and that also they were not attentive, despite whether they drew on children's interests as an engagement strategy. This was a surprising finding in relation to those teachers who said that they included children's interests. Several possible reasons exist to account for the similarities in reporting with regard to enjoyment and attentiveness:

- Children enjoy mat time but are often inattentive because they are distracted by peers (see Meyer et al., 1994; Milman, 2009),

- Children enjoy mat time but are influenced by peers to focus elsewhere than the teacher; a kind of contagious distraction (Milman, 2009),

- Children enjoy the mat time but may not have been able to see relevant materials or hear the teacher adequately (see Nelson, Kohnert, Sabur, \& Shaw, 2005), and

- Children enjoy mat time but fidgeted; teachers may mistake children's fidgeting for inattentiveness.

With regard to the latter point, adults may construe fidgeting behaviour as indicative of loss of focus (Farley, Risko, \& Kingstone, 2013), yet many young 
children seem able to fidget while remaining on-task. For instance, Milman (2009) cited examples such as a child, focused on child balancing a pencil on his upper lip, who was also able to answer his teacher's questions even though the pencil appeared to absorb his attention more so than the lesson. Accordingly, Milman referred to such children as "skilled (and mysterious) multi-taskers" (p. 131). Further research is required that seeks the importance of seating position in relation to seeing and hearing well, the specific aspects of mat time being enjoyable and engaging, and finally, the influence of the peer on individual children's enjoyment and participation at mat time.

It is important to acknowledge that the survey relied on teachers' self-reports. It is, therefore, only their perceptions and opinions that are represented in the results. It is possible that some of their perceptions are not an accurate reflection of what actually happens. Teachers' constructs about children's participation and the teacher's role in managing it need to be explored further. For instance, the teachers showed high agreement that particular children dominate at mat time while others merely observe. On the other hand, they tended to disagree that encouraging all children's participation is challenging. Further investigation into these areas is warranted, especially in light of previous research indicating that active participation is important to individual children's learning. It seems possible that the peer group plays a role in the ability of individual children to actively participate (for example, see Danielewics et al. 1996). It is not known whether active participation at mat time is an important aspect of children's peer culture. Further research that asks children directly and observes their behaviour around participation is justified. 


\subsubsection{The mat as an official space and its contributions to the interactional milieu}

The present study reported that children are typically required to spend a significant amount of time on the mat, where $80 \%$ of participating teachers reported discussing rules at least once every day. Moreover, reported mat time pedagogy was slightly weighted towards teacher-directed practices. Mat time is thus likely to be somewhat formal and planned, meaning that children's contributions are likely to be carefully controlled. However, there are likely to be some opportunities for children's input at mat time, given that teachers indicated that they utilise some child-directed and flexible practices.

Many participating teachers viewed mat time is a useful forum for achieving social and academic goals. Preconceived notions about competence are likely to be promoted given that $85 \%$ of the teachers use mat time for teaching curriculum knowledge. Nevertheless, there is very little evidence that mat time is an effective forum for learning. Indeed, what little research has been undertaken, suggests that for academically vulnerable students, it is one of the least effective teaching formats (Myhill, 2002). In the present research, the data suggest that the validity of teachers' claims that mat time achieves positive social and academic outcomes is questionable. In the first instance, only an approximate $20 \%$ of teachers thought that children were actually attentive. Teachers also indicated agreement that some children behaved disruptively or merely observed instead of taking an active part.

Mat time typically features teachers' focus on asking questions of children. Previous research about questioning indicates that there is a strong probability that the IRF sequence is the predominant mode of interaction, despite it being one of the least effective for complex learning and thinking (Cazden, 2001; Myhill et al., 2006; Nassaji \& Wells, 2000). In the present study, slightly more teachers nominated children's enhanced listening skills (93\%) as something that mat time 
fostered rather than their language skills (86\%). Notwithstanding, it remains unknown what kind of communication the teachers facilitate.

The present research found that teachers predominantly characterise togetherness and a positive social climate as important facets of mat time. Children are typically welcomed and farewelled as a group. Many teachers indicated high agreement that mat time fosters group cohesion (86\%) and 65\% indicated that it enhances students' self-esteem. Furthermore, some teachers used it for feel-good actions, such as acknowledging good work (62\%). The positive climate of the mat commonly inherent in the teachers' reports is one that is friendly and enjoyable. This was evinced by the $79 \%$ of participating teachers who agreed that children enjoy mat time.

Finally, if teachers attempt to make mat time interesting to children, then they are more likely to report that all children participate. Moreover, when teachers reported that all children participated, they were more likely to report that children were attentive. Each of these points gives credence to the view that the official space at mat time should incorporate children's interests and aim to distribute opportunities to actively take part across the group.

\subsection{Chapter summary and conclusions}

Children spend a significant amount of time on the mat in New Zealand year two classrooms. The teachers participating in the survey reported facilitating a blend of social and curriculum related activities; for example, discussion of rules, and explanation of learning activities, or curriculum knowledge. Given that group cohesion, social understandings, language skills, and listening skills were also all highly rated outcomes, it seems that mat time is a significant socialisation event within a child's school day. This is an important finding that potentially relates to how teachers expect children to participate and interact with each other on the mat. 
Despite teachers' reports that mat time presents few consistent challenges to enabling all children's participation, teachers differed in their reports as to whether all children did indeed participate. Moreover, they reported high agreement that participation roles varied considerably between children. Some children were reported as actively participating in the mat time, whereas others were reported as observing. Achieving clarity about children's participation is important considering the potential link to their learning (Han et al., 2005) and influence (Emilson, 2007). This is relevant given that while there is a possibility that differences in participation might be the result of not all children having the desire to participate, some children with competitive ability may be able to enhance their own chances for participation; for example, by blocking participation for less competitive peers (Danielewicz et al., 1996).

Divergent participation patterns among children raise some philosophical questions about how participation relates to fairness and influence. For instance, does fairness require that all children have an equal number of turns at participating in certain mat time activity? Answering this question would need to take into account the possibility that some children might not want to participate as well as the quality of participation that different children are afforded. These issues relate directly to the facilitation and type of control that the teacher exhibits (Emilson, 2007). In the present study, teachers tended to place their practices on the mid-point of a range of scales to do with planning or instructiveness, indicating that there may be limited opportunities for children to exert influence.

Teachers must examine the culture of the class at mat time. Many studies have examined disparities in influence between the children and the teacher, and the impact on the nature of the children's participation (see Emilson, 2007; Ernst, 1994; Gallas, 1992; Leach \& Lewis, 2012). However, it is equally likely that disparities exist between individual children, thus impacting their ability to participate and share their unique voice (Danielewicz et al., 1996; Leach \& Lewis, 2012). Teachers' investigations of which children (1) are highly chosen by peers or teachers, (2) predominantly 
participate, and (3) are largely silent, may be essential to determine whether learning opportunities on the mat are equitably accessible to all children.

Even though teachers predominantly reported practices such as allowing some child-direction, informality, and spontaneity, there did not appear to be any bearing on whether or not they incorporated children's interests into mat time activity. Nonetheless, in the present study, teachers who reported that they considered children's interests in their pedagogy were likely to report that all of the children participated. There are potential benefits associated with participation that is distributed across the group, including diverse representation of ideas and vibrant discussion. However, regardless of whether teachers drew on children's interests, teachers were still likely to observe disparate modes of participation and found aspects of mat time sometimes challenging and sometimes easy. Therefore, using children's interests did not appear to influence whether or not teachers considered specific children to be consistently disruptive, to put their hand up, or to merely observe. 


\section{CHAPTER FIVE \\ Study Two}

\subsection{Introduction}

This study explored children's perspectives on mat time, particularly those pertaining to their feelings about it, and the extent to which they believe that their own participation is important. The primary aim was to uncover aspects of peer culture and the relatively hidden under-life of the peer group. The under-life of peer culture is characterised by unofficial scripts, symbols, and norms (see Corsaro, 1985; McLaren, 1999; Woodrow, 2006). Each of these facets potentially uphold and reproduce a teacher's official rules and expectations pertaining to children's behaviour and participation on the mat. Conversely, peer-culture scripts, symbols, and norms might counter and challenge those rules. For example, Milman's (2009) ethnography of morning meeting showed that some of the children only pretended to pay attention to the teacher. This subterfuge appeared to have a bonding effect among the children. However, irrespective of whether children's peer culture and under-life support or contravene the official rules and norms, they most likely influence children's social behaviour on the mat.

It is important to note that peer culture is not a homogenous culture. Galbraith (2011) asserted that peer groups typically consist of many sub-groups with varied internal power dynamics. These dynamics might challenge official scripts, especially those promoting equal access to participation. Specific children might require the social support of peers in order to participate in classroom activities (Danielewicz et al., 1996; Jenkins \& Kilpatrick Demaray, 2012; Sandberg \& Eriksson, 2008). For instance, it is conceivable that being helped by friends might enable some children to access more participatory roles than other less socially supported peers. Furthermore, Sandberg and Eriksson (2008) suggest that children's social support enables them to exert influence within the peer group. Accordingly, when children have a high-status position in a social hierarchy, their participatory roles might carry more influence compared with those of their less dominant peers. 


\subsection{Study aims and research questions}

Children's participation cannot be separated from social activity and the rules governing that social activity. Many studies indicate that teachers view mat time as an activity that fosters togetherness in a group. Teachers also purportedly report that mat time appeals to children's interests in different topics, and is useful for teaching and learning. Overall, teachers seem to view mat time as something that children enjoy (for examples, see Eirich, 2006; Gibbs \& Wilks, 1991; Hong, 1995). However, there are few studies that have asked young children about mat time, and those that have, suggest that young children enjoy mat time activities that entail physical movement (Cefai et al., 2013), otherwise young children demonstrate little enjoyment of mat time (Rubenstein Reich, 1994). The present study is guided by the following question:

- What are children's impressions of mat time?

Children's participation at mat time is one aspect of social behaviour, and yet it has not been a major focus in the literature about mat time. Of the studies reviewed in previous chapters, two indicated that children's participation might be important to their attentiveness. Zaghlawan and Ostrosky (2011) suggested that children's active participation might be important to their engagement, and Wood et al. (2009) found that children appeared to exhibit more on-task behaviours when they could participate by using response cards compared with a situation in which active participation was limited to a few children. However, both of these studies approached the peer group as a single unit, and therefore the internal dynamics that are relevant to participation, such as social hierarchies and peer-affiliations, were not addressed. Given the paucity of research, this second study aimed to address two questions:

- What importance do children place on their own participation and engagement at mat time?

- How do children's peer relations influence their participation at mat time? 


\subsection{Methodology}

This study used a pragmatic approach (as described by Johnson \& Onwuegbuzie, 2004). Such an approach rejects both reductionism and dualism; for instance, the presentation of subjectivism and objectivism as discrete polarities. It recognises the existence of the inner world of subjects as well as the existence of objective reality. Such an approach can offer a multi-lens account that describes and explores a phenomenon by using the explanations given by study participants. In turn a researcher makes sense of those explanations (Johnson \& Christenson, 2004).

Data were gathered through semi-structured interviews. In a semi-structured interview, questions are posed in an order that suits the flow of the interview. Additional questions are also added when required, so that the researcher can achieve a clear understanding of what the participants want to convey. The interviewer has a clear agenda regarding the topic, but allows the participant to elaborate on points of interest. The interviewer must know about the subject being discussed and be able to identify new information that arises from the interview and ask further questions about it. As such, semi-structured interviews often engender data that have specific foci; yet they also allow participants to share the qualitative nuances of their lived experiences and beliefs (Anderson, 1999; Cresswell, 1994). Specifically, the raw data that were generated through the semi-structured interviews were qualitative. The analysis comprised of thematic development, scrutiny, and synthesis (see Johnson \& Onwuegbuzie, 2004).

\subsubsection{Ethics}

The Victoria University of Wellington Faculty of Education Human Ethics Committee granted ethical approval to conduct the study (ref number: SEPP/2010/115 RM18188, see Appendix B). Ethical considerations determined many of the decisions about research processes in the present study. I developed a list of guiding principles that underpinned the study; these were that: 
1. The adults who knew the children would be consulted first.

2. Children had the right to give assent without coercion, or deny participation without repercussion, at any stage of the research process.

3. Children's learning would not be unreasonably interrupted.

4. Children's anonymity would be protected at all times.

5. Children's emotional safety was paramount during all steps of the research.

6. Children's assistance in the study would be acknowledged.

In order to be part of the study, a rigorous programme that sought adults' consent was undertaken. Information letters were disseminated to the three school principals, the teachers, and the children's parents. Their written consent to the study was required before proceeding to seek assent from the children. The children were also given a letter and written assent form. The letters are provided in Appendix C.

I took great care to treat every child similarly, irrespective of whether they had given assent or not. Any favouritism could have acted as a coercive element. Steps to avoid coercion were taken at each interview and the child was verbally assured that they could terminate the interview at any time without repercussion, and that there were no incorrect answers; rather, it was their opinion that was being sought. In addition, I explained how the information they shared would be used.

To minimise disruption to children's learning, the teacher was given control over which assenting children could be interviewed and when. Moreover, the actual interviews took approximately ten minutes, which meant that each child was away from lessons for a minimal amount of time. Children were given assurance of their anonymity. To this end, their names were removed from all data and replaced with codes. For the purposes of reporting, pseudonyms have been used. 
Finally, care has been taken to remove any identifying features from descriptions of participant classrooms, teachers, schools, or children.

Children's emotional safety was a key consideration at all times. I spent a minimum of a term engaged in each class, visiting at least once a week and up to three times a week. The first visits were to build relationships. Prior to the interviews, mat time was video-recorded for use in validating findings ${ }^{6}$, and I often sat on the mat with children. Interviews occurred toward the end of the video-recording phase, allowing me to establish knowledge of the children and form relationships with them prior to interviewing them. In addition, I am a trained and registered early childhood teacher. My prior experience enabled me to perceive emotional discomfort expressed by children during the research process. Even when children had given their assent to be interviewed, if they appeared to be uncomfortable or anxious during the interview, I terminated the interview immediately and reassured the child. This occurred on two occasions with children, who teachers later told me, were prone to anxiety.

Finally, given that I forged relationships with the children, my transition out of the classrooms at the completion of the research was planned. I frequently talked to children about the 'last visit' throughout the data-gathering period. At the conclusion of the data collection, children and teachers were given small gifts as acknowledgements for their participation and for hosting me in the classroom settings. Every child received a gift regardless of whether they were interviewed or not. Gifts were chosen based on my understanding of the class. For example, in one classroom, every child was given a sheet of stickers, whereas in another, each child received a small gift bag of erasers. Children were not aware of the gift until my last day in the classroom to avoid it influencing children's decision about participation.

${ }^{6}$ The findings of the video-observations are not reported in the present thesis. 
Ethical considerations were also made in relation to the handling of data. The person employed to transcribe the interviews was required to sign a confidentiality agreement (see Appendix D). Before the transcripts were handed to a reliability-rater, the children's and teachers' names were removed and replaced with codes. Any other identifying information was also removed. Data were stored on a computer requiring a password, and printed copies of data were kept in a locked filing cabinet in my office at Victoria University of Wellington. In addition, the data were backed up on an external hard-drive, which was similarly locked up.

Another ethical aspect of research with children that has particular importance in the New Zealand context is conducting studies in ways that situate children as members of their family and wider community (Te Maro, 2010; Te One, 2010). In this present study, the teachers acted as agents, facilitating communication between the children's families and myself, in that they conducted discussions with families and fielded questions after completing an initial information session with me. This might be recognised as a breach of cultural protocol in the New Zealand context, where it is the researchers who should address any concerns about the research and the research process by meeting with families and community directly (Te Maro, 2010).

\subsubsection{Selection of classrooms}

In the previous chapters, it was explained that a survey was administered to teachers of year two children in New Zealand. Incorporated in the survey was an invitation for teachers to volunteer their classes for this second, more in-depth study. The response to this invitation was high, with 150 teachers indicating an interest. Given that interviews with children were face to face, it was most convenient to narrow the participating classrooms to the greater Wellington Region, where I reside. Fifteen of the teachers who volunteered their classes lived in the Wellington region. Each of these teachers were emailed and asked to provide a brief description of their class and their mat time practices. In addition, they were sent an information letter about the study. Five full responses were 
returned. From these, three classrooms were purposively selected, based on maximum variation sampling (Wiersma \& Jurs, 2009); in other words, the three classrooms that were chosen were those that appeared to have the most points of difference to each other. These were based on class size, decile ${ }^{7}$, children's ethnicities, and mat time pedagogies. The three participating classes were labeled Classroom 'A', 'B', and 'C'.

Classroom A was in a decile 10 school. It was small, with fewer than twenty children. The children descended from a range of European and Asian cultures. Mat time was held several times each day as an introduction to specific skills or activities, to engage in class discussion, games, or for social reasons (for example, 'news'). Children gathered on a spacious mat at the front of the class in a freeseating fashion. The teacher sat on a chair in front of them. The classroom was well resourced with electronic equipment, such as Vimeo whiteboards, laptops and data projectors. These were often used in various educational games or activities. Nine children were interviewed.

Classroom B was exceptionally large, with over thirty children of European, Pacific, Asian and Middle Eastern descent. The class was situated in a Decile 10 school. The teacher facilitated several mat times per day, often before breaks or activities. Frequently, mat time focused on a specific subject area, such as mathematics; however, it was also used for other purposes including reading large books or playing games. Children sat on the mat in an en bloc fashion with the teacher at the front on a chair. The mat was surrounded by equipment and shelving on two sides. A standard whiteboard was utilised. A data projector and laptop computer were available if required. Twenty-five children were interviewed.

${ }^{7}$ Decile: a school's decile rating in New Zealand determines what degree of funding that school receives. Census data is used to determine the extent to which that school's community resides in low socioeconomic communities. A low decile rating is allocated to schools located in low socioeconomic communities (Ministry of Education, 2015). 
Classroom C was mid-sized, with approximately twenty children. The school was decile 1 at the time of data gathering. The children were predominantly from Māori and Pacific families. The teacher had been trying to reduce the number of mat times per day and had also moved to using a circle configuration when the children sat on the mat. Mat times were held for both academic and recreational or social purposes. Discussion was a frequent activity and the teacher also read novels to the children, or played games. The teacher sat on the floor with the children. Often she wrote on a large piece of paper that could be displayed at a later point, or used a stand-free whiteboard as opposed to one attached to the wall. The mat was relatively spacious with no other equipment nearby. Sixteen children were interviewed.

\subsubsection{Selection of participants}

Overall, 49 children were interviewed for the study. Children in the participating classes decided whether they wanted to participate. First, I held a meeting with each teacher to explain and discuss the project. Next, an invitation was issued to parents via an information letter. A similar invitation was given to children, which was brief, written in large font, and used simple language that children were likely to be able to read (see Appendix B). The teachers distributed the letter in class time and read the letter with the children. Each teacher allowed time for discussion about the project. I was not present during the discussion or when the teacher distributed and gathered assent forms.

Children were given a form that read:

Please tick the box that you agree with.

$\square$ I feel okay if Anita talks to me about mat time

$\square$ I don't feel okay if Anita talks to me about mat time 
The children were asked to make a mark in the relevant box, and the forms were gathered by the teachers and returned to me. In addition, I confirmed children's willingness to participate with them verbally, prior to each interview.

The decision to directly ask children about mat time was influenced by a belief that children are experts about their own lives. They have much to contribute to knowledge about childhood and education when asked about their experiences. Such a belief is justified by other studies that have sought children's viewpoints; for example, Te One (2010) asserts that studies that seek children's views can "deepen understanding (for researchers, teachers and children) of how classrooms are culturally constructed" (p. 67).

\subsubsection{Development of the semi-structured interview schedule and procedures}

The interview schedule was developed from the literature about mat time. First, children were asked to set up a mat time and to indicate where they sat, and explain why they sat there. This was designed to engage children's interest in the interview process and to put them at ease. Also, for this purpose, colourful, human-like figures, which are illustrated in Figure 5.1, were used to assist children in setting up a model of the mat time. Green, Cillessen, Rechis, Patterson and Hughes (2008) used toys and figures in their interviews with children, allowing them to manipulate the props. They noted that: "because young children are particularly motivated by hands-on activities, this was an effective way to engage them in the task" (p. 98). 


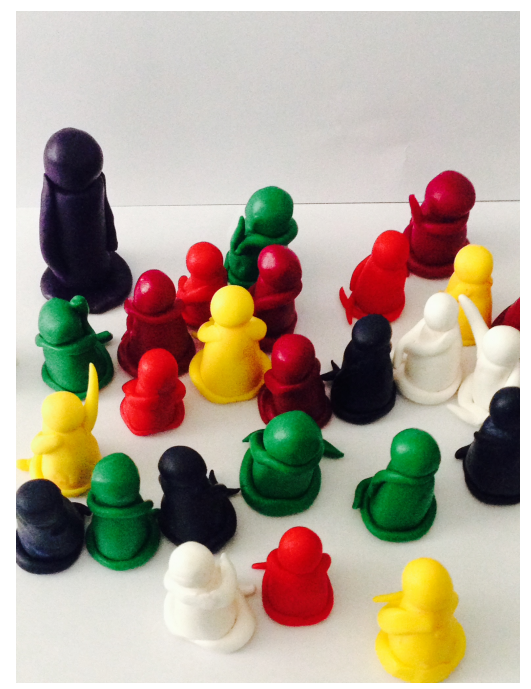

Figure 5.1. Human-like figures used as props in the interviews.

Children were asked about the best place to sit. This question was based on the assumption that children are deliberate in their choice of seating position. It aimed to uncover children's motivation as academically or socially related; in addition, it also allowed children to indicate other reasons that I had not considered. In any case, I speculated that seating position might have a bearing on children's participation, given that Mandel Morrow and Smith (1990) noted that children at the back of the mat appeared to experience greater difficulty in securing active participation than those who sat near the front.

Children were asked, 'what happens at mat time?' Eirich's (2006) work influenced the question; her ethnographic study about morning meeting found that the formation of community was an important official principle; however, she also suggested that specific children were competitive in promoting their own needs above those of others. Mat time in New Zealand differs from morning meeting; whereas morning meeting is a ritual designed to welcome children, mat time often has a stronger focus on teaching and learning. Nonetheless, Eirich (2006) was able to examine some of the official and unofficial scripts. A second article, that influenced the approach taken, was that of Leach and Lewis (2012), who had success in asking children "What sorts of things do you do in circle time?" (p. 46). 
It was anticipated that asking children about what happens at mat time or the types of things that they did, would enable them to discuss the official scripts of the mat time in terms of activities and rules. However, it also provided an opportunity for children to disclose other, relevant issues that might be less obvious or part of an unofficial script.

Another question asked children to indicate how they felt about mat time. To date, there have been mixed findings regarding children's enjoyment. Some studies have reported that children predominantly enjoy mat time (Collins, 2013; Lown, 2002; Moss \& Wilson, 1998), whereas others have reported that children seemed bored, or that they only liked specific aspects of mat time (Cefai et al., 2013; Rubenstein Reich, 1994; Zaghlawan \& Ostrosky, 2011). In this study, children were asked to rate their feelings or enjoyment of mat time using three cards depicting happy, unhappy, and neutral. The cards are depicted in Figure 5.2, and were provided to assist the children's formulation of answers. In addition, the children were asked to qualify their rating. Leach and Lewis (2012) were able to gather rich information from children about what they liked and disliked.
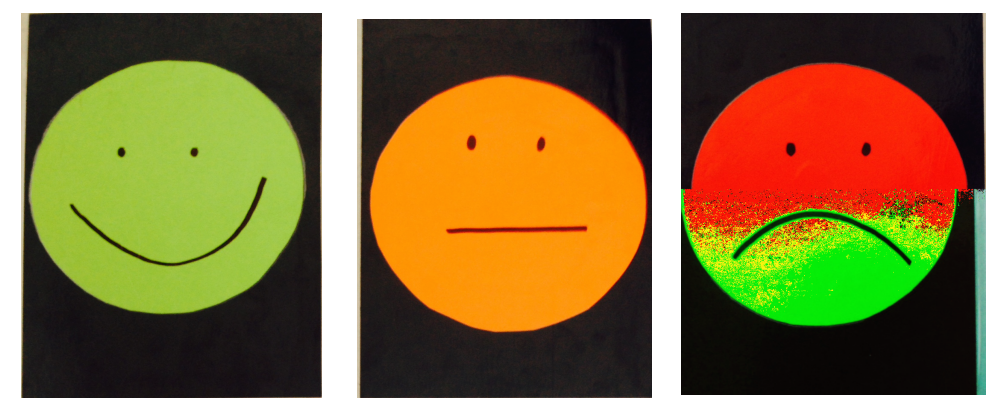

Figure 5.2. Expression cards used as props in the interviews.

The next question was about the extent to which children considered their personal participation at mat time as being important. As discussed previously, children engage in a range of participatory roles at mat time. Some studies have suggested that some children might be bored, and thus withdraw their attentiveness (for example, Zaghlawan \& Ostrosky, 2011), others suggest that 
certain children do not possess the self-regulation skills to participate constructively (Tominey \& McLelland, 2011), and others posit that teachers call on some children to participate disproportionately (Piters, 1995). However, none of these researchers have asked children about their participation directly. Finally, it was anticipated that there could be additional individual or peer concerns that might not be raised through administering the previous questions; therefore, children were asked if there was anything else that they would like to say about mat time.

\subsubsection{Piloting the interview}

The interview schedule was pilot tested with one of the children. Initially, it was anticipated that children would be asked to describe their ideal mat time. During pilot testing, the children contributed humorous responses to this question, such as, "I'd give them all chocolate". Analysis of the pilot interview indicated that the responses to the question, while delightful, failed to contribute meaningfully to the study. The question was dropped for the subsequent interviews in order to minimise the duration that each child was away from lessons while being interviewed.

Another aim of the pilot study was to test the efficacy of the props used in the interview. Te One (2010) notes that when asked, child participants of various studies expressed an opinion that the research tools ought to be "fun" (p. 75). It was true that whether the materials were engaging or not appeared to be a concern for the child in the pilot study. Originally, the human-like figures were labelled with each child's name. However, during the pilot interview, the child indicated that she wanted her name to be on another figure. Additionally, she was perturbed that none of the figures had their hands raised. To prevent participants from being distracted by such concerns in subsequent interviews, name labels were not included on the figurines, and several figures were included that depicted children with raised hands. It should be noted that the figures with raised hands did not represent any specific child. 


\subsubsection{Gathering the data}

I conducted all of the interviews. This necessitated attending each classroom for approximately half a day, at least once a week, for approximately a school term. Rather than timetabling each interview, they occurred in an ad hoc fashion, in that the teacher called on individual children, who had given their written assent, to be interviewed once the child's work had been completed to a satisfactory standard. Each teacher nominated the place in which the interviews were to be conducted. In classroom A, this was a resource room that was attached to the classroom. The teacher in classroom B made the staffroom available, and in classroom C, interviews took place in the book corner. In each setting, the children and I were not audible to others, but were visible at all times.

Prior to each interview, I set up the props so that the figurines were mixed together in a box and the cards were stacked together. In addition, paper and pens were provided in order to record the child's placement of the figurines. In two of the classrooms, the props were set up on the floor; however, for the interviews in the staffroom, the props were set up on a low-lying coffee table. The child was invited to sit opposite me, with the props in the middle. This enabled me to monitor the child's expressions and other body language while speaking.

The children were taken individually to the interview site, where I explained the study, their right to anonymity, and how the data would be reported. In addition, each child was given an opportunity to ask any questions about the study. This was important as the initial discussions about the study had occurred several months prior and children might have forgotten about these details. For several children, my role and motivations were primary considerations. For example, one child asked, "well, firstly, are you going to take this information and do you want to be a teacher?" In contrast, other children had questions about the props such as, "how did you make all these?" Each of the children's questions was answered and they were asked if they were willing to proceed to the interview. 
The interviews were audio recorded to provide a more complete representation of interviewees' discourses. Each interview was approximately ten minutes in duration. Directly prior to the interview, children were shown the dictaphone and were told that its use allowed the interviewer to attend more fully to the interchange rather than be concerned with note taking (see Henn, Weinstein, \& Foard, 2009). The children were shown the pause button and told that they could stop recording at any time. The dictaphone was then placed directly in front of them so that it was within their reach. When children wanted to experiment with turning the dictaphone off and on before the interview, this was allowed.

If the participants experienced difficulty in answering any of the questions, Denscombe's (2007) interview prompts, probes and check tactics were used. Prompts clarify the question for the interviewee and assist them to answer. Nevertheless, the prompt must not unduly influence the interviewee to answer in a particular way. Examples of the prompts used when a child seemed uncertain of how to respond to being asked about what happens on the mat is as follows:

- What kinds of activities do you do?

- Do children say or do anything in particular?

- Does the teacher say or do anything in particular?

Probes were used to elicit further information from interviewees, and included me asking for an example, more details, or clarification. Checks involved me providing a summary of understanding of what an interviewee has said. For instance, Denscombe (2007) suggested that the researcher could say, "So, if I understand you correctly..." or "What this means is that..." (p. 192). Checks enable the interviewee to corroborate or correct the researcher's understanding. In addition, Brown's (2006) prompts to non-verbal cues were taken into consideration. This involved making descriptive and non-evaluative statements, for example: "You smiled when I asked you that question" (p. 196). This strategy appears often to elicit richer data from respondents (Brown, 2006). At the 
conclusion of the interview, the child was thanked and was returned to the teacher.

\subsubsection{Analysis}

Once the interviews had concluded, I, along with an assistant to the Victoria University of Wellington Jessie Hetherington Centre, transcribed the interviews. The transcription requirements were that all speech was represented accurately, including grammatical errors, stuttering, and speech fillers, such as 'um' and 'ah'. I audited five randomly selected transcripts against the audio recording of the interview to ensure accuracy. Once the transcripts had been completed, analysis was undertaken.

An iterative approach was used in analysis, under which the researcher assumes very little about patterns or themes until the data can be viewed as a whole (Henn et al., 2009). The researcher must then construct meaning from the data, and identify dominant trends in the participants' responses (Wiersma \& Jurs, 2009). The transcripts were read and tentative categories for analysis were developed for each interview question, as well as general themes that arose across the questions. This process was iterative in the sense that relationships between categories and themes were reviewed and considered at each successive reading of the transcripts. At times this meant that certain categories and themes could be merged, and at other times, new categories or themes were required.

More specifically, a sorting and grouping technique was used that involved similar quotes from each interview being colour coded and grouped together, without being removed from the wider interviews. In other words, several paper copies of entire interviews were used in the development of each category and theme. For example, all references to friendship were found and coloured green. All interviews with green highlighting were then analysed concurrently to determine commonalities of quotes across the interviews, and the reason for those commonalities. The commonalities were labelled, creating the themes and 
categories. At the same time, polarised stances among participants, or outliers to themes and categories were recorded. Once all of the themes and categories had been identified, intra-theme and categorical analysis occurred. For instance, the theme friendship, was differentiated into the subthemes of cooperation and conflict. This process continued until no new themes or categories could be identified. The entire process was iterative, and went through many cycles.

When the categories intended for percentage analysis had coherence, inter-rater reliability testing was conducted for those categories associated with the children's desired seating position, beliefs about participation, and feelings about mat time. These categories were chosen because they appeared to be the predominant topics emerging from the interviews. The rater was a postgraduate student at the school of education, who was selected on the basis of her prior experience in research, and familiarity with education theory and peer relations theory. She attended an initial training session for an hour, where she was given a list of descriptors for the categories, and a form to record her ratings on (see Appendix E for coding instructions). In addition, she was given fifteen (30\%) of the interview transcripts to code.

A consensus-estimate approach to inter-rater reliability was used to quantify the data. The most common approach is percentage agreement, with a general guideline that $80 \%$ agreement constitutes acceptable reliability. Stemler and Tsai (2008) suggest that percentage agreement is useful in establishing the validity of the identified constructs:

Independent raters can at least reliably classify objects according to how well they meet assumptions of the construct...if a researcher is able to demonstrate that independent parties can reliably rate objects along the continuum of the construct, this provides good objective evidence for the existence of the construct. (p.30) 
We each made a total of 129 nominations with 118 identical nominations, giving an agreement rate of $91 \%$. This meant that the categories were reliable.

When inter-rater coding was complete, I conducted a second meeting with the rater. At this meeting, the rater had an opportunity to discuss other categories or themes that she had noticed, and which were not represented on the coding form. The rater indicated that she found it surprising that a high number of children said that it was more important to try, than necessarily be correct, when answering questions that the teacher posed. On the basis of this feedback, further analysis about the importance of being correct was undertaken.

\subsubsection{Trustworthiness and ecological validity}

A key consideration in relation to interviews is the threat of reactivity to ecological validity, or credibility. 'Reactivity' is the phenomenon of people acting in nonauthentic or atypical ways due to their awareness of being studied (Kawulich, 2005). To counteract potential reactivity, time was spent in each classroom forming relationships with both child and adult participants before interviewing commenced. This was particularly important in regard to research with children because of the power differential between them and adults; such power differences might lead children to say things in order to please adults, rather than saying what they really think (Liamputtong, 2007; Te One, 2010).

To mitigate the power differentials between children and adults, I used Corsaro's (1985) strategy of presenting as a non-adult. While I did not pretend to be a child, I avoided, as much as possible, acting with the authority usually afforded to adults in classroom settings. Moreover, I aimed for interactions with the children that were friendly and playful (see Freeman \& Mathison, 2009). Presenting as a non-adult was assisted by my physical size; for instance, one of the children noted that I was "not like a real adult", given that I fitted easily on the child-sized chairs. 
The quality of relationships between the children and me was also important for the credibility of data. For instance, when I issued checks of the data within the interviews, it was essential that children felt that they could disagree with me if I had made incorrect assumptions or misunderstood their intent. Evidence of children making such clarifications was evident in the interviews. An example follows:

Interviewer: So you're saying that if you're giving an answer, it's important that you get the answer right?

Child: Ummm. Aw. Nah. Nah. 'Cause it's not, you don't need to get the answer right. It's just like it would be good if you try.

Finally, my own reflexivity was acknowledged throughout the research process with the use of regular discussions with the research supervisors. My values and biases were probed and reflected on in order to scrutinise the confirmability and credibility of the findings (see Stake, 1995; Kawulich, 2007). My interpretations of the interviewees' intended meaning and the robustness of the themes were regularly challenged and adapted as a result of conversations with the research supervisors. Finally, interviews with classroom teachers and videoed observations of mat time were used as final validity measures. For example, my understanding of several children's interviews was that specific peers were often excluded. The teachers' interviews and video footage were able to confirm the accuracy of this understanding.

\subsection{Results}

As a warm-up task, the children were asked 'where do you sit?' In addition, they were asked to set up the mat time with small figurines. Neither of these questions is reported in the present section, owing to the intended purpose being to relax the children. In addition, there was considerable doubt over whether children did actually sit where they had indicated. Nevertheless, it should be noted that the children included quite a bit of discourse during the activity, such as putting their 
favourite friend close by, or placing the figurine that represented himself or herself, away from undesired peers.

\subsubsection{Seating position}

One interview question asked children to nominate the best place to sit on the mat. Because the three classrooms used different seating configurations, the results for each classroom are reported separately. Some children chose more than one position; therefore, the percentages do not add to $100 \%$. Each figure reported represents the percentage of respondents nominating a given position.

In classrooms $\mathrm{A}$ and $\mathrm{B}$, which used en bloc configurations, a distinct pattern emerged where nearly all of the children felt that the front of the mat was the most desirable place to sit (97\%). Not only that, but every child also chose the central position at the front. In other words, they selected the place directly in front of the teacher as opposed to the sides of the mat. A few children chose other positions, with $18 \%$ nominating the middle and $6 \%$ choosing the back of the mat. One child stated that she had no preference for an actual position but desired to sit wherever her close friend was, which, as it turned out, was the front.

Children's preferences for a particular position on the mat were more evenly distributed in Class $\mathrm{C}$, in which children were seated in a circle at mat time. Specifically, $47 \%$ nominated their preferred position as being next to the teacher, $47 \%$ indicated seats in the part of the circle opposite the teacher, and $27 \%$ said that the 'sides' of the circle were their preferred places to sit.

There were various considerations that children in all three classrooms used to evaluate the desirability of various positions, and these are depicted in Table 5.1. 
Table 5.1. Coding of Children's Reports on Reasons for Seating Position Preferences

Classroom A See/hear Focus Noticeability Confidence Friends

\begin{tabular}{|c|c|c|c|c|c|c|}
\hline Ella & $\mathrm{X}$ & & & & & \\
\hline Ava & $\mathrm{X}$ & & & & & \\
\hline Paige & $\mathrm{X}$ & & & & & \\
\hline $\mathrm{Nic}$ & $\mathrm{X}$ & & & & & \\
\hline Jasmine & & & & & & $\mathrm{X}$ \\
\hline Jesse & $\mathrm{X}$ & & & & & $\mathrm{X}$ \\
\hline Alex & $\mathrm{X}$ & & & & & \\
\hline Cameron & $\mathrm{X}$ & & & & & \\
\hline Keira & $\mathrm{x}$ & & & & & \\
\hline \multicolumn{7}{|c|}{ Classroom B } \\
\hline Sienna & $\mathrm{X}$ & $\mathrm{X}$ & & & $\mathrm{X}$ & \\
\hline Holly & $X$ & & & & & \\
\hline Maia & $\mathrm{X}$ & & & & & \\
\hline Rua & $\mathrm{X}$ & $\mathrm{X}$ & $\mathrm{X}$ & & $\mathrm{X}$ & \\
\hline Hunter & $X$ & & $\mathrm{X}$ & & & \\
\hline Connor & $\mathrm{X}$ & $\mathrm{X}$ & & & $\mathrm{X}$ & \\
\hline Cooper & & & & & $\mathrm{X}$ & \\
\hline Fetu & $\mathrm{X}$ & & & $\mathrm{X}$ & & \\
\hline Olivia & $\mathrm{X}$ & $\mathrm{X}$ & & & & \\
\hline Chloe & & & & $\mathrm{X}$ & $\mathrm{X}$ & \\
\hline Zoe & & & $\mathrm{X}$ & & & \\
\hline Quentin & $\mathrm{X}$ & & & & $\mathrm{X}$ & $\mathrm{X}$ \\
\hline Loto & $\mathrm{X}$ & & & & $\mathrm{X}$ & \\
\hline Tane & $X$ & $\mathrm{X}$ & & & & \\
\hline Trevor & $X$ & & & & $\mathrm{X}$ & \\
\hline \multicolumn{7}{|l|}{ Masina } \\
\hline Zara & $\mathrm{X}$ & & & & & $\mathrm{X}$ \\
\hline Amelia & $X$ & $\mathrm{X}$ & & & $\mathrm{X}$ & \\
\hline Sefa & & & $\mathrm{X}$ & & & \\
\hline Mia & $\mathrm{X}$ & $\mathrm{X}$ & & & & \\
\hline Sean & $X$ & $\mathrm{X}$ & & & & \\
\hline Luca & $\mathrm{X}$ & & & & & \\
\hline Logan & $\mathrm{X}$ & & & & & \\
\hline Simon & $X$ & & & & & \\
\hline Levi & & & & & & $\mathrm{X}$ \\
\hline \multicolumn{7}{|c|}{ Classroom C } \\
\hline Arana & $\mathrm{X}$ & $\mathrm{X}$ & $\mathrm{X}$ & & $\mathrm{X}$ & \\
\hline Isaac & & & $X$ & & & \\
\hline Rawiri & $\mathrm{X}$ & & & & & \\
\hline Anahera & & & & & & $\mathrm{X}$ \\
\hline Hana & & $\mathrm{X}$ & & $\mathrm{X}$ & & \\
\hline Hauku & & & $\mathrm{X}$ & & & \\
\hline Areta & & & & $\mathrm{X}$ & $\mathrm{X}$ & \\
\hline Hayley & $\mathrm{X}$ & & & & & \\
\hline Marama & & $\mathrm{X}$ & $\mathrm{X}$ & & & \\
\hline Peter & $\mathrm{X}$ & $\mathrm{X}$ & & & & \\
\hline \multicolumn{7}{|l|}{ Ruru } \\
\hline Matiu & & & & & $\mathrm{X}$ & \\
\hline Jack & $\mathrm{X}$ & & $\mathrm{X}$ & & $\mathrm{X}$ & \\
\hline Benjamin & & $\mathrm{X}$ & $\mathrm{X}$ & & $\mathrm{X}$ & \\
\hline Whina & & $\mathrm{X}$ & $\mathrm{X}$ & $\mathrm{X}$ & $\mathrm{X}$ & $\mathrm{X}$ \\
\hline
\end{tabular}


The most frequently nominated included those positions that enabled them to see better (36\%), or to sit with friends (30\%). Other reasons were that certain positions allowed better focus (28\%), hearing (26\%), or to be more noticeable to the teacher (22\%). Other positions enabled the children to feel more confident $(10 \%)$ or comfortable than other positions (14\%). None of the children in Classroom C nominated 'hearing better' as a reason for their selected seating preferences (although some children did nominate 'seeing better'). This classroom utilised the circular configuration at mat time and had a slightly smaller group attending the mat, which probably explains this finding.

\subsubsection{What usually happens at mat time?}

Children were asked to describe what usually happens at mat time. They mostly spoke in relation to three main activities: whole group discussion (question/answer), small group work, and competitive games involving the entire class. In addition, many of the children discussed various actions of the teacher on the mat as can be seen in Table 5.2. A high number of children identified maintaining rules (76\%), and explaining processes of activities or concepts (65\%). Finally, several children inferred that their teacher helped children in a variety of ways (30\%). 
Table 5.2 Coding of Children's Reports about What Happens at Mat Time

\begin{tabular}{|c|c|c|c|}
\hline Classroom A & Rules & Processes & Assistance \\
\hline Ella & & $\mathrm{X}$ & \\
\hline Ava & $\mathrm{X}$ & & \\
\hline Paige & $\mathrm{X}$ & $\mathrm{X}$ & $\mathrm{X}$ \\
\hline $\mathrm{Nic}$ & & $\mathrm{X}$ & \\
\hline Jasmine & $\mathrm{X}$ & $\mathrm{X}$ & $\mathrm{X}$ \\
\hline Jesse & & $\mathrm{X}$ & \\
\hline Alex & $\mathrm{X}$ & $\mathrm{X}$ & \\
\hline Cameron & $\mathrm{X}$ & & \\
\hline Keira & & $\mathrm{X}$ & \\
\hline \multicolumn{4}{|l|}{ Classroom B } \\
\hline Sienna & $\mathrm{X}$ & & \\
\hline Holly & $\mathrm{X}$ & $\mathrm{X}$ & $\mathrm{X}$ \\
\hline Maia & $\mathrm{X}$ & & \\
\hline Rua & $\mathrm{X}$ & $\mathrm{X}$ & \\
\hline Hunter & $\mathrm{X}$ & $\mathrm{X}$ & $\mathrm{X}$ \\
\hline Connor & $\mathrm{X}$ & $\mathrm{X}$ & \\
\hline Cooper & $\mathrm{X}$ & $\mathrm{X}$ & $\mathrm{X}$ \\
\hline Fetu & $\mathrm{X}$ & $\mathrm{X}$ & \\
\hline Olivia & $\mathrm{X}$ & $\mathrm{X}$ & $\mathrm{X}$ \\
\hline \multicolumn{4}{|l|}{ Chloe } \\
\hline Zoe & $\mathrm{X}$ & $\mathrm{X}$ & \\
\hline Quentin & $\mathrm{X}$ & $\mathrm{X}$ & \\
\hline Loto & $\mathrm{X}$ & & \\
\hline Tane & $\mathrm{X}$ & & $\mathrm{X}$ \\
\hline Trevor & $\mathrm{X}$ & & \\
\hline \multicolumn{4}{|l|}{ Masina } \\
\hline Zara & $\mathrm{X}$ & & $\mathrm{X}$ \\
\hline Amelia & $\mathrm{X}$ & $\mathrm{X}$ & \\
\hline Sefa & $\mathrm{X}$ & $\mathrm{X}$ & \\
\hline Mia & $\mathrm{X}$ & & \\
\hline Sean & $\mathrm{X}$ & $\mathrm{X}$ & $\mathrm{X}$ \\
\hline Luca & $\mathrm{X}$ & & \\
\hline Logan & $\mathrm{X}$ & & \\
\hline Simon & $\mathrm{X}$ & & \\
\hline Levi & & $\mathrm{X}$ & \\
\hline \multicolumn{4}{|l|}{ Classroom C } \\
\hline Arana & $\mathrm{X}$ & $\mathrm{X}$ & $\mathrm{X}$ \\
\hline Isaac & $\mathrm{X}$ & $\mathrm{X}$ & $\mathrm{X}$ \\
\hline Rawiri & $\mathrm{X}$ & $\mathrm{X}$ & \\
\hline Anahera & $\mathrm{X}$ & $\mathrm{X}$ & \\
\hline Hana & $\mathrm{X}$ & $\mathrm{X}$ & $\mathrm{X}$ \\
\hline Hauku & $\mathrm{X}$ & & \\
\hline Areta & $\mathrm{X}$ & & $\mathrm{X}$ \\
\hline \multicolumn{4}{|l|}{ Hayley } \\
\hline Marama & & $\mathrm{X}$ & $\mathrm{X}$ \\
\hline Peter & & $\mathrm{X}$ & \\
\hline Ruru & $\mathrm{X}$ & $\mathrm{X}$ & $\mathrm{X}$ \\
\hline Matiu & $\mathrm{X}$ & $\mathrm{X}$ & \\
\hline Jack & & $\mathrm{X}$ & \\
\hline Benjamin & $\mathrm{X}$ & $\mathrm{X}$ & \\
\hline Whina & $\mathrm{X}$ & $\mathrm{X}$ & \\
\hline
\end{tabular}


Most of the children referred to rules such as no talking out of turn, no playing around, and no fidgeting. Another rule was to sit 'nicely' (legs folded, and seated on bottoms) and a final rule involved being attentive or on task. There were mixed responses about the consequences for breaching these rules. For example, some children indicated that if a child was breaking the rules, he or she was unlikely to be chosen by the teacher to actively participate.

An exception concerned attentiveness and on-task behaviours; in contrast to other rule-breaches, some children indicated that if a child behaved inattentively, they were more likely than less to be chosen to participate. It is possible that calling on an inattentive child with a sudden question was a method used by teachers to expose the child's inattentiveness. Other repercussions for contravening the 'pay attention' rule included being moved away from friends, being given tidying chores, or getting 'told off'. Several children also referred to having to sit near the teacher:

Maia (Classroom B): Erm, most of the time [the teacher] says that naughty people sit up the front.

Olivia (Classroom B): You have got to act when they- these are very good examples for chatter, 'don't, stop it'- step one. And if you chatter lots more again and gives her headaches then she says, 'step two.' Do you know what step five is? The Thinking Sheet; it's like a piece of paper that makes you think about the bad things that you have done.

Hana (Classroom C): You can't, you can't, um, play around or just walk away if you want to go to the toilet when other people are in there. You can, you might [have to] change your card colour ${ }^{8}$.

${ }^{8}$ Classroom $\mathrm{C}$ ran a system whereby infractions of the rules might result in a child being required to change the green card, that was displayed next to their name, to orange or red. The children viewed this practice with a high degree of seriousness. 
Mia (Classroom B): Sometimes she [the teacher] kind of asks random people if they are not listening and talking.

Nonetheless, the children also referred to teachers taking a lenient approach for some of the infractions of the rules. For instance, Amelia (Classroom B) described another child who broke the rules when he was upset. The teacher moved him near her, so that she could comfort him. Another example was given by Holly (Classroom B), who explained that drink bottles were not allowed on the mat; however, the teacher let her keep her drink bottle on the mat one day when it was very hot. A further example was Quentin's (Classroom B) explanation that he often got into trouble on the mat; however, once he began to meet the teacher's expectations about behaviour, he was positively reinforced:

Quentin (Classroom B): Once I was a bit naughty and [the teacher] said I had to tidy up the class room afterwards but I was sitting really neatly by my feet and it kind of got me off the hook and I got to go to lunch early instead.

Many children referred to various processes at mat time. One process that the children commonly referred to was that the teacher made demonstrations and delivered explicit instruction or asked questions. Teachers then explained how to do activities. Other pedagogical processes were seen in the following comments:

Paige (Classroom A): If we don't know what something looks like, she puts it up before we, like, do something. And you know, when you're, like, describing something and you need a description? She uses that as, like, her brainstorm. 'Cause she uses that, 'cause she puts up a picture of an animal or something like that, and she, like, points and arrows, and she writes down what we've said. 
Olivia (Classroom B): I put my hand up and I say some silly thing, well not silly, but what I think is the procedure, and then a few people say their answers and the teacher says hands down, and then actually reveals the key to the secret doorway and reveals what procedure is.

Furthermore, many children also indicated that the teachers frequently explained a learning task. For instance:

Trevor (Classroom B): The teacher explains what we have to do and if we have a work sheet and she talks about the worksheet and then when it is time to go off we then go off to our activities that we are doing.

The assisting role of teachers was identified when several of the children referred to the teacher helping them if they floundered when actively participating. For instance, Zara (Classroom B) said that the teacher helped people find the answer if they got stuck. Other typical comments included:

Jasmine (Classroom A): Okay, sometimes... this is [the teacher, points to model], and she's explaining something and someone doesn't understand it, like Paige, and then she sees Paige, and Paige says, 'I don't understand what (laughs) ten divided by three is' and then [the teacher] says, 'Oh, I'll teach you that.

Areta (Classroom C): Normally [the teacher] is like, will talk to us, and we'll tell her what we said, and she helps us.

Nonetheless, fewer children referred to teachers assisting children compared with rules and processes at mat time. In fact, children seemed just as likely to refer to peers helping each other. 


\subsubsection{Children's feelings about mat time}

Children were asked about their overall enjoyment of mat time, and to explain their answer. The majority of children indicated that they either had neutral feelings towards it (52\%), or that they liked or enjoyed mat time (40\%). One child (Jesse, Classroom A) indicated a dislike of mat time. The remaining child's answer was ambiguous (Hayley, Classroom C).

The children shared several factors that they specifically liked or disliked about mat time, which are depicted in Table 5.3. When the children said that they liked mat time, they frequently attached caveats, for example:

Ella (Classroom A): Okay. I actually choose the smiley face because I do feel happy on the mat sometimes.

Interviewer: You do feel happy on the mat sometimes?

Ella: Yeah. I do. Like sitting in the front. That's happy.

There were several commonly occurring caveats evident in the children's responses that influenced their enjoyment of mat time. Sitting next to a friend was the most commonly cited factor attached to children's enjoyment of mat time, with $47 \%$ of the children referring to it. Experiencing opportunities for fostering togetherness was also important for several children, as it gave them opportunity to enjoy the fellowship of the class (24\%). Other important caveats included getting a good seating position (40\%), enjoyable activity (40\%), learning (18\%), and getting rewards for participation (6\%). 
Table 5.3 Coding for Children's Reports on Aspects of Mat Time that are Enjoyable

\begin{tabular}{|c|c|c|c|c|c|c|}
\hline Classroom A & Activity & Learning & Togetherness & Friendship & Rewards & Position \\
\hline Ella & & & & & & $\mathrm{X}$ \\
\hline Ava & $\mathrm{X}$ & & & & & \\
\hline Paige & $\mathrm{X}$ & $\mathrm{X}$ & $\mathrm{X}$ & $\mathrm{X}$ & & $\mathrm{X}$ \\
\hline Nic & & & & $\mathrm{X}$ & & \\
\hline Jasmine & & & & & & \\
\hline Jesse & $\mathrm{X}$ & & & & $\mathrm{X}$ & \\
\hline Alex & $\mathrm{X}$ & & $\mathrm{X}$ & & & \\
\hline Cameron & & & & & & \\
\hline Keira & $\mathrm{X}$ & & & & & \\
\hline Classroom B & & & & & & \\
\hline Sienna & & & & $\mathrm{X}$ & & $\mathrm{X}$ \\
\hline Holly & & $\mathrm{X}$ & & $\mathrm{X}$ & & \\
\hline Maia & & & $\mathrm{X}$ & $\mathrm{X}$ & & $\mathrm{X}$ \\
\hline Rua & & & & $\mathrm{X}$ & & \\
\hline Hunter & $\mathrm{X}$ & & & & & \\
\hline Connor & & & & & & $\mathrm{X}$ \\
\hline Cooper & $\mathrm{X}$ & & & $\mathrm{X}$ & & \\
\hline Fetu & & & $\mathrm{X}$ & & & $\mathrm{X}$ \\
\hline Olivia & $\mathrm{X}$ & $\mathrm{X}$ & & $\mathrm{X}$ & & \\
\hline Chloe & & & & $\mathrm{X}$ & $\mathrm{X}$ & \\
\hline Zoe & $\mathrm{X}$ & & & & & \\
\hline Quentin & $\mathrm{X}$ & & & $\mathrm{X}$ & $\mathrm{X}$ & $\mathrm{X}$ \\
\hline Loto & $\mathrm{X}$ & & & $\mathrm{X}$ & $\mathrm{X}$ & \\
\hline Tane & & & & $\mathrm{X}$ & & \\
\hline Trevor & & & & $\mathrm{X}$ & & $\mathrm{X}$ \\
\hline Masina & & & $\mathrm{X}$ & & & $\mathrm{X}$ \\
\hline Zara & & & $\mathrm{X}$ & & & \\
\hline Amelia & & & & $\mathrm{X}$ & & $\mathrm{X}$ \\
\hline Sefa & & & & & & \\
\hline Mia & & & & & & $\mathrm{X}$ \\
\hline Sean & $\mathrm{X}$ & & & & & \\
\hline Luca & & & & & & $\mathrm{X}$ \\
\hline Logan & & & & & & $\mathrm{X}$ \\
\hline Simon & $\mathrm{X}$ & & $\mathrm{X}$ & $\mathrm{X}$ & & \\
\hline Levi & & & & & & \\
\hline Classroom C & & & & & & \\
\hline Arana & & $\mathrm{X}$ & $\mathrm{X}$ & & & $\mathrm{X}$ \\
\hline Isaac & & $\mathrm{X}$ & $\mathrm{X}$ & & & \\
\hline Rawiri & & & & & & \\
\hline Anahera & $\mathrm{X}$ & $\mathrm{X}$ & & $\mathrm{X}$ & & \\
\hline Hana & & $\mathrm{X}$ & $\mathrm{X}$ & $\mathrm{X}$ & & $\mathrm{X}$ \\
\hline Hauku & $\mathrm{X}$ & & & $\mathrm{X}$ & & $\mathrm{X}$ \\
\hline Areta & $\mathrm{X}$ & & $\mathrm{X}$ & $\mathrm{X}$ & & $\mathrm{X}$ \\
\hline Hayley & & & $\mathrm{X}$ & & & \\
\hline Marama & & $\mathrm{X}$ & & $\mathrm{X}$ & & $\mathrm{X}$ \\
\hline Peter & $\mathrm{X}$ & & & & & \\
\hline Ruru & & & & & & \\
\hline Matiu & & & & $\mathrm{X}$ & & \\
\hline Jack & $\mathrm{X}$ & $\mathrm{X}$ & & $\mathrm{X}$ & & $\mathrm{X}$ \\
\hline Benjamin & $\mathrm{X}$ & & & $\mathrm{X}$ & $\mathrm{X}$ & $\mathrm{X}$ \\
\hline Whina & & & & & & $\mathrm{X}$ \\
\hline
\end{tabular}


Being able to sit next to friend was very important to many children and was one of the dominant themes throughout the interviews. Indeed, nearly half of the children made clear, affiliative comments about sitting by their friends at mat time. Typical comments included:

Alex (Classroom A): I, me and Eddie, are very best friends.

Marama (Classroom A): I usually like to sit next to Anahera 'cause she's my best friend.

Amelia (Classroom B): She's really good when you hurt yourself. She is a really good nice friend to be friends with.

Trevor (Classroom B): When it's time to leave the mat, I don't have to go, 'Tane, where are you?'

A smaller number of children referred to being part of the wider group as an enjoyable aspect of mat time. When children talked about togetherness as a factor of their enjoyment, they generally referred to connecting with others in ways that were promoted by the whole class being together. At times, this was very general, for example:

Alex (Classroom A): I like the lots of people sitting around me.

Zara (Classroom B): I don't like being only a couple of people. There has to be a certain amount of people around me so I don't feel that lonely.

Arana (Classroom C): Um, because we, ah, we make each other happy. 
The mat also provided a positive opportunity to connect with other children with whom they might not typically interact. Paige (Classroom A) indicated that mat time had potential to help her find a playmate when her close friend was away. Other children referred to when the teacher organised the mat time so that children were buddied with others to work with, who they would not ordinarily choose or be chosen by. This seemed especially pertinent for Fetu, given that he was someone who was often left over when the children formed small working groups ${ }^{9}$.

Paige (Classroom A): People are away, they feel sick, like Arearea. She's not here and she's my best friend. So when she's not here.

Fetu (Classroom B): I can share ideas with anyone I want, except sometimes you have to share with some people you don't even know, but I'm okay with that because then I get to meet new people.

Activities were a highly cited factor that related to children's enjoyment. The most popular activities that children referred to specifically included reading books or being read to, and playing games such as Top Cat $^{10}$. In fact, nearly all of the children who cited an activity as an enjoyable aspect of mat time nominated either books or games. Few other activities were nominated; however, a very small number referred to small group and pair discussion, or science experiments. One child said that he enjoyed mat time when the teacher let the children massage each other. In contrast to activities, a small group of children cited learning as a factor of their enjoyment. Such children referred to either the processes of learning, or understanding specific information:

${ }^{9}$ Confirmed by the video observations and his teacher's interview.

10 Top Cat is a mathematics game where equations are issued to an individual child (the defender) and a peer (the challenger). If the defender is first with a correct response, then a new challenger is appointed. However, if the challenger responds first, then they are appointed as the new defender. It is expected that every child will have a turn to compete as challenger. 
Keira (Classroom A): When we do maths, we find out what to do.

Holly (Classroom B): [others] might know stuff I don't know and they tell [the teacher] and then I can learn from it.

Marama (Classroom C): It makes my brain happy.

Jack (Classroom C):We count up to sixty 'cause we're learning about sixty, 'cause one whole hour is sixty minutes so sometimes we go to thirty because half of sixty is thirty minutes on the clock.

However, there were several things about mat time that children did not like, which are represented on Table 5.4. The most frequently nominated was disruption by other children (48\%). Conflict with classmates was nominated by $16 \%$ of the children. Physical discomfort was nominated by $16 \%$ and having a poor seating position was referred to by $28 \%$. The teachers' facilitation was referred to by $10 \%$ of the children, citing reasons such as mat time being boring or too long. 
Table 5.4 Coding of Children's Reports on Aspects of Mat Time that are Disliked

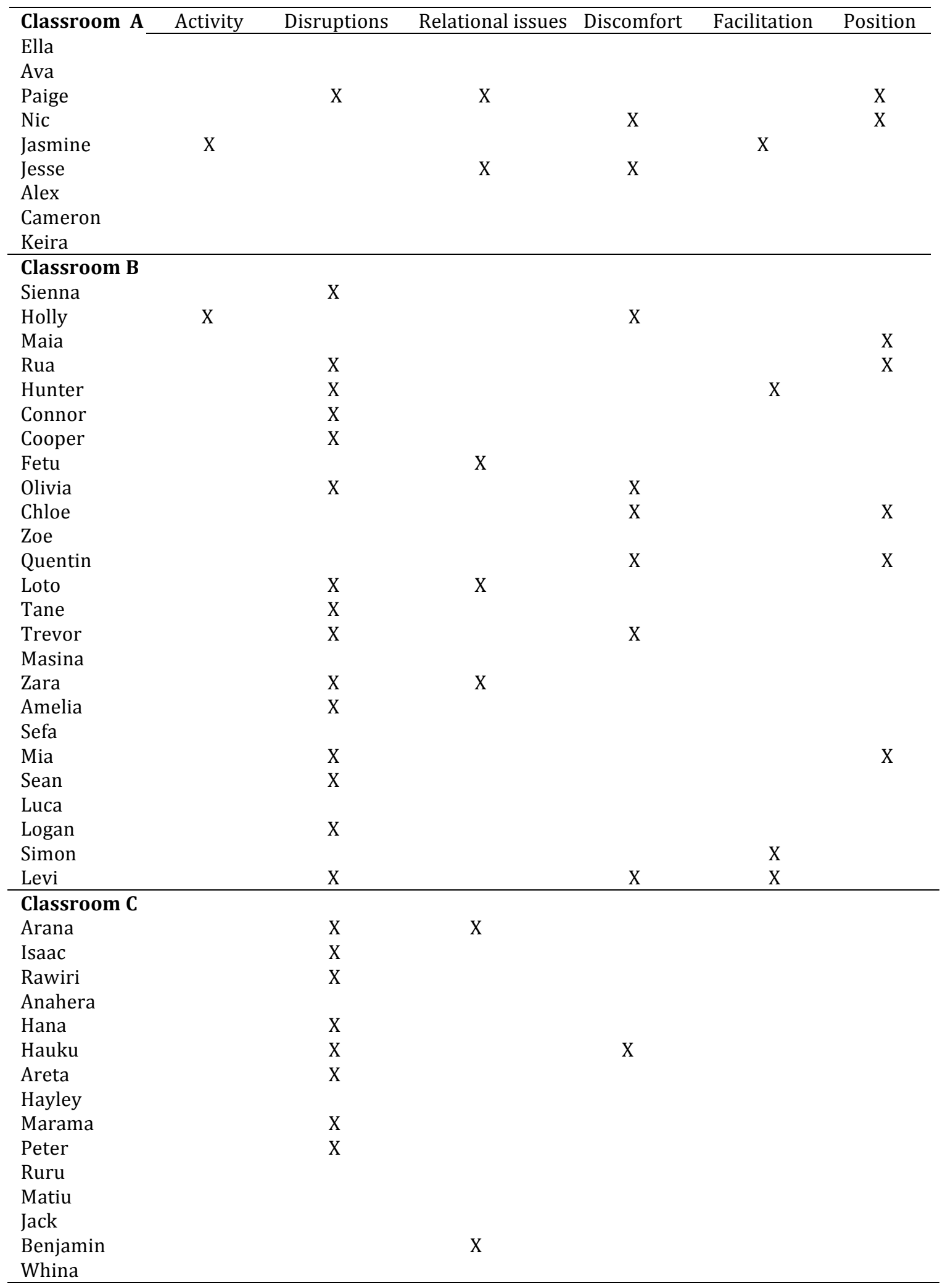


Disruptions were a significant issue for the children and an aspect of the mat that many of them disliked. Several children identified deliberate attempts by their friends to distract them. For example:

Hunter (Classroom B): You know my friend, this guy, he's sometimes behind me and then he starts fiddling with my back and stuff and starts tickling me or something, then I have to move, then he just moves with me.

In contrast, Cooper did not appear to mind being distracted by his friends when he was confident in his understanding of the material that was presented by the teacher. However, when he was less confident about his understanding or knowledge, he preferred to focus on the teacher. He summarised these thoughts as:

Cooper (Classroom B): Everything's complicating, sitting at the front, at the back, at the front. Well, mostly I sit with my friends if I'm confident. I sit with my friends and I sometimes chat. Otherwise, if I'm not confident, I'm moving away, moving away, and if they're being silly I try and not get in trouble.

Aside from the distractions of friends, the most commonly referred to disruptions were other children 'playing around' and other children calling out of turn.

Relational factors caused distractions and negatively impacted certain children's enjoyment of mat time. For some children, this included conflict within their friendship group. This seemed particularly pertinent when they were involved in triadic friendship groupings and the teacher called for pair activities. For example, Olivia (Classroom B) referred to an incident in which the teacher asked the children to find a partner for a discussion on the mat. As per the teacher's instructions, Olivia went with one of her two friends, in this case, Loto. However, 
this meant that her other friend, Maia, had to find a different partner. She indicated that the interaction resulted in acrimony, by stating:

Olivia (Classroom B): I buddied up with Loto, and then Maia had to go with Levi... Enemies! Funny kind of enemies.

Conflict with non-friends was also evident. The conflict often had its beginnings in physical interactions; for example, Areta (Classroom C) reported that conflict arose when other children were touching her hair. One of her friends, Anahera, stated that the teacher had needed to make a rule that children were not allowed to touch each other on the mat in order to avoid fighting. Areta stated:

Areta (Classroom C): Playing with my hair-tie and my hair. It makes me hurt. It makes me go all angry.

A few children indicated that the relational or social aggression of peers negatively impacted their enjoyment of mat time. The aggression appeared to most frequently occur in the playground, but sometimes during class time as well. Fetu (Classroom B) best illustrated this when he explained that he would even give up a coveted front position on the mat in order to avoid a peer who had teased him:

Fetu (Classroom B): Well, sometimes I make unusual choices, like when I sit up the front I go all the way to the back because I don't think it's very good if I'm sitting next to someone I don't like. Interviewer: So, if you were sitting next to someone you didn't like, you would move?

Fetu: Yes. Someone who is mean to me.

Similarly, Jesse (Classroom A) indicated that she could not sit next to Ella, citing reasons associated with name-calling: 
Jesse (Classroom A): Because she called me stupid one day.

Later on in the interview she said that Ella used to be her friend, but now she was "mean". However, in her interview, Ella disclosed that she experienced peer issues also. She said that she did not like mat time because people wanted to buddy up with Jasmine, who was more popular. This had a negative impact on her feelings within the group:

Ella: (Classroom A): It makes me feel like I'm not that important.

Aside from relational factors, some children referred to physical discomfort as something they disliked at mat time. Sometimes this related to feeling squashed and sometimes it referred to physical stiffness or soreness, for example:

Jesse (Classroom A): At the back there's more space to move.

Quentin (Classroom B): Everybody can be squished up the front sometimes.

Sienna (Classroom B): I like sitting at the back because then your neck doesn't get sore, and then me and my friends, my friends came and they're at the front, so I'm sitting at the front.

Interviewer: Why did your neck get sore?

Sienna: Because you need to look up and it starts hurting.

Chloe (Classroom B): You are sitting right in front of the cupboard like this and then your face is shoving onto the cupboard.

The problematic nature of space on the mat was evident in Nic's (Classroom A) account of moving in the crowded space: 
Nic (Classroom A): Um. Some um, sometimes people can get injuries on the mat.

Interviewer: Oh. Do they?

Nic: Yeah. Um. 'Cause somebody might have stepped, if they've just come, stepped on, and if someone is already sitting down, somebody might have stepped on their hand.

Another aspect of mat time that impacted on certain children's enjoyment was seating position in relation to whether they were chosen by the teacher to actively participate. Two children indicated that the teacher rarely chose children to participate if they sat at the back corners:

Maia (Classroom B): Because, sometimes you miss out if you wanted to share the answer, like, you don't usually get to if you're at the back.

Similarly, Rua (Classroom B) suggested that being chosen related to seating position. He asked for a piece of paper and a pen, and drew a map, that depicted positions in relation to being chosen by the teacher. This is reproduced as Figure 5.3. 


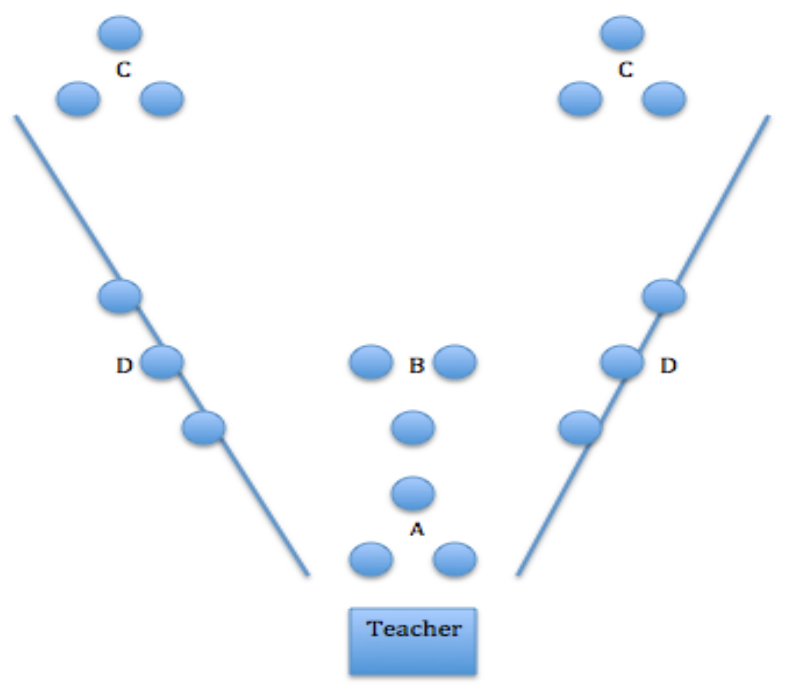

Figure 5.3. Rua's map of seating positions on the mat in relation to the likelihood of being chosen by the teacher for active participation

Rua (Classroom B): If you are around here [indicates three places in front, denoted by 'A' on the map] the teacher will normally pick you and if you are here [directly behind, see 'B'] she might pick you. So ifyou are in the arc shape she will pick you, but if you are here, here or even over here [indicates back positions, see 'C'] ah, in the corners and chatting, chatting, chatting she won't pick you. So, um, imagine this was it. At the back, well, sometimes at the back she might not pick you. If you go here or here or here [indicates the places in front of the teacher, see 'A']. Some people either sit over here and over here [indicates sides, see ' $D$ '], they normally might put their hand up but if you're making a bit of noise and talking to your friends then the teacher is not very likely to pick you. And if you don't want to get distracted, you can actually, there's sort of a triangle that you can sit in, so if I sat here, here or over here [front, see 'A'], those are some of the best places because she can see you better. But if you're right over here [points to back ' $C$ '] or over here [points to sides, see 'D'], even if you're over here, over here [points to middle, see ' $B$ ', shakes head]. 
Very few children cited the teachers' facilitation as something they disliked about mat time. However, Chloe (Classroom B) and Ruru (Classroom C) referred to it as 'boring'. Jasmine (Classroom A) and Hunter (Classroom B) found it too long, and Connor (Classroom B) suggested that the teacher was not clear at times, and said that he did not like it when he did not understand what the teacher said:

Jasmine (Classroom A): I get a bit annoyed because it's a bit annoying because the teacher goes blah blah blah blah blah blah blah when I could go, here. Here's your task...Maybe you should know that it's very long.

Hunter (Classroom B): You know what I think I kinda find annoying about mat time? It's that it gets, like, too like, I stay for hours, and it gets really, really long, and like, well, that's my whole problem with school really. It's too long.

Connor (Classroom B): When she [the teacher] asks us to talk about a really hard thing, sometimes I don't really get what she's meaning sometimes.

\subsubsection{Importance of participation}

Children were asked whether their personal participation was important to them. Children's responses focused on their participation in discussion, giving answers to questions that the teachers asked, or participating in games. This was irrespective of whether they sat in a circle or en bloc. Table 5.5 shows that $70 \%$ said that participation was important, $22 \%$ said that it was not, and $4 \%$ were unsure. One child did not answer the question. 
Table 5.5 Coding of Children's Ratings of the Importance of Participation

\begin{tabular}{|c|c|c|c|}
\hline Classroom A & Important & Not important & Unsure \\
\hline Ella & $\mathrm{X}$ & & \\
\hline Ava & $\mathrm{X}$ & & \\
\hline Paige & $\mathrm{X}$ & & \\
\hline $\mathrm{Nic}$ & & & $\mathrm{X}$ \\
\hline Jasmine & $\mathrm{X}$ & & \\
\hline Jesse & & & $\mathrm{X}$ \\
\hline Alex & $\mathrm{X}$ & & \\
\hline Cameron & $\mathrm{X}$ & & \\
\hline Keira & & & $\mathrm{X}$ \\
\hline \multicolumn{4}{|l|}{ Classroom B } \\
\hline Sienna & & $\mathrm{X}$ & \\
\hline Holly & $\mathrm{X}$ & & \\
\hline Maia & $\mathrm{X}$ & & \\
\hline Rua & $\mathrm{X}$ & & \\
\hline Hunter & $\mathrm{X}$ & & \\
\hline Connor & $\mathrm{X}$ & & \\
\hline Cooper & $\mathrm{X}$ & & \\
\hline Fetu & $\mathrm{X}$ & & \\
\hline Olivia & $\mathrm{X}$ & & \\
\hline Chloe & & $\mathrm{X}$ & \\
\hline Zoe & & $\mathrm{X}$ & \\
\hline Quentin & $\mathrm{X}$ & & \\
\hline Loto & $\mathrm{X}$ & & \\
\hline Tane & & $\mathrm{X}$ & \\
\hline Trevor & $\mathrm{X}$ & & \\
\hline Masina & & $\mathrm{X}$ & \\
\hline Zara & & $\mathrm{X}$ & \\
\hline Amelia & $\mathrm{X}$ & & \\
\hline Sefa & & $\mathrm{X}$ & \\
\hline Mia & $\mathrm{X}$ & & \\
\hline Sean & $\mathrm{X}$ & & \\
\hline Luca & $\mathrm{X}$ & & \\
\hline Logan & $\mathrm{X}$ & & \\
\hline Simon & $\mathrm{X}$ & & \\
\hline Levi & & $\mathrm{X}$ & \\
\hline \multicolumn{4}{|l|}{ Classroom C } \\
\hline Arana & $\mathrm{X}$ & & \\
\hline Isaac & $\mathrm{X}$ & & \\
\hline Rawiri & $\mathrm{X}$ & & \\
\hline Anahera & $\mathrm{X}$ & & \\
\hline Hana & $\mathrm{X}$ & & \\
\hline Hauku & & $\mathrm{X}$ & \\
\hline Areta & $\mathrm{X}$ & & \\
\hline Hayley & & & $\mathrm{X}$ \\
\hline Marama & $\mathrm{X}$ & & \\
\hline Peter & $\mathrm{X}$ & & \\
\hline Ruru & & $\mathrm{X}$ & \\
\hline Matiu & $\mathrm{X}$ & & \\
\hline Jack & $\mathrm{X}$ & & \\
\hline Benjamin & $\mathrm{X}$ & & \\
\hline Whina & $\mathrm{X}$ & & \\
\hline
\end{tabular}


Participation was a very strong theme throughout the interviews. Several subthemes were identified that related to involvement, learning, reputation, behavioural expectations, and reward. Table 5.6 shows the coding for these categories. Participating to be involved was the strongest sub-theme, with typical comments including:

Hunter (Classroom B): Because I really want to share my idea and be really more involved in the class, because I don't really get to answer questions very much.

Olivia (Classroom B): This person has a twin hand, it's going to bust, that's usually me I start to burst.

Interviewer: So, when you've got your hand up, you're dying to be chosen to speak?

Olivia: Yes, because I have always got ideas popping up in my head.

Another sub-theme was that children wanted to participate in order to benefit their own learning or the learning of others. A typical response was made by Marama and Arana (both from Classroom C):

Marama (Classroom C): If we don't share, then we don't learn anything, because, your brain thinks a lot and your questions come out when you're handling it.

Arana (Classroom C): Umm. We learn more stuff. And she teaches us more stuff if we don't know it. Interviewer: So, if you said something that wasn't quite right [the teacher] would-

Arana: -so, if you know, um, like, um, understanding, but like pretending, you don't know, um. Yeah. I'll put my hand up and I'll say, what does understanding mean? And the teacher will tell us. 
Table 5.6 Coding of Children's Reasons for the Importance of Participating

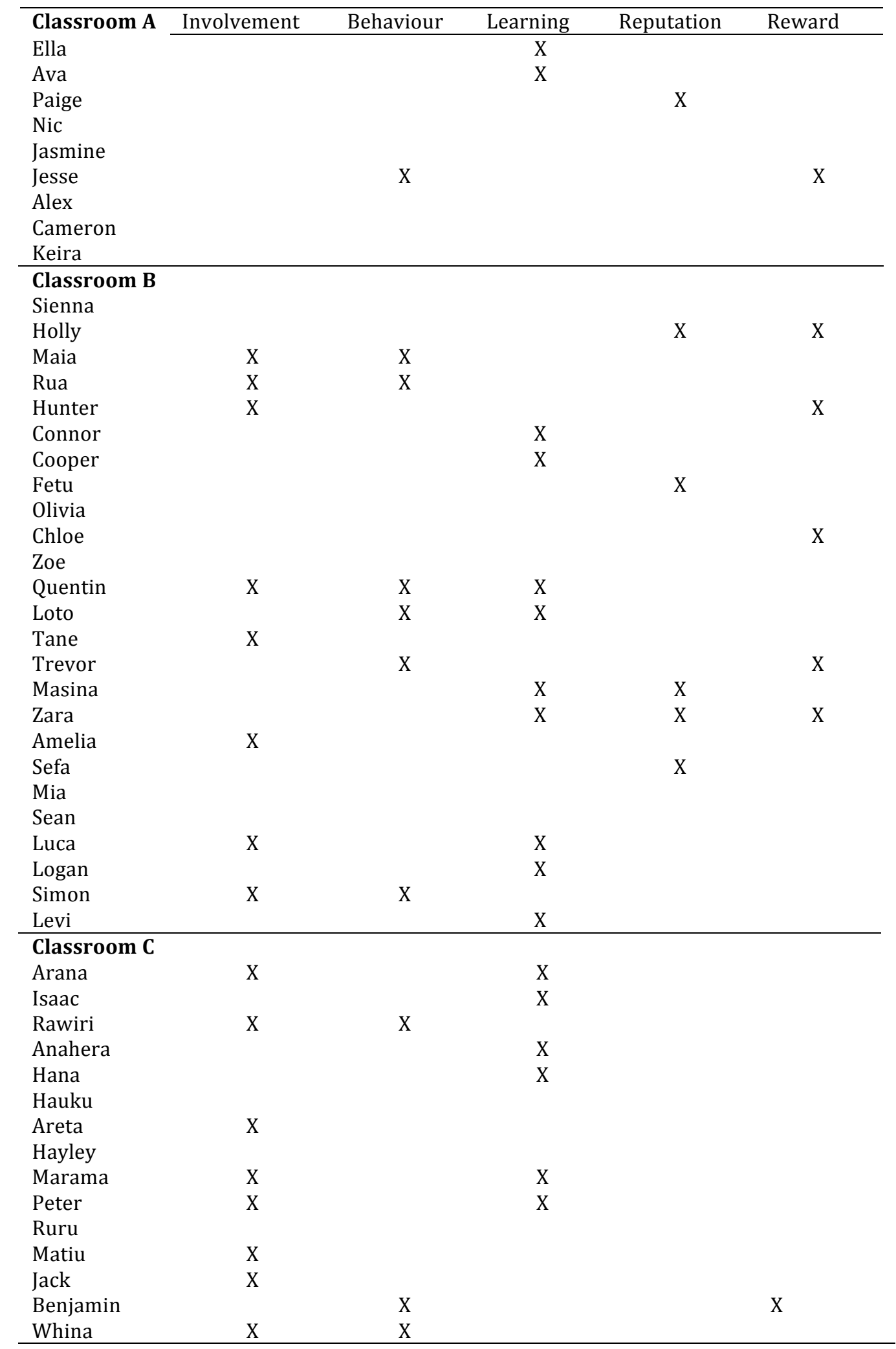


The above reasons appeared to be based on intrinsic motivation; however, one quarter of the children also gave detailed answers that their participation was associated with other people's perceptions of their behaviour; hence this subtheme was called behavioural expectations. For instance, some children said that participation was important in order to comply with the teacher's expectations about behaviour on the mat, such as Maia's comment:

Maia (Classroom B): Because sometimes teachers think you might not really be listening if you don't put your hand up for anything.

Three children indicated that meeting the expectations was possibly formally recognised and rewarded. Jesse (Classroom A) listed several rewards for good behaviour on the mat, including being allowed to go to morning tea first, being chosen as the teacher's special helper, and being allowed to feed the goldfish. Benjamin (Classroom C) explained that he had received a certificate for doing well at mat time. Trevor (Classroom B) said that he had worked hard and got to read his story at mat time. In the case of Chloe (Classroom B), it was not clear whether an actual reward was given or whether she merely desired one:

Chloe (Classroom B): Because I want to get a prize.

Interviewer: So you get a reward if you get the answer right?

Chloe: Yes, I think so. But not really. But I want to get a prize.

A sub-theme emerged that linked children's participation with reputation or peer judgment, in that skillful participation might alert others to how clever they were. For example, Hunter (Classroom B) referred to a peer that he admired, saying, "he is much kind of smarter" and Amelia (Classroom B) described Olivia as someone who always behaves and who is "very good at origami and lots of things". Other relevant comments included: 
Simon (Classroom B): If I get the answer right, then people would think that I'm pretty good at maths.

Quentin (Classroom B): Well it [participation] should be important. Yeah, it's important for lots of people, because some people could be smart, and some people that aren't very smart, they sometimes still share their opinions, and they are actually quite correct.

Furthermore, some of the children suggested that specific peers might make desirable partners or have greater status at mat time based on their judgment of the peers' academic or on-task behaviours. It was evident that certain children were discerning about which peers they would team up with when the teacher called for small group discussions. Benjamin (Classroom C) referred to being selective about his partner during 'pair share' (where children are asked to discuss a topic in pairs):

Benjamin (Classroom C): When we do pair share, it matters who talks to me ...because we share our ideas.

Some judgments about specific children's participation and performance were particularly unfavourable; it was evident that such children had poor academic or behavioural reputations within the peer group. In fact, specific children appeared to use the performance of unpopular peers at mat time as justification to denigrate them. For example, typical comments about specific peers included:

Olivia (Classroom B): These three girls are not so popular, but they are popular for getting into trouble.

Benjamin (Classroom C): He doesn't even think. 
Isaac (Classroom C): [these] people don't put their hands up so they let the smart people do the work for them. They don't put their hand up because they don't wanna talk to anybody 'cause they're lazy.

An outlying theme about participation was related to competition. Several children were highly focused on their own participation in relation to the performance of peers. For example, they referred to not wanting to share ideas because retaining them preserved what they perceived to be an academic advantage over peers. For example:

Loto (Classroom B): I already know some good things that I don't really want to share because they're actually quite special.

He went on to describe that he saved his good ideas for his own work. It was inferred that the excellence of his work earned him kudos with the teacher and his classmates. Levi (Classroom B) shared a similar belief, stating:

\section{Levi (Classroom B): Some of the people might not know anything} about it and they just might steal my idea, say like if you have got a test and it's about that.

Relatively fewer children said that participation was not important, and none from Classroom A. Nonetheless, Table 5.7 shows that of the ten in Classrooms B and $\mathrm{C}$, who indicated that participation was not important, the most common theme was non-specific; in other words, a generalised dislike of participation. Other children were able to give more specific responses, such as personal shyness. 
Table 5.7 Coding of Children's Reasons for Participation Being Not Important

\begin{tabular}{llcccc}
\hline & & $\begin{array}{c}\text { General } \\
\text { dislike }\end{array}$ & $\begin{array}{c}\text { Performance } \\
\text { anxiety }\end{array}$ & Shyness & $\begin{array}{c}\text { Retain } \\
\text { competitive } \\
\text { edge }\end{array}$ \\
\hline Classroom B & $\begin{array}{l}\text { Sienna } \\
\text { Chloe }\end{array}$ & X & & $\mathrm{X}$ & \\
& $\begin{array}{l}\text { Zoe } \\
\text { Tane }\end{array}$ & $\mathrm{X}$ & & $\mathrm{X}$ & \\
& Masina & & $\mathrm{X}$ & & \\
& Zara & $\mathrm{X}$ & $\mathrm{X}$ & $\mathrm{X}$ \\
& Sefa & $\mathrm{X}$ & & \\
Levi & & & & \\
\hline
\end{tabular}

Another reason for participation not being important pertained to children's anxiety about their own performance. Indeed, there were at least two respondents who explicitly worried about giving incorrect answers to their teachers' questions.

Sienna (Classroom B): I'm a bit too shy to talk in the whole group.

Mia (classroom B): Because when I get something wrong everyone kind of looks at me and I don't really like it. The main thing is that I think people might tell everyone, but I don't think that normally happens.

It is important to note that across the 49 children, many gave a strong impression that the three teachers promoted the value of children's attempts more highly than their performance at mat time. A pattern emerged whereby just over half of the children (56\%) indicated that it was more important to try or make an effort, as opposed to being correct on the mat. A typical comment was: 
Jack (Classroom C): Nah, it's not really important if I get it wrong or right, 'cause at least I share my ideas, and 'cause that we don't, we done, we did the cross country, we don't matter if we come first or third, or in the middle. We don't worry about third place, it's just about coming.

Nevertheless, despite the fact that many children said this, the validity of the statement was brought into question given that some of the children who said that being correct was not important also clearly demonstrated pleasure about getting responses correct. It could be that mastery over performance was something that the teachers emphasised, and which the children repeated. In fact, Whina (Classroom C), described the teacher's reaction to Arana celebrating his correct response:

Whina (Classroom C): When Arana got it right, he says, 'yay' and [the teacher] says, 'aw' (Whina makes a simultaneous accompanying expression whereby she rolls her eyes in an exasperated way).

A final sub-theme evident about participation, was named helping friends. It was very clear that children's access to friendships was important to their participation and academic performance at mat time. For example, the following statements illustrated the influence of a friend:

Benjamin (Classroom C):[I sit by my friend] 'cause sometimes he tells me some ideas when I don't put my hand up and there's some stuff so I can share ideas. He says 'Benjamin' and he shows me his work. He tells me what, what to put up my hand and he tells me what ideas I should say. 
Zoe (Classroom B): Sometimes when we're going off, some people can't know what we're doing 'cause they didn't really understand, so their friends might tell them.

In contrast, it was clear that a very small number of children were unwilling to help non-friends. One example was seen in Paige's (Classroom A) interview, when she complained that Ella tried to copy her on the mat one day:

Paige (Classroom A): Well, sometimes it's like Ella and some other people is copying and then [the teacher] says you can move your desk. That's what's happened to me 'cause she had to go to the red table and she had to sit there while I was doing my things and it was a bit hard.

Finally, some children indicated that working with their friends was easier than working with non-friends at mat time. This is evinced by the following excerpts:

Mia (Classroom B): Well, there's my friend, and usually we have a little chat and when we're buddies we don't have to work to understand.

Marama (Classroom C): I usually like to sit next to Anahera 'cause she's my best friend.

Interviewer: Do you think it's important who you sit with? Is it important to you?

Marama: Yeah...she shares, and she thinks about ideas too...and we share our ideas.

While the majority of children referred to helping friends to participate effectively, or being helped by friends, two children asserted that they liked to help non-friends. Areta (Classroom C) indicated that she invited non-friends, who 
had been excluded from other groups, to join her and her friends with small group discussions at mat time and Marama (Classroom C) identified two non-friend peers as requiring assistance with their work. These specific children were the same ones that had attracted negative comments from Benjamin and Isaac:

Areta (Classroom C): I say come with me and my friends...some people get some more new friends.

Marama (Classroom C): They both need help and so that's why I like to sit next to them. And help them.

One child spoke about the importance of the teacher assisting her to secure a participatory role. Whina (Classroom C) explained that she felt shy at mat time, yet at the same time, she seemed to want to take an active role. She said that if she sat near the teacher, the teacher most likely would notice that she was not participating and choose her:

Whina (Classroom C): Um, I hide sometimes, then [the teacher] tells me what to say.

Interviewer: You hide?

Whina: I'm shy.

Interviewer: So sitting here [near the teacher], does that make you less shy?

Whina: Yeah.

Interviewer: Okay. So, why does sitting here make you feel less shy?

Whina: 'Cause I, I always sit there and she sees, and I always get picked and get snapped not putting my hand up.

Interviewer: So this is a place that [the teacher] sees you really well?

Whina: Yeah. 


\subsubsection{A closer view of children who rated their participation as unimportant}

Further analysis was undertaken of the 10 children who rather their participation as unimportant in order to see if there was any facets that unified their experience. Understanding such facets might be useful in adapting the interactional milieu in order to encourage those children's active participation. Table 5.8 shows that the children who rated their participation as unimportant had few things in common. When their enjoyment was studied, six of them held neutral feelings about mat time, whereas four of them said that they enjoyed it. Six of these children liked the relational aspects of mat time, such as togetherness and friendship. One child disliked relational issues, and five said that they disliked disruptions. Six could see some benefit in their participation, even though they said that it was not important. Benefits included rewards, being involved, learning, and reputation.

Encouraging the involvement of such children is likely to require complex strategies, which are designed on an individual basis. For instance, Zara (Classroom B) enjoyed the feeling of the whole class being together; however, she was bothered by disruption and relational factors. She was concerned about her own performance and seemed to have a poor view of her own academic confidence, as evinced by the following statement:

Zara (Classroom B): Sometimes I work something out and I say,' no that is not right', and I keep on doing that. And once I think, 'oh, that's right' I put my hand up, and [the teacher] says, 'what' and I say 'I forgot.' It's kind of hard for me because I think the answer might be wrong, so I don't do it.

Nevertheless, she recognised that the teacher could offer assistance and was motivated by the possibility of reward. This contrasted with Tane (Classroom B), who reported that he enjoyed mat time, wanted to be involved but felt too shy to take part. Nevertheless, he valued friendship, so for Tane, the possibility of working with a friend in a small group might enable his participation. 
Table 5.8 Overview of Responses for Children who rated Their Participation as Not Important

\begin{tabular}{|c|c|c|c|c|c|c|}
\hline & Enjoyment & Liked aspects & Disliked aspects & $\begin{array}{l}\text { Reasons against } \\
\text { participation }\end{array}$ & $\begin{array}{l}\text { Reasons for } \\
\text { participation }\end{array}$ & $\begin{array}{l}\text { What happens } \\
\text { at mat time }\end{array}$ \\
\hline \multicolumn{7}{|c|}{ Classroom B } \\
\hline Sienna & Neutral & $\begin{array}{l}\text { Friendship } \\
\text { Seating position }\end{array}$ & Disruptions & Shyness & - & Rules \\
\hline Chloe & Happy & $\begin{array}{l}\text { Friendship and } \\
\text { rewards }\end{array}$ & $\begin{array}{l}\text { Discomfort and } \\
\text { Seating position }\end{array}$ & Dislike & Reward & - \\
\hline Zoe & Neutral & Activity & - & Dislike & - & $\begin{array}{l}\text { Rules and } \\
\text { processes }\end{array}$ \\
\hline Tane & Happy & Friendship & Disruptions & Shyness & Involvement & $\begin{array}{l}\text { Rules and } \\
\text { assistance }\end{array}$ \\
\hline Masina & Happy & $\begin{array}{l}\text { Togetherness } \\
\text { seating position }\end{array}$ & - & Performance & $\begin{array}{l}\text { Learning and } \\
\text { reputation }\end{array}$ & - \\
\hline Zara & Neutral & Togetherness & $\begin{array}{l}\text { Disruptions and } \\
\text { relational issues }\end{array}$ & Performance & Reward & Assistance \\
\hline Sefa & Neutral & - & - & $\begin{array}{l}\text { Dislike and } \\
\text { performance }\end{array}$ & Reputation & $\begin{array}{l}\text { Rules and } \\
\text { processes }\end{array}$ \\
\hline Levi & Neutral & - & $\begin{array}{l}\text { Disruptions } \\
\text { Discomfort } \\
\text { Facilitation }\end{array}$ & Competitiveness & Learning & Processes \\
\hline \multicolumn{7}{|c|}{ Classroom C } \\
\hline Hauku & Enjoy & $\begin{array}{l}\text { Activity } \\
\text { Friendship } \\
\text { Rewards }\end{array}$ & Disruptions & Dislike & - & Rules \\
\hline Ruru & Neutral & - & - & Dislike & - & $\begin{array}{l}\text { Rules, processes } \\
\text { and assistance }\end{array}$ \\
\hline
\end{tabular}




\subsubsection{A closer view of confident children}

A small group of 7 children were similar in their reports about feeling confident at mat time. This was particularly true for their performance in competitive games. It seemed that these children might be those who experienced goodness-of-fit between their own dispositions and knowledge, and the mat time milieu. As Table 5.9 indicates, not all of the children in this group reported that they enjoyed mat time; however, each of them placed high value on the importance of their personal participation. In addition, they each appeared to possess a high degree of confidence in their own academic abilities. In the following example, Quentin spoke enthusiastically about a competitive game, Top Cat, and expressed a self-appraisal on his own performance:

Quentin (Classroom B): Have you seen us play top cat because I have won quite a bit, I've won about I don't know, seven... three this term, four last term and the term before that about six. People like Rua, is a really smart man. He always gets to the final but sometimes me, and Connor and Loto, we can knock him out. But sometimes he knocks us out. And I am good at the hard questions, so when it comes to the easy questions, it comes to a battle of muddle.

Most of the children in this group spoke about both individualised competition and competing in relation to their friends with equal importance. This was evident in Quentin's statement above. Another clear example was given by Amelia (Classroom B) who initially discussed a group task that rewarded the winners with stickers, then later in her interview, referenced the more individualised competition required when playing Top Cat. 
Table 5.9. Overview of Responses of Children who indicated that they are Confident at Mat Time

\begin{tabular}{|c|c|c|c|c|c|}
\hline Class B & Enjoyment & $\begin{array}{l}\text { Judges } \\
\text { peers }\end{array}$ & $\begin{array}{l}\text { Participation as } \\
\text { important }\end{array}$ & $\begin{array}{c}\text { Reason for } \\
\text { participation }\end{array}$ & $\begin{array}{l}\text { Liked aspects } \\
\text { of mat time }\end{array}$ \\
\hline Cooper & $\mathrm{X}$ & $\mathrm{X}$ & $\mathrm{X}$ & Learning & $\begin{array}{c}\text { Activity } \\
\text { Friendship }\end{array}$ \\
\hline Quentin & & X & X & $\begin{array}{c}\text { Involvement } \\
\text { Behaviour } \\
\text { Learning }\end{array}$ & $\begin{array}{l}\text { Activity } \\
\text { Friendship } \\
\text { Rewards } \\
\text { Position }\end{array}$ \\
\hline Loto & & $\mathrm{X}$ & X & $\begin{array}{l}\text { Behaviour } \\
\text { Learning }\end{array}$ & $\begin{array}{l}\text { Activity } \\
\text { Friendship } \\
\text { Rewards }\end{array}$ \\
\hline Amelia & & $\mathrm{X}$ & $\mathrm{X}$ & Involvement & $\begin{array}{l}\text { Friendship } \\
\text { Position }\end{array}$ \\
\hline $\begin{array}{l}\text { Class C } \\
\text { Isaac }\end{array}$ & & X & X & Learning & $\begin{array}{c}\text { Learning } \\
\text { Togetherness }\end{array}$ \\
\hline Benjamin & $\mathrm{X}$ & X & $\mathrm{X}$ & $\begin{array}{l}\text { Behaviour } \\
\text { Rewards }\end{array}$ & $\begin{array}{l}\text { Activity } \\
\text { Friendship } \\
\text { Rewards } \\
\text { Position }\end{array}$ \\
\hline Jack & X & $\mathrm{X}$ & $\mathrm{X}$ & Involvement & $\begin{array}{l}\text { Activity } \\
\text { Learning } \\
\text { Friendship } \\
\text { Position }\end{array}$ \\
\hline
\end{tabular}

Although these children judged their own performance at mat time, they did not seem overly concerned about their academic reputations within the wider group. Nevertheless, each of them made judgments about the academic performances of their peers. Such judgments ranged from criticism to simple observations. Irrespective, they potentially demonstrated a close observation of their peers' responses to tasks or questions at mat time.

Another commonality between the children in this small group was that they articulated sophisticated strategies for achieving their goals, far more so than children who rated their performance as unimportant. For instance, playing around with his friends at mat time was important to Quentin (Classroom B). 
Even though he stated the front was a desirable seating position, he often chose to sit at the back because he believed he was less noticeable to the teacher:

Quentin (Classroom B):Well, it could sometimes be the worst place if you are somebody like me or my friends because if the teacher sees you, if you are kind of at the back, they don't always see you if you're, kind of, like, behind people."

Other children's goals were met with the same degree of strategy. For instance, Benjamin (Classroom C) factored in the teacher's practice of 'going around the circle' to hear the children's ideas. He found that if he sat somewhere in the middle, he could have more time to formulate a good response:

Benjamin (Classroom C): She'd have to get, um, six people over to me...I want to sit there so I get time to think when she asks the questions.

Similarly, Jack (Classroom C) was strategic in his goal to be chosen for an active role. He believed that the teacher looked most frequently at Arana; therefore, his strategy to attract the teacher's attention and be chosen involved sitting next to Arana and catching the teacher's eye. He stated:

Jack (Classroom C): 'Cause [the teacher] usually looks to Anahera ...well I usually sit next to Arana 'cause [the teacher] can see me. [The teacher] usually looks that way and I put up my thumb and she sees me. She looks at people who normally work, like Anahera and Arana. So I sit by Arana so I can actually share my ideas.

Such a strategy implies a degree of social confidence in being able to choose whom to sit next to. This was another commonality that the children in this group had; 
they each had access to a specific friendship group within the classroom, which most likely generated feelings of possessing some degree of social support.

\subsection{Summary of findings}

Seating position was a particularly important factor for many of the children. The findings unambiguously indicated that in classrooms $\mathrm{A}$ and $\mathrm{B}$, which used en bloc configurations, the front centre was the most desired position. However, the children who sat in the circle, in Classroom $C$, had more distributed seating preferences. Irrespective of children's preferences for specific seating positions, their criteria for considering where to sit were similar. The criteria that the children in all of the classrooms used included proximity to their friends, and whether a position would enable better focus or teacher-attention. Many children in Classroom B (with its en bloc configuration) wanted to sit where they could hear well. Hearing was not an issue raised by any of the children from classroom C, which used a circular configuration, or Classroom A, which had a smaller group size.

When children were asked about what happens on the mat, their answers were mostly associated with teacher-directed activity. There was a strong tendency to discuss certain rules and their teacher's role in maintaining these. Some rules that were commonly referred to included sitting 'nicely' (back straight and legs crossed), or being attentive, not calling out, and not playing around. For many children their peers' disruptive behaviour was concerning and they described their teacher's efforts to curb distracting behaviours, such as seating specific children near the front, or directing quick-fire questions at children behaving disruptively. In addition, over half of the children talked about teachers' pedagogical processes such as explaining a task, delivering instruction, or asking questions. A smaller number of children referred to the teacher assisting their learning. Most of the responses were in relation to discussion or instruction. 
It was clear that many children's enjoyment of mat time was dependent upon certain caveats. For half of the children, mat time was enjoyable when they were able to sit with a friend or work with a friend. For many children, there were several benefits, such as knowing where a friend was when the mat time concluded. This was especially relevant when a break was scheduled. Another positive factor of mat time was obtaining a desirable seating position. The third most prevalent enjoyable factor was the provision of particular activities that interested children, specifically games and reading books.

Nonetheless, children did not enjoy all aspects of mat time. Distractions caused by other children emerged as the most disliked aspect of mat time, with over half of the children referring to this. The most prevalent distractions were other children playing around, followed by them calling out of turn or fidgeting. Moreover, several children raised concerns about peer issues such as conflict within friendship groups. Nonetheless, conflict also occurred between children and their other classmates, as opposed to friends. The children frequently referred to physical disruptions as a cause of distraction and conflict (for example, someone playing with another child's hair, or being squashed). A few children were distracted by relational or social aggression by peers that had occurred at other times, off the mat. Finally, a small number of children did not enjoy aspects of mat time facilitation, such as its length or not being chosen to actively participate.

Nearly three quarters of the children indicated that actively participating at mat time was important. A variety of reasons were given, such as active participation being important for learning, and that it was generally good to be involved. Several children also discussed aspects of participation that relate to reputation. It is likely that the children in this study were cognisant of the academic reputations of their peers. Indeed, many children referred to specific classmates as being 'smart' or not, based on their performance at mat time. Of interest, several children referred to their own reputations as being 'clever' or 'smart'. In addition, 
children's reputations as academic, or not, might have contributed to their preferences for certain peers in pair share or other small group discussion tasks.

In contrast, a smaller number of 10 children rated participation as unimportant; however, the elaborations of some of these children indicated that this was linked to fear of social humiliation, rather than truly seeing participation as unimportant. The children predominantly gave two key reasons: concern about giving 'correct' responses to the teachers' questions, and feeling intimidated at the prospect of sharing ideas in front of the group. A very small number of children preferred to avoid sharing their ideas or answers on the mat in the belief that this gave them an academic advantage. These children indicated that their answers or ideas were very good, and that they were concerned that others might 'steal' them.

In contrast, seven children expressed high confidence in their own abilities at mat time. Each of these children appeared to be unconcerned about their academic reputations, and appraised the performances of their peers. This may have contributed to their willingness to engage in competitive activities. In addition, they seemed to have good access to particular friendship groups, which may have contributed to their confidence. They rated their personal participation as important and also seemed to possess sophisticated strategies for achieving their goals.

Overall, children's relationships with peers seemed to have an influence on their involvement and enjoyment. For instance, some children felt conflicted when they wanted to focus on the teacher but their friend wanted to chat. Friendship also presented difficulties for some children in triadic friendship groups, when the teacher wanted them to work in pairs, forcing the exclusion of one child. In contrast, several children referred to the important role of their friends in assisting them to secure participatory roles and share good answers or ideas. It appeared that some children felt more confident about actively taking part at mat time when they sat next to a friend, and some children might have found it easier 
to work with a friend than a non-friend. Friendship was so important to some children that they were willing to sit in places that caused them physical discomfort in order to stay next to their friends.

One or two children in each class seemed to have poor reputations or low social status. Only two children indicated that they were willing to sit next to such children, despite others being seemingly aware of their vulnerable standing. Both of these children indicated that their inclusive behaviour was because their peers required assistance or were excluded from other groups in small group work on the mat. They referred to helping their peers with answers or work, and inviting them as work partners.

\subsection{Discussion}

\subsubsection{Children's impressions of mat time}

While many children reported that they found mat time enjoyable, many others held neutral feelings about it. The children's perceptions about their own enjoyment of mat time in the present study were, on average, lower than previous studies have indicated (see Collins, 2013; Hong, 1995; Moss \& Wilson, 1998). Moreover, even when children did indicate that they enjoyed mat time, their enjoyment was often dependent on certain caveats, such as being able to sit near friends or obtain a desirable seating position. Another frequently cited reason for enjoyment concerned likeable activities, namely games and being read to. This is consistent with findings of previous studies (for example, Cefai et al., 2014; Lown, 2002).

The most disliked aspect of mat time was disruptions by peers. Two commonly reported disruptions were others calling out of turn and playing around. Sometimes, the disruptions involved a friend, but more often than not it involved other peers who were not friends. Given that disruptive behaviour can have a negative impact on a learning environment (Cook et al., 2014), it is important to 
understand why some children disrupted the focus of their peers. One possibility, consistent with the findings of the present study, is that some children who are not in a seating position that allows them to easily see or hear what is happening become disengaged or bored, leading to disruptive behaviour. Similarly, some students may become disruptive if they perceive a task to be unchallenging (Baum, Olenchak \& Owen, 1998), if they have averse feelings toward the task (Cook, et al., 2014), or if they are preoccupied with friendship or social factors (Asher \& McDonald, 2010). Reasons might also be developmental, in that particular children might still have been developing skills in effortful control, such as regulating attention, delaying gratification, and controlling impulses (Valiente, Lemery-Chalfant, Swanson, \& Reiser, 2008), or might not yet be emotionally ready for school (Raver, 2004).

Regardless of the reason for disruption, Cook, et al. (2014) and Vargo (2008) suggest that some teachers unwittingly maintain disruptive behaviour through their positive responses to it. Certainly, the children cited sitting near the teacher as being highly desirable, yet several indicated that their teacher required disruptive peers to move to those seating positions within the teacher's close proximity. This might be a positive reinforcement because, according to several children, active participation in certain tasks is desirable and that there is a better chance of securing that participation for those in positions at the front.

\subsubsection{Children's views on participation and engagement at mat time}

In the present study, there was evidence that children who were perceived as struggling academically experienced difficulty in accessing partnerships with peers at mat time without a teacher's intervention or the assistance of the few children who are willing to help them. There was a sense that some children found it difficult to acquire active roles at mat time. If teachers overlook socially neglected or rejected children by not assisting them to participate (Danielewicz et al., 1996), there are real concerns in relation to equity. When rejected children are consistently excluded from active participation it is likely to impact on their 
academic performance (Wentzel \& Asher, 1995). For instance, there are plausible links between active participation, children's engagement, and learning (Hidi \& Renninger, 2006; Wood et al., 2009; Zaghlawan \& Ostrosky, 2011).

Deliberate focus on the teacher's facilitation practices at mat time with regard to the means of assigning partners is important; recent studies have shown that such practices influence children's peer relationships. For example, Asher and McDonald (2010) and Corsaro (1985) indicated that young children often perceive friends as those with whom they engage in activities. It follows that if a child remains 'unchosen' in the classroom, it may impact the child's standing and self-perception as a friend.

For a small number of children, active participation was seen to have an impact on their reputation within a peer group. Children are generally keen observers of the interactions of peers and make judgments about the academic ability of others. These judgments partly influence whether a child is going to be chosen or not for inclusion in group or pair tasks (Droege \& Stipek, 1993). In fact, Gest, Domitrovich, \& Welsh, (2005) observed that:

Peer academic reputations were also associated with changes over time in children's teacher-rated academic effort and skills, suggesting they may be a marker of differential peer experiences that have implications for academic achievement and skill development (p. 342).

This study indicated that the reputational effect of mat time impacted on specific children's willingness to participate. Nonetheless, given that research about children's academic performance has shown correlations with their acceptability to a peer group (Chen, Hughes, Liew, \& Kwok, 2010; Gest, et al., 2005), in the present study only a very small number of children were concerned about giving correct responses. This finding might be attributable to teaching practices; several 
children mentioned that, in their classroom, it was more important to try than to be correct. Notwithstanding, certain children did express anxiety about being incorrect and these children indicated that this anxiety impacted on their participation. Moreover, several children who stated that it was more important to try clearly relished those times when they did issue correct answers. This suggests that at least some of the children might have parroted their teachers' edict on this matter, rather than believing it themselves.

It was evident that the teachers employed methods such as 'pair share' and 'discussion doughnuts' to vary the type of participation required. Both of these practices enabled children to share their ideas with one peer or a small group, rather than the entire class. This may have held greater safety for certain children who were reticent about actively participating in the wider group scenario. However, there are likely to be other solutions that are worthy of consideration. Chen and Looi (2011) noted that the use of computer technology, such as 'Group Scribble', enabled a wider range of children to share ideas through pictures and memos prior to discussion. They found that despite active participation being voluntary, every child participated and active participation was distributed evenly across the group. These findings suggested that interactive technology offers some exciting possibilities to enhance active participation at mat time.

\subsubsection{Children's social worlds at mat time}

Examination of peer culture should encapsulate the concerns, and values that are evident in the actions of those belonging to that peer culture (Corsaro, 2012). In addition, McLaren (1999) suggests that research of the peer under-life in classrooms should consider power dynamics, and whose interests the peerpractices, rituals, and culture serve. However, in the present study, it appeared that there was, not one, but several peer cultures within each class. Moreover, each sub-group probably communicated different routines, concerns. Nonetheless, there were routines, concerns, and values that were common across the peer group of each class. 
A predominant concern was the competition for a desired position at the front of the mat, when the class used an en bloc configuration. In Classrooms A and B, a high degree of competition was evident for the front-centre positions, meaning that the most effective competitors were more likely to sit there. It is possible that teachers might be unaware of the degree of competition for the front, even though the children describe it as being very crowded. For instance, Hong's (1995) description of children's seating positions on the mat suggests that children have a great deal of choice over where to sit. She stated that:

Where to sit is up to the children...[They] show different preferences and sensitivities in selecting a good place to sit and someone with whom they would like to sit. They instinctively know which would be the best for themselves. (p. 220)

However, the present study indicates that while some children might instinctively know that the front position is the best for their learning, not all children are able to sit there. Certain philosophical issues must be explored. If there are academic or social benefits accruing from a front seat, competitive behaviour potentially allows individual children to promote their own interests over those of others (Watters, 2013). This raises the issue of whether teachers should address such competitive behaviour from a moral perspective in order to promote fairness to less competitive peers. For example, teachers might change the configuration of the mat time to reduce competition and inequity. Another suggestion would be for them to actively monitor how even-handed they are in selecting children for active participation, or in delivery of their feedback to children. In fact, failure to address such competitive behaviour is antithetical to the New Zealand Curriculum Framework's key value of 'equity', which posits that children will develop a sense of social justice (Ministry of Education, 2007, p.10). 
It seems that the circle configuration ameliorated at least some of the competition for a preferred seating position that was evident in the free-seating, en bloc mat times. This might explain the popularity of a circle configuration in pedagogies aimed at promoting group cohesiveness, such as 'Magic Circles' and 'Golden Circles' (Mosley, 1998; White, 1999). Furthermore, it seems that circular configurations yield other benefits. For instance, Marx, Fuhrer, and Hartig (2000) found that the children asked more questions in a semi-circle seating format than in a row format.

A second concern of the peer culture evident at mat time was relational; it seemed important for many of the children to have a friend to affiliate with. Indeed, Corsaro $(1985,2012)$ asserts that children's relationships are fundamental to peer cultures. In the present study, friendships may have assisted certain children to mitigate competitive and hierarchical elements of mat time. For instance, there was evidence of friends helping each other by sharing their good ideas, and several children indicated that it was easier to work with their friend than other peers. Each of these are important points to consider, because they allude to the possibility that participatory performance at mat time was important to certain children. As such, performing well may have social benefits, such as an academic reputation or teacher praise, despite many children saying that it did not matter if mistakes were made at mat time.

For several children, their friendships enabled their participation; therefore, it is important to consider the experiences and possible outcomes for children who do not have a close friend, or who were referred to by peers in a negative light. It should be noted that in this study, that these children were described by some of their peers as performing poorly at mat time. Asher and McDonald (2010) assert that children who have healthy friendships are more likely to feel well adjusted to school and be more involved in class. Conversely, when children experience friendship difficulties, such as being consistently rejected by a friend, they can experience decreased self-esteem and increased anxiety. Notwithstanding, Study 
Two also found that there were children who had friends, but who still experienced difficulties that potentially impaired their mat time participation and performance; for instance, where a friend was an undesired distracting influence.

In Classroom C, a small group of children demonstrated different values to their peers in relation to the inclusion of children with low social status. For two children in particular, it seemed important to assist such children. Given that the children with low social status also had poor academic reputations among the peer group, helpful peers risked having their joint performance with a low-status peer negatively judged. It seems likely that these children were motivated by different concerns to those of their peers. Buhrmester (1996), and Corsaro (2011) suggest that children's goals differ in varying circumstances. In the case of mat time, it could be that some children aimed to enhance their own reputations and agency, whereas helpful peers strived to achieve aims that were more benevolent.

A close focus was placed on a group of 10 children who rated their performance as unimportant. These children seemed to have little in common, although it seems unlikely that they authentically believed that their participation was, indeed, unimportant. What might be more likely, is that social or academic anxiety deterred them from participating. As such, teachers would need to think very carefully about the most effective ways to motivate these children to actively take part. Any pedagogical strategies would require an individualised focus; for example, one child might be motivated by rewards, whereas another might be induced to take part if cooperation with a well-known and safe peer is allowed.

A second group of children that stood out from the rest of the peer group in the three classes were those who seemed particularly confident. They had identified themselves as being academically competent and rated their personal participation as important. Moreover, they each indicated that they had membership to specific friendship groups; therefore they most likely possessed 
social support. Finally, these children appeared to have a sophisticated knowledge of the teachers' practices and a range of strategies to achieve their goals.

McClelland (1961) posited that human beings have three basic requirements: affiliation, personal achievement, and power (status and recognition). It seems likely that the behaviour of both the overtly confident, and less confident, children differed depending on which requirement they tried to fulfill. For instance, certain social dynamics, relating to McClelland's taxonomy of needs, were evident with one friendship group of children in Classroom B. They frequently sat at the back of the mat in order to escape the teacher's attention and play around. There was a strong sense of their bonding at such times, which relates to the notion of affiliation. Nevertheless, some members only played around when they felt confident about the material being presented. As such, it seems that they were not willing to risk their social reputations in their bid to play around. This might relate to their feelings of personal achievement or need for status.

Another way to interpret the behaviour of this specific group is that they may have been motivated by several concurrent goals. First, the teacher's official stance is that children are required to be attentive at mat time; and, playing around challenges this official rule. Corsaro $(1985,2012)$ suggests that such challenges allow children to reject adult control and to assert a sense of their own agency. However, children tend to attempt to keep their playing around hidden from the teacher's view. This subterfuge binds them together and contributes to their sense of groupness. This certainly seemed to be the case in Milman's (2009) study of morning meeting, whereby specific children refused to attend to the teacher or the allocated task as a hidden mechanism for challenging the norm or expectations. The example of this single group serves to illustrate the social complexities inherent when it comes to participation within the social milieu of mat time. 


\subsection{Conclusions}

Opportunity to engage in effective and active participation has a reputational element in children's peer cultures. Children appear to be close observers of the performances of their peers at mat time. They form both negative and positive judgments about their peers, which might influence their willingness to work cooperatively with specific peers at other times. As such, participation has hidden competitive elements that relate to children's social structures. Moreover, the competitive and reputational elements of mat time might preclude the desire of certain children to participate, causing them to consider their personal participation as unimportant.

Teachers must reflect on cases in which specific children do not participate or view their participation as unimportant. For instance, specific children's underlying anxieties must be uncovered and sensitively responded to in order to encourage such children to take part. In addition, children, who have a friend in the class, might find it easier to participate, whereas other children without a friend might struggle to find peer support without assistance.

Children indicated that seating position is also crucial to effective participation. Specifically, the front positions in free-seated, en bloc configurations are highly sought after. Reasons for the desirability of the front seat tend towards academic advantages, such as being able to see better, or being better able to attract the teacher's attention. As such, teachers should review other seating configurations, or examine ways to distribute any perceived or real academic advantages to sitting in the front. 


\section{CHAPTER SIX \\ Discussion}

\subsection{Introduction}

Within the early years of primary school, there are several interactional contexts in which children are required to learn as part of the whole class; mat time is one of them. Given that a child is situated within the wider social group on the mat, it is likely that he or she will receive and construct messages about how to behave, how to treat others, and how others perceive them. These factors most likely, in turn, impact on individual children's participation and self-concept. It follows, therefore, that it is important to examine the mat time environment, including specific teaching practices that seek to promote a positive relational climate (for example, group cohesion) and that optimise children's opportunities for participation. The aim of the present research was to explore both teachers' and children's perspectives about their experiences of mat time.

The findings of the two studies in the present research programme suggest that there are significant differences in the ways that children and teachers approach participation within the interactional milieu of mat time. For instance, specific children might resist the authority of the teacher; such behaviour impacts on the attentiveness and participation of the wider peer group. Children's participation appears to be largely influenced by social factors, such as the support of friends and quality of reputation. Teachers must reconsider ways to enhance group cohesion to ensure all children's participation at mat time, as well as query whether instructional questioning is an effective use of mat time for socially or academically vulnerable children's learning and socialisation.

\subsection{Study one findings}

The first study aimed to investigate teachers' reports on their mat time pedagogies and how those pedagogies informed children's participation and interactions. In the first instance, it was important to establish how much time 
children spent on the mat in whole-group activities and what happened during mat time. The reason for this is that the amount of time or type of activity provided could determine how much opportunity children have to participate; for example, a brief mat time or one that requires children to merely listen would offer significantly reduced opportunities for their participation compared with a lengthier, discursive mat time.

It was found that children in year two spend a considerable proportion of their classroom time on the mat. Indeed, for some children this might be approximately one quarter of their classroom day. Logically, this means that there should be adequate time over a day for teachers to enable opportunities for every child's participation at mat time. This does not mean, however, that all children desire an active role or are able to make use of those opportunities.

Alongside the amount of time spent on the mat, a range of activities was facilitated. These feasibly offered a variety of opportunities for children's participation. The most common activities included discussion of rules, reading a book, explaining a learning activity, teaching curriculum knowledge, and acknowledging good work. At the outset, none of these activities overtly indicated that children are able to take any active participatory role because they seemed to be fairly teacher-directed. However, the teachers indicated that they also tended to generally pose a high frequency of questions to the children, enable group discussion, and incorporate some child-centredness and flexibility into their practices.

Such pedagogical approaches are perhaps unsurprising considering the associations that teachers made with children's development. For instance, the teachers were in agreement that mat time enhanced children's academic learning, listening skills, group cohesion, language skills, and social understandings. A significant number also agreed that mat time enhanced children's self-esteem. Indeed, given that the teachers' expressed good support for these outcomes, one 
could assume that they viewed children's engagement at mat time as very desirable, specifically in order to maximise opportunities for children's development.

The teachers were also asked about how they perceived children's engagement and participation. Overall, the majority reported that children enjoy mat time but that they are inattentive. In order to attract children's engagement, the teachers utilised a wide range of strategies. A commonly nominated strategy included placing specific children in close proximity to the teacher. A significant number indicated that they issued reprimands or offered rewards. However, despite such strategies nearly half of the teachers reported that certain children were consistently disruptive. Moreover, the majority of teachers agreed that children's participatory behaviours are generally variable, in that some children consistently dominated, and others consistently observed. In fact, one third of the teachers reported that not all children participated. Therefore, it seems that these specific strategies were not entirely effective at attracting or enabling all children's engagement or active participation.

Despite this, there were some surprising findings about the degree of challenge that the teachers experienced. They were asked to rate how challenging they found it to maintain all children's engagement, ensure the participation of all children, balance the needs of the individual child with those of the group, manage non-compliance, and balance the curriculum with children's interests. Only very small numbers (fewer than 20\%) reported that any of these things were challenging to resolve. Of interest, if teachers indicated that they found one aspect challenging, they also tended to find the rest challenging.

In short, very few teachers found engaging children's interest and ensuring all children's participation challenging, yet a significant number indicated that some children did not participate. Consequently, the subsequent data analyses set out to explore what specific pedagogical factors might boost children's participatory 
behaviours. First, it was thought that if teachers reported that they incorporated children's interests into the mat time that it would mitigate their reports about varying participatory behaviours, such as specific children tending to observe. In other words, an interesting mat time might coax their active participation. However, no significance was found. Nor was there one between teachers' reports of using children's interests and children's attentiveness. In fact, teachers who reported utilising children's interests also did not differ in how challenging they found mat time compared with those who did not report using children's interests. Nevertheless, analysis found a significant correlation between teachers who reported using children's interests and the likelihood that all children participate.

What these findings suggest is that when teachers incorporated children's interests they might have attracted children's desire to participate. However, at the same time, children might have still acted in ways that the teacher interpreted as inattentive. There were disparate participatory behaviours evident, in that specific children behaved in dominating ways whereas others merely observed. Three possibilities exist to account for this phenomenon:

- That the disparate behaviours inhibit certain children's participation while enhancing that of others,

- That children's attentiveness is not linked to their participation,

- That even though the teachers incorporate children's interests, their pedagogical delivery might impact participation negatively (for example the content is interesting, but the activity is disengaging).

With regard to these possibilities, the resulting analyses found that if the teachers reported disparate participation modes, in that certain children dominated, observed, behaved disruptively, or consistently put their hands up, they also tended to report that it is unlikely that all children participate. However, if teachers indicated that children are attentive and enjoy mat time, then it is likely 
that all children participate. Finally, a weak correlation was found between childdirected approaches and the likelihood of all children participating. This could perhaps be because child-directed approaches potentially allow children to influence the topic or activity, thus aligning the mat time to their own interests. As shown previously, links were found between incorporating children's interests and the likelihood that all children participate. In short, utilising children's interests, attracting their attentiveness, and enabling their enjoyment at mat time might enhance the chances that all children participate. Effort made to curb persistently dominating or observing behaviours might also enable participation that is better distributed across the group.

\subsection{Study two findings}

Study Two investigated children's perspectives about mat time. Several studies have determined that the ways that children make sense of the activities, rules, and processes in the setting might be relatively hidden to the world of adults (Corsaro, 1985; Woodrow, 2006). Moreover, there most likely are power dynamics that influence children's participatory behaviours, between the teacher and the students (McLaren, 1999), as well as between children and their peers (Galbraith, 2011). The underpinning premise of participation, used in the present study, encapsulated the notion that influence and social support are integral to children's ability to secure quality participatory roles (Sandberg \& Eriksson, 2011). Study Two investigated the perspectives of 49 children in three year two classrooms in order to explore their impressions of mat time, whether they considered their own participation and engagement important, and whether their peer relations influenced their participation.

Children's impressions of mat time were investigated. The findings showed that half of the children reported neutral feelings about mat time, whereas most of the remaining children indicated that they enjoyed it with the proviso of certain caveats. These caveats commonly included sitting near a friend, getting a good seating position on the mat, and participating in interesting activities. In contrast, 
a disliked aspect included distractions, mostly caused by peers playing around, other children calling out of turn, or peers fidgeting. Several children also seemed concerned about conflict or relational aggression by peers. Approximately two thirds of the children reported that mat time had specific processes, and that most of these related to how the teacher did things. For example, one child described in great detail how the teacher explained a learning concept, then asked children about it and either chose someone to answer in front of the group, or asked the children to share their answer with a partner. Only one third of the children specifically referred to their teacher helping them with their learning at mat time.

The majority of children held an impression that mat time was governed by the teachers' rules, specifically regarding how children should sit and participate. The rules included sitting nicely, not calling out of turn, and paying attention. It seemed that the teachers' explicit rule was that children would not be chosen for turns to participate for rule-infractions, although a small number of children also noted that a teacher might issue spotlighting questions to a child if she thought that he or she was not paying attention. Furthermore, children breaching the rules might be moved to close proximity of the teacher. The positions that were close to the teacher were frequently those that the majority of children viewed as highly desirable; therefore, it was possible that this strategy positively reinforced disruptive behaviours.

The second focus concerned children's active participation. Overall, the children identified three participation modes: these were (1) the whole group when the teacher issued questions or facilitated discussion, (2) small group work that involved sharing ideas with one or two peers, and (3) class-wide competitive games. Most of the children indicated that their personal participation in these activities was important. In fact, only ten children said that it was not important for them to participate. Reasons given in favour of participation included: enjoyment of being involved, benefits to learning, enhanced reputation, possibility of reward, or that it met the teachers' expectations. These responses showed that 
children's participation is motivated by a variety of factors. Nevertheless, even though some of the children said that their personal participation was not important, most of these children also recognised that there were benefits arising from participation.

Paradoxically, for some children, the benefits of participating might have also acted as deterrents. This was particularly true of five of the children who expressed explicit concerns about speaking in front of the wider group, and who indicated that their participation was not important. Their concerns included their own personal shyness or anxiety about having their performance judged by peers. One of them, Masina (Classroom B), suggested that a benefit of participation was that the class might think she was clever; however, she was deterred by the risk of attracting negative judgment instead. Nevertheless, this was also a concern for some of the other children who stated that participation was important. For instance Mia (Classroom B) indicated that she wanted to share her ideas with the class, but she would be embarrassed if she got the wrong answer.

A surprising deterrent to participation involved individualistic instrumental goals, whereby children sought to improve or maintain their situation (as described by Dijkstra et al., 2015). One child, Levi, (Classroom B) had determined that his performance at mat time was of a high academic standard compared to that of his peers; therefore, in avoiding active participation at mat time, he could keep his ideas and knowledge private. He indicated that this preserved his advantage when completing individualised work, such as tests. This contrasted to Loto (Classroom B), who also kept his best ideas private, though he thought that some participation was useful in attracting a positive academic reputation among peers.

Further investigation explored how children's social relations influenced their participation. As well as the reputational issues discussed above, there was considerable evidence to indicate that friends helped each other, such as 
providing information that a friend could share at mat time. Moreover, some children felt more confident about actively participating when they sat next to their friend. Certain children found it easier to work with a friend when the teacher requested small group work on the mat. Overall, the findings strongly indicated that friendship could be highly beneficial to specific children's participation when the friend had good ideas and was focused.

However, social relations did cause issues for some children. For instance, in classroom A, one popular child was concerned that her friends' all wanted to sit next to her on the mat, and were annoyed if they could not do so. By contrast, another child observed the relative popularity of a peer and indicated that it made her feel not very important.

Issues regarding social relations were evident at other times. The first was when teachers' requirement for children to work in pairs necessitated triadic friendships to disband. The result was that one friend was forced to move outside of the group, causing conflict at times. A second scenario was when certain children deliberately distracted their friends. All of the friends in one specific group in Classroom B were complicit in the distracting behaviour; they found the back of the mat in en bloc configurations the best place to play around. However, for other children, distractions were unsolicited and unwanted. Finally, in all three classrooms, certain children gave accounts that indicated that children who experienced social aggression or had low social status might be negatively impacted. For example, Fetu (Classroom B) felt he had to move from his preferred position at the front of the mat if someone "mean" sat next to him.

Small group work might have benefits for socially or academically vulnerable children. For instance, Fetu reported that he liked small group work because he got to "meet new people". These statements are relevant given that his teacher indicated that Fetu was consistently left out when the children were asked to form small working groups at mat time. Areta and Marama (Classroom C) had 
identified that two of the children in their class, Rawiri and Hayley, were consistently left out or needed help with their work, so they asked them to join their working groups. Of interest is the fact that other children, namely Benjamin and Isaac, made particularly derogatory comments about Rawiri and Hayley. This emphasises the importance of children who are willing to assist socially or academically vulnerable children.

A final point that emerged regarding the relational climate at mat time was that of seating position. In fact, most of the children showed a strong preference for a particular seating position on the mat; however, this preference was strongest when children were seated en bloc, as opposed to in a circular configuration. In the en bloc scenario, children preferred the front position because they believed it made them more visible to the teacher or enabled better focus. In short, children believed that front positions in en bloc configurations were beneficial to their learning. However, whereas children expressed a preference for the front, it seemed that only a small number of specific children were able to obtain those positions. Seating position was of such importance to children that many indicated that a poor seating position detracted from their enjoyment of mat time. Finally, the fact that the circle configuration seemed to ameliorate some of the favouritism for certain seating positions should be noted.

\subsection{The interactional milieu at mat time}

Much of the international research about mat time has been situated in circle time, which often has an explicit pedagogical focus on group cohesion and children's self-esteem. Mat time in New Zealand differs because it has a strong focus on instruction and curriculum. However, very few studies are available about this type of mat time. A contribution of the present research is that it has demonstrated that mat time is a common phenomenon in New Zealand classrooms; however, children typically take on varied participation roles as a result of inhibiting and enabling factors inherent within the interactional milieu at mat time. 
As highlighted in Chapter Three, the interactional milieu is the intersection between the official space (i.e. the authority and scripts of the teacher) and the children's social worlds. The present research found that mat time was characterised by competition among the children for limited resources. Furthermore, teachers and children differed in their reports about the social climate, specifically that pertaining to group cohesion. Finally, there appeared to be opposing agendas between teachers and children at times. This means that there are social undercurrents within the interactional milieu that are more complex than is typically evident with surface observation. Moreover, those undercurrents most likely influence the degree of agency and opportunities for participation that individuals can access at mat time.

\subsubsection{Limited resources}

In combining theory associated with children's social worlds and that pertaining to pedagogy, the present research has been able to show that there are limited resources at mat time, and that these can be viewed as a concern within the children's peer group. Limited resources are often those that contribute to children's development and welfare (Charlesworth \& La Freniere, 1983). Furthermore, limited resources are generally a source of social conflict within the group as group members seek to uptake whatever advantages the limited resources offer (Green \& Rechis, 2006). Another important point with regard to limited resources within children's groups is that children's choice of desirable and contested resources may seem trivial to adults; however, their successful acquisition can lead to enhanced dominance or status within the peer group (Hawley, 1999). As such, there is little trivial about them.

One limited resource at mat time was evinced in the high desirability of frontcentre positions in en bloc configurations, despite the fact that these positions were cramped. The children associated the front seating positions with better

affordability to participation and learning, citing the following reasons: they could 
hear or see better, teachers were more likely to call on them for active roles than children sitting elsewhere, and there were fewer disruptions to their learning compared with other places on the mat. Evidence of the front positions enabling less disruption is further supported given that half of the teachers (53\%), reported that they moved specific children to a close proximity near the front in order to enhance their engagement. Overall, these findings indicate that there might be academic advantages to sitting at the front of the mat.

A second limited resource for many of the children included opportunities for active participation. Many teachers gave opportunities for children to influence activity or curriculum at mat time by ensuring that their practices were partially child directed or by incorporating children's interests; therefore, it is unsurprising that taking an active role was important for many children. Moreover, when children are chosen to speak, they share ideas, ask questions, or talk about themselves. Each of these things influences the lesson because the teacher's response is then ideally harmonised to whatever the child has said or asked (Kessler, 1989). As such, it creates a type of feedback loop. This in itself is not a problem; however, a potential problem lies in the notion that it is likely that the children who participate disproportionately more than others, have higher influence over the lesson than their less participatory peers.

Frequently participating children might also be those who are high achieving. Indeed, several studies have found that high-achieving children are also the highest participators in whole group activity (Myhill et al., 2006; Piters, 1995). Certainly, in the present research, some of the children who were most emphatic about the importance of their personal participation were among those who also indicated a high degree of academic confidence. Assumptions can be made that these children were confident because they were comparatively high achieving.

When high-achievers are also the strong participators, their influence might extend to the level of difficulty inherent in the lesson. What this means is that 
teachers might be teaching to the level of understanding suitable for highachieving, high-participating children by primarily responding to those children's ideas and questions, more so than less-achieving, less-participating peers. The needs of low-achieving children might not be catered for. One reason is that they may be unable to accurately follow information pitched to high-achieving children's understandings. A second reason is that opportunities to safely incorporate their ideas or interests are precluded. This means not only potential failure to attract such children's motivation and attention, but also such children have little to no influence at mat time. In fact, Myhill (2002) found that these conditions did not provide an effective learning situation at all, for low achieving children.

Other limited resources included opportunity for a 'good' reputation, as say 'clever' or as someone who won competitive games. Other limited resources were teacher attention, reward and praise. These resources were closely associated with children's academic competence and ability to gain active participatory roles. For instance, in the present research the reputations might have been formed, in part, by the teachers asking many questions. Because strongly participating and high performing children are more likely to answer correctly, they often receive more frequent praise than their peers (Hughes \& Zhang, 2007; Piters, 1995). This, in turn, contributes to such children's status and reputation in the classroom (Donohue, Perry, \& Weinstein, 2003; Mikami et al., 2012).

Another way of putting it is that interactions that are based on questioning not only create a forum for limited resources (praise, positive reputation, reward) but also give certain children an advantage in successfully gaining those resources. In the second instance, the same can be said for specific modes of interaction, such as the competitive games that some of the children described. This is not to say that teachers should never issue rewards or praise. However, there is a strong imperative for teachers to consider who are the most frequent recipients of 
positive acknowledgement and carefully consider what impact this potentially has on the peer group.

So far, it has been proposed that there are several limited resources that children compete for at mat time and that the academically competent children are most likely advantaged; nevertheless, even academically oriented children's strategies to gain these limited resources were of varying effectiveness. Effective strategies require complex interpersonal skills; for instance, an effectively strategic child would possess perceptiveness of the social norms and the relevance of their own behaviour in relation to those norms, as well as being aware of others' agendas and needs (Putallaz \& Sheppard, 1992). Moreover, they have good timing and a certain degree of finesse (Green \& Rechis, 2006; Hawley, 1999).

When children compete for limited resources, a wide range of behaviours are evident that include those that are assertive, coercive, prosocial, and passive (Green \& Rechis, 2006; Green et al., 2008). In the present research prosocial behaviours to gain active participation were evident when children helped their friends by assisting them with 'good' ideas to share. An example of a more coercive strategy includes the one that Whina (Classroom C) adopted; she looked like she was 'slacking off' in order to attract the teacher's attention. It could be that Whina recognised the teacher's conceivable aspiration to attract each child's engagement; by seeming to withdraw that engagement, she not only attracted the teacher's attention but also actualised the possibility of an active role. By contrast, passive behaviours were evident when children withheld attempts to gain a participatory role for fear of judgment or making mistakes.

The strength of a child's relationship and understanding of the social context must be taken into consideration with regard to their active participation. When an individual child possesses an astute appreciation of the ideology and actions expressed through the teacher's practice, they are more likely than their peers with less understanding to be noticeable to the teacher, to be understood by the 
teacher, and to be chosen by the teacher (Churchill, 2003; Cullen, 1991; Vaughn, Vollenweider, Bost, Azria-Evans, \& Snider, 2003; Wentzel, 1993). The sophisticated social understandings that are required are well illustrated by Jack (Classroom C); he deliberately sat next to another child whom the teacher looked at frequently in order to enhance his chances of being chosen by the teacher for an active role.

The overriding implication here is that whereas some children have well developed strategies that fit the social context, other children who have the desire to participate at mat time might be unable to through their comparative lack of understanding of the relevant nuances contained in the social interaction norms. Such children might not possess effective strategies to secure a participatory role. In fact, a few children inferred that they wanted to take part but were unsure how to secure an active participatory role; for instance, this was insinuated in Hunter's (Classroom B) statement that, "I really want to share my idea and be really more involved in the class, because I don't really get to answer questions very much."

The classroom culture plays a pivotal role in the equitable sharing of resources (Green \& Rechis, 2006). This means that teachers need to be aware of which desirable resources are limited and put strategies in place to ensure fairness, especially when there may be educational or social advantages associated with the resource. However, when it came to seating position in the present study, none of the children mentioned strategies that teachers might use to distribute the academic advantages to sitting at the front. For instance, it could be expected that at least some of the children would make mention of such practices, say, teachers requiring children to take turns to sit at the front, if indeed their teachers used such a strategy; yet there were only references to children being moved as a mechanism to curb their disruptive behaviour. In short, if there is an academic or social advantage to sitting at the front, then teachers have an ethical responsibility to ensure that the advantage does not consistently fall to the children who sit there most frequently. 
Similarly, teachers have a responsibility to ensure that participation is distributed fairly and sensitively so that the positive benefits of participation are distributed. In summary, what is evident here is that participation opportunities frequently revolve around individual children responding to the teacher's questions. This is a less than ideal interaction because it rarely enables deep discussion or critical thinking (Cazden, 2001; Myhill et al., 2006; Wells, 1993). Moreover, it means that certain children are able to uptake resources, such as praise, more easily than peers. However, there is also an equity issue, in that certain children have strategic knowledge to enable their participation in this type of activity, and others do not.

Another issue with closed questions when they are issued to the whole group is the differing amount of time that individual students require in order to formulate an answer. When students do not have time to formulate a response to questions, they are immediately precluded from obtaining resources such as praise, rewards, or title of 'winner'. Although it was not something that was raised by participants in the present research, Tobin (1987) posits that teachers should allow 'wait time' and not choose speakers until all children have had adequate time to consider their response. When this happens, more children have an opportunity to formulate more in-depth and critical responses. Of course, this practice would need to work in conjunction with strategies to prevent specific children from calling out of turn. In any case, avoiding overuse of closed questioning in favour of other modes of participation is probably wise, given that Galton et al. (2009) suggest that closed questioning, "is very different from interactive whole-class teaching, which is designed, as with group work, to promote thinking and problem-solving" (p. 134).

The easiest solution would be for the teacher to call on specific children in equal numbers. However, such an approach might cause detriment to certain children who are concerned about their performance or judgment by peers. Therefore, 
teachers must not merely make spaces for less strategic children's participation, but must also address the emotional safety of the social climate and find innovative ways to engage such children's desire to take part. In short, the present findings challenge the notion of "the class" as a single unit, and instead, suggest that whole group situations might advantage socially and academically confident children, while disadvantaging other children. As such, there is a case for observing the most academically or socially vulnerable in the class and ensuring their needs are protected.

\subsubsection{Group cohesion and self-esteem}

Several studies have already examined aspects of teachers' pedagogy and the insider or outsider status attributed to children (Howes, 1990; White \& Kistner, 1992); however, very few have examined the phenomenon from the children's perspectives or examined the roles that children themselves play in excluding specific children. The present research indicated that the peer group is implicit in children's exclusion and low status in the classroom, even with the presence of a teacher, and that this relates directly to the classroom climate.

The classroom climate often sets the scene for the interactions of its members (McGee \& Fraser, 2012). More than that, however, is that the climate directly influences children's learning (Eggen \& Kauchak, 2006). One aspect of a positive classroom climate is group cohesion, identifiable by the degree of support experienced by its members, a sense of togetherness, and shared orientation to tasks (Treadwell et al., 2011). These notions are encapsulated in the New Zealand Curriculum Framework (Ministry of Education, 2007), which proposes that children's competencies should include consideration of how an individual's words and actions can affect those around him or her. There is also reference to children knowing how to create opportunities for others, and knowing how to recognise appropriate times to compete versus appropriate times to collaborate. 
The key competencies guide expectations and desirable outcomes for children's behaviour; however, it seems very unclear how the competencies are enacted when it comes to mat time. This is especially so given some of the disparate behaviours that many teachers had noticed in the present research, such as some children participating more than others, some children behaving disruptively, some children dominating, and some children withdrawing. Nevertheless, teachers predominantly associated mat time with group cohesion. Previous studies that associated mat time with group cohesion tended to foreground the role of specific social activities (Mary, 2012). These activities explicitly promoted cooperation among students and aimed to foster children's relational awareness about each other, which according to Treadwell et al. (2011) are two essential aspects of cohesion. However, this was not the case in the present research, in which the teachers prevalently used mat time for academic activities instead.

In contrast to the teachers, the children's responses were suggestive of greater circumspection about group cohesion. A number of children referred to togetherness $(24 \%)$ in relation the group; however, nearly double that number expressed strong favouritism for individual peers (44\%), whereby they preferred to sit with a friend, or preferred to work with their friends rather than other peers. Furthermore, a small number of children (12\%) spoke of certain peers with outright derision and several children referred to peer-conflicts that occurred at mat time. Furthermore, one child, Sienna (Classroom B), said that she did not know everybody in her class. None of these behaviours appear to subscribe to the shared foci and sense of togetherness that Treadwell et al. (2011) suggest are integral to group cohesion.

As well as the quality of relationships, another aspect that influences the cohesiveness includes the types of activities that are facilitated and what kinds of interactions are promoted. Indeed, McLaren (1999) asks how the practices of classrooms inform the values and behaviours of those who are party to them. In the present research, several children described vying to answer questions or 
participating in competitive games in order to better opponents. When children compete against each other in a highly individualised way, the peer group pays close attention to who succeeds. When an individual has bettered their opponent in such situations or demonstrated superior prowess in a valued activity, he or she might gain or maintain high status. In contrast, less effective competitors might be allocated low status or poor reputation (Fein, 2012). Specifically, the findings of the present research suggest that pedagogies that foster individualistic competition are unlikely to contribute positively to group cohesion.

That mat time fosters group cohesion is doubtful given the degree of judgment about peers that was evident in some children's reports. Some children ascribed negative judgments to peers based on individual children's behaviour or academic performances. Howes (1990) suggests that these judgments can become entrenched within the peer group, resulting in significant ramifications for individual children and group cohesion. She states that, "with increasing time in a peer group, a child's reputation may be better known by others than the child's actual behaviour" (p. 328). When a child's reputation becomes entrenched, there could be implications for his or her status within the peer group, identity, and selfconcept. For some children in the present research the consequences were generally positive; for instance, having reputations as academically formidable opponents in games. In contrast, consequences for other children were negative because of their poor academic reputation.

There are concerning consequences for children who are relegated to low status. Indeed, the poor reputations and low desirability attributed to specific children might have negative ramifications for their self-esteem. Garandeau, Lee, and Salmivalli (2014) assert that people generally consider social hierarchies to be natural aspect of being human; however, it is now well known that status inequalities among children are increasingly associated with negative consequences, especially as children age. Such consequences include peer victimisation and impaired peer relationships (Bukowski et al., 2011). Moreover, 
children's self-esteem is integrally tied to cognitive competence and peer acceptance. An individual's self-esteem is partly informed by others' appraisals of him or her (Rosen \& Patterson, 2011). Despite this, $65 \%$ of the teachers in the present research indicated that mat time contributes to children's self-esteem.

A further point is that the ways in which teacher feedback is issued might also contribute to social divisiveness. When a teacher issues positive feedback, the child is likely to be more positively viewed by peers (Donohue, Perry \& Weinstein, 2003). This effect is vey strong; for example, Mikami et al. (2012) studied 490 American children from kindergarten to fourth grade and used classroom observation and sociometric testing to examine links between teacher feedback and social hierarchy. They found that when teachers issued frequent positive comments to certain children based on academic talent, the social hierarchy was rigid; children showed preference for fewer peers. Conversely, where teachers demonstrated less favouritism, the children's social hierarchy was far less rigid and children changed their preferences for particular peers throughout the year (Mikami et al., 2012). Such findings suggest that the nature and distribution of feedback directly impacts the social cohesion of the group.

So far, this chapter has been critical as to whether mat time has achieved group cohesion; however, it is equally important to acknowledge the positive elements relating to cohesion that were evident. In the first instance, $24 \%$ of the children said that they enjoyed aspects of mat time that promoted togetherness. This often meant that these children enjoyed the sense of the whole group being together or that they liked the opportunity to interact with others with whom they typically would not interact. Furthermore, two children from Classroom $\mathrm{C}$ appeared to be concerned about the low status and poor academic reputations of two of their peers. These two children reported that they made a conscious effort to include the low status children. Prosocial behaviours such as these that enhance a sense of togetherness need to be reinforced if there is to be any effect on group cohesion. Indeed, Moss and Wilson (1998) targeted the relational climate at mat time, by 
encouraging children to choose different partners; as a consequence, they reported positive improvements to the sense of cohesion felt among the group.

In short, the findings of the present research suggest that mat time has potential to enhance group cohesion and that some children and teachers value this as an outcome. Nevertheless, there seems to be a focus on interactions that promote individualised competition and that contribute to social division among the children's peer group. With these practices, mat time is unlikely to achieve the group unity and positive social relations that are key aspects of group cohesion (Treadwell et al., 2011). Moreover, the mat time practices could even be costly to specific children's self-concept. This is particularly so in cases in which the peer group ascribes poor academic, behavioural, and social reputations, and whereby individuals experience frequent exclusion when small groups are called for.

\subsubsection{Differing agendas}

The literature about whole-class pedagogy almost exclusively reports the academic and social elements from the viewpoints of adults. In contrast, the present research balanced the perspectives of adults with those of children. Not only does it demonstrate that a single class comprises members with varying reputation and status, but that the agendas of its members influence interactions. The efficacy of whole group teaching is questioned again, in that the present research clearly shows that children's goals, motivations, and needs are complex and varied, yet the teacher logically, has less capacity to respond sensitively in whole-class situations than in one-on-one interactions. Moreover, specific children are likely to appropriate teachers' pedagogical structures in order to achieve their own agendas.

In the present study, teachers and children described differing agendas. Broadly, teachers identified a range of outcomes resulting from mat time that related to teacher agenda. These included children's enhanced cognitive knowledge (70\%), social understandings (83\%), language (86\%) and listening skills (92\%). Children 
were not asked specifically about their goals or desired outcomes; however, the analysis showed that children held at least two kinds of goals, each of which had synergy with Corsaro's (2012) observations of children's goals. He posited that there are two common goals arising from children's interactions; the first type is egoistic, which is individualised, aimed at the attainment of individual desires, and is characterised by winners and losers. One example includes Benjamin's (Classroom C) selectivity about which partner to choose for enhanced performance in pair discussion. Another is Quentin's (Classroom B) desire to come first in the game, Top Cat. The second kind of goal is prosocial whereby the primary aim is to maintain the quality of affiliations and relationships within the group, rather than promote individual interests; for instance, Areta and Marama (Classroom C) included two children in their small group work whom they believed needed social and academic support.

Alongside Corsaro's (2012) goals, children's desired outcomes could be loosely orientated to McClelland's (1961) Three Needs, namely, personal achievement, affiliation, and status or recognition; for instance, Cooper (Classroom B) had a strong need to affiliate with his friends, so he sat at the back of the mat and played around with them. However, this need for affiliation was not stronger than his desire for personal achievement; therefore, he moved to the front of the mat if he wanted to understand something better. However, McClelland's work was about adults and did not encompass the power differential existing between children and teachers. In the present study, several children indicated that their participation was important so that the teacher knew that their behaviour was on task. This view very tentatively aligns with McClelland's notion of the need for recognition. Nevertheless, a stronger association perhaps aligns such an agenda with Dijkstra et al.'s (2015) assertion that some children are motivated by normative goals, in that they do something because they are expected to.

Children's orientation to specific goals or needs might influence the ways in which they take part at mat time. For instance, there was a small number of confident 
children (12\%) who clearly relished the chance to compete against their peers. What each of these children had in common in their interview comments were: the importance each placed on participation, confidence in their own academic abilities, and a tendency to judge their peers' abilities. For such children, their participation might be driven by egoistic goals, such as a desire for status and recognition. This certainly seemed the case for Loto, Quentin (Classroom B), and Benjamin (Classroom C), who referred to getting rewards or acknowledgement for participating in line with the teachers' expectations. However, Loto and Quentin also focused on their own learning, as did Cooper (Classroom B) and Isaac (Classroom B); this meant that they asked questions of the teacher when they did not understand something. Nevertheless, with the exception of Cooper, the children who relished the performance aspect of mat time also referred to affiliation goals and outcomes such as involvement, friendship or togetherness.

The issue of peer-appraisals and comparisons might have triggered an agenda to 'save face' in the cases where children were reticent about taking part. Some children might choose to avoid participation if they believe they might face failure and encounter negative appraisals by observers (Rosen \& Patterson, 2011). Their concerns about saying the 'wrong thing' on the mat, or being judged by peers indicates that, for these children at least, the emotional climate might not have been secure enough for them to risk making errors. Given that there are links between peers' perceptions of other children's academic performance and children's acceptability to the peer group (Asher \& McDonald, 2010; Donohue et al., 2003; Gest et al., 2005; Mikami et al., 2012), this must be taken seriously. Nonetheless, half of the children said that it was more important to try rather than to be right, which potentially reflects the teachers' agenda.

Further differences in agenda between children and teachers were evident when children appropriated the teachers' interactional structures to enact peer concerns. A clear example was when teachers asked children to form pairs in order to discuss a topic, presumably with the intent to enhance children's learning 
and understanding. However, this practice impacted children's affiliative agendas when triadic friendship groups were involved. This was because one friend had to partner with someone else, outside of the triadic friendship. Whereas the teachers' agenda was most likely learning oriented, the children's agenda meant that the practice created relational competition or tension with some friendships. When the structure of the mat requires a friend to be excluded, social dissonance and distraction might be caused that most likely detracts children's attention from the actual mat time lesson.

Similarly, differing agendas were evident with regard to rules. Whereas teachers see rules as essential for creating and maintaining order and a positive learning environment (McGee \& Fraser, 2012), certain children might view some of the rules as practices to be resisted or subverted. In particular, rules about fidgeting and sitting nicely relate directly to adult control exercised over children's bodies. Fingerson (2011) asserts that the body is central to power relations between children and adults in school, and relates directly to children's agency. McLaren (1999) also agrees that power relations are embodied physically. In the case of fidgeting and sitting nicely, children's rights over their bodies are superseded by the teacher's need to impose order on the mat. Another often-cited rule in the present research was children not talking out of turn; the rule called on children to raise their hands and wait for the teacher to call on them to speak. The teacher uses his or her authority to choose who may speak and who must listen (Gallas, 1992; Rubenstein Reich, 1994). Again, the children's desire to use their bodies to speak is constrained by teachers' comparative power.

Children will breach the rules for a wide range of reasons (Cook et al., 2014; Milman, 2009). Of particular interest to the present study was the notion that some children resist and challenge the rules as a way to reclaim a level of control over their lives and their bodies (see Corsaro, 1985). Hobday-Kusch and McVittie (2002) support this view; they studied Canadian children in first and second grade. Their observations found that specific children's attempts to disrupt the 
rules were a form of power negotiation. In particular, they noted that even though teachers exerted their authority, some children resisted by withdrawing attention. Similar findings were reported by Rubenstein Reich (1994) who asserted that:

There was a possibility for the children to oppose, protest against and refrain from wholly complying with the demands raised in connection with circle time. Common ways of protesting were leaving the circle,...demonstratively not participating in activities, refraining from participation in more subtle ways, verbally protesting against instructions and obeying them anyway. (p. 56)

Unlike the mat times in Rubenstein Reich's (1994), the children in the present study were not allowed to leave the mat; therefore, protest most likely occurred by withdrawing attention. In fact, prior to interviewing children, I remember one child's statement akin to the sentiment that the teacher made his body go to the mat, but he just took his mind away.

Withdrawal is not the only method of resistance, however. Other children "act in ways that are sometimes considered pathological" (Hobday-Kusch \& McVittie, 2002, p. 200). The latter group utilised a range of strategies, such as employing peer-relevant humour to disrupt proceedings and gain control. According to the children in Study Two, disruptive peer humour included playing around on the mat. Such behaviour risks the chance of attracting reprimands or punishment issued by the teacher. In the present research this was evinced when several children referred to 'getting into trouble' for playing around or otherwise behaving disruptively.

Children often flirt with the fantasy of danger with the aim of avoiding being captured or harmed. This flirtation with danger is commonly evinced in the fantasy play of children whereby themes of monsters or baddies are played out (Corsaro, 2012); however, it is possible that similar approach-avoidance plays out 
in the classroom, with regard to adult authority. In other words, specific children might seek to mock and avoid adult authority.

Disruptive behaviour might also serve to enhance the specific group of children's sense of togetherness as a sub-group of the wider class (see Galbraith, 2011). This was particularly evident with children seeking to affiliate with specific peers. Togetherness requires a group of individuals to form a common agenda, and agreement on shared action (De Haan, \& Singer, 2001). The shared action, in turn, can serve to maintain friendships, amplify feelings of competence, and maintain or recreate subculture within the peer group (Cosaro \& Molinari, 2008; Lambert et al., 2013; Van Oers \& Hännikäinen, 2001).

Other behaviours also communicated dissonance in agenda between teachers and specific children. Teachers indicated that they issued a high frequency of questions to children. This was done, presumably, as a mechanism to encourage all children to mentally attend to the topic, or as a mechanism for teaching facts. Nevertheless, as discussed previously, certain children took a competitive approach to answering. Indeed, the second most common behaviour that the children in Study Two referred to as disliked was peers calling out of turn, which surely breached the teachers' expectations that one person would speak at a time. Moreover, calling out can interfere with other children's learning (Vargo, 2008). For example, interference could be particularly relevant when the teacher has asked a closed question and has paused for children to formulate an answer. When one child then calls out an answer, particularly a correct one, other children are denied the opportunity to follow their personal line of reasoning. If specific children call out frequently, other children might interpret the behaviour as a bid to assume more than a fair share of turns to actively participate, and therefore, view it as an attempt for dominance over peers. What this does do, however, is illustrate that official space and children's worlds each contain a diverging agenda with regard to questions and how they are answered. 
When the agendas of teachers clash with those of specific children, teachers might be required to guide the children's behaviour toward that, which the teacher prefers. Nevertheless, despite the children's references to disruptive behaviour and the possibility of peers being 'told off', surprisingly few teachers in Study One indicated that they utilised strategies such as reprimands. Similarly, fewer than a quarter of the teachers indicated that they issued strategies that could be perceived as punishments for infractions, such as writing a child's name on the board or removing children from the mat area. What was more common was the use of placing a child in close proximity to the teacher and issuing rewards to encourage children's on-task behaviours. This potentially created a dissonance for specific children in en bloc configurations, given that the front seating positions were highly desirable; therefore, the proximity to the teacher could be perceived to be a reward. As such, it could be similar to other cases cited in studies (Cook et al., 2014; Vargo, 2008) whereby teachers unwittingly rewarded disruptive behaviour. The inference, here, is that some children might have been able to appropriate teachers' agenda that are communicated through guidance structures in order to obtain certain limited resources.

One example of a child disrupting a teacher's attempts at guiding behaviour was seen in Quentin's (Classroom B) account of being in trouble with the teacher for acting disruptively. In his account, he was going to be required to tidy the classroom. Subsequently, he ensured that he behaved more in line with his teacher's agenda. In doing this, he not only avoided punishment, but also was rewarded, in that he was allowed to leave the mat before others. Regardless, Quentin indicated that he still persisted in sitting at the back of the mat so that he could better play around with his friends, rather than attend to the teacher. This suggests that the threat of punishment was not effective in engendering his ontask behaviour.

Not only do the children within a single group differ in their agendas, but it also seems that their agendas are multifaceted and complex. Furthermore, they most 
likely change according to the situation (Ojanen et al., 2007). Even so, in cases in which individual children are motivated by strong egoistic goals, or need for power and status, teachers must carefully reflect on whether they should support such agendas. If unchecked, such children could take on significantly more agency and influence than that which is afforded to their peers. It could be that, if cohesion is valued, then, egoistically driven children should be assisted to behave more collaboratively and less individualistically. Of interest, in the present research, 19\% of the teachers in Study Two indicated that balancing individual children's needs with those of the wider group was challenging. Brown and Thomson (2000) suggest that one strategy could be to encourage children to cooperate in achieving shared goals as opposed to practices that foster individualistic goals, as IRF-type interactions appear to do.

There is some evidence to indicate that activity, which is designed to promote cooperation and collaboration rather than individualised performances, might ameliorate at least some of the divisive effects of competing agendas at mat time. For instance, Mary (2012) observed the effect of games that required the children to collaborate with each other. Over a year, the games led the children to relate to non-friends better; they reported stronger understandings of peers, better acceptance of them, and behaved more inclusively towards them. Similarly, Finlinson, Austin, and Pfister (2006) analysed the difference in behaviour of 4 and 5 year old children in Utah when they took part in a range of games. Some were cooperative games, whereas others promoted individualised performance and competitive behaviour, in that they allowed only one child to be the winner. The children exhibited significantly more positive behaviours during the cooperative games compared to those that were exhibited during the competitive games.

\subsubsection{Why did children participate or not?}

In summary, there appeared to be several reasons for children's varied participation at mat time. Those, who expressed a desire to participate, did so as a 
result of a range of motivations that they brought to the interactional milieu. Several theoretical frameworks can account for the different motivations. First, McClelland's (1961) Three needs theory posits that individuals seek affiliation, personal achievement, or power. Similarly, Buhrmester (1996) suggested that motivations are driven by either affiliation or a need for power and agency. By contrast, Djikstra et al. (2015) asserted that individuals might be motivated by either immediate gratification, by improving their situation, or by the expectations of others. Each of these theories has merit when it comes to children's motivations for participation at mat time.

These theories represent a wide array of good reasons for participation at mat time, and yet it was evident in the present research that specific children did not participate. The work of Ryan and Deci (2000) offers a plausible explanation. They suggested that human beings are, by default, "curious, vital, and self-motivated" (p. 68); however, an insensitively attuned social context undermines that potential. According to Ryan and Deci, people have three psychological needs: autonomy, competence, social relatedness. When the three needs are fulfilled, an individual is motivated. By contrast, when the needs are thwarted, an individual's motivation, confidence, and well-being are negatively impacted on.

In the case of mat time, individual children's participation seemed - in part related to the extent to which he or she experienced autonomy, competence, or social relatedness. For example, some children reported that mat time was boring to them, or that activities of interest were offered infrequently. Notwithstanding, their attendance at the mat was a requirement. Accordingly, they had little autonomy in the situation. It is of no surprise that some children acted out. Similarly, other children were concerned about how they would be perceived academically, or were worried about their performance. Correspondingly, their need for competence was unlikely to be fulfilled. Notwithstanding, the converse was true in each case for children who did have these needs met; they were likely to participate. Finally, social-relatedness had a strong bearing over specific 
children's participation, in that it was associated with inclusion, assistance, and influence.

With regard to children's needs, a further, major, facet of the interactional milieu that impacted on participation was how the teachers structured children's opportunities for active involvement. To put it simply, the teachers determined the kind of activity that the children participated in, whether it was an explicitly competitive game or whether it was a small group discussion. The different types of activity appeared to appeal to Ryan and Deci's (2000) Three psychological needs theory in different ways. It is suggested here that an ideal structure to meet many children's psychological needs of autonomy, competence, and social relatedness might be met more effectively through the use of small groups rather than the whole group scenario. The following section elaborates on this notion.

\subsection{A case for distributing participation}

Teachers play a critical role in establishing the social climate within classrooms, including during activities that bring the group of children together into one space, such as at mat time. McLaren (1999) asked two questions that are pertinent to mat time. The first is whose interests does the practice serve? In the case of mat time, it would seem that one group that it serves the most is the teachers, who can articulate a message to the whole class quickly. Another group that is served well appear to be children who can take on high-participatory roles with confidence in their ability. It is timely to consider how mat time can better serve children who struggle to actively participate.

\subsubsection{Using children's interests to encourage participation}

The present research found that even when children indicated that they enjoyed mat time, their enjoyment was dependent on caveats such as the type of activity and whether they were able to participate in it. Basically, enjoyable activity might incentivise certain children's desire to participate. A significant association was found between teachers' reports that they incorporated children's interests and 
their agreement that all children participate. Moreover, there was also a significant association found between teachers' reports of children's attentiveness and enjoyment with the likelihood of all children participating.

Children who are engaged in learning activities might be likely to experience higher levels of achievement compared to when they are not engaged; this in turn informs their self-perception (Chen, Darst, \& Pangrazi, 2001; Hidi \& Renninger, 2006). Typically, engagement has been associated with interest, in that when an activity is appealing to students, it motivates them to engage with it, and therefore participate (Chen et al., 2001). In short, when teachers aim to engage children's interest, a pathway to engagement is cleaved more easily; engagement is an important aspect of learning and self-concept. In fact, Hidi and Renninger (2006) state that:

Even though interest has been recognised as an important condition for learning, educators ...do not have a clear understanding of their role in helping students develop interest. In fact, teachers often think that students either have or do not have interest, and might not recognise that they could make a significant difference to the development of students' academic interest. (p. 111)

Moreover, the careful inclusion of interests can also lift the status of low-achieving children. When activities promote a very wide range of interests, children who struggle academically might discover a niche whereby what they know and can do is affirmed in front of the wider group. In fact Gest and Rodkin (2011) suggest that:

Teachers who provide a particularly stimulating classroom environment may provide a context in which a wider range of student talents or competencies provide routes to peers status, 
thereby attenuating the association between prosocial behaviour and status. (p. 295)

Nevertheless, even when teachers use interesting material, children each still require different levels and types of support when faced with a variety of information (Eggen \& Kauchak, 2006). They possess a variety of working theories, learning strategies, and understandings (Hargreaves, 2013); therefore, when teachers are required to formally instruct on academic knowledge, they are unlikely to cater for the wide range of requirements of individuals across the group even when they attempt to make the content interesting. Rather than allow certain children to consistently demonstrate their academic dominance at these times, it seems logical to differentiate students into smaller groups for mat time instruction, and therefore adjust that instruction accordingly to be responsive to the children's learning. This suggestion is given further weight given the growing number of studies that describe whole group instruction for young children as ineffective (Abd-Kadir \& Hardman, 2007; Cazden, 2001; Coles, 2005; Nassaji \& Wells, 2000). That is not to say that mat time cannot be used for other purposes such as discussion, cooperative learning, or games.

\subsubsection{Distributed participation to increase engagement}

Even though the provision of interesting tasks is an important consideration at mat time, it is equally important to note that there are benefits in children being able to become engaged in activities that are not enjoyable to them; for example, tidying one's room (Joussemet et al., 2004). This is particularly relevant in the context of the classroom, where children are sometimes required to take part in activities that they do not enjoy. In fact, less than half of the children stated that they enjoyed mat time. Furthermore, the findings of Study One suggested that regardless of whether teachers reported incorporating children's interests or not, they still generally tended to rate children's attentiveness as poor. These differences might be accounted for if teachers and children focus on different aspects of interest during mat time activities; for instance, it is possible that 
teachers considered the content or topic that the activity promoted, whereas the children considered the actual activity itself.

Indeed, Hong (1995) and Eirich (2006) each examined the ways that teachers incorporated children's interests through ethnographic observation. In each case, teachers took considerable care to identify topics of interest to specific children and relate them to the mat time content. In contrast, Cefai et al. (2013) found that children stated that they enjoyed specific activities. Similarly, in the present research, children commonly cited enjoyable activities, such as games. Other specific activities that children referred to included reading books, doing experiments, and small-group work. Nevertheless, some of these activities were those that teachers indicated were facilitated comparatively infrequently compared to other activities.

The type of activity provided might be a critically important factor in securing children's engagement; specifically, that which allows for a wide range of children to participate might be most desirable. Previous studies have argued that children's engagement has consanguinity with their ability to actively participate. Children's engagement arising from enjoyable activity was confirmed through the classroom observations of DiCarlo, Pierce, Baumgartner, Harris, and Ota (2012) in 40 North American preschools. They found that one of the strongest predictors of children's engagement was the provision of interesting materials to all children to enable them to take part in learning activity. Similarly, Wood et al. (2009) found that the inclusion of response cards ${ }^{11}$ at mat time in junior classrooms enhanced children's engagement, and that children reported they enjoyed using them. What these things each have in common is that they distributed participation in enjoyable and interesting ways to children. Not only that, but children participated as part of a group, rather than individually.

11 The response cards were a pack of cards given to every child. Each card had a response on it. The children were required to find the correct card in response to questions posed by the teacher. 
The findings of several other studies (such as Cefai et al., 2013; DiCarlo, et al., 2012; Mary, 2012) also suggest that, while the activity itself might be important, of equal importance is how participation in the activity is distributed across the group. In other words, it is important that all children are able to participate and that this will enhance children's enjoyment. This idea is supported through the findings of the present research, which associated teachers' agreement that all children participated with attentiveness and enjoyment. It seems that listening and observing might not attract many of the children's interest or enjoyment, but taking an active part in shared activity does.

\subsubsection{Distributing participation through small groups}

As stated previously, the children identified three main forms of active participation at mat time. These included whole-group competitive games, wholegroup discussion and instruction, and small-group work. The latter practice was characterised by the teacher asking children to form pairs or small groups. One of the obvious factors that make this practice appealing is that it distributes participation; specifically, several children can speak at once. Furthermore, Myhill et al. (2006) suggest the use of small groups and pair-share for enabling safer and more frequent participation roles for low-achieving children. This might also be true for the children in the present research who characterised themselves as having shy or anxious temperaments. Furthermore, Myhill et al. (2006) suggest that even short bursts of small group talk during whole-group situations assist children in participating and maintaining engagement.

Not only might the use of small groups assist with children's involvement in the topic, but it might also enhance the actual interactions. As stated previously, whole group interactions on the mat are notoriously dominated by teacher-talk and teacher-questions that require children to merely recall information. In fact, McGee reported that teachers asked closed questions approximately every 12 to 30 seconds and waited less than a second before calling for answers (McGee \& 
Fraser, 2012). Myhill et al. (2006) report similar findings. Furthermore, they assert that:

From the 54 lessons observed and recorded, there were only 20 examples of children asking questions, meaning that in the majority of lessons observed there were no questions from children at all. Children's voices in general and their questioning voices in particular are rather silent in our whole class 'interactive' teaching. (p. 79)

A well-posed question or challenge, which underpins discussion is more likely to engender in-depth thinking than a poorly posed question. Yet, because teachers are required to facilitate the agendas of a large number of children in whole group situations, it can be exceptionally difficult to apply the rich questions that engage children's higher order thinking skills. Similarly, teachers' ability to make space for children's questions is limited in whole group situations because he or she must work at maintaining the group's attention. (Cazden, 2001; Myhill et al., 2006). In short, some of the benefits of using small groups skillfully for interaction are that they can enhance the depth of discussion and enable children to ask questions more readily.

\subsubsection{Teachers' selection of small groups}

It cannot be claimed that small group or pair interactions at mat time are without issues, especially when children self-select their groups. This was the case in the present research, where children predominantly referred to selecting their groups. A state of en dehors was evident, in that the collaborative nature of small groups was promoted as part of the official space; however, some children were particularly averse to engaging in shared activities with certain children who were attributed with poor social, behavioural, or academic reputations. Children who experience persistently low status are likely to experience difficulty in accessing participatory roles because of their outsider position, and could 
ultimately become disengaged in their learning. A lack of acceptance is also indicative of later maltreatment by peers (Buhs et al., 2006). The children who were singled out for poor reputations most probably struggled academically, with some peers stating that such children were lazy or did not think, and others indicating that they needed help.

In these cases, teacher-assigned groups might be more effective. They could have the benefit of bringing children alongside others who they would not ordinarily interact with, separating children who might distract each other, and putting children together if the special support of a friend is required. Indeed, according to Brown and Thomson (2000), children often use reputation and social status as criteria for selecting members to work with when asked to form small groups; therefore, they recommend that teachers select the groups. Despite this, childselected groups may be the most common method that teachers use to formulate small groups. They assert:

Student-selected groups often have powerful social agendas that take up their time and attention and results in much 'off-task' behaviour. It reinforces and stabilises social cliques in the class. Those students who have difficulty in interacting socially or tend to be loners and need very much to be included are left out of the student-selected social groupings, adding to their feelings of isolation. (p. 64)

Teacher-selected groups would also prevent high-performing students from joining together to enhance their own academic advantage and ability to compete. According to Gest and Rodkin (2011), academically similar students often group together; this is known as academic-homophily. What is known is that teacher differentiation, based on academic ability, can result in certain privileges for highachieving children. For example, Hughes and Zhang (2007) examined the children's academic ability and peer perceptions of 291 children in First Grade in 
Texas schools. They found that when children were in highly differentiated classrooms, children across the class reported low self-perceptions of cognitive ability. However, those in high achieving groups received more teacher praise, educational opportunities, and special privileges, compared to their less-achieving peers.

Homophily of any kind operates in several patterns. Because people group together with very similar characteristics, there are implications for the diversity of attitudes and knowledge that that they are exposed to, and the interactions that they experience. Very homophilous groups risk losing the rich social knowledge that can gained from interacting in heterogenous groups; therefore, they are susceptible to 'group think' (McPherson, Smith-Lovin, \& Cook, 2001). However, in the specific context of mat time, academically-homophilous groups of highachieving children would receive benefits disproportionate to their peers if there is potential to receive a reward of some description, whether it is teacher acknowledgement or enhanced reputation within the peer group.

Overall, teachers who promote new friendships when creating groups have a less pronounced hierarchy within the children's peer group. Furthermore, separating disruptive friendships seems to promote greater liking across the peer group. A third benefit is that classrooms that encourage groups comprised of academically diverse students are associated with a more egalitarian social hierarchy than those that use academically-homophilous groups (Gest \& Rodkin, 2011).

\subsubsection{Collaborative small group interactions}

There is still propensity for specific children to be barred from taking active roles even within small groups. It was not within the scope of the present research to investigate this; however, Hynds' (2000) research into teachers' collaborative projects reported one vignette in which two children excluded a peer during the small group discussion. Not only that, but they directed relational aggression toward the peer. Brown and Thomson (2000) indicate that teachers must put in 
place considerable groundwork before small groups behave cooperatively. They suggest that teachers must outline a clear culture of collaboration whereby listening and inclusion are explicitly discussed and encouraged. Other strategies include grouping children who are likely to work well together, allocating time for every speaker, and managing the roles within the group (for example, having one person be the group leader, another be the person who reports the conclusions of the discussion or activity to the teacher or class). It is conceivable that having the teacher moving around the groups would also assist. Finally, there might be benefits to all children's desirability as working partners when teachers can promote specific aspects of each individual child's competence.

\subsubsection{Recommendations to enhance children's participation at mat time}

Children's participation at mat time varies. Reasons include specific children's concerns about making mistakes in front of the peer group or low reputation. Other children might be likely to perform less well in the competitive interactional pattern of IRF sequences or other mat time contests. Children might participate less often when they are not interested in the activity, or fail to gain an active role. Specific structural factors might impact this such as seating position, ability to see and hear the teacher well, the degree of disruptions by peers, and the agendas of friends.

In these cases, teachers must go further than legitimate assumed practices or conventions. Moreover, he or she must never assume that the social relations among children are unchangeable or that the teacher has no hand or no responsibility in children's social worlds. The role of the teacher is aptly surmised in McLaren's (1999) statement that, "the teacher performs a social function that is never innocent. There is no neutral" (p. 253).

Farmer et al. (2011) describe the role of the teacher as the "invisible hand" (p. 248) when it comes to social opportunities in the classroom. They assert that, "teachers are in a position to develop, guide, and direct the classroom as a society" 
(p. 249). As such, teachers are expected to take on a deliberate and conscious role in shaping children's relationships with each other. This means that they must find and communicate the social value of all children, not just those with the best goodness-of-fit. Broadly, teachers' relationships to each child matters, because the social climate will be partway informed by the quality of these relationships. Teachers are also able to foster opportunities for children to socialise with those whom they typically would not gravitate to. Finally, consideration given to the structures of the classroom and the ways in which they produce children's hierarchies is essential.

Overall, the role of the teacher in fostering a positive social climate is of critical importance, in that the quality of an individual child's peer relationships unequivocally influences the quality of their learning (Farmer et al., 2011; Hartup, 1996; Kindermann, 2007). Moreover, it has an important impact on individual children's self-concept and social skills (Bukowski et al., 2011; Howes, 1983). With this in mind, the official practices of mat time inform its social and interactional climate, which, in turn, influences individual children's participation.

Relationships, peer culture, and the norms of official space most likely interplay in ways that mean that each classroom is its own unique context. As such, the efficacy of mat time would need to be evaluated by how well the strategies are consanguine with the specific context. Indeed, each context is likely to require its own catalogue of nuanced strategies in order to encourage each child's participation. Nevertheless, a range of broad recommendations is made that could mitigate the issues which prevent some children from participating. These include:

- A carefully constructed social climate that places value on inclusion and collaboration. 
- Use of a circle configuration where possible or ensuring that different children are given opportunity to sit near the teacher.

- Inclusion of all children's interests and validation of competencies.

- Ensuring that all children can see and hear well.

- Minimising IRF-style interactions and other practices that promote individualised competition.

- Having at least some mat times in which cooperative games or activity are facilitated that likely foster group cohesion.

- Carefully considered distribution of feedback or acknowledgement.

In addition, the use of small groups can be used to distribute participation; however, teachers should select the members of groups in order to:

- Prevent certain children being excluded.

- Manage triadic friendship groups.

- Partner children who require additional support with children who are likely to support them.

- Manage disruptive or off-task behaviour.

In addition, the different group roles should be managed; for example ensuing that children take equitable turns to be speaker and listener.

\subsection{Limitations and future directions}

The research aimed to present a broad overview of participation at mat time. However, it should be noted that caution must be exercised when generalising the findings. Participation is very context specific. Boylan (2010) asserts that participation can only be deeply understood in relation to the specific classroom ecology. Moreover, it should acknowledge the specific social practices that determine whether an individual's participation is supported or resisted, required or voluntary. Specificity of context is also an important consideration given that children's peer cultures often have nuances that relate to the setting (Corsaro, 1985). Indeed, even social status rests on traits that are valued by the individual 
within the specific context, which means that what is valued by one group might be less valued by another (Fein, 2012).

Some of the most significant limitations of the study occur in omissions in the survey. For example, there would have been benefit in defining specific terms that have multifaceted elements; such as group cohesion. Furthermore, the survey failed to ask teachers about the seating configuration that they used, or about the size of the class. It might also have been beneficial to ask about strategies that teachers used specifically to coax reluctant contributors or curb children who dominated at mat time. Some teachers might use cold calling as a way to ensure that low-participatory children take part; however, the survey did not ask teachers about this. Cold calling refers to calling on an individual irrespective of whether they want to participate or not. Finally, at the time of developing the survey, I did not realise that pair-share and small-group work would be so important to children, and therefore, teachers were not asked about it. Overall, the survey could have been enhanced by the use of 4-point continua, as opposed to 5point continua; this would have prevented situations in which teachers predominantly chose the mid-point. Overall, the findings of both studies are limited owing to the fact that the data are based on teachers' and children's perceptions. It stands that perceptions could be incorrect.

There are several areas that require further research. For instance, in Study Two, many children indicated that the front seats afforded greater likelihood of being chosen for active participation. Future research could verify the accuracy of those perceptions. Further research is required about the synergy and dissonance between children's and teachers' goals. Such research would make a valuable contribution to the body of literature concerned with classroom pedagogy. The present study did not examine the experiences of children with inclusive education requirements. However, it seems likely that such children might have unique experiences regarding participation and status at mat time. To date, it is not known whether teachers' mat time practices achieve their aims. There is some 
literature to suggest that it is a poor strategy for teaching low-achieving children. Studies that aim to establish the efficacy of mat time as a learning context would be beneficial. Finally, there would be merit in research that seeks to assist children who are fearful of being wrong or being judged to build their resilience and their participation repertoires during mat time activity.

\subsection{Conclusions}

Despite many teachers' assertion that mat time in New Zealand year two classrooms enhances group cohesion and self-esteem, there is considerable doubt. When interviewed, children placed emphasis on competitive games and individualised responses to teachers' questions. Furthermore, many demonstrated favouritism for specific peers and it was evident that certain children had low social status within the group. Nevertheless, previous studies show that mat time can enhance group cohesion and children's self esteem; however, specifically designed activities are required for this purpose. The teachers and children in the present research infrequently referred to such activities.

Children bring multiple agendas to the mat, socially and academically. Given that such agendas can motivate individuals in various ways, the role of the teacher is made very complex. Nevertheless, few teachers reported that they found balancing individuals' needs against those of the group very challenging. Overall, it was found that teachers and children share some aims and desired outcomes for mat time. Nevertheless, some differences exist between teachers and children; these differences are associated with individual children's status and inclusion. For instance, two children operated from egoistic goals in keeping information to themselves rather than the more prosocial behaviour of sharing it with peers. Another example was seen in the very high priority that children placed on getting a front seat in en bloc situations. 
Confident children relished the performance aspect of mat time. Previous literature suggests that such children might be high achieving and might dominate turns to speak. It is possible that such children can influence content of discussion and level of instruction. Given that the teachers allowed reasonable opportunities for children to influence activity and content at mat time, any bids by specific children to dominate these opportunities should be viewed with concern. Successfully dominating such opportunities would allow certain children to prioritise their own interests and strengths.

Children enjoy mat time when they can actively participate, but in ways that are comfortable to them. Children do not enjoy mat time as much as teachers think they do. One reason for this was the activity provided and the opportunities given for individual children to participate. Study One analyses associated children's participation with their attentiveness and enjoyment. However, some children might possess effective strategies to be chosen, such as obtaining a highly visible sitting position or having friends help them. Some children require assistance to participate, although cold calling children is not recommended. Instead, it is recommended that teachers utilise children 's interests in specific activity as an incentive to participate. This may be more effective than using children's interest in content. A successful example given in a previous study included response cards (Wood et al., 2009). Small group work is another example.

Children were given the opportunity to work in small groups on the mat. However, what was evident was that when children are able to self-select the members of their group, certain children are likely to be left out. Previous studies have also suggested that academic-homophily might occur, where like-students group together. When teachers select groups, they are better able to prevent exclusion and can bring diverse children together who might not ordinarily interact. This potentially has positive benefits for the social climate of the classroom. 
Certain children's disruptive behaviours were seen as a big issue by children and teachers alike. Few teachers indicated that mat time was heavily characterised by rules; however, it was a strong theme in the children's interviews. Many of the rules pertained to children's bodies, such as how they must sit. At least some of the children's disruptive behaviour might arise from children attempting to reclaim agency over their bodies, or as a general bid to challenge power in playful ways. Whatever the reason, teachers' reactions to disruptive behaviour is viewed by the rest of the class. Some of the children indicated that they had negatively judged peers, and referred to certain children as "naughty" or "lazy". For some children this could have a negative influence on their reputation within the peer group, as well as their self-concept.

In fact, feedback matters a great deal given that some of the children made social comparisons between their performance on the mat and that of their peers. Some children gave particularly derogatory judgments of peers, whereas other children were fearful of attracting negative judgments. In cases such as these, it is unlikely that mat time enhances self-esteem, as many teachers reported. A second consideration is how competence is perceived in the classroom. There may be a responsibility for teachers to find and make visible children's various ways of knowing and doing. This means a culture whereby it is not merely knowledge of information that is emphasised, but that cultural knowledge and interests are valued as well as prosocial traits such as empathy, enabling opportunities for others and collaboration. Overall, in the present research, it seemed that domain knowledge and information prioritised by the NZCF (Ministry of Education, 2007) were highly valued at mat time, and this emphasis reinforced specific individualistic and egoistic traits in some children.

The findings of the research suggest that teachers should review the mat time climate, and consider whether their pedagogical goals are being met. Furthermore, experimentation with different seating and grouping configurations would enable a variety of social interactions, and could contribute in positive 
ways to the mat time climate. Other aspects that teachers could question include how children's mastery is prioritised against their performance, what types of questions are issued to children, and the role that the questioning plays in children's social worlds. It is recommended that teachers ensure that children's opportunities for participation are distributed by using collaborative activities that children are interested in. Furthermore, when the class is broken up into small groups, the teachers should select group membership rather than allow children to self-select.

In summary, how participating happens within the interactional milieu, has the potential to influence social and academic outcomes for individual children in either positive or negative ways. The interactional milieu is characterised by aspects of the official space of teacher authority and pedagogy. The children's social worlds intersect with this official space; children's peer cultures, dominance hierarchies, and friendships each impact on how individuals operate within the interactional milieu. Some children's learning or reputation might be enhanced by whether or not they can bring their questions, interests, and ideas to teachers' and peers' notice; this appears to require strategic knowledge of the milieu and the support of others. Conversely, others will struggle because of actual or perceived lack of social endorsement or assistance. In other words, children's repertoires for participating in the interactional milieu of mat time, and the fairness of the milieu dynamics, are important pedagogical considerations. This is especially so, given that a learning environment is known to influence, not only an individual's activity and learning, but their self-concept as well (Skinner et al., 1990). 


\section{REFERENCES}

Abd-Kadir, J., \& Hardman, F. (2007). The discourse of whole class teaching: A comparative study of Kenyan and Nigerian primary English lessons. Language and Education, 21(1), 1-15. doi:10.2167/le684.0

Adams, P. (2006). Exploring social constructivism: theories and practicalities. Education 3-13: International Journal of Primary, Elementary and Early Years Education, 34(3), 243-257, doi: 10.1080/03004270600898893

Adams, R. (1969). Location as a feature of instructional interaction. Merrill-Palmer Quarterly, 15, 309-321.

Adler, P., \& Adler, P. (1995). Dynamics of inclusion and exclusion in preadolescent cliques. Social Psychology Quarterly, 58(3), 145-154. doi.org/10.2307/2787039

Ainley, M., Hidi, S., \& Berndorff, D. (2002). Interest, learning, and the psychological processes that mediate their relationship. Journal of Educational Psychology, 94(3), 545-561. doi:10.1037//0022-0663.94.3.545

Alsaif, F. (2011). Intelligence-friendly environments (Master's thesis). School of Architecture, Victoria University of Wellington, New Zealand.

Alexander, J. M., Johnson, K. E., Leibham, M. E., \& Kelley, K. (2008). The development of conceptual interests in young children. Cognitive Development, 23, 324-334.

Alton-Lee, A. (2003). Quality teaching for diverse students in schooling: Best evidence synthesis. Wellington, New Zealand: Ministry of Education.

Anderson, G. (1999). Fundamentals of educational research (2nd ed.). London, UK: Falmer Press.

Andrews, J., \& Yee, W. (2006). Children's funds of knowledge and their real life activities: Two minority ethnic children learning in out-of-school contexts in the UK. Educational Review, 58(4), 435-449.

doi.org/10.1080/00131910600971909

Arthur, L., Beecher, B., Death, E., Farmer, S., \& Dockett, S. (2014). Programming and planning in early childhood settings. Melbourne, Victoria: Cengage. 
Asher, S. \& McDonald, K. (2010). Children's friendships. In B. McGraw, P Peterson, \& E. Baker (Eds.), International encyclopedia of education ( $3^{\text {rd }}$ ed., pp. 564569). Oxford, UK: Elsevier.

Avgitidou, S. (2001). Peer culture and friendship relationships as contexts for the development of young children's pro-social behaviour. International Journal of Early Years Education, 9(2), 145-152. doi:10.1080/713670688

Barton, E., Reichow, B., Woldery, M., \& Chen, C. (2011). We can all participate! Adapting circle time for children with autism. Young Exceptional Children, 14(2) 2-21. doi.org/10.1177/1096250610393681

Baum, S., Olenchak, R., \& Owen, S. (1998). Gifted students with attention deficits: Fact or fiction? Or, can we see the forest for the trees? Gifted Child Quarterly, 42(2), 96-104. doi: 10.1177/001698629804200204

Benenson, J., \& Heath, A. (2006). Boys withdraw more in one-on-one interactions, whereas girls withdraw more in groups. Developmental Psychology, 42(2), 272-282. doi.org/10.1037/0012-1649.42.2.272

Björk-Willén, P. (2008). Routine trouble: How preschool children participate in multilingual instruction. Applied Linguistics, 29(4), 555-577. doi:10.1093/applin/amm051

Bohning, G. (1981). Show and tell: Assessing oral language abilities. Reading Horizons, 22(1), 43-48.

Brown, J. (2006). Interviewer as instrument: Accounting for human factors in evaluative research. Evaluative Review, 30(2), 188-208. doi:

10.1177/0193841X05277222

Brown, D., \& Thomson, C. (2000). Cooperative learning in New Zealand schools. Wellington, New Zealand: Dunmore Press.

Bruhn, J. (2009). The group effect: Social cohesion and health outcomes. London, UK: Springer. doi: 10.10007/978-1-4419-0364-8

Buhs, E., Ladd, G., \& Herald, S. (2006). Peer exclusion and victimization: Processes that mediate the relation between peer group rejection and children's classroom engagement. Journal of Educational Psychology, 98(1), 1-13. doi:10.1037/0022-0663.98.1.1 
Bukowski, W., Buhrmester, D., \& Underwood, M. (2011). Peer relations as a developmental context. In M. Underwood \& L. Rosen (Eds.), Social development: Relationships in infancy, childhood, and adolescence (pp. 153179). New York, NY: Guildford.

Bukowski, W., Hoza, B., \& Boivin, M. (1994). Measuring friendship quality during pre- and early adolescence: The development and psychometric properties of the Friendship Qualities Scale. Journal of Social and Personal Relationships, 11, 471-484. doi.org/10.1177/0265407594113011

Buhrmester, D. (1996). Need fulfillment, interpersonal competence and the developmental contexts of adolescent friendship. In W. Bukowski, A. Newcomb, \& W. Hartup (Eds.). The company they keep in childhood and adolescence (pp. 158-185). New York, NY: Cambridge University Press.

Canney, C., \& Byrne, A. (2006). Evaluating circle time as a support to social skills development: Reflections on a journey in school-based research. British Journal of Special Education, 33(1), 19-24. doi.org/10.1111/j.14678578.2006.00407.x

Carr, M. (2001). Assessment in early childhood settings. London, England: Paul Chapman.

Cazden, C. (2001). Classroom discourse: The language of teaching and learning. Portsmouth, NH: Heinemann.

Cefai, C., Ferrario, E., Cavioni, V., Carter, A., \& Grech, T. (2014). Circle time for social and emotional learning in primary school. Pastoral Care and Education: An International Journal of Personal, Social and Emotional Development, 32(2), 116-130. doi:10.1080/02643944.2013.861506

Charlesworth, W., \& La Freniere, P. (1983). Dominance, friendship, and resource utilization in preschool children's groups. Ethology and Sociobiology, 4(3), 175-186. doi:10.1016/0162-3095(83)90028-6

Chen, A., Darst, P., \& Pangrazi. R. (2001). An examination of situational interest in its sources in physical education. British Journal of Educational Psychology, 71(3), 383-400. doi.org/10.1348/000709901158578 
Chen, Q., Hughes, J., Liew, J. \& Kwok, O. (2010). Joint contributions of peer acceptance and peer academic reputation to achievement in academically atrisk children: Mediating processes. Journal of Applied Developmental Psychology, 31(6), 448-459. doi: 10.1016/j.appdev.2010.09.001

Chen, W. \& Looi, C. (2011). Active classroom participation in a group scribbles primary science classroom. British Journal of Educational Technology, 42(4), 676-686. doi: 10.1111/j.1467-8535.2010.01082.x

Churchill, S. (2003). Goodness of fit in early childhood settings. Early Education Journal, 31(2), 113-118. doi.org/10.1023/B:ECEJ.0000005310.82353.bf Cillessen, A., Jiang, X., West, T., \& Laszkowski, D. (2005). Predictors of dyadic friendship quality. International Journal of Behavioural Development, 29, 165-172. doi:10.1080/01650250444000360

Clark, J. (2010). National Standards: The public debate- What was it all about? New Zealand Journal of Teachers' Work, 7(2), 106-124.

Coles, J. (2005). Strategic voices? Problems in developing oracy through interactive whole-class teaching. Changing English: Studies in Culture and Education, 12(1), 113-123. doi:10.1080/1358684052000340506

Collins, B. (2013). Empowerment of children through circle time: Myth or reality? Irish Educational Studies, 32(4), 421-436. doi:10.1080/03323315.2013.855559

Cook, C., Collins, T., Dart, E., Vance, M., McIntosh, K., Grady, E., \& Decano, P. (2014). Evaluation of the class pass intervention for typically developing students with hypothesised escape motivated classroom behaviour. Psychology in the Schools, 51(2), 107-125. doi:10.1002/pits.21742

Corsaro, W. (1985). Friendship and peer culture in the early years. Norwood, NJ: Ablex.

Corsaro, W. (1988). Peer culture in the preschool. Theory into Practice, 27(1), 1924. doi.org/10.1080/00405848809543326

Corsaro, W. (2003). We're friends, right? Washington DC: Joseph Henry Press. Corsaro, W. (2012). Interpretive reproduction in children's play. American Journal of Play, 4(4), 488-517. 
Corsaro, W. \& Molinari, L. (2008). Entering and observing children's worlds. In P. Monrad Christensen \& A. James (Eds.), Research with children: Perspectives and practices (pp. 239-290). New York, NY: Routledge.

Corsaro, W., \& Rizzo, T. (1988). Discussion and friendship: Socialisation process in the peer culture of Italian nursery school children. American Sociological Review, 53(6), 879-894. doi.org/10.2307/2095897

Creswell, J. (1994). Research design: Qualitative and quantitative approaches. Thousand Oaks, CA: Sage Publications.

Crotty, M. (1998). The foundations of social research: Meaning and perspective in the research process. London: Sage.

Cullen, J. (1991). Young children's learning strategies. International Journal of Early Childhood, 23(1), 44-58.

Danielewicz, J., Rogers, D., \& Noblit, G. (1996). Children's discourse patterns and power relations in teacher-led and child-led sharing time. Qualitative Studies in Education, 9(3), 311-331. doi.org/10.1080/0951839960090306

De Haan, D. \& Singer, E. (2001). Young children's language of togetherness. International Journal of Early Years Education, 9(2), 118-124. doi:10.1080/713670682

DeMars, C. (2010). Item response theory: Understanding statistics measurement. New York, NY: Oxford University Press.

Dennisen, J., Zarrett, N., \& Eccles, J. (2007). I like to do it, I'm able, and I know I am: Longitudinal couplings between domain-specific achievement, selfconcept, and interest. Child Development, 78(2), 430-447. doi: 10.111/j.1467-8624.2007.01007.x

Denscombe, M. (2007). The good research guide: For small-scale research projects. Maidenhead, United Kingdom: Open University Press.

DeRosier, M., Cillessen, A., Coie, J., \& Dodge, K. (1994). Group social context and children's aggressive behaviour. Child Development, 65, 1968-1079. doi: 10.1111/j.1467-8624.1994.tb00803.x

DiCarlo, C., Pierce, S., Baumgartner, J., Harris, M., \& Ota, C. (2012). Whole-group instruction practices and children's attention: A preliminary report. Journal 
of Research in Childhood Education, 26(2), 154-168.

doi:10.1080/02568543.2012.657744

Dijkstra, J., Lindenberg, S., \& Veenstra, R. (2008). Beyond the classroom norm: The influence of bullying popular adolescents and its relation to peer acceptance and rejection. Journal of Abnormal Child Psychology, 36, 12891299. doi: $10.1007 /$ s10802-008-9251-7

Dijkstra, J., Kretschmer, T., Lindenberg, S., \& Veenstra, R. (2015) Hedonic, instrumental, and normative motives: Differentiating patterns for popular, accepted, and rejected adolescents. Journal of Early Adolescence, 35(3), 308-328. doi: 10.1177/0272431614535092

Dijkstra, J., Cillessen, A., Lindenberg, S., \& Veenstra, R. (2015). Basking in reflected glory and its limits: Why adolescents hang out with popular peers. Journal of Research on Adolescence, 20, 942-958. doi.org/10.1111/j.15327795.2010.00671.x

Dion, K. (2000). Group cohesion: from field of forces to multidimensional construct. Group Dynamics: Theory, Research and Practice, 4(1), 7-26. doi: 10.1037/1089-2699.4.1.7

Donohue, K., Perry, K., \& Weinstein, R. (2003). Teachers' classroom practices and children's rejection by their peers. Applied Developmental Psychology, 24, 91118. doi: 10.1016/S0193-3973(03)00026-1

Droege, K. \& Stipek, D. (1993). Children's use of dispositions to predict classmates' behaviour. Developmental Psychology, 29, 646-654. doi: 10.1037/00121649.29.4.646

Duchesne, S., McMaugh, A., Bochner, S., \& Krause, K-L. (2013). Educational psychology for learning and teaching (4th ed.). Melbourne, Victoria: Cengage.

Duman, G. (2009). Implementing circle time activities in a Turkish national preschool. Procedia Social and Behavioural Sciences, 1, 1730-1733. doi.org/10.1016/j.sbspro.2009.01.306 
Edelman, M., \& Omark, D. (1973). Dominance hierarchies in young children. Social Science Information, 12(1), 103-110. doi.org/10.1177/053901847301200105

Eggen, P., \& Kauchak, D. (2006). Strategies and models for teachers: Teaching content and thinking skills ( $5^{\text {th }}$ ed.). Boston, MA: Pearson.

Eggen, T. \& Verhelst, N. (2011). Item calibration in incomplete testing designs. Psicológica, 32, 107-132.

Eirich, J. (2006). Classroom meeting: A window into children's cultures (PhD diss.). Graduate School of the Ohio State University, Ohio.

El-Ghoroury, N. (2002). The effects of reputation and teacher feedback on children's recall and perception of peers (PhD diss.). Graduate School of Binghamton University, NY.

Emilson, A. (2007). Young children's influence in preschool. International Journal of Early Childhood, 39(1), 11-38. doi.org/10.1007/BF03165946

Ernst, G. (1994). Talking circle: Conversation and negotiation in the ESL classroom. TESOL Quarterly, 28(2), 293-322. doi.org/10.2307/3587435

Fallace, T. (2015). The savage origins of child-centred pedagogy, 1871-1913. American Educational Research Journal, 52(1), 73-103. doi:10.3102/002831214561629

Farley, J., Risko, E., \& Kingstone, A. (2013). Everyday attention and lecture retention: The effects of time, fidgeting and mind wandering. Frontiers of Psychology, 4. 1-9. doi:10.3389/fpsycg.2013.00619

Farmer, T., McUliffe, M., \& Hamm, J. (2011). Revealing the invisible hand: The role of teachers in children's peer experiences. Journal of Applied Developmental Psychology, 32, 247-256. doi:10.1016/j.appdev.2011.04.006

Fein, M. (2012). Human hierarchies: A general theory. Piscataway, NJ: Transaction. Fingerson, L. (2011). Children's bodies. In J. Qvortrup, W. Corsaro, \& A. Honig (Eds.), The Palgrave handbook of childhood studies (pp. 217-227). Hampshire, United Kingdom: Palgrave Macmillan. 
Finlinson, A., Austin, A., \& Pfister, R. (2000). Cooperative games and children's positive behaviours. Early Childhood Development and Care, 164, 29-40. doi.org/10.1080/0300443001640103

Firth, R. (1972). Verbal and bodily rituals of greeting and parting. In J. LaFontaine (Ed.), The interpretation of ritual (pp. 1-38). Abingdon, Oxfordshire: Routledge.

Foster, L., Krohn, K., McCleary, D., Aspiranti, K., Nalls, M., Quillivan, C., Taylor, C., \& Williams, R. (2009). Increasing low-responding students' participation in class discussion. Journal of Behaviour and Education, 18, 173-188. doi:10.1007/s10864-009-9083-8

Freeman, M. \& Mathison, S. (2009). Researching children's experiences. New York, NY: Guidford Press.

Frieberg, H., \& Driscoll, A. (1996). Universal teaching strategies (2 ${ }^{\text {nd }}$ ed.). Needingham Heights, MA: Allyn \& Bacon.

Frisby, B., Berger, E., Burchett, M., Herovic, E. \& Strawser, M. (2014). Participation apprehensive students: The influence of face support and instructorstudent rapport on classroom participation. Communication Education, 63(2), 105-123. doi: 10.1080/03634523.2014.881516

Galbraith, J. (2011). Welcome to our team, Shark-boy. In D. Fernie, S. Madrid \& R. Kantor (Eds.), Educating toddlers to teachers: Learning to see and influence peer cultures of classrooms (pp. 37-62). New York, NY: Hampton Press.

Gallas, K. (1992). When children take the chair: A study of sharing time in a primary classroom. Language Arts, 69, 172-182.

Galton, M., Hargreaves, L., \& Pell, T. (2009). Group work and whole-class teaching with 11 to 14-year-olds compared. Cambridge Journal of Education, 39(1), 119-140. doi:10.1080/03057640802701994

Garandeau, C., Lee, I., \& Salmivalli, C. (2014). Inequality matters: Classroom status hierarchy and adolescents' bullying. Journal of Youth and Adolescence, 43, 1123-1133. doi:10.1007/s10964-013-0040-4 
Garrett, T. (2008). Student-centred and teacher-centred classroom management: A case study of three elementary teachers. Journal of Classroom Interaction, 43(1), 34-47.

Geddis, N. (1996). Science teaching and reflection: Incorporating new subjectmatter into teachers' classroom frames. International Journal of Science Education, 18(2), 249-265. doi.org/10.1080/0950069960180208

Gest, S., Domitrovich, C., \& Welsh, J. (2005). Peer academic reputation in elementary school: Associations with changes in self-concept and academic skills. Journal of Educational Psychology, 97(3), 337-346. doi: 10.1037/0022-0663.97.3.337

Gibbs, C., \& Wilks, A. (1991). Mat time in kindergartens: Aims and objectives for programme and children's development. (Unpublished Research Report). Palmerston North, New Zealand: Massey University.

Good, T., \& Brophy, J. (2003). Looking into classrooms (9th ed.). Boston, MA: Pearson.

Green, V. A., \& Rechis, R. (2006). Children's cooperative and competitive interactions in a limited resource situation: A literature review. Applied Developmental Psychology, 27, 42-59. doi.org/10.1016/j.appdev.2005.12.002

Green, V., Cillessen, A., Rechis, R., Patterson, M., \& Hughes, J. (2008). Social problem solving and strategy use in young children. The Journal of Genetic Psychology, 169(1), 92-112. doi: 10.3200/GNTP.169.1.92-112

Greer, L. (2012). Group cohesion: Then and now. Group research, 43(6), 655-661. doi:10.1177/1046496412461532

Gutierrez, K., \& Stone, L. (2000). Synchronic and diachronic dimensions of social practice: An emerging methodology for cultural-historical perspectives on literacy learning. In C. Lee \& P. Smagorinsky (Eds.). Vygotskian perspectives on literacy research: Constructing meaning through collaborative inquiry (pp.150-164). New York, NY: Cambridge University Press.

Han, M., Roskos, K., Christie, J., Mandzuk, S., \& Vukelich, C. (2005). Learning words: Large group time as vocabulary development opportunity. Journal of 
Research in Childhood Education, 19(4), 333-345.

doi:10.1080/02568540509595075

Hargreaves, V. (2013). What are working theories? And what can we do to support them? Early Education, 54, 34-37.

Harmon-Jones, C. (2011). Does musical behaviour promote affiliation? (PhD

Thesis). A \& M University, Texas.

Hartup, W. (1996). The company they keep: Friendships and their developmental significance. Child Development, 67(1), 1-13. doi.org/10.2307/1131681

Hartup, W. (2000). The company they keep. In W. Craig (Ed.), Childhood social development (pp. 59-84). Malden, MA: Blackwell.

Hartup, W., Laursen, B., Stewart, M., \& Eastenson, A. (1988). Conflict and friendship relations of young children. Child Development, 59(6), 15901600. doi.org/10.2307/1130673

Hawley, P. (1999). The ontogenesis of social dominance: A strategy-based evolutionary perspective. Developmental Review, 19, 97-132. doi.org/10.1006/drev.1998.0470

Henn, M., Weinstein, M., \& Foard, N. (2009). A critical introduction to social research. London, England: Sage.

Hidi, S. \& Harackiewitz, J. (2000). Motivating the academically unmotivated: A critical issue for the $21^{\text {st }}$ century. Review of Educational Research, 70, 151179

Hidi, S., \& Renninger, K. (2006). The four-phase model of interest. Educational Psychologist, 41(2), 11-127. doi.org/10.1207/s15326985ep4102_4

Hobday-Kusch, J., \& McVittie, J. (2002). Just clowning around: Classroom perspectives on children's humour. Canadian Journal of Education, 27(2/3), 195-210. doi.org/10.2307/1602220

Hogg, L. (2013). Applying funds of knowledge in a New Zealand high school: The emergence of team-based collaboration as an approach (PhD thesis). Victoria University of Wellington, New Zealand.

Hong, Y. (1995). Teaching large-group time in a preschool classroom: The teacher as orchestra conductor (PhD thesis). Illinois University, Urbana, USA. 
Houser, M., \& Frymier, A. (2009). The role of student characteristics and teacher behaviours in students' learner empowerment. Communication Education, 58(1), 35-53. doi:10.1080/03634520802237383

Howes, C. (1983). Patterns of friendship. Child Development, 54, 1041-1053. doi.org/10.2307/1129908

Howes, C. (1990). Social status and friendship from kindergarten to third grade. Journal of Applied Developmental Psychology, 11, 321-330. doi.org/10.1016/0193-3973(90)90013-A

Hughes, J., \& Zhang, D. (2007). Effects of the structure of classmates' perceptions of peers' academic abilities on children's perceived cognitive competence, peer acceptance, and engagement. Contemporary Educational Psychology, 32(3), 400-419. doi.org/10.1016/j.cedpsych.2005.12.003

Hundert, J. (2007). Training classroom and resource preschool teachers to develop inclusive class interventions for children with disabilities. Journal of Positive Behaviour Interventions, 9(3), 159-173. doi.org/10.1177/10983007070090030401

Hynds, A. (2000). Airing our dirty laundry: A case study of collaborative behaviour within an action research project. (Master's thesis). School of Educational Psychology and Pedagogy, Victoria University of Wellington, New Zealand.

Ivankova, N., Creswell, J., \& Stick, S. (2006). Using mixed-methods sequential explanatory design: From theory to practice. Field Methods, 18(1), 3-20. doi:10.1177/1525822X05282260

Jenkins, P. (1999). Surveys and questionnaires. Wellington, NZ: New Zealand Council for Educational Research.

Jenkins, L., \& Kilpatrick Demaray, M. (2012). Social support and peer concept in relation to peer victimization and peer aggression. Journal of School Violence, 11(1), 56-74. doi:10.1080/15388220.2011.630958

Johnson, R. \& Onwuegbuzie, A. (2004). Mixed methods research: A research paradigm whose time has come. Educational Researcher, 33(7), 14-26. Johnston, P. (2004). Choice words: How our language affects children's learning. Portland, MA: Stenhouse. 
Johnston, J., Chater, M., \& Bell, D. (2002). Teaching the primary curriculum. Philadelphia, PA: Open University Press.

Jordan, E., Cowan, A., Roberts, J. (1995). Knowing the rules: Discursive strategies in young children's power struggles. Early Childhood Research Quarterly, 10, 339-358. doi.org/10.1016/0885-2006(95)90011-X

Joussemet, M., Koestner, R., Lekes, N., \& Houlfort, N. (2004). Introducing uninteresting tasks to children: A comparison of the effects of rewards and autonomy support. Journal of Personality, 72(1), 139-166. doi.org/10.1111/j.0022-3506.2004.00259.x

Kantor, R., Elgas, P., \& Fernie, D. (1989). First the look and then the sound: Creating conversations at circle time. Early Childhood Research Quarterly, 4, 433-448. doi.org/10.1016/0885-2006(89)90002-1

Kavanaugh, R., \& McCall, R. (1983). Social influencing among two-year-olds: The role of affiliative and antagonistic behaviours. Infant Behaviour and Development, 6, 39-52. doi.org/10.1016/S0163-6383(83)80006-X

Kawulich, B. (2005). Participant observation as a data collection method. Qualitative Social Research, 6(2), retrieved from http://nbnresolving.de/urn:nbn:de:0114-fqs0502430

Kelly, B. (1999). Circle time. Educational Psychology in Practice: Theory, Research and Practice in Educational Psychology, 15(1),40-44, doi:10.1080/0266736990150107

Kennedy, J., Kennedy, M. \& Rutherford-Johnson, T. (2013). The Oxford dictionary of music. Oxford, UK: Oxford University Press.

Kern, P., Wolery, M., \& Aldridge, D. (2007). Use of songs to promote independence in morning greeting routines for young children with autism. Journal of Autism and Developmental Disorders, 37(7), 1264-1271. doi:10.1007/s10803-006-0272-1

Kessler, S. (1989). Boys' and girls' effect on the kindergarten curriculum. Early Childhood Research Quarterly, 4(4), 479-503. doi: 10.1016/08852006(89)90005-7 
Killen, R. (2012). Effective teaching strategies: Lessons from research and practice $\left(6^{\text {th }}\right.$ ed). Melbourne, Victoria: Cengage.

Kindermann, T. A. (2007). Effects of naturally existing peer groups on changes in academic engagement in a cohort of sixth graders. Child Development, 78, 1186-1203. doi:10.1111/j.1467-8624.2007.01060.x

Klopper, C. (2008). Meeting the goals of Te Whāriki through music in the early curriculum. Australian Journal of Early Childhood, 33(1), 1-8.

Ladd, G. (1990). Having friends, keeping friends, making friends, and being liked by peers in the classroom: Predictors of children's early school adjustment? Child Development, 61, 1081-1100. doi.org/10.2307/1130877

Ladd, G., \& Coleman, C. (1997). Children's classroom peer relationships and early school attitudes: Concurrent and longitudinal associations. Early Education and Development, 8(1), 51-66. doi.org/10.1207/s15566935eed0801_5

Lambert, N., Stillman, T., Hicks, J., Kamble, S., Baumeister, R., \& Fincham, F. (2013). To belong is to matter: Sense of belonging enhances meaning in life. Personality and Social Psychology Bulletin, 39, 1418-1427. doi: $10.1177 / 0146167213499186$

Lang, P. (1998). Getting round to clarity: What do we mean by circle time? Pastoral Care in Education, 16(3), 3-10. doi:10.1111/1468-0122.00096

Leach, T., \& Lewis, E. (2012). Children's experiences during circle time: A call for research-informed debate. International Journal of Personal, Social, and Emotional Development, 31(1), 43-52. doi:10.1080/02643944.2012.702781

Liamputtong, P. (2007). Researching the vulnerable. London, UK: Sage Publications.

Ling, S., \& Barnett, D. (2013). Increasing preschool student engagement during group learning activities using a group contingency. Topics in Early Child Special Education, 33(3), 186-196. doi:10.1177/0271121413484595

Lopreato, J. (2006). Human sociology. Encyclopedia of sociology (4th ed., pp. 280288). New York: Macmillan. 
Lown, J. (2002). Circle time: The perception of teachers and pupils. Educational Psychology in Practice, 18(2), 93-102. doi:10.1080/02667360220144539 Mandel Morrow, L., \& Smith, J. (1990). The effects of group size on interactive storybook reading. Reading Research Quarterly. 25(3), 213-231. doi.org/10.2307/748003

Marsh, H., Köller, O., Trautwein, U., Lüdtke, O., \& Baumert, L. (2005). Academic self-concept, interest, grades, and standardized test scores: Reciprocal effects models of causal ordering. Child Development, 76, 397-416. doi:10.111/j/1467-8624.2005.00853.x

Mary, L. (2012). Fostering positive peer relations in the primary classroom through circle time and cooperative games. Education 3-13: International Journal of Primary, Elementary and Early Years Education, 42(2), 125-137. doi:10.1080/03004279.2012.662239

Marx, A., Fuhrer, U., \& Hartig, T. (1999). Effects of classroom seating arrangements on children's question-asking. Learning Environments Research, 2(3), 249263. doi: 10.1032/A:1009901922191

McClelland, D. (1961). The achieving society. Princeton, NJ: Van Nostrand.

McDonald, K., Wang, J., Menzer, M., Rubin, K., \& Booth-LaForce, C. (2011). Prosocial behaviour moderates the effects of aggression on young adolescents' friendships. International Journal of Developmental Science, 5, 127-137.

McGee, C., \& Fraser, D. (2012). The professional practice of teaching (4th ed.). Melbourne, Victoria: Cengage.

McGrew, W. (1972). An ethological study of children's behaviour. New York: Academic Press.

McLaren, P. (1999). Schooling as a ritual performance: Toward a political economy of educational symbols and gestures. Oxford, United Kingdom: Rowman \& Littlefield.

McPherson, M., Smith-Lovin, L., \& Cook, J. (2001). Birds of a feather: Homophily in social networks. Annual Review of Sociology, 27, 415-444. doi.org/10.1146/annurev.soc.27.1.415 
Mercer, S., \& DeRosier, M. (2010). Prospective investigation of teacher preference and children's perceptions of student-teacher relationship. Psychology in the Schools, 47(2), 184-192. doi:10.1002/pits.20463.

Merville, G. (1954). More than show and tell. Childhood Education, 31(1), 70.

Meyer, C., Klein, E., \& Genishi, C. (1994). Peer relationships among four preschool language learners in small group time. Early Childhood Research Quarterly, 9(1), 61-85. doi.org/10.1016/0885-2006(94)90029-9

Mikami, A., Swaim-Griggs, M., Reuland, M., \& Gregory, A. (2012). Teacher practices as predictors of children's social preference. Journal of School Psychology, 50, 95-111. doi:10.1016/j.jsp.2011.08.002

Miller, D., \& Moran, T. (2007). Theory and practice in self-esteem enhancement: Circle-time and efficacy-based approaches, a controlled evaluation. Teachers and Teaching: Theory and Practice, 13(6), 601-615. doi:10.1080/13540600701683549

Milman, N. (2009). Pay attention: How teachers and students construct attentiveness in First Grade classrooms (PhD diss.). University of California, Los Angeles.

Ministry of Education. (2004). Kei tua o te Pae. Wellington, New Zealand: Learning Media.

Ministry of Education (2005). Teacher Census, 2004. Wellington: Demographic and Statistical Analysis Unit, Data Management and Analysis Division. Retrieved from

http://www.educationcounts.govt.nz/publications/schooling/teacher_censu $S$

Ministry of Education. (2007). The NZ curriculum for English medium teaching and learning in years 1-13. Wellington, New Zealand: Learning Media.

Ministry of Education. (2015). Ministry funding deciles. Retrieved from http://parents.education.govt.nz/primary-school/schooling-in-nz/ministryfunding-deciles/ 
Mitchell, M. (1993). Situational interest: Its multifaceted structure in the secondary school mathematics classroom. Journal of Educational Psychology, 85, 424-436. doi: 10.1037/0022-0663.85.3.424

Mosley, J. (1998). Quality circle time in the primary classroom: Your essential guide to enhancing self-esteem, self-discipline and positive relationships. Wisbech, Cambridgeshire: Learning Development Aids.

Mosley, J. (2005). Circle time for young children. NY, New York: Routledge.

Moss, H., \& Wilson, V. (1998). Circle time: Improving social interaction in a year six classroom. Pastoral Care in Education: An International Journal of Personal, Social and Emotional Development, 16(3), 11-17. doi:10.1111/1468-0122.00097

Muijs, D., \& Reynolds, D. (2005). Effective teaching: Evidence and practice (2 ${ }^{\text {nd }}$ ed.). Thousand Oaks, CA: Sage.

Murphy, S. (2003). Sharing time goes awry...or does it? Journal of Research in Early Childhood Education, 17(2), 218-229. doi.org/10.1080/02568540309595011

Myhill, D. (2002). Bad boys and good girls? Patterns of interaction and response in whole-class teaching. British Educational Research Journal, 28(3), 339-352. doi.org/10.1080/01411920220137430

Myhill, D., Jones, S., \& Hopper, R. (2006). Talking, listening, learning: Effective talk in the primary classroom. Berkshire, England: Open University Press.

Nassaji, H., \& Wells, G. (2000). What is the use of 'triadic' dialogue? An investigation of teacher-student interaction. Applied Linguistics, 21(3), 376406. doi.org/10.1093/applin/21.3.376

Nelson, P., Kohnert, K., Sabur, S \& Shaw, D. (2005) Classroom noise and children learning through a second language: Double Jeopardy? Language, Speech and Hearing Services in Schools, 36(3), 219-229. doi: 10.1044/0161$1461(2005 / 022)$

Ojanen, T., Aunola, K., \& Salmivalli, C. (2007). Situation-specificity of children's social goals: Changing goals according to changing situations? The 
International Journal of Behavioural Development, 31(3), 232-241. doi:10.1177/0165025407074636

Olk, T. (2011). Children, generational relations and intergenerational justice. In J. Qvortrup, W. Corsaro, \& M. Honig (Eds.), The Palgrave handbook of childhood studies (pp. 188-201). Hampshire, United Kingdom: PalgraveMacmillan.

Osborne, J., Costello, A., \& Kellow, J. (2008). Best practices in exploratory factor analysis. In J. Osborne (Ed.), Best practices in quantitative methods, (pp. 86102). Thousand Oaks, CA: Sage Publications.

Papatheodorou, T. (2009). Exploring relational pedagogy. In T. Papatheodorou \& J. Moyles (Eds.), Learning together in the early years: Exploring relational pedagogy (pp. 3-17). Abingdon, United Kingdom: Routledge.

Pellegrini, A., Roseth, C., Mliner, S., Bohn, C., Van Ryzin, M., Vance, N., Cheatham, C., Tarullo, A. (2007). Social dominance in preschool classrooms. Journal of Comparative Psychology, 121(1), 54-64. doi:10.1037/0735-7036.121.1.54

Piters (1995). An investigation of a teacher's interaction patterns with students during mat time discussions (Dip.Ed. project). Wellington College of Education, New Zealand.

Poveda, D. (2001). La Ronda in a Spanish kindergarten classroom with a crosscultural comparison to sharing time in the USA. Anthropology and Education Quarterly, 32(3), 301-325. doi:10.1525/aeq.2001.32.3.301

Puriola, A-M. (2006). The multiple faces of everyday life. In R. Parker-Rees \& J. Willan (Eds.), Early years education, major themes in education (pp. 7697). Abington, United Kingdom: Routledge.

Putallaz, M., \& Sheppard, B. (1992). Conflict management and social competence. In C. Shantz \& W. W. Hartup (Eds.), Conflict in child and adolescent development. New York, NY: Cambridge University Press.

Raban, B. (2001). Talking to think, learn and teach. In P. Smith (Ed.), Talking classrooms: Shaping children's learning through oral language instruction (pp. 27-41). Newark, DE: International Reading Association. 
Raver, C. (2004). Placing emotional self-regulation in sociocultural and socioeconomic contexts. Child Development, 75(2), 346-353. doi.org/10.1111/j.1467-8624.2004.00676.x

Raver, S., Hester, P., Michalek, A., Cho, D., \& Anthony, N. (2013). Impact of an activity mini-schedule on the inattention of preschoolers with cochlear implants during a group activity. Education and Treatment of Children, 36(2), 15-32. Doi: 10.1353/etc.2013.0014

Renshaw, P., \& Asher, S. (1983). Children's goals and strategies for social interaction. Merrill-Palmer Quarterly, 29(3), 353-374.

Rogers, C., \& Frieberg, H. (1994). Freedom to learn (3 $3^{\text {rd }}$ ed.). Upper Saddle River, NJ: Prentice-Hall.

Rosen, L. \& Patterson, M. (2011). The self and identity. In M. Underwood \& L. Rosen (Eds.), Social development: relationships in infancy, childhood, and adolescence (pp. 73-100). New York, NY: Guildford.

Rothlein, L., Einspruch, E., \& Goldberg, S. (1988). A study of traditional circle games played in public school kindergartens across the United States. International Journal of Early Childhood, 20(2), 55-61.

Roy, R., \& Benenson, J. (2002). Sex and contextual effects on children's use of interference competition. Developmental Psychology, 38(2), 306-312. doi.org/10.1037/0012-1649.38.2.306

Rubenstein Reich, L. (1994). Circle time in preschool: An analysis of educational praxis. European Early Childhood Education Research Journal, 2(1), 51-59. doi:10.1080/13502939485207531

Ryan, R. \& Deci, E. (2000). Self-determination theory and the facilitation of intrinsic motivation, social development, and well-being. American Psychologist, 55(1), 68-78. doi: 10.1037/0003-066X.55.1.68

Sandberg, A., \& Eriksson, A. (2008). Children's participation in preschool - on the conditions of the adults? Early Childhood Development and Care, 180(5), 619631. doi:10.1080/03004430802181759 
Schraw, G., \& Lehman, S. (2001). Situational interest: A review of the literature and directions for future research. Educational Psychology Review, 13(1), 2352. doi.org/10.1023/A:1009004801455

Simpson, L., \& Oh, K. (2013). Using circle time books to increase participation in morning circle. Teaching Exceptional Children, 45(6), 30-36.

Sinclair Knight, L. (Ed.). (1995). Collins paperback French dictionary. Glasgow, Great Britain: Harper Collins.

Skinner, E., Wellborn, J., \& Connell, J. (1990). What it takes to do well in school and whether I've got it: A process model of perceived control and children's engagement and achievement in school. Journal of Educational Psychology, 82(1), 22-32. doi.org/10.1037/0022-0663.82.1.22

Stake, R. (1995). The art of case study research. Thousand Oaks, CA: Sage Publications.

Stemler, S. \& Tsai, J. (2008). Best practices in inter-rater reliability: Three common approaches. In J. Osborne. (Ed.), Best practices in Quantitative Methods (pp.29-49). Thousand Oaks, CA: Sage Publications.

Stephenson, J. (2008). Different traditions and practices. In P. Auberson \& S. Schuck (Eds.), Teacher learning and development (pp. 116-127). Dordrecht, Netherlands: Springer Science.

Stipek, D. (1991). Characterising early childhood education programmes. In L. Rescorla, M. Hyson, \& K. Hirsh-Pasek (Eds.), Academic instruction in early childhood: Challenge or pressure? New directions for child development, 53 (pp. 47-55). San Francisco, CA: Jossey Bass.

Strayer, F., \& Strayer, J. (1976). An ethological analysis of social agonism and dominance relations among preschool children. Child Development, 47, 980-989. doi.org/10.2307/1128434

Sugai, G. \& Horner, R. (2002). The evolution of discipline practices: School-wide behaviour supports. Child and Family Behaviour Therapy, 24(1/2), 23-50. Svinth, L. (2013). Children's collaborative encounters in preschool. Early Child Development and Care, 183(9), 1242-1257. doi:10.1080/03004430.2012.719228 
Tait-McCutcheon, S. (2014). Teacher practice in primary mathematics: A story of positioning (PhD thesis). Victoria University of Wellington, New Zealand.

Te Maro, P. (2010). Involving children and young persons who are Māori in research. Loveridge (Ed.). Involving children and young people in research in educational settings: A report to the Ministry of Education. (pp. 47-62), Wellington, NZ: Ministry of Education.

Te One, S. (2010). Involving children in research: Primary school. In J. Loveridge (Ed.). Involving children and young people in research in educational settings: A report to the Ministry of Education. (pp. 67-104), Wellington, NZ: Ministry of Education.

Thomas, A. \& Cress, S. (1977). Temperament and development. New York, NY: Brunner/Mazel.

Tominey, S., \& McClelland, M. (2011). Red light, purple light: Findings from a randomized trial using circle time games to improve behavioural self regulation in preschool. Early Education and Development, 22(3), 489-519. doi:10.1080/10409289.2011.574258

Treadwell, T., Reisch, E., Travaglini, L., \& Kumar, V. (2011). The effectiveness of collaborative story building and telling in facilitating group cohesion in a college classroom setting. International Journal of Group Psychotherapy, 61(4), 503-517. doi.org/10.1521/ijgp.2011.61.4.502

Valiente, C., Lemery-Chalfant, K., Swanson, J., \& Reiser, M. (2008). Prediction of children's academic competence from their effortful control, relationships, can classroom participation. Journal of Educational Psychology, 100(1), 6777. doi: 10/1037/0022-0663.100.1.67

Van Oers, B. \& Hännikäinen, M. (2001). Some thoughts about togetherness: An introduction. International Journal of Early Years education, 9(2), 101-108. doi: 10.1080/09669760120053466

Vargo, K. (2008). The effects of a multiple schedule on hand raising during circle time in preschool classrooms (Degree of Science diss.). Southern Illinois University at Carbondale, Ann Arbor, USA. 
Vaughn, B., Vollenweider, M., Bost, K., Azria-Evans, M. \& Snider, J. (2003). Negative interactions and social competence for preschool children in two samples. Merrill-Palmer Quarterly, 49, 245-279.

Vogt, W. \& Johnson, R. (2005). Dictionary of statistics and methodology: A nontechnical guide for the social sciences. Thousand Oaks, CA: Sage Publications.

Wallat, C., \& Green, J. (1979). Social rules and communicative contexts in kindergarten. Theory into Practice, 18(4), 275-284. doi.org/10.1080/00405847909542845

Watters, A. (2013). Recentering pedagogy in an age of narcissism. The Journal of General Education, 62(1), 52-66. doi: 10.1353/jge.2013.0006

Weaver, R., \& Qi, J. (2005). Classroom organization and participation: College students' perceptions. The Journal of Higher Education, 76(5), 570-601. doi:10.1353/jhe.2005.0038

Wells, G. (1993). Reevaluating the IRF sequence: A proposal for the articulation of theories of activities and discourse for the analysis of teaching and learning in the classroom. Linguistics and Education, 5(1), 1-37. doi:10.1016/S08985898(05)80001-4

Wentzel, K. (1993). Does being good make the grade? Journal of Educational Psychology, 85(2), 357-364. doi: 10.1037/0022-0663.85.2.357

Wentzel, K., \& Asher, S. (1995). The academic lives of neglected, rejected, popular, and controversial children. Child Development, 66(3), 754-763. doi.org/10.2307/1131948

Wentzel, K., \& Caldwell, L. (1997). Friendships, peer acceptance, and group membership: Relations to academic achievement in middle school. Child Development, 68(6), 1198-1209. doi.org/10.1111/j.14678624.1997.tb01994.x

White, M. (1999). Magic circles: Building self-esteem through circle time. Thousand Oaks, CA: Sage.

White, F., Hayes, B., \& Livesey, D. (2013). Developmental psychology from infancy to adulthood ( $3^{\text {rd }}$ ed.). Sydney, NSW: Pearson. 
White, K., Jones, J., \& Sherman, M. (1998). Reputation and information and teacher feedback: Their influences on children's perceptions of behaviour problem peers. Journal of Social and Clinical Psychology, 17(1), 11-37. doi.org/10.1521/jscp.1998.17.1.11

White, K., \& Kistner, J. (1992). The influence of teacher feedback on young children's peer preferences and perceptions. Developmental Psychology, 28(5), 933-940. doi.org/10.1037/0012-1649.28.5.933

Wiersma, W., \& Jurs, S. (2009). Research methods in education: An introduction (9 $9^{\text {th }}$ ed.). Boston, MA: Pearson.

Wigfield, A. \& Cambria, J. (2010). Students' achievement values, goal orientations, and interest: Definitions, development, and relations to achievement outcomes. Developmental Review, 30, 1-35. doi: 10.1016/j.dr.2009.12.001

Willson-Quayle, A. (2001). The effects of child-centred, teacher-directed, and scaffolded instruction on low-income, Latino preschoolers' task performance, motivation, and private speech ( $\mathrm{PhD}$ diss.). George Mason University, Fairfax, VA.

Wilson, E. (1975). Sociobiology: The new synthesis. Cambridge, MA: Harvard University Press.

Wood, C., Mabry, L., Kretlow, A., Ya-yu, L., \& Galloway, T. (2009). Effects of preprinted response cards on students' participation and off-task behaviour in a rural kindergarten classroom. Rural Special Education Quarterly, 28(2), 39-47.

Woodrow, K. (2006). Creative discursive wiggle room: The generation of third space in middle school urban science classrooms. A paper presented to the Annual Meeting of the American Educational Research Association, San Francisco.

Zaghlawan, H., \& Ostrosky, M. (2011). Circle time: An exploratory study of activities and challenging behaviour in Head Start classrooms. Early Childhood Educational Journal, 38, 439-448. doi: 10.1007/s10643-0100431-z 


\section{APPENDICES}

\section{Appendix A: Survey}

Tena Koe. This survey is about mat times. For the purposes of this survey, 'mat time' includes any time that the majority of the class is present on the mat. It includes times when the children are participating in a physical activity on the mat, as well as when they are seated on the mat. This survey contains 25 brief questions and typically takes just over five minutes to complete. Your responses are appreciated.

Q1. How many years have you been teaching?

$1-2$

3-5

$6-10$

$11-15$

$16-20$

More than 20

Q2. Are you female or male?

Female

Male

Q3.

How would you describe your school?

State primary

Private

Maori immersion

Affiliated to a religion

Q4.

Where is your school located?

Inner city

In a suburban area

Rural

Q5. What is your school's decile rating?

Q6.

How many mat times would your class have in a single day?

$1-3$

4-6

$7-9$

$10-12$

13 or more 
Q7. How long would your average mat time be?

Less than 10 minutes

10-20 minutes

21-30 minutes

$31-40$ minutes

41-50 minutes

longer than 50 minutes

Q8. How often do you use mat time for the following?

\begin{tabular}{|c|c|c|c|c|c|}
\hline & never & $\begin{array}{l}\text { only sometimes, } \\
\text { not every day }\end{array}$ & every day & $2-3$ times a day & $\begin{array}{c}3 \text { or more times } \\
\text { per day }\end{array}$ \\
\hline News & $\bigcirc$ & $\bigcirc$ & O & O & 0 \\
\hline Explaining a learning activity & $\bigcirc$ & $\bigcirc$ & $\bigcirc$ & $\bigcirc$ & $\bigcirc$ \\
\hline Reading a book & O & O & 0 & 0 & 0 \\
\hline Welcome & O & $\bigcirc$ & $\bigcirc$ & $\bigcirc$ & 0 \\
\hline Farewell & $\bigcirc$ & 0 & 0 & 0 & 0 \\
\hline $\begin{array}{l}\text { Teaching curriculum } \\
\text { knowledge }\end{array}$ & O & $\bigcirc$ & $\bigcirc$ & 0 & 0 \\
\hline $\begin{array}{l}\text { Discussion about social } \\
\text { issues within class }\end{array}$ & O & $\bigcirc$ & $\bigcirc$ & $\bigcirc$ & $\bigcirc$ \\
\hline Classroom management & O & O & O & O & O \\
\hline $\begin{array}{l}\text { Transitioning from one } \\
\text { activity to another }\end{array}$ & O & O & O & 0 & 0 \\
\hline Music, singing & 0 & $\bigcirc$ & $\bigcirc$ & 0 & 0 \\
\hline Games & $\bigcirc$ & $\bigcirc$ & $\bigcirc$ & $\bigcirc$ & $\bigcirc$ \\
\hline Discussion about rules & $\bigcirc$ & $\bigcirc$ & $\bigcirc$ & 0 & $\bigcirc$ \\
\hline $\begin{array}{l}\text { Acknowledging individual } \\
\text { children's good work or } \\
\text { behaviour }\end{array}$ & O & O & 0 & 0 & 0 \\
\hline
\end{tabular}

Q9. What else do you use mat time for? 
Q10. To what extent do you agree that mat time contributes to the following?

\begin{tabular}{|c|c|c|c|c|c|}
\hline & $\begin{array}{l}\text { strongly } \\
\text { disagree }\end{array}$ & Disagree & $\begin{array}{l}\text { Neither Agree } \\
\text { nor Disagree }\end{array}$ & Agree & Strongly Agree \\
\hline Fostering group cohesion & 0 & 0 & 0 & 0 & 0 \\
\hline $\begin{array}{l}\text { Enhancing children's self } \\
\text { esteem }\end{array}$ & 0 & 0 & 0 & 0 & 0 \\
\hline $\begin{array}{l}\text { Providing a sense of rhythm } \\
\text { for the child's day }\end{array}$ & 0 & 0 & 0 & 0 & 0 \\
\hline $\begin{array}{l}\text { Assisting children's } \\
\text { development of restraint and } \\
\text { self control }\end{array}$ & O & O & 0 & 0 & 0 \\
\hline $\begin{array}{l}\text { Development of children's } \\
\text { cognitive knowledge }\end{array}$ & 0 & 0 & 0 & 0 & 0 \\
\hline $\begin{array}{l}\text { Development of children's } \\
\text { language }\end{array}$ & O & 0 & 0 & 0 & 0 \\
\hline $\begin{array}{l}\text { Development of children's } \\
\text { listening skills }\end{array}$ & 0 & 0 & 0 & 0 & 0 \\
\hline $\begin{array}{l}\text { Development of children's } \\
\text { social understandings }\end{array}$ & 0 & 0 & 0 & 0 & 0 \\
\hline $\begin{array}{l}\text { Development of children's } \\
\text { motor skills }\end{array}$ & 0 & 0 & 0 & 0 & 0 \\
\hline
\end{tabular}

Q11. What are the other benefits for children?

Q12. What are the benefits for you as the teacher? 
Q13. How challenging do you find the following at mat time?

\begin{tabular}{|c|c|c|c|c|c|}
\hline & $\begin{array}{l}\text { Extremely } \\
\text { challenging }\end{array}$ & Challenging & $\begin{array}{l}\text { Sometimes } \\
\text { challenging and } \\
\text { sometimes easy }\end{array}$ & easy & Extremely easy \\
\hline $\begin{array}{l}\text { Balancing the needs of } \\
\text { individual children against } \\
\text { the needs of the group. }\end{array}$ & 0 & 0 & 0 & 0 & 0 \\
\hline $\begin{array}{l}\text { Delivering the curriculum } \\
\text { versus following children's } \\
\text { interests. }\end{array}$ & 0 & 0 & 0 & 0 & 0 \\
\hline $\begin{array}{l}\text { Managing non-compliant } \\
\text { behaviour. }\end{array}$ & 0 & 0 & 0 & 0 & 0 \\
\hline $\begin{array}{l}\text { Ensuring that all children who } \\
\text { want to have a turn to } \\
\text { contribute or participate. }\end{array}$ & 0 & 0 & 0 & 0 & 0 \\
\hline $\begin{array}{l}\text { Maintaining all children's } \\
\text { engagement. }\end{array}$ & 0 & 0 & 0 & 0 & 0 \\
\hline
\end{tabular}

Q14. Are there other aspects to mat time that are not listed here, and that you find challenging?

Q15. How do you engage children's attention at mat time? Please select as many as apply.

Focusing on what children are interested in

Giving verbal reprimands/ warnings

Offering rewards

Writing children's names on the board

Seating certain children close to the teacher

Removing disruptive children from the mat area

Using interesting voice intonation

Q16. What other strategies do you use to engage children's attention at mat time? 
Q17. To what extent do you agree with the following statements in relation to children's behaviour at mat time?

\begin{tabular}{l|ccccc} 
& $\begin{array}{l}\text { Strongly } \\
\text { disagree }\end{array}$ & Disagree & $\begin{array}{c}\text { Neither Agree } \\
\text { nor Disagree }\end{array}$ & Agree & Strongly Agree \\
\hline $\begin{array}{l}\text { Particular children tend to } \\
\text { dominate. }\end{array}$ & 0 & 0 & 0 & 0 \\
$\begin{array}{l}\text { Particular children observe } \\
\text { only. }\end{array}$ & 0 & 0 & 0 & 0 \\
All children are attentive. & 0 & 0 & 0 & 0 \\
$\begin{array}{l}\text { Particular children seem to } \\
\text { always put their hand up. }\end{array}$ & 0 & 0 & 0 & 0 \\
$\begin{array}{l}\text { Particular children are } \\
\text { consistently disruptive. }\end{array}$ & 0 & 0 & 0 & 0 \\
Children enjoy group time. & 0 & 0 & 0 & 0
\end{tabular}

Q18. Have you any comment that you would like to make about children's behaviour at mat time?

Q19. How likely is it that every child contributes or participates at mat time? Very Unlikely Unlikely Undecided Likely Very Likely

Q20. Have you any comments about children's contribution or participation at mat time?

Thank you for your responses so far. You are nearly at the end of the survey. 
Q21. Where would you place your mat time practices on these scales?

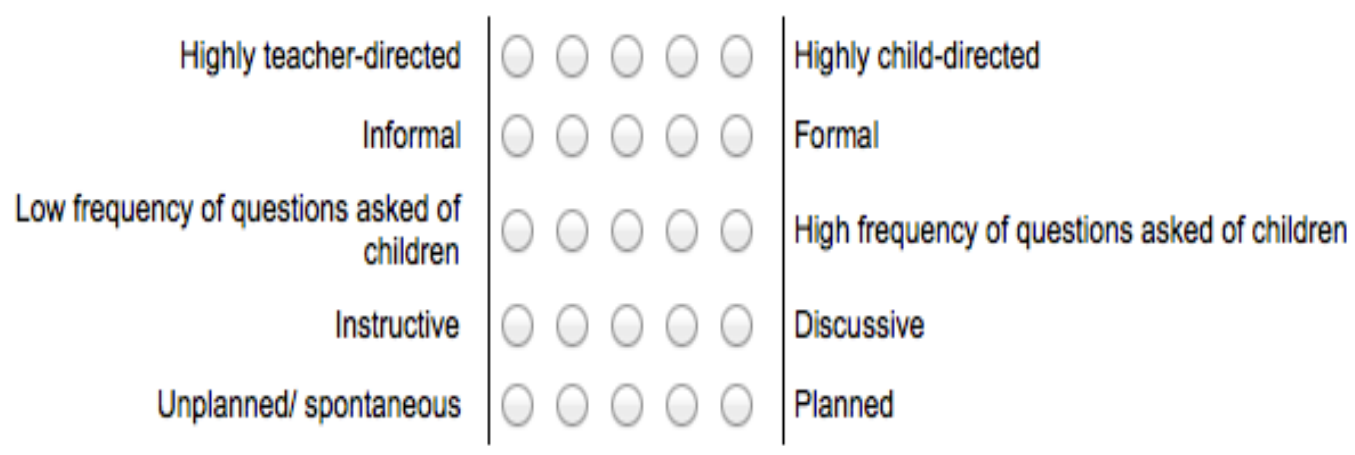

Q22. Have you any comments that you would like to make about your teaching strategies at mat time?

Q23. Have you any other comment that you would like to make about mat times?

Thank you for completing the survey. This is the final section. To ensure that your survey answers remain anonymous, any contact information given in regards to the following questions will be kept separate from your survey responses.

Q24. If you would like to receive a brief report outlining the findings of this survey, please provide your email address below.

Q25. Would you be interested in participating in further research about mat time? If so, please give your details below.

Q26. To thank you for your time, there is opportunity for your school to enter a draw for five prizes worth $\$ 200$ each. If your school is drawn, the money will be placed into your school's bank account. If you are interested in having your school included in the draw, please fill in the details below. School's name

School's email address 


\section{Appendix B: Ethics approval letter}

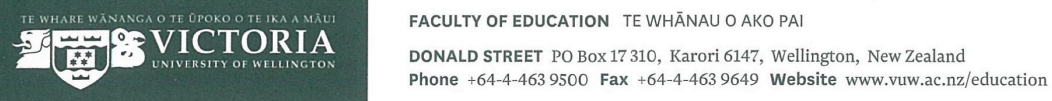

15 February 2011

Anita Mortlock

Lecturer

Victoria University of Wellington Faculty of Education

$\mathrm{C} /$ - School of Educational Psychology and Pedagogy

Donald Street

Wellington

Dear Anita

\section{RE: Ethics application SEPP/2010/115: RM 18188}

I am pleased to advise you that your ethics application 'Mat time in Primary school. How Teachers view it and how children experience it.' with requested amendments, has been approved by the Victoria University of Wellington Faculty of Education Ethics Committee. Please note that the approval for your research to commence is from the date of this letter.

Once you have translated all the information sheets and consent forms please provide electronic copies that can be filed with the other documentation from your application.

Best wishes for your research.

Yours Sincerely

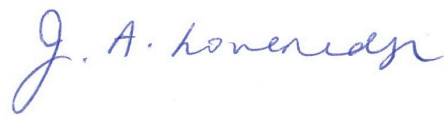

Dr Judith Loveridge

Co-Convener

Victoria University of Wellington Faculty of Education Ethics Committee 


\section{Appendix C: Information Letters}

\section{Information Letter to Principals}

Dear Principal,

Kia ora. I would like to invite you to participate in my Doctoral research project. The research proposal has been assessed by two reviewers, a panel of peers and the Victoria University of Wellington Faculty of Education Ethics Committee (Reference number: SEPP/2010/115:RM18188). This research will be supervised by Associate Professor Vanessa Green (School of Educational Psychology and Pedagogy)and Dr. Mary-Jane Shuker (School of Educational Policy and Implementation). The aim of the study is to gather teachers' and children's views about what mat time is for and how it is used, with a special focus on interactions that occur. Three specific questions will be addressed:

1. How do primary school teachers mat time?

2. What are teachers' perceptions of children's mat time experiences?

3. What are children's perceptions of their mat time experiences?

4. How are children's bids to communicate as an element of participation distributed across the group at mat time?

Year two classrooms have been chosen as the population for the study due to the comparative stability in class membership. The study has been designed to take place in three phases: the first will involve a large scale questionnaire; the second will involve interviews with children and teachers in three classrooms; and the final phase will consist of observations of group time in the three classrooms.

\section{Phase 1: Large scale questionnaire}

The first phase involved likert and multiple answer questions about the purpose of mat time, challenges for teachers and participation of children. The web tool Qualtics was used that does not identify participants.

\section{Phase 2: Interviews with teachers and children} Written consent is sought from you, children and their families. During this time, I would like to spend some time in the classroom to acclimatise the class members to my presence and build familiarity.

Interviews with Teachers

Once consent has been gained I would like to commence semi-structured interviews with you. This would take approximately an hour and would seek further detailed information about the purpose of mat time and the interactions that take place within it. These would take place during non-contact time or outside of school hours.

Interviews with Children

As part of this study, it is important that I interview as many children within the class as possible. Consent will be sought from the families of every child in the class and interviewswill take place with each child form who consent has been given. I recognise that it is important that the children's learning is not disrupted therefore you will be asked to choose when each child can participate in interviews. For example, you might choose a particular child because he or she had already finished one task and was not yet ready to commence the next. 
Children would have already given their written consent however in addition the child's verbal consent would be sought prior to being interviewed. Interviews would take place at a point nominated by the school. This will be away from the classroom but on the premises of the school, for example a resource room or staff room. Children would be provided with props associated with mat time and will be asked to set up the mat. In addition they would be asked to describe what happens at mat time and what they would like to have happen. Interviews with children would take no longer than 10 minutes and the researcher will return the child to his or her classroom. If a child appears to be uncomfortable during the interview process the interview will cease immediately and the researcher will accompany the child back to his or her classroom.

Permission will be sought from yourself and child participants to audiotape interviews. These will be transcribed. For your interview, transcripts and inferences will be made from it and will be returned for further comment and/or validation.

Phase three: classroom observations

I would like to return to the classroom for phase three which would involve filming mat time for no more than 10 minutes at a time over four days. The video footage would then be used to develop frequency counts of the different strategies that children in use in their attempts to communicate to the wider class.

\section{Timeline}

Term 1, 2011- Questionnaire sent to schools

Term 2, 2011- Interviews of children and teachers

Term 3, 2011- Observations

\section{Use of video}

I am aware that the use of video cameras in research with young children raises specific issues. As mentioned before, consent will be sought from principals, teachers, children and their families before filming commences. One issue that arises will be filming children for whom consent has not been granted. It is necessary that such children are not separated from the class at mat time, therefore it is likely that they will be filmed. No data will be taken of these children and the images of non-participant children will be blurred. All video footage will be kept in a secure environment and will be destroyed five years after the study has been completed.

\section{Confidentiality}

All information gathered as part of this study would remain confidential to myself, my two $\mathrm{PhD}$ supervisors and a colleague who will be asked to check some of the data as a reliability measure. The colleague will be asked to sign a confidentiality contract. The findings of this study will be presented in written and verbal reports, however, no names or identifying information about individuals or schools will be given at any point. Individual children's views about group time that emerge from the interviews will not be disclosed to their classroom teacher or any other party.

\section{Ethics}

The ethical application has been approved by the VUW Faculty of Education Human Ethics Committee (Reference number: SEPP/2010/115:RM18188). If at any time you have questions 
or concerns about this study, contact Dr. Allison Kirkman, Chair of the Victoria of University Human Ethics Committee (telephone +64 4463 5675, E-mail: Allison.kirkman@vuw.ac.nz).

Please note that there is no obligation for you to give consent to allow this study to take place in your primary school. Your decision about whether this study can go ahead will not affect your relationship with Victoria University of Wellington. If you choose to give consent you have the right to withdraw this at any time. Your decision to discontinue the study would not affect your relationship with Victoria University.

\section{Data storage and deletion}

All paper-based and video data will be kept in a locked filing cabinet in a locked office in the Faculty of Education at Victoria University. The data may be required in the process of PhD examination peer challenge and as such will be kept for five years after publication. After this time it will be destroyed through being shredded in the case of paper-based data (transcripts, printed off questionnaires), or wiped in the case of video data and audio tapes.

\section{Reporting/ Dissemination}

The findings of this study will be submitted to academic journals and/or professional journals for publication. Findings may also be presented at a conference. In either case, such reports will be given to you.

Thank you for considering this application to undertake research in your primary school. If you have any questions at any time, please feel free to contact me using the information below.

Sincerely,

Anita Mortlock

School of Educational Psychology and Pedagogy

Victoria University of Wellington

PO Box 17-310, Karori

Wellington, New Zealand

044639544

Anita.mortlock@vuw.ac.nz

PhD Supervisors

Associate Professor Vanessa Green

School of Educational Psychology and

Pedagogy

Victoria University of Wellington

PO Box 17-310, Karori

Wellington, New Zealand

044639574

vanessa.green@vuw.ac.nz
Dr. Mary Jane Shuker

School of Educational Policy and

Implementation

Victoria University of Wellington

PO Box 17-310, Karori

Wellington, New Zealand

044639659

mary-jane.shuker@vuw.ac.nz 


\section{Information Letter to Teachers}

Dear (Insert teacher's name),

Kia ora. I would like to invite you to participate in my Doctoral research project. The research proposal has been assessed by two reviewers, a panel of peers and the Victoria University of Wellington Faculty of Education Ethics Committee (Reference number: SEPP/2010/115/115:RM18188). This research will be supervised by Associate Professor Vanessa Green (School of Educational Psychology and Pedagogy)and Dr. Mary-Jane Shuker (School of Educational Policy and Implementation). The aim of the study is to gather teachers' and children's views about what mat time is for and how it is used, with a special focus on interactions that occur. Three specific questions will be addressed:

1. How do primary school teachers mat time?

2. What are teachers' perceptions of children's mat time experiences?

3. What are children's perceptions of their mat time experiences?

4. How are children's bids to communicate as an element of participation distributed across the group at mat time?

Year two classrooms have been chosen as the population for the study due to the comparative stability in class membership. The study has been designed to take place in three phases: the first will involve a large scale questionnaire; the second will involve interviews with children and teachers in three classrooms; and the final phase will consist of observations of group time in the three classrooms.

\section{Phase 1: Large scale questionnaire}

The first phase involved likert and multiple answer questions about the purpose of mat time, challenges for teachers and participation of children. The web tool Qualtics was used that does not identify participants.

\section{Phase 2: Interviews with teachers and children}

Written consent is sought from you, children and their families. During this time, I would like to spend some time in the classroom to acclimatise the class members to my presence and build familiarity.

Interviews with Teachers

Once consent has been gained I would like to commence semi-structured interviews with you. This would take approximately an hour and would seek further detailed information about the purpose of mat time and the interactions that take place within it. These would take place during non-contact time or outside of school hours.

Interviews with Children

As part of this study, it is important that I interview as many children within the class as possible. Consent will be sought from the families of every child in the class and interviewswill take place with each child form who consent has been given. I recognise that it is important that the children's learning is not disrupted therefore you will be asked to choose when each child can participate in interviews. For example, you might choose a particular child because he or she had already finished one task and was not yet ready to commence the next. Children would have already given their written consent however in addition the child's 
verbal consent would be sought prior to being interviewed. Interviews would take place at a point nominated by the school. This will be away from the classroom but on the premises of the school, for example a resource room or staff room. Children would be provided with props associated with mat time and will be asked to set up the mat. In addition they would be asked to describe what happens at mat time and what they would like to have happen. Interviews with children would take no longer than 10 minutes and the researcher will return the child to his or her classroom. If a child appears to be uncomfortable during the interview process the interview will cease immediately and the researcher will accompany the child back to his or her classroom.

Permission will be sought from yourself and child participants to audiotape interviews. These will be transcribed. For your interview, transcripts and inferences will be made from it and will be returned for further comment and/or validation.

Phase three: classroom observations

I would like to return to the classroom for phase three which would involve filming mat time for no more than 10 minutes at a time over four days. The video footage would then be used to develop frequency counts of the different strategies that children in use in their attempts to communicate to the wider class.

\section{Timeline}

Term 1, 2011- Questionnaire sent to schools

Term 2, 2011- Interviews of children and teachers

Term 3, 2011- Observations

\section{Use of video}

I am aware that the use of video cameras in research with young children raises specific issues. As mentioned before, consent will be sought from principals, teachers, children and their families before filming commences. One issue that arises will be filming children for whom consent has not been granted. It is necessary that such children are not separated from the class at mat time, therefore it is likely that they will be filmed. No data will be taken of these children and the images of non-participant children will be blurred. All video footage will be kept in a secure environment and will be destroyed five years after the study has been completed.

\section{Confidentiality}

All information gathered as part of this study would remain confidential to myself, my two $\mathrm{PhD}$ supervisors and a colleague who will be asked to check some of the data as a reliability measure. The colleague will be asked to sign a confidentiality contract. The findings of this study will be presented in written and verbal reports, however, no names or identifying information about individuals or schools will be given at any point. Individual children's views about group time that emerge from the interviews will not be disclosed to their classroom teacher or any other party.

\section{Ethics}

The ethical application has been approved by the VUW Faculty of Education Human Ethics Committee (Reference number: SEPP/2010/115:RM18188). If at any time you have questions 
or concerns about this study, contact Dr. Allison Kirkman, Chair of the Victoria of University Human Ethics Committee (telephone +64 4463 5675, E-mail: Allison.kirkman@vuw.ac.nz).

Please note that there is no obligation for you to give consent to allow this study to take place in your primary school. Your decision about whether this study can go ahead will not affect your relationship with Victoria University of Wellington. If you choose to give consent you have the right to withdraw this at any time. Your decision to discontinue the study would not affect your relationship with Victoria University.

\section{Data storage and deletion}

All paper-based and video data will be kept in a locked filing cabinet in a locked office in the Faculty of Education at Victoria University. The data may be required in the process of PhD examination peer challenge and as such will be kept for five years after publication. After this time it will be destroyed through being shredded in the case of paper-based data (transcripts, printed off questionnaires), or wiped in the case of video data and audio tapes.

\section{Reporting/ Dissemination}

The findings of this study will be submitted to academic journals and/or professional journals for publication. Findings may also be presented at a conference. In either case, such reports will be given to you.

Thank you for considering this application to undertake research in your primary school. If you have any questions at any time, please feel free to contact me using the information below.

Sincerely,

Anita Mortlock

School of Educational Psychology and Pedagogy

Victoria University of Wellington

PO Box 17-310, Karori

Wellington, New Zealand

044639544

Anita.mortlock@vuw.ac.nz

PhD Supervisors

Associate Professor Vanessa Green

Dr. Mary Jane Shuker

School of Educational Psychology and

School of Educational Policy and

Pedagogy

Victoria University of Wellington

PO Box 17-310, Karori

Implementation

Wellington, New Zealand

044639574

Victoria University of Wellington

PO Box 17-310, Karori

Wellington, New Zealand

vanessa.green@vuw.ac.nz

044639659

mary-jane.shuker@vuw.ac.nz 


\title{
Information Letter to Parents
}

\author{
Dear Parent/ guardian,
}

Kia ora. I would like to invite you to consider allowing your child to participate in my Doctoral research project. The research proposal has been assessed by two reviewers, a panel of peers and the Victoria University of Wellington Faculty of Education Ethics Committee (Reference number: SEPP/2010/115:RM18188). This research will be supervised by Associate Professor Vanessa Green (School of Educational Psychology and Pedagogy) and Dr. Mary-Jane Shuker (School of Educational Policy and Implementation). The aim of the study is to gather teachers' and children's views about what mat time is for and how it is used, with a special focus on interactions that occur. Three specific questions will be addressed:

1. How do primary school teachers mat time?

2. What are teachers' perceptions of children's mat time experiences?

3. What are children's perceptions of their mat time experiences?

4. How are children's bids to communicate as an element of participation distributed across the group at mat time?

\section{What will this study involve?}

If you agree to allow your child to participate, I would like to interview them about their thoughts about mat time. I recognise that it is important that the children's learning is not disrupted therefore each classroom teacher would be asked to choose when each child can participate in interviews. For example, a teacher might choose a particular child because he or she had already finished one task and was not yet ready to commence the next. Your child will be asked to give their written consent also, however in addition they will be asked if they feel okay about being interviewed at the time.

Interviews would take place at a point nominated by the school. This will be away from the classroom but on the premises of the school, for example a resource room or staff room. Children would be provided with props associated with mat time and will be asked to set up the mat. In addition they would be asked to describe what happens at mat time and what they would like to have happen. Interviews with children would take no longer than 10 minutes and the researcher will return the child to his or her classroom. If a child appears to be uncomfortable during the interview process the interview will cease immediately and the child will be returned to their classroom. After this, I would like to return to your child's classroom to film mat time for no more than 10 minutes at a time over four days. The video footage would then be used to gather information on the ways that children speak to the wider class.

\section{Use of video}

I am aware that the use of video cameras in research with young children raises specific issues. As mentioned before, consent will be sought from principals, teachers, children and their families before filming commences. One issue that arises will be filming children for whom consent has not been granted. It is necessary that such children are not separated from the class at mat time, therefore it is likely that they will be filmed. No data will be taken of these children and the images of non-participant children will be blurred. All video data will be securely stored and destroyed five years after the completion of the project. 


\section{Confidentiality}

All information gathered as part of this study would remain confidential to myself, my two $\mathrm{PhD}$ supervisors and a colleague who will be asked to check some of the data as a reliability measure. The colleague will be asked to sign a confidentiality contract. The findings of this study will be presented in written and verbal reports, however, no names or identifying information about individuals or schools will be given at any point. Individual children's views about group time that emerge from the interviews will not be disclosed to their classroom teacher or any other party.

\section{Ethics}

The ethical application has been approved by the VUW Faculty of Education Human Ethics Committee (Reference number: SEPP/2010/115:RM18188). If at any time you have questions or concerns about this study, contact Dr. Allison Kirkman, Chair of the Victoria of University Human Ethics Committee (telephone +64 4463 5675, E-mail: Allison.kirkman@vuw.ac.nz).

Please note that there is no obligation for you to give consent to allow this study to take place in your child's primary school. Your decision about whether this study can go ahead will not affect your relationship with Victoria University of Wellington. If you choose to give consent you have the right to withdraw this at any time. Your decision to discontinue the study would not affect your relationship with Victoria University.

\section{Data storage and deletion}

All paper-based and video data will be kept in a locked filing cabinet in a locked office in the Faculty of Education at Victoria University. The data may be required in the process of PhD examination and peer challenge. As such it will be kept for five years after publication. After this time it will be destroyed through being shredded in the case of paper-based data (transcripts, printed off questionnaires), or wiped in the case of video data and audio tapes.

\section{Reporting/ Dissemination}

The findings of this study will be submitted to academic journals and/or professional journals for publication. Findings may also be presented at a conference. In either case, copies of such reports will be given to the teacher and can also be obtained from me.

Thank you for considering this application to undertake research with your child. If you have any questions at any time, please feel free to contact me using the information below.

Sincerely, Anita Mortlock

School of Educational Psychology and Pedagogy

Victoria University of Wellington

PO Box 17-310, Karori

044639544

Anita.mortlock@vuw.ac.nz

PhD Supervisors:

Associate Professor Vanessa Green

Dr. Mary Jane Shuker

Victoria University of Wellington

044639574

Victoria University of Wellington

044639659

Vanessa.green@vuw.ac.nz

mary-jane.shuker@vuw.ac.nz 
Information Letter to children

Kia ora!

My name is Anita. I live in Wellington with my family and my pets. I am interested in mat time. I would like to find out about what you think of mat time. To do this, I would like to talk to you and ask you some questions.

I will tell you what will happen so that you can decide if you would like to talk to me about mat time. Your teacher will say that you can come with me to (insert place where interview will happen). I will show you a model of your classroom. You can help me set up the mat time. You can tell me what happens at mat time. You might also have ideas about what is fun to do at mat time that you would like to tell me.

I don't want to forget anything you tell me. I will record your voice on tape and I might do some writing. If you let me I will take a photograph of how we set up the mat. On some other days I will come and video record mat time in your classroom.

You don't have to take part. I will not be mad if you choose not to. This is a photo of me (insert photo). 


\section{Appendix D: Confidentiality agreement for transcription}

Confidentiality Agreement for Transcriber

Project: Mat time at school: How teachers view it and children experience it.

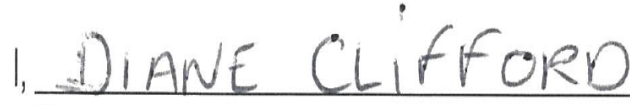

Will be assisting in this research project and as such, will be privy to some of the data.

No names of participants or any information leading to the identification of the schools will be given to me. All data that I view will be deemed confidential. I understand that I may not show the data to any third party, nor may I keep copies of it. Furthermore, I may not discuss the data with any third party.

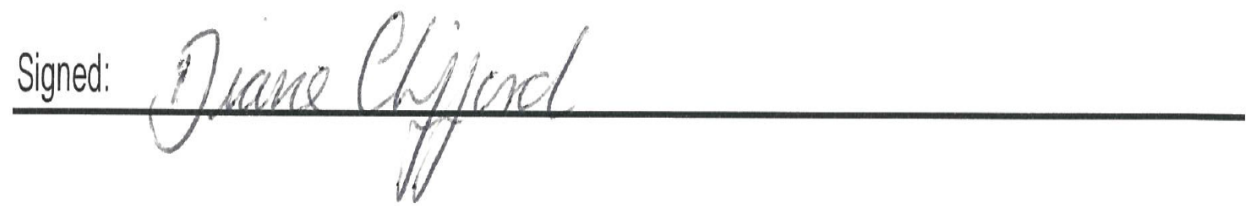

Date: $3 \cdot 5-12$ 


\section{Appendix E: Coding Instructions to establish inter-rather reliability}

\section{Coding}

The teachers are labeled AA1, BB2 and CC3.

Because the interviews were semi-structured, some of the responses take a circular or tangential route before they reach the research foci. For this reason, please read every interview in its entirety for each focus, as opposed to reading the responses directly underneath the relevant interviewer question. Where you are unsure of where to place something, please make a note of it. As you read through each interview, please place record the interview code against the relevant categories, e.g.

BEST PLACE TO SIT (please note- these have been fabricated as an example)

\begin{tabular}{|c|c|}
\hline \multirow[t]{7}{*}{ Front } & $\begin{array}{l}\text { See better } \\
\text { A12 }\end{array}$ \\
\hline & Hear Better \\
\hline & $\begin{array}{l}\text { Focus Better } \\
\text { A12, B4, B19 }\end{array}$ \\
\hline & Being noticed by the teacher \\
\hline & Confidence \\
\hline & Friends \\
\hline & $\begin{array}{l}\text { Other (please stipulate) } \\
\text { C6(sitting near giraffe picture) }\end{array}$ \\
\hline
\end{tabular}

\section{The best place to sit}

1. Where a child has indicated that the best place to sit is near one of the teachers, this is coded as 'the front'.

2. All interviewees coded with a $\mathrm{C}$ refer to a circle. There is a separate sheet for these.

3. Several children nominated more than one best place. In this instance, record each place that the child gives. In some instances, they nominate a best place for their friend. Please include this as well.

4. Working definitions for each place are as follows:

For interviews staring with an A or B:

Front: approximately the first two rows on the carpet.

Back: the last two rows of the group.

Middle: everything in between the front and the back.

Other: for example, rather than a set position a child might say that they sit where a friend is, or they sit where they can leave the mat more quickly. Please record the child's words when you place a response in this category. 
For Interviews staring with C (the children in this group are seated in a circle with the teacher):

Front: three positions either side of the teacher

Opposite: the six positions in the opposite side of the circle from the teacher

Sides: three to six spaces from the teacher's left of right

\section{Reasons for the best place to sit}

1. Several children nominated more then one reason for the best place to sit. Please code all of the reasons.

2. The reasons that children give need to be sorted into front, middle and back, e.g. so we know why children thought the front was the best place, etc. Definitions for reasons are as follows:

See better: the child refers to seeing the teacher or a resource (e.g. a book). Alternatively, they might just refer to being able to see better generally.

Hear better: the child might refer to other children being noisy as a reason to avoid sitting with them (example: I sit up the front because children are noisy at the back). They might also say that they can hear the teacher better.

Focus better: this refers to any references to concentration, focus, thinking clearly, following instructions more easily, staying on-task, avoiding distractions by peers, or being engaged in the learning activity.

Being noticed by the teacher: the child refers to any personal interaction with the teacher, as opposed to the teacher's interactions with the class.

Physical comfort: the nominated seating position is more physically comfortable, e.g. there may be a piece of equipment to lean against.

Confidence: The child feels greater emotional security when seated in their nominated best place. This includes because of proximity to the teacher or friends. Friends: They sit where their friends are.

\section{Importance of participation}

1. Not all children gave answers.

2. The focus is on sharing when the whole class is together. As such, please be mindful of when children refer to small group work and do not include it.

3. Definitions are as follows:

Important: the child believes that it is important that they share their ideas or participate in some way. They answered 'yes' when asked whether putting their hand up or sharing ideas were important.

Not important: does not believe any form of personal participation is important. The child answered 'no' when asked questions about whether it was important that they put their hand up or share ideas or answers.

\section{Reasons for participation being important}

Involvement: this refers to incidents of collaboration, or being part of the group. Behaviour: this refers to things such as showing the teacher that they are on-task and listening. 
Learning: the child refers to enhancing his or her own understandings, getting new ideas or being able to ask questions or seek clarity.

Reputation: this refers to participation having a positive impact on the child's reputation, such as other children knowing they are clever or have good ideas and getting praise from the teacher.

Reward: the child refers to the possibility for physical rewards such as stickers or prizes.

\section{Reasons for participation being not important}

Dislike: the child indicates that they do not like participating but does not necessarily give another detailed reason.

Performance: this refers to anxiety or concern in that the child might be worried about saying or doing the wrong thing, or getting answers wrong. Shyness: this is more relational. The child refers directly to shyness or uncertainty about the group or people in the group.

\section{Enjoyment of mat time}

1. 'Happiness' and 'enjoyment' are treated as synonymous. Similarly, 'dislike' is treated as synonymous with 'unhappy'.

2. Some children nominate more than one category (e.g. in between happy or neutral, or they nominate one aspect that makes them happy and one that makes them unhappy). In each case, please read subsequent comments and decide which category they most likely fit into.

3. Some children nominate one category but give contradictory reasons (e.g. they might say that mat time makes them happy, but when asked what they like about it, say they like it when mat time is finished). In such cases, code in accordance with their qualifying statements, rather than their nomination of feeling. In the example given, it would be coded as "not enjoyed".

4. Definitions are as follows:

Lack of enjoyment: the child nominates the unhappy face and/or makes statements that indicate they dislike mat time.

Neutral: The child nominates the neutral face.

Enjoyment: the child nominates the happy face and/or makes positive qualifying statements about things that they like.

\section{Things that children like/enjoy about mat time}

Children may indicate more than one category. Please count all of the categories that each child refers to.

Activity: this includes activities such as games, books, massage, technology, etc. Learning: the child might not refer to a specific activity but refers to learning something new, hearing other people's ideas or finding answers to questions. 
Togetherness: the child refers to positive aspects of the entire group being together, e.g. they like being with everyone. It also includes finding out more about other peers or working alongside children they do not know as well. Rewards: Physical rewards such as stamps or prizes.

Reputational factors: This includes academic reputation such as showing that they are clever or knowledgeable. This also includes behavioural reputation such as not getting into trouble, being noticed for being good.

Friendship: sitting next to friends, making new friends, building relationships. Seating: they like it when they get a "good" seat, or refer to getting a particular position.

\section{Things that children do not like/enjoy about mat time}

Activity: they nominate mat time activities that they do not like or they say that they find it boring.

Disruptions: this includes disruptive behaviours from other children, noise levels, finding it hard to focus.

Reputational factors: getting answers wrong or getting in trouble.

Physical discomfort: they find sitting on the mat physically uncomfortable, they feel squashed, parts of their body get sore at times.

Length: they find mat time too long.

Friendship: they refer to friendship issues or not being chosen.

Seating: they cannot get the seat that they want, they cannot see or hear where they usually sit. 


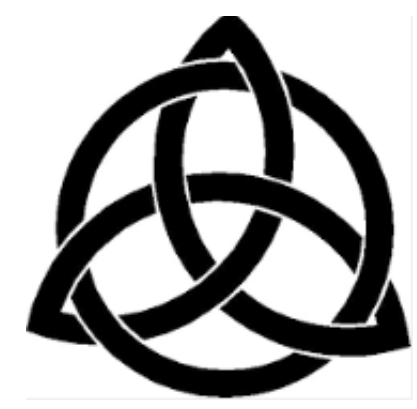

268 